\title{
On the assessment of symptom validity in refugee mental health
}

Citation for published version (APA):

van der Heide, D. H. (2021). On the assessment of symptom validity in refugee mental health. [Doctoral Thesis, Maastricht University]. Maastricht University. https://doi.org/10.26481/dis.20210527dh

Document status and date:

Published: 01/01/2021

DOI:

$10.26481 /$ dis.20210527dh

Document Version:

Publisher's PDF, also known as Version of record

\section{Please check the document version of this publication:}

- A submitted manuscript is the version of the article upon submission and before peer-review. There can be important differences between the submitted version and the official published version of record.

People interested in the research are advised to contact the author for the final version of the publication, or visit the DOI to the publisher's website.

- The final author version and the galley proof are versions of the publication after peer review.

- The final published version features the final layout of the paper including the volume, issue and page numbers.

Link to publication

\footnotetext{
General rights rights.

- You may freely distribute the URL identifying the publication in the public portal. please follow below link for the End User Agreement:

www.umlib.nl/taverne-license

Take down policy

If you believe that this document breaches copyright please contact us at:

repository@maastrichtuniversity.nl

providing details and we will investigate your claim.
}

Copyright and moral rights for the publications made accessible in the public portal are retained by the authors and/or other copyright owners and it is a condition of accessing publications that users recognise and abide by the legal requirements associated with these

- Users may download and print one copy of any publication from the public portal for the purpose of private study or research.

- You may not further distribute the material or use it for any profit-making activity or commercial gain

If the publication is distributed under the terms of Article $25 \mathrm{fa}$ of the Dutch Copyright Act, indicated by the "Taverne" license above, 
ON THE

\section{ASSESSMENT OF SYMPTOM VALIDITY \\ IN REFUGEE MENTAL HEALTH}

Douwe H. van der Heide 
(C) Douwe H. van der Heide, Maastricht, the Netherlands, 2021.

All rights reserved. No part of this book may be reproduced, stored in a retrieval system of any nature, or transmitted in any form or by any means (electronic, mechanical, photocopying, recording or otherwise) without prior written permission of the author, or when appropriate, by the publishers of the publications.

ISBN: 978-94-6421-321-8

Cover art: Foto Harderwijk B.V.

Lay out: Wendy Bour-van Telgen

Production: Ipskamp Printing, Enschede 


\title{
ON THE ASSESSMENT OF SYMPTOM VALIDITY IN REFUGEE MENTAL HEALTH
}

\author{
PROEFSCHRIFT
}

Ter verkrijging van de graad van doctor aan de Universiteit Maastricht, op gezag van de Rector Magnificus, Prof. dr. Rianne M. Letschert, volgens het besluit van het College van Decanen,

in het openbaar te verdedigen op donderdag 27 mei 2021 om 10:00 uur

door

Douwe Hendrik van der Heide

Geboren 1 mei 1961 te Ferwerderadeel 


\section{Promotores}

Prof. dr. H.L.G.J. Merckelbach

Prof. dr. P.N. van Harten

\section{Beoordelingscommissie}

Prof. dr. R.W.H.M. Ponds (voorzitter)

Prof. dr. J.M.L.G. à Campo

Dr. B. Dandachi-FitzGerald

Prof. dr. mr. M.J.J. Kunst (Universiteit van Leiden)

Prof. dr. B.A. Schmand (Universiteit van Amsterdam) 
In memory of Attila Kövi (1924 - 2004)

Refugee; teacher. 


\section{TABLE OF CONTENTS}

List of abbreviations

CHAPTER 1 General introduction

CHAPTER 2 Overlooking feigning behavior may result in potential harmful treatment interventions: Two case reports of undetected malingering

CHAPTER 3 Tranylcypromine and khat: A potentially fatal combination

CHAPTER 4 Validity of symptom reports of asylum seekers in a psychiatric hospital: A descriptive study

CHAPTER 5 The mirror sign: A serious symptom? Not really...

CHAPTER 6 Standard symptom inventories for asylum seekers in a psychiatric 73 hospital: Limited utility due to poor symptom validity

CHAPTER 7 Psychosis as a confounder of symptom credibility testing in a 93 transcultural sample

CHAPTER 8 The floor effect strategy for the assessment of performance validity in 107 refugee mental health patients

CHAPTER 9 Does geography confound performance validity test outcomes? 
Summary

163

Samenvatting

171

Impact

181

References

185

Acknowledgements

209

Dankwoord

213

Curriculum Vitae

217

List of publications

221 



\section{List of abbreviations}

CCMO Centrale Commissie Mensgebonden Onderzoek

DES Dissociative Experiences Scale

DSM Diagnostic and Statistical Manual (of the American Psychiatric Association)

GGZ Geestelijke Gezondheidzorg (Mental Healthcare)

HTQ Harvard Trauma Questionnaire

MAO Monoamine Oxidase

MENT Morel Emotional Numbing Test

M-FAST Miller-Forensic Assessment of Symptoms Test

NGO Non-governmental Organization

PVT Performance Validity Test

PTSD Posttraumatic Stress Disorder

RVCS Reversible Vasoconstriction Syndrome

SIMS Structured Inventory of Malingered Symptomatology

SIRS Structured Inventory of Reported Symptoms

SPST Study Path Selection Test (Trajectkeuze Toets)

SRVT Self-Report Validity Test

SVT Symptom Validity Test

TOMM Test of Memory Malingering

TCA Tricyclic Antidepressants 


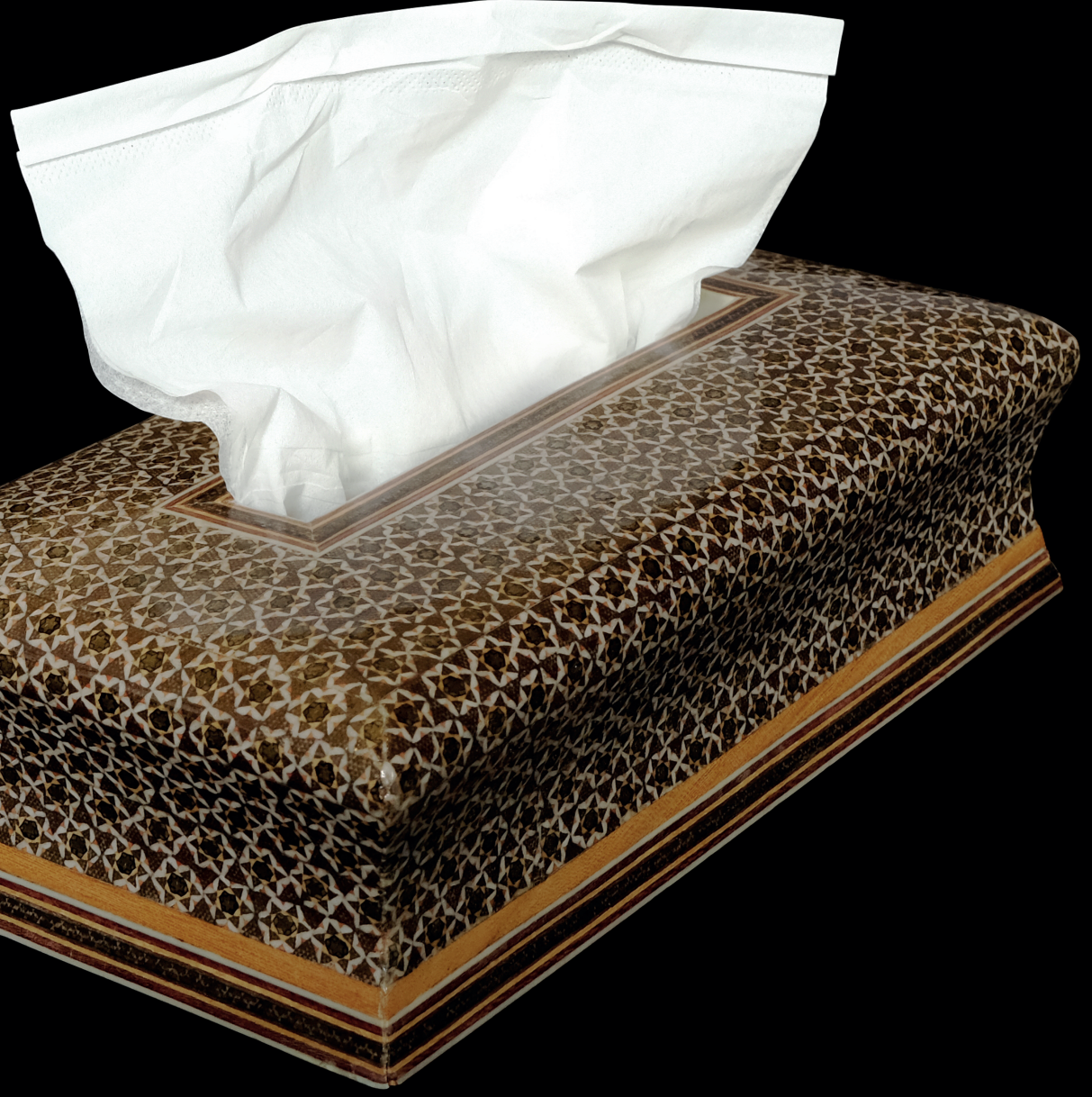


GENERAL INTRODUCTION 


\section{If in doubt, abstain?}

In the judicial system, the conviction of an innocent citizen is an error far more serious than the acquittal of a guilty person. Conversely, in medical procedures unnecessary treatment of a healthy person is more acceptable than mistakenly withholding treatment from a genuinely ill patient (Rassin \& Merckelbach, 1999). Nevertheless, unnecessary treatment should be avoided as well, especially if harmful side-effects are to be expected. Thus, in keeping with the doctrine of "primum non nocere" (first do no harm), before starting treatment, expected beneficial effects are weighed against possible negative outcomes. Only if the stakes are high, i.e., if the consequences of no treatment are particularly detrimental, serious side-effects become acceptable. On the other hand, if this is not certain, for example because there is no firm diagnosis, a prudent decision may be to defer treatment: "in dubio, abstine" (if in doubt, abstain). For mental health professionals, this last point is especially relevant, as the diagnostic findings in this field usually do not include "hard data" such as lab or scan results. The case vignette that follows is about a psychiatric patient whose diagnosis was uncertain, while treatment had no effect other than side-effects. Yet, the (social) consequences of withholding treatment were likely to be detrimental. Should the "in dubio, abstine" principle prevail, or not?

\section{Case 1}

A. was a 34-year old illegal immigrant who was referred for admission into a clinical center for refugee mental health in the Netherlands. The referral was requested by a forensic psychologist in the detention center where $\mathrm{A}$. had been held pending extradition, after his arrest at a local building site during a routine check for illegal workers. A. had told the psychologist that he was forced to commit suicide by evil spirits in the detention center who attempted to rape him at night. Once admitted to the refugee mental health center, he revealed that he had been molested in his home country because of his homosexual orientation; out of shame, he had failed to mention this during a previous procedure for asylum. Even now A. insisted on confidentiality as he was a pious member of an evangelical cult, participating in "prayermarathons" in his church during weekend furloughs. He absconded from treatment after the police made another, unsuccessful attempt to arrest him. Apparently, this time he was suspected of armed robbery. The police had been tracking his mobile phone; during international phone calls from a location inside the refugee mental health center, they overheard him discussing his participation in several robberies during weekends.

A few days after his disappearance, a Dutch woman called the referral center, introducing 
herself as A.'s girlfriend for the past few years. She was worried as he had failed to contact her since he absconded. Out of this contact it eventually emerged that, as his girlfriend, the woman was having a mutually satisfactory sexual relationship with A.; also, she had not been aware of any of his psychological problems until his admission.

A few weeks later the staff of the center learned that A. had been arrested after all and transferred to the psychiatric ward of the prison where he was awaiting trial. A. had alerted a forensic psychiatrist to his psychological problems and requested that he contacted the refugee mental health center. During this consultation, the staff discussed their current doubts regarding A.'s psychiatric diagnosis; nevertheless, the forensic psychiatrist decided that he most likely suffered from an acute psychotic breakdown related to previous traumatic experiences, and that he needed treatment with antipsychotic medication.

A few months later, the staff of the center was contacted yet again, by another psychiatrist, also after a request by $\mathrm{A}$. He had been released from prison and referred to an outpatient mental health team. Despite treatment with long-acting antipsychotic medication, with considerable side-effects, his symptoms were therapy-resistant; the psychiatrists suspected that this was due to cultural factors and asked for consultation by a refugee mental health specialist. During this consultation, the clinic staff discussed their doubts about the diagnosis as well. Once again, the psychiatrist decided to continue antipsychotic treatment.

After this, the patient was lost to follow-up. Most likely, his antipsychotic treatment was continued for at least some time; two psychiatrists already decided to either initiate or continue antipsychotic treatment, despite serious doubts about the psychiatric diagnosis, lack of therapeutic effect, and considerable side-effects. This course of events started a discussion among the staff of the refugee mental health care center. In the end, most of their arguments were in support of the decision to ignore the "in dubio, abstine"- principle in this case:

1. "Even if A. feigned symptoms, that in itself does not exclude a mental disorder." Staff members argued that the likelihood of severe psychological trauma in refugee mental health patients is considerable, and that the risk of withholding treatment to a genuinely ill patient should be avoided at all cost.

2. "Even if A. lied about his traumatic experiences, that does not mean that he did experience no traumatic events at all." Accurate and reliable information about traumatic events is important for treatment. However, in refugee mental health such information may also serve to support a claim for asylum. In the months preceding A.'s admission, there had been several news items in the Dutch media about discrimination and persecution of gay 
men in Sub-Saharan Africa, followed by protests by human rights groups. Did A. lie about his sexual orientation for the sake of a new procedure for asylum, and hence also about the abuse he had suffered? Staff members argued that even if he did so, he might still have suffered other forms of abuse. Besides, they argued, the information provided by his girlfriend proves nothing; in homophobic societies, people with a homosexual orientation may become well accustomed to leading a double life.

3. "Diagnostic uncertainty is inherent to mental disorders." Even in the face of collateral information, the variability in course and presentation of these disorders allows well for striking inconsistencies. For instance, staff members argued that the absence of symptoms before A.'s detention might be explained as a symptom free interval that is typical of the late-onset variety of post-traumatic stress disorder (PTSD), with A.'s arrest and detention as the final trigger to the onset of symptoms. Some argued that even his criminal activities might be interpreted as a way of coping with psychological trauma - "turning passive into active", or "identification with the aggressor". Finally, according to some staff members, the absence of therapeutic effect should be taken to support the conclusion that his psychotic-like symptoms were, in fact, related to trauma.

4. "If the consequences of no treatment are particularly detrimental, the "in dubio, abstine" principle becomes less relevant." The stakes were high in A.'s case; without a psychiatric diagnosis and its subsequent need for treatment, he might well have been extradited, or at least be forced back into the life of a marginalized illegal immigrant. According to these professionals, that should be enough reason to overrule the fact that a diagnosis of psychotic disorder in this case was uncertain, even more so because to them it was not evident whether A. had been feigning or not, and even if he had, to what extent.

\section{How to substantiate clinical suspicion of feigning}

Apparently, evidence for feigning must be substantial before it becomes relevant in high stake treatment decisions in refugee mental health, with protracted admissions and treatments with possible harmful side-effects that may not be necessary at all as a result. So, how is evidence of feigning substantiated in other fields of mental health? Before we proceed to the multimethod approach that is advocated for this purpose in forensic psychology, we should define "feigning".

Feigning is the deliberate fabrication or gross exaggeration of symptoms, regardless of possible motives or incentives (American Psychiatric Association [APA], 2013). This definition 
rules out conversion disorder; symptoms of this disorder cannot be attributed to a physical cause, but they are taken not to be the result of intentional production either (Kanaan, 2018). Within DSM-5, there are two diagnostic classifications associated with feigning: factitious disorder and malingering (APA, 2013). The two are distinguished by the type of motive or incentive that causes feigning behavior. In factitious presentations, symptoms are feigned in the absence of obvious external rewards. Possibly, the motive is internal, e.g., assuming the "sick role", so as to obtain attention from others, primarily medical professionals. In contrast, a malingerer feigns exclusively to obtain an external incentive, e.g. a refugee status, or medical asylum (Rogers, 2018).

Persistent feigning of serious, possibly even life-threatening symptoms is usually only observed in factitious disorders; malingerers tend to make a more rational stance towards the risks they are willing to take and tend to be "cured" shortly after the external reward is obtained (Feldman, 2004). However, malingerers may underestimate the risks of unnecessary treatment, or the length of time necessary to obtain their goal. They may not be aware that both psychiatric treatment and protracted admission can have substantial negative effects, also in healthy people. A prolonged period of admission induces demoralization and dependency and eventually promotes behavioral disturbances, especially so in the malingerer who may feel that keeping up a dramatic symptom presentation is necessary to remain convincing (Frueh et al., 2005). Psychiatrists will prescribe medication with few side-effects at the start of treatment, but if symptoms persist more hazardous drugs tend to take their place (APA, 2010). And even if this does not occur, common side-effects of psychiatric medication (e.g., metabolic side-effects; Tschoner et al., 2007) may also decrease the quality of life and life-expectancy considerably. The longer the period that a malingerer needs to maintain the feigned symptom presentation, the higher the risk of iatrogenic damage becomes. For refugee mental health patients in the Netherlands, this is particularly relevant: procedures for asylum in this country may take many years (Rijksoverheid, 2019).

Collateral information, as in A.'s case, does not suffice to establish whether a patient is feigning, although it does constitute an important part of the multimethod approach that is used to examine suspected feigning. In neuropsychology, clinicians often adopt Slick et al.'s criteria to determine whether a patient does engage in feigning behavior (Sherman et al., 2020; but also see: Slick et al., 1999; Slick \& Sherman, 2012; Young, 2014). The Slick criteria involve the presence of a substantial external incentive (criterion A); underperformance on psychometric tasks and/or symptom over-reporting on psychometric tests (criterion B); self- 
reports that are discrepant with collateral information, and/or self-reports that are discrepant with observation (criterion C); and furthermore, behaviors meeting B or C should not be fully accounted for by psychiatric, neurological or developmental factors.

To screen for exaggerated or feigned symptom presentations (criterion B) increasingly effective Symptom Validity Tests (SVTs) have been developed during the past decades (Bush et al., 2005; Heilbronner et al., 2009; Merten \& Merckelbach, 2013). Most SVTs are inventories of unlikely or non-existent symptoms and are based on the rationale that patients who exaggerate symptoms are likely to endorse rare or unlikely items as well. This type of SVT is also known as Self Report Validity Test (SRVT). Examples are the Miller-Forensic Assessment of Symptoms Test (M-FAST; Miller, 2001), the Structured Inventory of Malingered Symptomatology (SIMS; Widows \& Smith, 2005), and the Structured Interview of Reported Symptoms (SIRS; Rogers et al., 2010).

\section{Symptom validity assessment in refugee mental health}

In refugee mental health, however, the use of these instruments is regarded as precarious (Nijdam-Jones \& Rosenfeld, 2017). This is because in SVTs, a negative outcome (i.e., passing the SVT) is generally more informative than a positive one (i.e., failing the SVT). A negative outcome effectively decreases the likelihood that the patient is malingering, whereas a positive outcome merely indicates poor symptom validity, irrespective of its cause (Merten \& Merckelbach, 2013; Young, 2014). In fact, malingering is only one of the causes of poor symptom validity. Other possible factors are severe mental disorganization in psychotic patients (Dandachi-FitzGerald et al., 2011), intellectual disability (Shandera et al., 2016; see also Victor et al., 2009), and careless responding in general (Lippa, 2017; Meyer et al. 2013). In refugee mental health, there are additional confounding factors. For instance, as most SVTs are developed in high-income countries, they are, in view of the differences in language and culture worldwide, not necessarily suitable for migrants from low- or middle-income countries (Weiss \& Rosenfeld, 2017). Some translated SVTs were found to function reasonably well in other populations (Nijdam-Jones \& Rosenfeld, 2017), but for populations as diverse as refugee mental health patients properly translated and validated instruments are usually not available. In that case, an option is to have psychological test items translated on the spot by dialogue interpreters. This practice, however, may also jeopardize the accuracy of the test (Bot, 2005; Correa, 2018).

One possible way to circumvent this problem might be to resort to Performance Validity 
Tests (PVTs), another type of SVT, which also gauge Slick et al.'s B criterion. PVTs are based on the premise that patients who engage in symptom exaggeration will fail on cognitive tests with a very modest cognitive load (Lippa, 2017). Good examples are provided by Morel's Emotional Numbing Test (MENT; Morel, 1998), which is a simple forced-choice task developed to screen for exaggeration of trauma-related impairment, or Tombaugh's Test of Memory Malingering (TOMM; Tombaugh, 1996), which screens for the exaggeration of memory impairment. Importantly, tests such as the MENT and the TOMM have minimal verbal mediation, which reduces the risk that differences in language confound the test outcomes (Erdodi et al., 2017). Still, verbal mediation is only one of several factors affecting test outcomes in intercultural settings. Other factors, such as cultural differences in test taking attitude may also be important as confounder (Ardila, 2005).

In light of these considerations, SVTs are currently not used to check the validity of self-reported symptoms in refugee mental health. This has a variety of consequences. For instance, for epidemiological research, where the accuracy of prevalence estimates of various disorders is at stake. Many papers have inferred raised levels of psychopathology in refugee mental health patients from their self-reports, but as long as the validity of these reports is uncertain there is reason to doubt the accuracy of these findings (Rosen, 2004, 2006; Rosen \& Taylor 2007). For clinical practice, a consequence may be that scarce resources needed to treat psychiatrically ill patients in refugee mental health are used for secondary purposes instead. More hazardous, refugee mental health patients may be exposed to potentially serious side-effects of treatments that might not have been indicated after all.

\section{Aims and outline of this thesis}

Given that diagnosis and treatment rest on an accurate assessment of symptoms, there is a need for exploring symptom validity in the clinical practice of refugee mental health. That is precisely what the chapters in the current thesis do. More specifically, this thesis will address the following research questions:

1. Is clinical observation, even when it is done during a protracted period of admission in a specialized center, sufficient to rule out illness distortion? (Chapter 2).

2. Are there special risks when clinicians rely on their clinical impression in the case of patients with a different cultural background? (Chapter 3);

3. Are severe dissociative symptoms reported by refugee mental health patients associated 
with higher scores on SVTs? (Chapter 4);

4. Is the "mirror sign" an early psychotic symptom? (Chapter 5);

5. To what extent do deviant SVT scores of refugee mental health patients reflect their poor skills in the language of the host country rather than feigned symptoms? (Chapters 4, 6 and 9);

6. In refugee mental health patients, is deviant performance on SVTs associated with escalated symptom endorsement on standard diagnostic instruments? (Chapter 6);

7. Does a psychotic disorder confound the outcomes of SVTs in refugee mental health patients? (Chapter 7);

8. Do regional differences in the world confound the outcomes of SVTs in refugee mental health patients? (Chapter 8 and 9).

The main results, as well as methodological and clinical considerations, and directions for future research, are discussed in Chapter 10.

\section{A short note on DSM's criminological typology of feigning and malingering}

Many researchers (e.g., Berry \& Nelson, 2010; Niesten et al., 2015) have taken issue with the way in which the Diagnostic and Statistical Manual of the American Psychiatric Association (DSM) portrays feigning and, particularly, malingering. In short, the successive editions of the DSM suggest that symptom over-reporting is what people with antisocial personality traits do when they are faced with legal problems. Thus, there are strong moral overtones in DSM conceptualization of malingering. That is not the stance taken in this thesis. Here, we follow Rogers (1990) adaptational view on symptom over-reporting, exaggeration, and malingering: it is what reasonable people do when they are confronted with harsh and adversarial circumstances and there are no alternatives. In many ways, the position of refugees - with their problematic backgrounds, the extensive procedures they are involved in, the unfamiliar host country that they find themselves in - may fall under the definition of adversarial circumstances. In that context, recognizing symptom over-reporting and exaggeration may be a first step in understanding the real problems of the patient. 



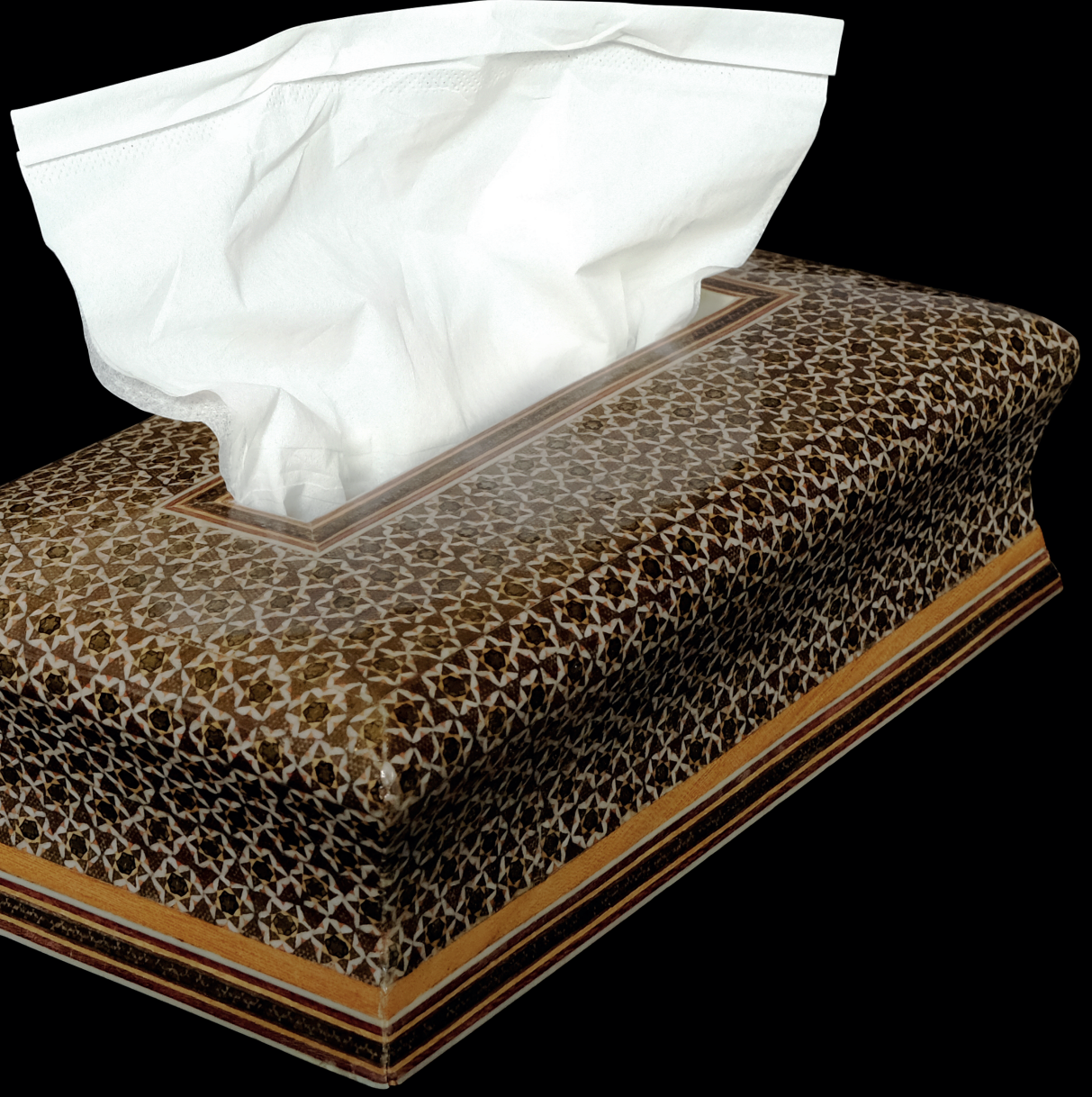




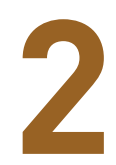

\section{OVERLOOKING FEIGNING BEHAVIOR MAY RESULT IN POTENTIAL HARMFUL TREATMENT INTERVENTIONS: TWO CASE REPORTS OF UNDETECTED MALINGERING}

Published as:

Van der Heide, D., Boskovic, I., Van Harten, P., \& Merckelbach, H. (2020). Overlooking feigning behavior may result in potential harmful treatment interventions: Two case reports of undetected malingering. Journal of Forensic Sciences, 65(4), 1371-1375.

https://doi.org/10.1111/1556-4029.14320 


\section{Abstract}

Clinicians tend to overestimate their ability to recognize feigning behavior in psychiatric patients, especially if it concerns patients who have been admitted for observation. Feigning can be either externally motivated (e.g. for financial compensation, known as malingering) or internally motivated (e.g. to assume the "sick role", known as factitious disorder). Persistent presentation of severe symptoms is usually associated with the factitious disorder. We present two patients with strong external incentives who consistently and convincingly feigned severe psychiatric symptoms during a protracted period of inpatient observation in a specialized center; both were engaged in a procedure for medical asylum. The first case presented with the clinical picture of a psychotic depression with severe motor symptoms and the second case showed symptoms of a chronic post-traumatic stress disorder with secondary psychotic symptoms. Both cases were thoroughly investigated but feigning was overlooked, and unnecessary and harmful treatment interventions were given. To prevent iatrogenic damage, we recommend a critical attitude that takes feigning as an option into account in settings were patients are often involved in high stake legal procedures. A clinical sign that might indicate feigning are therapyresistant symptoms. To rule out feigning a comprehensive, multimethod approach is required, but an active stance towards collateral information is essential. Specialized psychological tests may be useful for preliminary screening, but for their use in culturally diverse populations as in refugee mental health more research is needed. 


\section{Introduction}

Despite advances made in the diagnosis and treatment of psychiatric disorders, considerable numbers of psychiatric patients suffer from symptoms that remain resistant to treatment (Smith-Apeldoorn et al., 2019). Patients in refugee mental health settings are no exception; psychiatric symptoms are prevalent in this group (Richter et al., 2018) and their symptom severity tends to increase when they become involved in legal procedures for asylum (Laban et al., 2004). Understandably, mental health professionals are concerned about the accessibility and quality of psychiatric care for this vulnerable group (World Psychiatric Association [WPA], 2017). With this in mind, specialized facilities for refugee mental health have been created in the Netherlands to which patients with therapy-resistant symptoms can be referred. The referral center described below had 32 beds and a staff consisting of two psychiatrists, three psychologists, two social workers, and two Dutch language teachers. Additionally, it included facilities for art, music, and occupational therapy. Although the majority of patients referred to such centers report traumatic events, most of them meet not only diagnostic criteria for PTSD (APA, 2013), but may also suffer from other disorders, such as a psychotic disorder or an affective disorder. In a non-trivial minority, there is a comorbid personality disorder and/or a co-morbid substance use disorder. Despite treatment in these specialized centers, some patients remain substantially impaired by their symptoms. As the procedure for asylum usually requires several transfers to other regions of the country, reallocation to regular care after treatment is often a time-consuming process, resulting in an average duration of an admission varying between 6 and 9 months.

In the two cases described below, a radical revision of the initial diagnostic classification was necessary due to clinicians overlooking feigning. Feigning is the deliberate fabrication or gross exaggeration of symptoms, regardless of possible motives. It is important to note that this definition rules out conversion disorder; although a physical cause is absent in conversion symptoms, they are not assumed to result from intentional production (Kanaan, 2018). Clinical entities that are associated with feigning are the factitious disorder and malingering. In factitious presentations symptoms are feigned in the absence of obvious external rewards, but possibly out of some internal motivation, e.g. to assume the "sick role". Although patients can be both internally and externally motivated at the same time, a malingerer is exclusively motivated by an external incentive (American Psychiatric Association, 2013; Rogers, 2018). Cases with consistent feigning of extremely serious symptoms are usually observed in factitious disorders 
(Feldman, 2004). In the cases described here, both about a person seeking asylum, there was a potentially strong external incentive; both patients were involved in a procedure for medical asylum. In procedures for asylum, symptoms compatible with trauma are sometimes used to support the credibility of reports about human rights abuse and in case of a medical disorder, including mental disorders, a refugee status may be granted for humanitarian reasons, even if a previous request for political asylum was denied (Wijnkoop, 2006).

\section{Case 1}

A 56-year-old man seeking asylum in the Netherlands was admitted because of mutism and severe psychomotor retardation. According to his relatives, he already had symptoms in his country of origin. They reported that his sleep was frequently interrupted by nightmares and anxiety up to the point that he felt so tired that he hardly left his house anymore; apparently, the symptoms had started after a period of detention and torture. He also lost interest in his wife and children although, according to his relatives, he used to be a "real family man". After his arrival in the host country, he stayed in one of the centers where individuals seeking asylum in the Netherlands are housed pending their procedure. Here, his condition appeared to deteriorate following a negative decision on his application for asylum. He lost interest in life altogether and became apathetic until he was bed-ridden and no longer able to move or speak, having to be hand-fed by his relatives. At the time of his referral a procedure for medical asylum had been started. On admission to the referral center, we saw a disheveled-looking man with long hair and an unkempt beard who did not react to any attempt to start a conversation. His face was frozen into an expression of fear and disgust and only after encouragements by his wife, he was able to give a minimal non-verbal reaction. Examining his motor symptoms, we found active muscular resistance but no signs of passive rigidity such as extrapyramidal rigidity or waxy flexibility. There was a minimal contracture in the shoulder region, but no bedsores or signs indicating vegetative dysregulation. Later on, during his stay in the referral center, he appeared to be distracted by objects invisible to others.

On the basis of extensive laboratory analyses and neuroimaging of the brain, the referring psychiatrist had ruled out any somatic causes of his condition and had started treatment with serotonin re-uptake inhibitors (SSRI) and later tricyclic antidepressants (TCA), without this having any therapeutic effect. In the referral center, benzodiazepines, lithium, and later antipsychotics were added to this regimen, under the provisional diagnosis of a psychotic depression with catatonic features. 
Despite continuous attempts to activate the patient, he made no progress except that after a few months of admission, he was able to eat a few spoonsful of porridge by himself. Given these poor results, his medication was replaced by tranylcypromine and preparations were made to refer him for electroconvulsive treatment as a final attempt to improve his condition.

At this point, a staff member of the referral center happened to observe the patient, during a weekend outside the hospital. At this occasion, the patient was engaged in a lively conversation while having lunch with his family. Although his relatives denied any improvement of this sort, the observation was consistent with those of staff members of the asylum seeker center where the patient had initially lived. They had spotted the patient at night while moving around actively in his quarters, eating and drinking normally, but had failed to share this information with the staff of the referral center until they were specifically asked for it. After confrontation with this information, the patient immediately left the hospital and was lost for follow-up. In total, he had stayed in the referral center for four months.

\section{Case 2}

An unaccompanied female minor seeking asylum in the Netherlands was admitted because of disruptive behavior in the asylum seeker center. She was initially an illegal immigrant. However, an asylum procedure had been started on her behalf after she allegedly escaped from captivity and abuse by fellow-countrymen in the host country. At the time of her referral she was in procedure for medical asylum. In the ward, we saw a thin, soft-spoken, well-dressed girl presenting herself in a child-like manner but without evident psychiatric symptoms at that moment. During the ensuing admission, she generally behaved meek and submissive, but could suddenly become confused and agitated, inflict wounds to herself and damage hospital property, violently resisting attempts to control her behavior and apparently without any recollection of the incidents afterwards.

During individual therapeutic sessions, assisted by a professional interpreter, she gradually revealed a string of dramatic events in her past that formed a pattern of severe and recurrent trauma. For example, she recalled witnessing the brutal murder of her parents as a young child and being raised by an older sister. Destitute, the girls survived by scavenging the dumping grounds of a large city. After the sister had been murdered as well, an elderly priest took her under his care, provided her with some money, and urged her to leave the country.

The therapist of the referral center was under the impression that a relationship of mutual trust was developing. Meanwhile, the symptoms of the patient deteriorated. 
She became agitated more often and started to show a preoccupation with religion and promiscuous behavior at the same time, absconding a few times in the company of unknown men. Under the provisional diagnosis of complex, early trauma with secondary psychotic features, antidepressants and atypical antipsychotics were added to her treatment. After this, her symptoms gradually improved, but she also started to gain weight at an alarming pace; by the time her disruptive behavior was mostly under control, she had become overweight, apathetic, and mostly dependent on others for her daily activities. She managed only short periods of furlough in the asylum seeker center on her own.

At this point, her legal representative reported that fellow countrymen were visiting her regularly during these furloughs, notably one elderly man. Suspicious of renewed sexual abuse, this man was confronted, upon which he identified himself as a close friend of the parents of the patient; he was even willing to share their telephone number. The collateral information provided by parents and a close friend converged to the conclusion that the girl was, in fact, a 24-year-old woman who used to be a skilled bank employee in her country of origin. She had been converted by a Western evangelical movement and travelled to the Netherlands by airplane to visit a religious conference. Soon after her arrival, she lost her documents. Being a friend of her parents, the elderly man had welcomed her into his family, where she stayed until she was taken into custody by the police one day and reported her escape from fellow countrymen. The parents were from a middle-class family and had been in contact with their daughter all the time. Her sisters were all in good health. The family was very worried, but also grateful for her treatment.

The patient left the hospital after an admission of eight months and managed to live independently with the help of members of her religious community from her country of origin.

\section{Clinical observation as a diagnostic tool}

In medicine, the error of treating a healthy person is preferred over the risk that treatment is denied to a truly ill patient (Rassin \& Merckelbach, 1999). This is particularly the case in refugee mental health, where the conclusion that a person seeking asylum has no psychiatric disorder may in some cases result in the extradition of that individual (European Council of Refugees and Exiles [ECRE], 2012). It is therefore understandable that professionals in such settings want to err on the safe side and are hesitant to question the veracity of symptoms and impairments presented by patients. In addition, mental health professionals tend to rely on clinical observation, but even clinically experienced judges are not very successful in 
differentiation between genuine and feigned symptoms (Rosen \& Phillips, 2004).

The case vignettes presented above illustrate two points. First, even though admission and clinical observation are sometimes recommended in cases where feigning is suspected (Conroy \& Kwartner, 2006), prolonged inpatient treatment and observation in a specialized psychiatric clinic may not suffice to rule feigning in or out. Second, and most importantly, patients who exaggerate or feign symptoms are, apart from the possible benefits they derive from their behavior, at risk of unnecessary treatment and even iatrogenic damage. There is evidence that both protracted psychiatric admission and treatment may have negative outcomes in healthy people. A prolonged period of admission may induce demoralization and dependency and in the long run, may even promote behavioral disturbances rather than cure them (Frueh et al., 2006). Although psychiatrists will prescribe types of medication with relative few side-effects at the start of treatment, these are likely to be replaced by more hazardous drugs if symptom reports persist (APA, 2010), as is often the case in patients who exaggerate or feign their symptoms. Even common side-effects of psychiatric medication, such as weight gain, may eventually lead to complications, such as glucose-intolerance, hypercholesterolemia, and hypertension, which decrease life-expectancy considerably (Correll et al., 2015).

Why is it that 24/7 clinical observation does not necessarily lead to straightforward conclusions when it comes to the detection of feigning? Genuine psychiatric disorders display a large diversity in course and presentation. For example, the absence of a classic sign such as waxy flexibility in the first case does not exclude a catatonic state. Further, catatonic stupor and mutism may well be interrupted by short periods of activation. Short intervals of relative competency may follow in patients who react to treatment with benzodiazepines (Bostwick \& Chozinski, 2002) and sudden bursts of activity and agitation are inherent to catatonia itself, although these features are usually erratic (De Pauw \& Szulecka, 1987). Likewise, the behavior of a traumatized patient, such as the patient described in the second vignette, may be highly variable. A meek and even submissive attitude may suddenly turn into a confused and agitated stance when triggered by events or circumstances that are reminiscent of prior trauma. Sometimes, the behavior may be counterintuitive. For example, some victims of sexual violence may show signs of promiscuity in the aftermath of their trauma (Di Giacomo \& Clerici, 2011).

Thus, the clinical pictures depicted in these vignettes were by no means exceptional for the referral center concerned. What makes these cases remarkable was that the clinical staff happened to stumble upon collateral information that was grossly incompatible with prior diagnostic conclusions. Inspection of the medical files of refugee patients referred to the 
specialized center in the 2008 - 2012 period $(N=231$ ) showed that such collateral information was collected in less than $1 \%$ of the patients admitted to the center. Thus, the uncomfortable question can be raised in how many other cases exaggerated or feigned symptom presentation might have played a role.

The outcomes in the cases described above had a large impact on the professionals involved in their treatment, with emotions ranging from frustration and regret to disbelief. Frustration and regret because of the iatrogenic damage that the patients may have sustained during their admission, and disbelief because during an admission of several months, these patients, like many other patients who were admitted, consistently presented severe signs and symptoms entirely compatible with their diagnosis and an alleged trauma history.

\section{Clinical impression and collateral information}

The self-reported trauma histories of patients who present with psychiatric symptoms may be difficult to verify and may not only serve the purpose of informing therapists but may also aim to influence legal procedures (e.g., asylum procedures, litigation claims). Further, patients who exaggerate or feign symptoms may need to maintain their symptom claims for a prolonged period of time. For example, in refugee mental health, procedures for asylum may take several years to be completed (Rijksoverheid, 2019). In this setting, it is conceivable that psychiatrists will eventually resort to prolonged periods of admission and treatments with severe side-effects and risks.

Clinicians may underestimate the prevalence of symptom exaggeration or feigning among psychiatric patients when these experts rely on clinical observation. That this prevalence may well be larger than is often assumed is suggested by rates of this behavior in samples that are in some respects (e.g., the presence of incentives) similar to the cases presented in our report. For example, reviewing the military personnel records of US veterans seeking treatment for combat-related PTSD, Frueh et al. (2005) found no evidence of combat exposure in $59 \%$ of the records, whereas in $7 \%$ there was not even documentation of Vietnam warzone service. Mittenberg et al. (2002) surveyed US neuropsychologists about base rates of symptom exaggeration and feigning. As a group, these experts typically rely on multiple methods (including psychometric tests) to determine the presence or absence of feigning. Their overall estimates ranged from 8\% (for non-litigating medical cases) to 30\% (for disability or workers compensation cases). Admittedly, base rate estimates of feigning tend to vary due to conceptual and definitional ambiguities (Young, 2014). Still, when these estimates are based 
on clinical impression alone, they might well be too low for certain settings (e.g., settings where patients are involved in legal procedures).

\section{A remedy?}

To assess the possibility of feigning or exaggerated symptom presentation a multimethod approach is needed. In neuropsychology, clinicians often adopt Slick et al.'s criteria to determine whether a patient engages in malingering (Slick et al., 1999; Young, 2014)). These criteria involve the presence of a substantial external incentive (criterion A); underperformance on psychometric tasks (criterion B); symptom over-reporting on psychometric tests, selfreports that are discrepant with collateral information, and/or self-reports that are discrepant with observation (criterion C); and furthermore, behaviors meeting B or C should not be fully accounted for by psychiatric, neurological or developmental factors.

As to criterion C, Symptom Validity Tests (SVTs) may be used to screen for exaggerated or feigned symptom presentations (Bush et al., 2005). SVTs usually list bizarre or non-existent symptoms and are based on the rationale that patients who exaggerate symptoms are likely to endorse rare or unlikely items as well. One example is the Structured Inventory of Malingered Symptomatology (SIMS), which is an inventory that consists of 75 rare or unlikely symptoms (Merckelbach \& Smith, 2003; Smith \& Burger, 1997). Some translated versions of SVTs appear to be functioning well in populations for whom they were not originally developed (Nijdam-Jones \& Rosenfeld, 2017), but in populations as culturally and linguistically diverse as patients in referral centers for refugee mental health, properly translated instruments are often unavailable. One possible way to circumvent this problem might be to resort to Performance Validity Tests (PVTs), which correspond to Slick et al.'s B criterion. PVTs are based on the premise that patients who engage in symptom exaggeration will fail on cognitive tests with a very modest cognitive load. A good example is provided by Morel's Emotional Numbing Test (MENT), which is a simple forced-choice task developed to screen for exaggeration of trauma-related impairment (Morel, 1998). Importantly, tests such as the MENT have minimal verbal mediation, which reduces the risks of language difficulties distorting the test outcomes (Erdodi et al., 2017). Still, verbal mediation is only one of several factors affecting test outcomes in intercultural settings. Other factors, such as culturally related differences in test taking attitude may be just as important as confounder (Ardila, 2005). Therefore, separate validation of PVTs for diverse cultural or linguistic groups is an important endeavor for future research (Correa, 2018).

The importance of the Slick et al. criteria is that they encourage clinicians who work 
in settings with raised base rates of feigning to move beyond clinical intuition and to take other sources of information into account, including psychometric and collateral data. To be sure, medical staff should not try to serve as private detectives and examining the Slick et al. criteria in each patient with external incentives and therapy-resistant symptoms is not necessary. In the first case that we described, just obtaining collateral information from staff workers in the asylum seeker center already turned out to be informative (Beach et al., 2017). In the second case, a high error score on the MENT, even though by itself no proof of feigning or exaggeration, could have alerted the clinical staff to this possibility at a much earlier stage (Van der Heide et al., 2017) and might have prevented her subsequent exposure to a protracted period of admission and the side-effects of antipsychotic medication. Thus, an open and active attitude towards collateral information may alert the clinician to the option of feigning, whereas a SVT or a PVT may serve as a preliminary screener before a decision to start more comprehensive investigations along the criteria of Slick needs to be made.

\section{Conclusion}

The take-home messages of our vignettes can be summarized as follows. First, patients may exaggerate or feign severe psychiatric symptoms consistently throughout a protracted admission in a specialized center without being detected. Second, this behavior may help patients to obtain certain external benefits, but it may also lead to unnecessary, potentially harmful treatment interventions. Third, to prevent these harmful interventions, clinicians who work in settings where patients are often involved in high stake legal procedures should be aware of feigning as a distinct possibility and be wary of their clinical judgement. Rather they should develop an attitude of respectful skepticism, which considers the option of feigning, and an active stance towards the collection of collateral information. Fourth, the detection of exaggeration or feigning requires a multimethod approach, yet such comprehensive approach is not necessary in all therapy-resistant cases with possible external incentives. High-risk patients may be screened with relatively simple psychometric measures to establish whether follow-up with a multimethod approach is indicated. No test or series of tests ever unequivocally proves feigning or the absence of feigning. For use of SVTs and PVTs in culturally diverse groups as in refugee mental health more research is needed. 



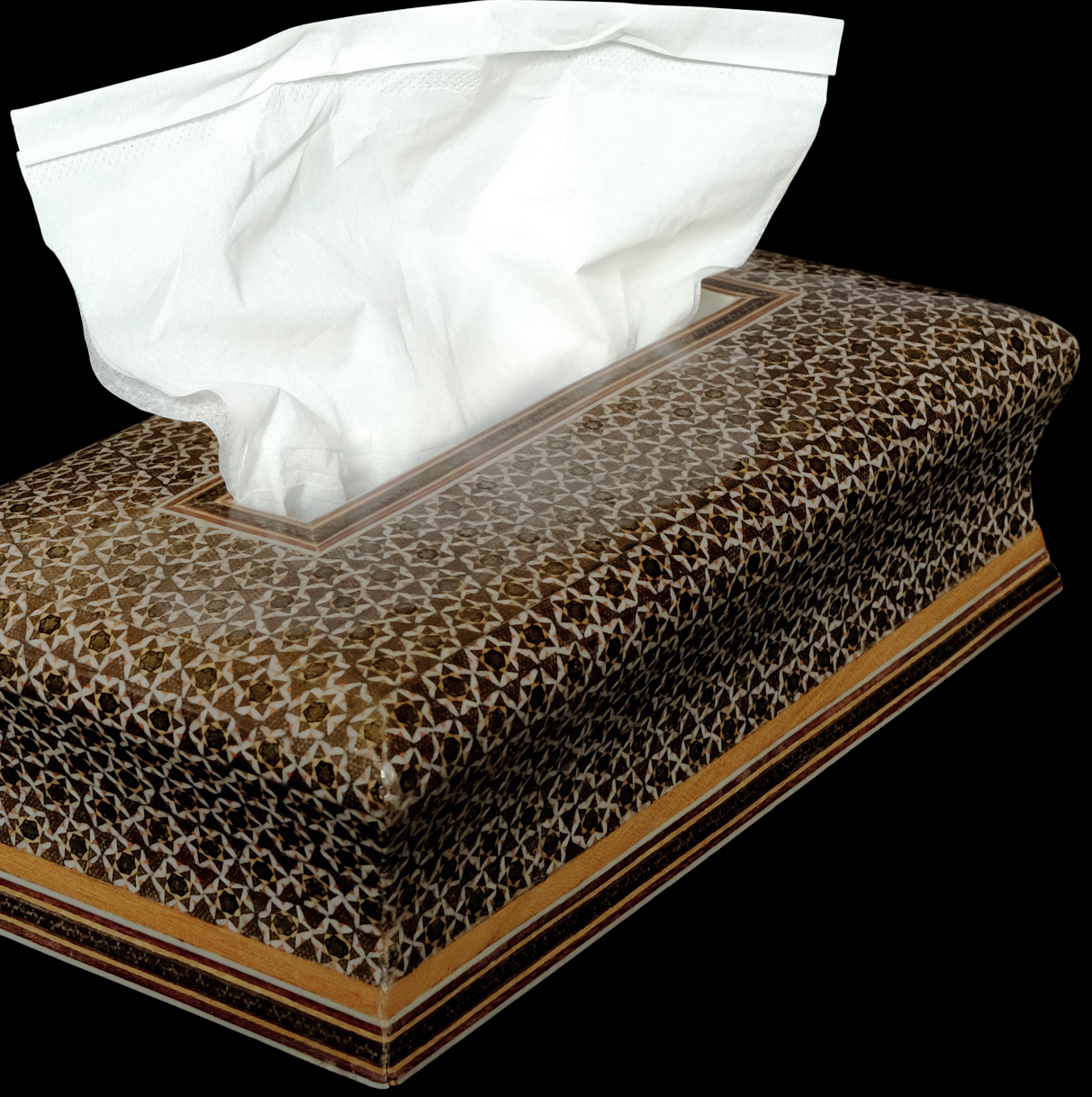




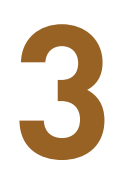

\section{TRANYLCYPROMINE AND KHAT: A POTENTIALLY FATAL COMBINATION}

\section{Published as:}

Van der Heide, D., Merckelbach, H., \& Van Harten, P. (2018). Tranylcypromine en khat: Een potentieel fatale combinatie. Tijdschrift voor Psychiatrie, 60(8), 544-547.

https://www.tijdschriftvoorpsychiatrie.nl/assets/articles/60-2018-8-artikel-vanderheide.pdf 


\section{Abstract}

The use of tranylcypromine in combination with amphetamines is known to induce a potentially lethal hypertensive crisis. However, this complication may also occur when tranylcypromine is combined with khat. Here we describe the case of a young patient who received a low dose of tranylcypromine and then used a small amount of khat. He subsequently developed a subarachnoid hemorrhage. 


\section{Introduction}

Irreversible MAO inhibitors like tranylcypromine and phenelzine are used for the treatment of depressive disorders and are included as such in the current psychiatric guidelines in the Netherlands (Spijker et al., 2013). They are indicated only for therapy-resistant cases, as they may cause severe complications, such as the "cheese reaction". This is a hypertensive crisis that occurs when patients medicated with irreversible MAO inhibitors ingest food that is rich in tyramine, such as aged cheese. Tyramine is a precursor of monoamines, and any surplus is usually eliminated by monoamine-oxidase. If this enzyme is inhibited, the compensatory mechanism fails, leading to dangerous overproduction of monoamines. Even low doses (10 mg) of tyramine may cause a severe crisis, so patients medicated with irreversible MAO inhibitors need to adhere to a tyramine-restricted diet (Flockhart, 2012). Stimulants like amphetamines may also cause hypertensive crises (Sloan et al., 1991; Gillman, 2002; Ricken et al., 2017); severe complications have also been described after a minimal dose of amphetamines in combination with tranylcypromine (Zeck, 1961; Hirsch et al., 1965; Gillman, 2002).

Due in part to the increasing cultural diversity of Dutch society, health-care practitioners are being confronted with patients who are consuming unfamiliar herbs and substances. These include medicinal herbs as well as psychoactive substances that are part of local cultures elsewhere in the world, and have found their way into Western Europe in the wake of immigration. A prominent example is khat (or qat), a stimulant that has been used on the Arabic peninsula for centuries. Its effect is obtained by chewing leaves of the khat brush (Catha Edulis). Several leaves at a time are chewed, with several portions per session. In Western Europe, khat is popular within the Somalian immigrant community. As the leaves lose their effect after 48 hours if they are not kept under refrigeration, they are imported by air (Cox \& Rampers, 2003; Wabe, 2011). Traditional substances and herbs may also cause complications when used in combination with regular medications (Rommel \& Bailey, 2006). Khat is no exception, as is illustrated by the following case report.

\section{Case report}

The patient, A., was a highly educated 30-year-old naturalized Dutch citizen with a migration background; as a teenager he left East Africa as a refugee for Europe. In recent years he had been treated with tranylcypromine for therapy-resistant depression, which had been tapered down to a daily dose of ten milligrams; the patient was not on any other medication. 
He was fully aware of the dietary restrictions, to which he strictly adhered, and did not use alcohol or street drugs. However, after the tapering down of his daily dose of tranylcypromine was nearly complete, he decided - for the first time in years - to use khat again. The patient was familiar with functional headaches, but not with migraine. After chewing an initial portion of eight leaves, a sudden severe headache made him abort the session and report to the local emergency room. Upon arrival, he reported the headache to be occipitally located, radiating to the forehead, with nausea and hypersensitivity to the lights in the examination room. He stated that he did not have a "snapping" sensation prior to the onset of the headache. His speech was slow and he had difficulty finding words. He was sweating profusely; his blood pressure was 150/90 $\mathrm{mmHg}$ and his pulse $80 \mathrm{bpm}$. On further examination, no other abnormalities were found; no fever, and no neck stiffness or other neurological signs. The laboratory results were initially negative, but after a few days, traces of a recent subarachnoid hemorrhage were observed in the spinal fluid. This confirmed the results of an angiogram and a CT scan showing subarachnoid hemorrhage at the right frontal convexity in the absence of an intracerebral aneurysm. Upon injection of contrast fluid the headache recurred, but the patient eventually recovered completely without residual symptoms. His diagnosis at discharge was Reversible Cerebral Vasoconstriction Syndrome (RCVS). The etiology of this syndrome is unknown, although it is associated with sympathomimetics (Werring, 2010). As his daily dose of psychiatric medication was very low, the neurologist disregarded this as a possible cause.

\section{Discussion}

The active ingredient of khat is cathinone, a hydrophobic substance structurally similar to amphetamine and noradrenaline. It is easily absorbed through the mucosa of the mouth and the gastric wall and also passes the blood-brain barrier without difficulty. In the central nervous system, it enhances the release of dopamine and noradrenaline and possibly inhibits their subsequent re-uptake. This resembles the effects of amphetamine (Brenneisen et al., 1990; Al-Hebshi \& Skaug, 2005).

Pharmacological studies have advised against simultaneous use of khat and irreversible MAO inhibitors (Cox \& Rampers, 2003; Ricken et al., 2017). In addition, prolonged heavy use of cathinone has been related to cerebrovascular incidents (Mujlli et al., 2005; Al-Mottarrieb et al., 2010). MAO inhibition in the intestinal wall and the liver enhances the biological availability 
of cathinone by increasing the rate at which amphetamine-like substances are absorbed.

Upon their arrival in the synaptic cleft, amphetamine-like substances trigger the release of catecholamines from presynaptic vesicles. Due to the cumulative effect of irreversible MAO inhibition, these vesicles store considerably larger amounts of dopamine and noradrenaline than usual, even in patients who are on a low dose of tranylcypromine (Felner \& Waldmeier, 1979; Ulrich et al., 2017).

Release of such amounts of catecholamines into the synaptic cleft causes a hypertensive crisis, with tachycardia, mydriasis, and diaphoresis; intracerebral hemorrhage is a possible complication. Although the psychiatric medication of the patient was eliminated as a possible cause because of its low dose, the combined effects of irreversible MAO inhibition and an amphetamine-like substance may well be an explanation of the course of events in this case (Sjöqvist, 1965). However, as tranylcypromine interferes with the laboratory analysis of khat and amphetamines, the possibility that the patient used larger amounts of these substances than he reported cannot be ruled out (Marin et al., 2016).

\section{Tranylcypromine in patients with a background of migration}

This case illustrates an area of concern in the pharmacotherapeutic management of migrants. A prudent approach for psychiatrists would be to routinely inquire about the use of traditional herbs and substances and discuss their possible effects with patients. For patients with poor proficiency in Dutch, professional dialogue interpreters are essential for such discussions (KNMG, 2014). Unfortunately, the deployment of interpreters in mental health is no longer supported by the Dutch government (Triemstra et al., 2016). Persons seeking asylum are exempted from these recent regulations, but particularly in this target group, dialogue interpreting does not always result in a valid medical history. Mental health complaints are common in these patients, but in the context of an unfavorable asylum procedure, a person seeking asylum may be tempted to exaggerate the severity and persistence of psychiatric symptoms. In case of doubt, clinicians can use symptom validity tests to screen the validity of symptom reports (for examples, see Van der Heide \& Merckelbach, 2016, 2017). The uncertain validity of self-reported symptoms is an additional reason to be conservative in the prescription of medications like tranylcypromine. 


\section{Conclusion}

Simultaneous use of khat and tranylcypromine may result in a hypertensive crisis with possible severe consequences, such as cerebral hemorrhage. It is therefore prudent for psychiatrists to inquire about the use of traditional substances. If the medical history does not provide sufficient clarity, caution should be exercised in prescribing an irreversible MAO inhibitor. 



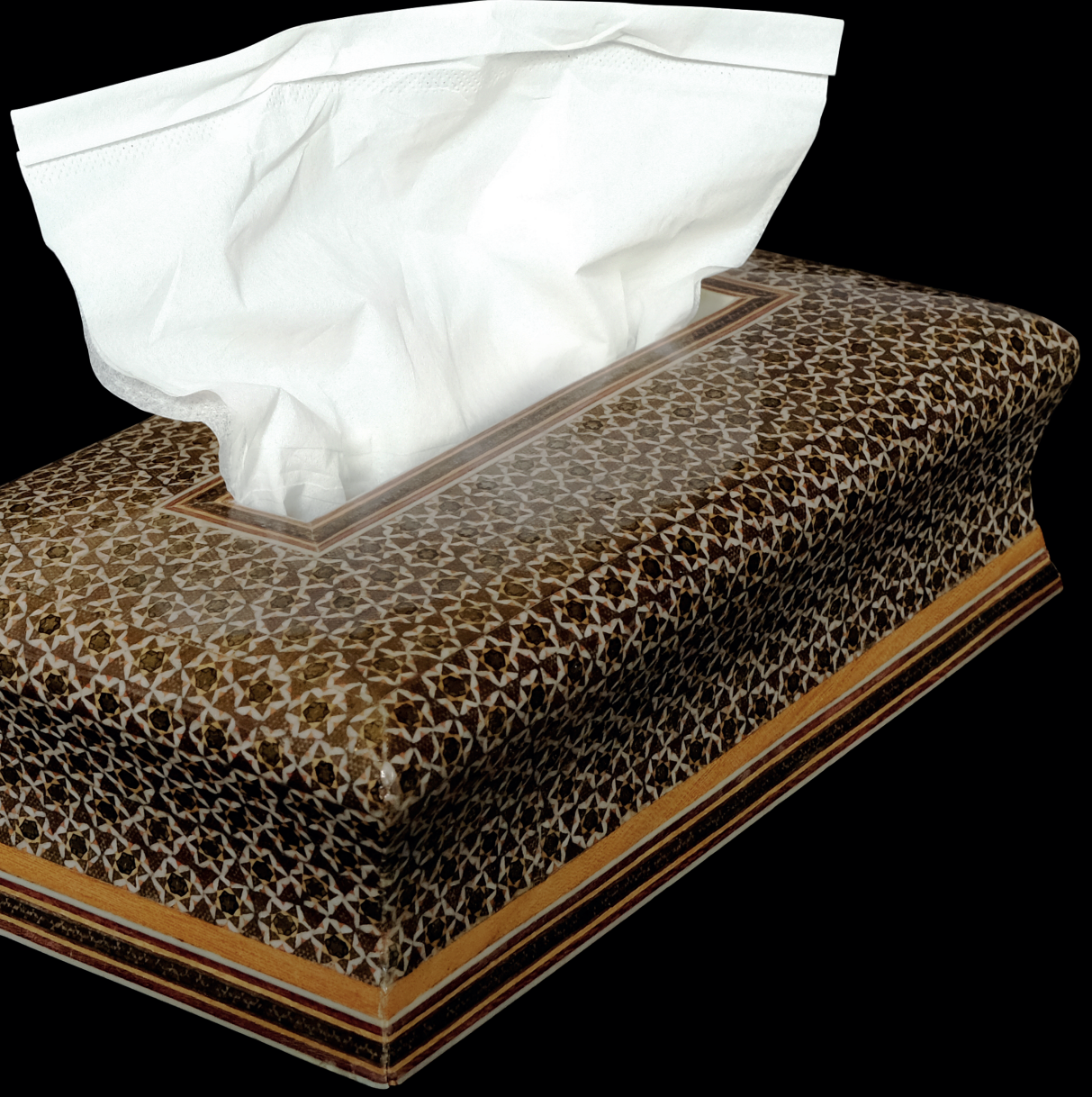




\section{4}

\section{VALIDITY OF SYMPTOM REPORTS OF ASYLUM SEEKERS IN A PSYCHIATRIC HOSPITAL: A DESCRIPTIVE STUDY}

Van der Heide, D., \& Merckelbach, H. (2016). Validity of symptom reports of asylum seekers in a psychiatric hospital: A descriptive study. International Journal of Law and Psychiatry, 49, 40-46. https://doi.org/10.1016/j.ijlp.2016.05.007 


\section{Abstract}

Our study involved three samples ( $n=85 ; n=38$, and $n=27$ ) of asylum seekers in a Dutch psychiatric hospital. We looked at how often they reported severe dissociative episodes (i.e., not recognizing oneself in a mirror; seeing traumatic images in a mirror) and whether these symptoms were related to deviant performance on Symptom Validity Tests (SVTs), notably items from the Structured Inventory of Malingered Symptomatology (SIMS; Widows \& Smith, 2005) and a forced-choice task modeled after the Morel Emotional Numbing Test (MENT; Morel, 1998). We also examined whether poor language proficiency and the presence of incentives to exaggerate symptoms might affect scores on SVTs. Dissociative target symptoms were reported by considerable percentages of patients (27-63\%). Patients who reported these symptoms had significantly more often deviant scores on SVT items compared with those who did not report such symptoms. With a few exceptions, deviant scores on SVT items were associated with incentives rather than poor language skills. We conclude that the validity of self-reported symptoms in this target group should not be taken for granted and that SVTs may yield important information. 


\section{Introduction}

Differences in culture and language complicate diagnostic decision making in psychiatry and may therefore affect its outcome negatively (Gara et al., 2012). Some authors have argued that it is below professional standards when clinicians evaluate patients whose language they do not speak (Artiola i Fortuni \& Mullaney, 1998). While this may be true in a general sense, clinicians confronted with asylum applicants who present with urgent mental problems often have to rely on on-the-spot translations by professional interpreters (Lustig, 2008; Reko et al., 2015).

Apart from cultural and language factors, intentional over-reporting may distort symptom reports and ultimately diagnostic judgment (Meffert et al., 2010). That is, asylum applicants in countries such as the Netherlands may have positive incentives to exaggerate their mental health problems; if their case for political asylum has been rejected, a refugee status may still be granted for medical reasons. Even after a refugee status has been granted, the ex-asylum applicants may claim special benefits (e.g., housing and family reunion) for people with mental vulnerabilities. Thus, some asylum applicants may have a motive to overreport symptoms. The other side of the coin is that reporting psychiatric symptoms during asylum-seeking procedures might be associated with negative incentives (e.g., compulsory hospital admission and accordingly limited freedom of movement). Meanwhile, little is known about the prevalence of symptom over-reporting among asylum applicants who present with psychiatric problems. Some authors have argued that malingering among asylum applicants is rare (Lustig, 2008), whereas others opine that malingering is a distinct possibility that should be taken into account (Morgan, 2007). Curiously enough, in the extant literature on, for example, post-traumatic stress symptoms in asylum seekers there is no example of a study in which researchers tried to differentiate between genuine symptom reports and symptom over-reporting. A case in point is the study of Kissane et al. (2014) who conducted interviews to assess post-traumatic stress symptoms in asylum seekers who reported psychological problems. More than half of the patients apparently suffered from severe post-traumatic symptomatology, and yet this statistic is difficult to interpret because the authors did not rule out symptom exaggeration. While Kissane et al. (2014) do acknowledge the cross-cultural validity problems that may arise when administering an interview to patients originating from Africa and Asia, they do not mention symptom exaggeration as a source of potential bias.

One way to assess the validity of symptom reports is by administering items that 
check for a tendency to claim implausible symptoms and deficits. There is now an extensive literature on so-called Symptom Validity Tests (SVTs) in the forensic context (see for a review e.g., Sollman \& Berry, 2011), but little research has been done on their utility in a cross-cultural context such as evaluating the symptoms of asylum applicants. However, the few cross-cultural studies that have been conducted suggest that SVTs may contribute to diagnostic accuracy in this domain (Salazar et al., 2007; Montes \& Guyton, 2014; Cirlugea, 2014). Still, so far, no study has made an attempt to differentiate between language difficulties and intentional overreporting as contributory factors to poor validity of symptoms reported by asylum applicants (see also Drob et al., 2009).

The current study explored the utility of typical SVT items in the diagnostic evaluation of asylum applicants who had been admitted to a psychiatric hospital. In this paper, asylum applicants may refer to refugees as well. In the Dutch system, refugees are asylum applicants who are granted a refugee status. In the first sample, we conducted with the help of professional interpreters an open interview, to examine how often asylum applicants admitted to a psychiatric hospital endorse specific depersonalization symptoms. We focused on symptoms that many clinicians assume to reflect a history of trauma (Friedman et al., 2011). In samples 2 and 3, we focused on similar symptoms and explored whether they are related to over-endorsement of implausible symptoms. We also tested whether limited proficiency in the language of the host country (Dutch) may be responsible for such over-endorsement.

Our study was exploratory in nature and the two main questions that we wanted to address were as follows: are severe dissociative symptoms reported by asylum seekers in a psychiatric hospital associated with deviant scores on SVT items? And to what extent does deviant SVT performance reflect poor language skills or incentives to exaggerate?

\section{Method}

\section{Patients}

Sample 1. Sample 1 consisted of a consecutive case series of 85 asylum seekers (60 men), who had been admitted to a clinical facility specialized in the treatment of asylum applicants with severe mental disorders in the Netherlands. This facility offers non-forensic, inpatient treatment only (32 beds) and is part of a larger general psychiatric hospital. Mean age was 37.3 years ( $S D=10.9$; range $18-61$ years). A total of 28 patients $(33 \%)$ originated 
from the Middle East, 23 (27\%) from the former USSR, 23 (27\%) from Africa, seven (8\%) from former Yugoslavia, and four (5\%) from East Asia. A majority (67\%) was reported to have a history of past psychotic episodes, and 27 patients (32\%) were still considered psychotic upon referral to the facility.

Sample 2. Sample 2 involved a consecutive series of 38 asylum seekers (30 men), who were treated in the same facility, after excluding three patients who were too disorganized to undergo testing and another two who refused informed consent. Mean age in this sample was 35.2 years ( $S D=11.7$; range $18-61$ years). In this sample, six patients (16\%) came from the Middle East, 12 (32\%) from the former USSR, 16 (42\%) from Africa, three (8\%) from former Yugoslavia, and one (3\%) from East Asia. Eight patients (21\%) had a poor proficiency in Dutch, 14 (37\%) had obtained an intermediate level of proficiency in that language, and 16 (42\%) were advanced students, as determined by a set of widely used criteria (see below). Also, 20 patients (53\%) had a positive incentive to over-report symptoms, 13 (34.2\%) had no incentive, and five (13.2\%) had a negative incentive as judged by an independent review of their case files (see below).

Sample 3. Sample 3 consisted of a consecutive series of 27 asylum seekers ( 19 men) from the same facility, after three patients were excluded because of severe mental disorganization and another two because they refused informed consent. Their mean age was 34.5 years (SD = 9.7; range $20-51$ years). In this sample, eight patients (30\%) originated from the Middle East, three (11\%) from the former USSR, 11 (41\%) originated from Africa, one (4\%) from former Yugoslavia, and four (15\%) from East Asia. Ten patients (37\%) had a poor proficiency in Dutch, nine (33\%) an intermediate proficiency, and eight (30\%) a good proficiency. Furthermore, 12 patients (44\%) had a positive incentive to over-report symptoms, nine (33\%) no incentive, and six (22\%) a negative incentive.

The study was conducted between 2005 and 2009. All patients admitted to the facility were included, except for those who refused informed consent or who were too disorganized to give such consent. The first sample was recruited two years before data in the second and the third sample were collected. Thus, there was no overlap in patients between sample 1 and samples 2 and 3 . The time interval between data collection in samples 2 and 3 was several weeks. Thus, when recruitment of sample 3 began, eight patients from sample 2 were still admitted in the clinic. Accordingly, they were included in sample 3 as well. The combined samples 2 and 3 consisted of 57 asylum seekers ( 45 men) with a mean age of 35.4 years (SD = 10.9; range $18-61$ years). Of these 13 patients (23\%) originated from the Middle East, 13 
(23\%) from the former USSR, 24 (42\%) from Africa, three (5\%) from former Yugoslavia, and four (7\%) from East Asia. Sixteen patients (28\%) had a poor proficiency in Dutch, 19 (33\%) an intermediate proficiency, and 22 (39\%) a good proficiency. Furthermore, 28 (49\%) had a positive incentive to over-report symptoms, 22 (39\%) no incentive, and seven (12\%) a negative incentive.

\section{Measures}

Open interview (sample 1). The purpose of the open interview was to explore on which scale patients reported the so-called signe du miroir (i.e., the inability to recognize oneself in a mirror) and related depersonalization symptoms. Ever since the French psychiatrist Paul Abély (1927) described the signe du miroir, it has been regarded as a severe form of depersonalization. Some authors concede that this symptom often precedes a psychotic breakdown (Abély, 1927; Goedhart \& Sno, 2014) and is related to a traumatic history (Friedman et al., 2011). Although the signe du miroir was first described by French psychiatrists, it has been reported for nonWestern psychiatric samples as well (Yu-Fen \& Neng, 1981). This symptom had the special attention of psychiatrists involved in the care for asylum applicants in the facility, because psychotic episodes require additional treatment effort. With these considerations in mind, the first author (DvdH) asked asylum applicants in the context of routine psychiatric evaluations about any particular experiences with mirrors during their admission. Questions were ad hoc translated by professional interpreters, who were either present in person or provided their services over the phone.

Implausible symptoms (samples 2 and 3). We used items derived from the Structured Inventory of Malingered Symptomatology (SIMS; Widows \& Smith, 2005) to assay the tendency to exaggerate symptoms. In its original form, the SIMS is a self-report instrument designed to screen for exaggeration of neurocognitive and psychiatric complaints. Basically, it consists of 75 true-false items that describe atypical and rare symptoms and experiences (e.g., "There is a constant ringing in my ear"; "The voices that I hear, have never stopped since they began"). A clear advantage of SIMS items is that they are easy to understand. There are five subscales, each containing 15 items, which address commonly feigned conditions: amnesia, neurologic impairment, psychosis, affective disorders, and low intelligence. After recoding some items, endorsed symptoms are summed to obtain a total SIMS score, with higher scores indicating more symptom over-endorsement. Previous studies recommended a cut-off of 16 for a comprehensive assessment of feigning (Merckelbach \& Smith, 2003). Van Impelen et al. (2014) summarize psychometric data indicating that the internal consistency of the SIMS is satisfactory (with 
Cronbach's alpha's ranging from 0.80-0.96), its test-retest stability sufficient ( $r$ 's $=0.72-0.97)$, and its ability to discriminate between symptom exaggeration and honest responding fairly effective (with sensitivities varying between $0.75 \%$ and $100 \%$ ).

For the purpose of the current study, some items of the Dutch research version of the SIMS (Merckelbach et al., 2001) were adapted. For example, after consultation with certified translators, the item pertaining to the queen of Holland was rephrased as follows: "The prophet of Allah is called Mohammed" (for patients with a Muslim background) and "The mother of Jesus is called Mary" (for patients with a Christian background).

For sample 2, two items about symptoms that had been found to be important during the open interview (sample 1; see above) were embedded as additional items in the list of SIMS items. These additional items were: "Some people have the experience of looking in a mirror and not recognizing themselves. Does this happen to you?" and "When I look at myself in the mirror, I think about terrible things that happened in my past". After instructions had been given and had been translated, the SIMS symptoms and the additional items were read out aloud. This was done by a fourth year psychology student for sample 2 and by the first author for sample 3. Items were presented at a comfortable pace, and each item was translated by professional interpreters, who also interpreted the answers of the patients (either a yes or a no).

Forced-choice task (samples $\mathbf{2}$ and 3). Patients were administered a forced-choice task closely modeled after the Morel Emotional Numbing Test (MENT; Morel, 1998). The task aims to detect response distortion in the assessment of trauma-related problems. We used a version that was developed by Geraerts et al. (2009), who observed in their sample of Croatian war veterans that high error levels were highly effective in differentiating between treatmentseeking and compensation seeking veterans (sensitivity: 92\%; specificity: 96\%). Briefly, the task comprised 20 colored slides of ten facial expressions posed by a man and a woman. Their expressions reflected happiness, frustration, sadness, anger, fear, calmness, surprise, shyness, confusion, and sleepiness. The slides were presented on a computer screen along with simple words that described emotional expressions (e.g., "happy"; "angry"). In a first series of 20 trials, patients saw one expression on the computer screen and had to indicate which of two words (e.g., "happy" versus "surprised") described the facial expression. In a second run of 20 trials, patients viewed two slides of different expressions, and they were given only one word; their task was to identify the expression that best matched the word. In a final run of 20 trials, patients were shown two slides and were given two words; slides and words had to be matched. The tests were conducted by the first author. During the test procedure, a 
professional interpreter was present and assisted with translating the instructions and the key verbal labels.

Before they underwent the task, patients were told that emotional numbness is a prominent symptom of trauma-related problems and that this may cause people to have difficulties with the recognition of facial expressions. The rationale behind this instruction is that individuals who want to overstate trauma-related symptoms may intentionally produce more errors during the forced-choice procedure. Errors were summed across the three runs. Morel (1998) recommended a cutting score of nine errors, with scores above this level raising the suspicion of symptom over-reporting. In the current study, we adopted the cutting score of nine errors (see also Geraerts et al., 2009). Compared with the open interview or the symptoms of the SIMS, forced-choice tasks such as the MENT require only minimal effort to translate test items, which makes them interesting in cross-cultural contexts. Another reason to employ this type of forced choice task is that it relies on the ability of respondents to identify facial expressions of basic emotions. Ever since Darwin (1872) and the pioneering work of Ekman and Friesen (1971) we know that expressions of basic emotions have their roots in biology rather than in culture.

Dissociative experiences (sample 3). Patients were given the items of the Dissociative Experiences Scale (DES-II; Bernstein \& Putnam, 1986). The DES is a self-report scale that requires participants to indicate on $100 \mathrm{~mm}$ visual analogue scales (VAS; anchors: 0 = never; 100 = always) to what extent they experience 28 dissociative experiences in daily life (amnesia, depersonalization, absorption). In a meta-analysis, van IJzendoorn and Schuengel (1996) provide evidence for the sound psychometric properties of the DES. Summarizing the findings of a large pool of studies, among which studies that administered the DES to African American, Caucasian, and Hispanic war veterans, these authors conclude that the overall internal consistency of the DES is good (mean Cronbach's alpha $=0.93$ ), while the test-retest stability is satisfactory (r's = 079-0.90). The DES items were read aloud by a sixth year medical resident, after which each item was translated by a professional translator, who also interpreted the answers of the patients. One of the two target symptoms that had been explored during the open interview in sample 1 - not recognizing yourself in a mirror - is listed as a separate item in the DES (i.e., item 11). Therefore, there was no need to add this item to the list of SIMS symptoms, as we did in sample 2. The second target symptom was added as an extra item to the DES and rephrased to fit the original description better, which specifically included seeing images ("Some people have the experience of looking in a mirror and seeing images of people or events from the 
past"). DES items as well as the additional item were rated by patients on $0-100 \%$ VAS ( $0=$ zero percent of the time, $100=100 \%$ of the time). The target symptoms - not recognizing yourself in a mirror and seeing images from the past in mirrors- were considered to be present when they were endorsed at a minimum of $10 \%$.

Because the pertinent depersonalization symptoms were gauged in a different way in sample 2 and 3 - within the context of the SIMS items and within the context of the DES items, respectively - we decided to present the data collected in both samples separately.

\section{Procedure}

The SIMS items, the forced-choice task, and DES items were administered to patients after the hospital decided to introduce Routine Outcome Monitoring (ROM). SIMS items and forced-choice task were included to serve as an additional quality check on self-reported symptoms. Before SIMS items and forced-choice task were administered, patients were informed that the tests were employed to assess the validity of symptom reporting in their target group. They were told that if the test results indicated a poor validity in their case, the conclusion would be that Western-style psychological tests would not provide useful information and that their diagnosis was to be based on additional interviews and observations. Only patients who gave informed consent for anonymous use of their data for scientific purposes were included. The study was approved by the Central Committee on Research Involving Human Subjects (CCMO). SIMS items, forced-choice task, and dissociative experiences items were presented in a counterbalanced fashion as much as possible.

Patients in samples 2 and 3 were independently assessed by Dutch language teachers of the hospital. On the basis of fixed criteria (Meijer \& Noijons, 2008), these experts categorized patients into speakers with a poor proficiency in Dutch, an intermediate proficiency, and an advanced proficiency. These proficiency levels were taken to be a reasonable proxy for the degree to which patients would need the help of professional interpreters during diagnostic procedures.

For samples 2 and 3, social workers of the hospital independently evaluated asylum applicants' files for the presence of incentives. The social workers were blind as to patients' performance on the SIMS items and the forced-choice task. Three groups were formed: patients with a positive incentive to over-report symptoms, patients who had as many positive as negative incentives (referred to as "no" incentive group), and patients with a negative incentive. Patients were assigned one point for each circumstance promoting over-reporting, 
notably: 1. involvement in an ongoing asylum procedure; 2 . seeking a temporary refugee status issued for medical reasons; and/or 3. involvement in any other current procedure requiring a medical report indicating medical necessity, urgency or exemption (e.g., request for family reunion although the patient is not able to generate the necessary income demanded by Dutch law; a request for urgent change of housing or special housing arrangements; a request to be exempted from the demand to pass the language test in the naturalization procedure). For each circumstance that would make intentional over-reporting less likely, the raters subtracted a point. Such circumstances would be: 1. a compulsory nature of the present admission; 2 . involvement in any current procedure requiring a medical report indicating improved functioning or decreased need for medical treatment or scrutiny (e.g., a child custody procedure, a request for voluntary repatriation). Patients with one point or more were considered to have a positive incentive, and patients with minus one point or less to have a negative incentive.

Importantly, neither the psychiatrist who conducted the open interviews, nor the psychiatrists or students who presented the SIMS items, forced-choice task, and DES items were aware of the language proficiency level of applicants. Neither had they information regarding patients' incentive scores. On the other hand: both the psychiatrist and the students were involved in the treatment of the patients. So they had some background information about patients and blinding was not complete.

\section{Data analysis}

We used descriptive statistics to study the prevalence of severe dissociative target symptoms, SIMS symptoms, and errors on the forced-choice task in our samples. Depending on whether data were skewed or evenly distributed, we employed one-way Analyses of Variance (ANOVA's) or Kruskal-Wallis and Mann-Whitney $U$ tests to compare language proficiency and incentive groups with regard to their endorsement of SIMS symptoms and their errors on our forced choice task. 


\section{Results}

\section{Prevalence of target symptoms in sample 1}

Thirty patients (35\%) reported during the open interview the experience of not recognizing themselves in mirrors. That is, they regularly had at least doubts as to whether the reflection in the mirror was their own. Twenty three patients $(27 \%)$ said that they had experienced seeing images in the mirror with a traumatic content, usually pertaining to aversive events from their personal past or related to loved ones who passed away during traumatic circumstances. Both symptoms had in common that they appeared to be induced by stress and that they had a tendency to occur during the night. Patients reporting these symptoms also said that they tended to avoid mirrors, except for five patients who said that they obsessively scrutinized their appearance in mirrors. The capacity to test the reality of these experiences remained intact in all patients, except for a minority who met criteria for a self-misidentification syndrome (four patients), or for a paranoid delusion towards mirrors (five patients). Thus, the results of the open interview showed that about one third of the patients reported the signe du miroir and related dissociative experiences.

\section{Endorsed SIMS symptoms and forced-choice performance in sample 2}

Eleven respondents said they were not able to choose between "true" or "false" for a total of 71 SIMS symptoms. These missing values were treated in a conservative way, i.e., as indicating non-endorsement. The internal consistency of the SIMS symptoms was satisfactory. Thus, Cronbach's alpha was 0.95 for the total set of items and ranged from 0.56 (affective disorders subscale) to 0.86 (psychotic disorders subscale) for separate subscales. As a check on inter-rater reliability of the SIMS items, eight patients were tested twice (with time intervals in between of several weeks): by the first author ( $\mathrm{DvdH}$ ) and by a test assistant. In this subgroup, test-retest scores correlated significantly: $r=0.91, p<0.05$.

The mean endorsement rate of SIMS symptoms was 35.1 (SD = 15.7), 95\% Cl [29.7, 40.5]. A majority of patients (87\%) had scores that exceeded the original cut-off of 16 . Patients exhibiting poor Dutch proficiency $(n=8)$ endorsed on average 44.1 SIMS symptoms (SD $=8.8$ ); those with an intermediate proficiency $(n=14)$ obtained a mean score of $34.1(S D=18.3)$, and those with a good proficiency $(n=16)$ had a mean endorsement rate of $31.5(S D=15.0)$. The SIMS data in this sample were normally distributed. A one-way ANOVA that compared the mean SIMS scores of the three groups failed to attain significance: $F(2,35)=1.84, p=0.17$. Patients with 
positive incentives $(n=20)$ had a mean SIMS score of $44.2(S D=11.3)$, those with a negative incentive $(n=5)$ had a mean score of $13.8(S D=6.2)$, while those with "no" incentives $(n=13)$ had an intermediate score of $29.3(S D=13.6)$; a one-way ANOVA yielded a significant effect, $F(2,35)=15.9, p<0.001, \eta^{2}=0.48$. Follow-up t-tests made it clear that the positive incentive group had a higher SIMS endorsement rate than the negative incentive group ( $t(23)=5.7, p$ $<0.05)$ and the group with no incentives $(t(31)=3.4, p<0.05)$; the negative incentive group had lower endorsement levels than the group without incentives $(t(16)=2.4, p=0.05)$.

There were no missing data for the forced-choice trials in this sample. Cronbach's alpha was 0.96. The mean error score on our forced-choice task was 17.7, 95\% Cl [12.6, 22.8]. In total, $58 \%$ of the patients scored above the cut-point of nine errors. All patients who scored above the cut-off for the SIMS, also did so for the forced-choice task. The correlation between the number of forced-choice errors and endorsement rate of SIMS symptoms was $r=0.65, p=0.01$.

Patients with a poor proficiency had on average 32.3 errors $(S D=13.1)$, those with an intermediate proficiency had 17.0 errors $(S D=11.7)$, and patients with a good proficiency made 11.1 errors $(S D=13.7)$. The forced-choice data in this sample were not normally distributed (Shapiro-Wilk $p$ 0.001). A Kruskal-Wallis test showed that group differences were significant: $p<0.01$. Patients with a positive incentive had 27.0 errors $(S D=14.8)$, those with a negative incentive had on average 5.6 errors $(S D=3.5)$, while patients with "no" incentive had 8.2 errors $(S D=5.5)$. A Kruskal-Wallis test indicated that these group differences were significant: $p<0.001$.

The additional item on having difficulties recognizing oneself in a mirror was endorsed by 14 patients (37\%). The item on thinking of terrible past events when looking into a mirror was endorsed by 24 patients (63\%). Table 1 shows how these symptom reports relate to SIMS and forced-choice scores. As can be seen, patients who said that they had difficulties recognizing themselves in mirrors endorsed more SIMS symptoms, $t(38)=4.17, p<0.01$, and had more forced-choice errors, Mann-Whitney $U: p<0.01$, compared with patients who did not report this symptom. A similar pattern emerged for reporting thoughts about terrible past events when looking into mirrors: $t(38)=7.43, p<0.01$ and Mann-Whitney $\mathrm{U}: p<0.01$, respectively.

We also looked at percentages of patients who failed the SIMS and forced-choice cut-points and basically replicated the pattern described above, but for one exception. Those who reported not being able to recognize themselves in mirrors more often failed on the forced-choice task, but not on the SIMS items: Fisher exact $p$ 's $=0.02$ and 0.14 , respectively. For thinking about terrible past events when looking into mirrors, both Fisher exact $p$ 's were $<0.01$. 
Table 1. Number of endorsed SIMS items and forced-choice errors of patients in Sample $2(n=38)$ who did (yes) or did not (no) report the target symptoms of depersonalization.

\begin{tabular}{lcccc}
\hline & $\begin{array}{l}\text { Not recognizing } \\
\text { oneself in mirror }\end{array}$ & \multicolumn{3}{c}{$\begin{array}{c}\text { Thinking about terrible } \\
\text { past events when } \\
\text { looking into mirror }\end{array}$} \\
\cline { 2 - 5 } & Yes $(n=14)$ & No $(n=24)$ & Yes $(n=24)$ & No $(n=14)$ \\
\hline Mean (SD) SIMS & $46.7(11.1)$ & $28.3(14.1)$ & $44.3(10.7)$ & $19.3(8.8)$ \\
$n(\%)>16$ SIMS & $14(100 \%)$ & $19(79 \%)$ & $24(100 \%)$ & $9(64 \%)$ \\
Mean (SD) Forced- & $29.1(15.1)$ & $11.1(10.1)$ & $24.0(15.5)$ & $7.1(3.8)$ \\
choice errors & $12(86 \%)$ & $10(42 \%)$ & $18(75 \%)$ & $4(28 \%)$ \\
$n(\%)>9$ errors & & & & \\
Forced-choice & & & & \\
\hline
\end{tabular}

\section{Endorsed SIMS symptoms and forced-choice performance in sample 3}

In this sample, the SIMS items were presented to patients by the first author; there were no missing data. Cronbach's alpha of the total set of SIMS symptoms was 0.96 and alpha's for subscales varied between 0.74 (affective disorders) and 0.91 (amnesia). The mean SIMS symptom endorsement rate was $27.0(S D=17.3), 95 \% \mathrm{Cl}[20.2,33.7]$. In total, 17 patients (63\%) scored above the cut-off of 16 symptoms. Endorsement rates were not normally distributed (Shapiro-Wilk $p<0.05)$. Mean score in patients with a poor proficiency was $33.4(S D=19.4)$. In patients with an intermediate proficiency this was $20.4(S D=14.0)$, while those with a good proficiency endorsed on average 26.5 symptoms $(S D=17.6)$. As indicated by a Kruskal-Wallis test, these group differences did not attain significance $(p=0.24)$. Mean symptom endorsement in patients with a positive incentive was $43.4(S D=9.2)$. Mean symptom endorsement in patients with a negative incentive was $11.7(S D=4.4)$, while those with "no" incentive attained a score of 15.2 (SD =10.2). A Kruskal-Wallis test showed that this difference was significant, $p<0.001$.

There were no missing forced-choice data and Cronbach's alpha for this test was 0.95. The mean error score was 12.4 (SD = 11.8), 95\% Cl [7.8,17.0]. In total, 11 patients (41\%) scored above the cut-off point of 9. The forced-choice data were not normally distributed (ShapiroWilk $p<0.01)$. The mean error score of patients with a poor proficiency was $17.7(S D=14.7)$. Those with an intermediate proficiency had on average $10.7(S D=10.0)$ errors and the error rate of those with a good proficiency was $7.8(S D=7.5)$. A Kruskal-Wallis test indicated that group differences in forced-choice errors were not significant: $p=0.19$. Mean error score of patients with a positive incentive was $21.2(S D=12.7)$. Patients with a negative incentive to over-report symptoms had on average 6.8 errors $(S D=3.3)$. For patients with "no" incentive, 
the mean number of errors was $4.8(S D=4.3)$. A Kruskal-Wallis test indicated that these group differences were significant, $p<0.01$.

Forced-choice errors and number of endorsed SIMS symptoms correlated at $r=$ $0.74, p<0.01$. All patients who scored above the cut-off point on the SIMS, also did so for the forced-choice test.

The mean score on the dissociative items of the DES-II was $22.6(S D=21.5)$. Cronbach's alpha was 0.97; there were no missing data. DES-II scores correlated both with endorsed SIMS symptoms and forced-choice errors: $r=0.79, p<0.001$ and $r=0.41, p<0.05$, respectively. The DES-II item on difficulties to recognize oneself in a mirror was endorsed by nine patients (33\%) for at least ten percent of the time (range: 20\%-100\%). The reported mean frequency of this experience in all patients was $19.6 \%$ of the time (SD $=32.6)$. Eight patients $(30 \%)$ endorsed the additional item on seeing images of past traumatic experiences in the mirror. The reported mean frequency of this experience in all respondents was $16.9 \%$ of the time (SD $=30.5$ ).

Table 2 gives rates of endorsed SIMS symptoms and forced-choice errors in the two symptom groups. As can be seen, the results replicate the pattern that is evident in Table 1. Thus, patients who had difficulties recognizing themselves in mirrors had higher SIMS scores and made more forced-choice errors than those who did not report this symptom (Mann-Whitney $\mathrm{U}: p<0.01$ and $p<0.01$, respectively). Likewise, those who reported this symptom tended to fail more often on the SIMS (Fisher exact $p=0.09$ ) and more often failed on the forced-choice task (Fisher exact $p=0.01$ ). Patients who reported seeing aversive images in mirrors attained higher error scores on both the SIMS and the force-choice procedure than those who did not report this symptom (Mann-Whitney $\mathrm{U}: p=0.01$ and $p=0.01$, respectively). Similarly, those who reported this symptom more often failed on the SIMS and the forced-choice task (Fisher exact $p^{\prime} s=0.01$ and 0.01 , respectively). 
Table 2. Number of endorsed SIMS items and forced-choice errors of patients in Sample $3(n=27)$ who did (yes) or did not (no) report the target symptoms of depersonalization.

\begin{tabular}{lcccc}
\hline & $\begin{array}{c}\text { Not recognizing } \\
\text { oneself in mirror }\end{array}$ & & \multicolumn{2}{c}{$\begin{array}{c}\text { Seeing images } \\
\text { from past in } \\
\text { mirror }\end{array}$} \\
\cline { 2 - 5 } & Yes $(n=9)$ & No $(n=18)$ & Yes $(n=8)$ & No $(n=19)$ \\
\hline Mean (SD) SIMS & $40.2(15.2)$ & $20.3(14.6)$ & $43.0(12.5)$ & $20.2(14.5)$ \\
$n(\%)>16$ SIMS & $8(89 \%)$ & $9(50 \%)$ & $8(100 \%)$ & $9(47 \%)$ \\
$\begin{array}{l}\text { Mean (SD) Forced choice } \\
\text { errors }\end{array}$ & $21.1(14.3)$ & $8.1(7.5)$ & $24.1(13.6)$ & $7.5(6.6)$ \\
$n(\%)>9$ errors Forced- & $7(78 \%)$ & $4(22 \%)$ & $7(88 \%)$ & $4(21 \%)$ \\
choice & & & & \\
\hline
\end{tabular}

\section{Discussion}

The results of the current study can be summarized as follows. Firstly, we found that considerable proportions (27-37\%) in samples of asylum applicants who were admitted to a psychiatric ward reported severe depersonalization symptoms. These are typically symptoms that would warrant asylum for medical reasons, but their prevalence is not out of line with rates found elsewhere in clinical settings (Foote et al., 2006; Friedl et al., 2000). Secondly, as a group, applicants had relatively high scores on our SVTs and many (41-87\%) failed on these instruments when the conventional cut-points were employed. Thirdly, SVT scores were more strongly associated with incentives to malinger than with poor proficiency in the host language. That is, positive incentives to malinger were associated with higher endorsement of SIMS symptoms and more errors on our forced-choice task modeled after the MENT (Morel,1998) ; poor proficiency in Dutch was only related to more errors on this forced-choice task- the SVT that was least dependent on interpretation.

There can be little doubt that asylum seekers form a highly vulnerable group, with high rates of psychopathology, notably trauma-related psychopathology (Reko et al., 2015). It is also clear that the psychopathology in this group is often evaluated in an unstructured way, i.e., without using standardized screening or diagnostic instruments, which raises diagnostic uncertainty (Maier et al., 2010). Our results should not be taken to imply that there are many malingerers among asylum seekers. Rather they suggest that a tendency to over-report symptoms is prevalent in this group, which speaks to the need to evaluate symptomatology in 
this group in a standardized and more controlled way. A tendency to over-report symptoms may reflect many underlying factors, one of which is illness behavior so as to access healthcare services (McColl et al., 2008). That is, symptom over-reporting does not exclude the presence of real pathology. We do know, however, that over-reporting may obscure diagnostic evaluations and result in greater health care utilization (e.g., Horner et al., 2014).

Several limitations of the current study should be noted. To begin with, the SVTS were introduced not for research purposes, but as an internal quality control during the implementation of ROM. Part of the staff who presented the tests were also involved in the treatment process, which means that blinding was not complete. However, data about the incentives and proficiency levels of the patients were gathered independently by other staff after the tests were completed.

Another limitation is that our samples were relatively small and therefore our results need replication, preferably with different SVTs. The SVTs that we used have been translated in other languages, but their validity has not been established for target groups with the highly diverse cultural backgrounds that are typical for asylum seekers in Western European countries. Because of this cultural diversity, we had to rely on an oral version of the SIMS that was translated in an ad hoc fashion by professional interpreters. Likewise, for giving the instructions of the forced-choice task to the patients, we relied on interpretation by professional interpreters. Thus, our SVTs were administered in a suboptimal way, and therefore mean scores and percentages of patients who failed these tests should be interpreted with caution. Relatedly, although translation of the items was done by professional interpreters, previous research has shown that interpreters might produce translation errors (Bot, 2005) and these may affect results.

The other side of the coin is that administering diagnostic tests with help of professional translators is routine practice in this clinic and in similar facilities for asylum seekers; our results are therefore a realistic reflection of diagnostic validity in clinical practice. Still, it would be a step forward if SVTs would be available that have pre-translated templates for various language groups, such that clinicians and researchers are less dependent on translators.

Although an ad hoc translation procedure was also used for the DES, this test- in contrast to the SVTs- has been tested in different cultural communities across several continents; its cross-cultural validity is therefore considered to be good (Fang et al., 2011; Zoroglu et al., 2002). The fact that prevalence of the "mirror sign" in the third sample of our study- where the DES was used- is comparable to its prevalence in the other samples does not support 
the notion that the cultural validity of the methods employed in these samples was inferior.

Unfortunately, in our samples, the various cultural and linguistic groups were represented by too few patients to run separate analyses for cultural subgroups. This is an important shortcoming of our study if only because traumatic experiences of Yugoslavian refugees, for example, may be expected to be tremendously different from those from East Asia or Africa.

A final limitation concerns the way in which the incentive potential was evaluated by social workers. The social workers inspected the files, thereby checking a restricted number of possible (positive and negative) incentives. Obviously, this is a crude approach, because incentives might be subtle (e.g., a desire to please the therapist) and need not to be documented in patient files. However, even with this crude approach we found deviant SVT performance to be related to the presence of positive incentives.

Our results, then, cast doubts on the validity of symptom reports about not being able to recognize oneself in mirrors and seeing aversive images in mirrors. Although the present analysis fails to confirm a large effect of language proficiency on SVT performance, we would overstate our case if we would argue that applicants who failed our SVTs are malingerers. For one thing, cultural issues may affect both symptom reports and performance on SVTs. What can be said with some confidence, though, is that - for whatever reason- the validity of these depersonalization symptoms cannot be taken at face value and that when asylum patients report them a thorough follow-up examination is in order. This is important because when therapists are under the impression that their asylum patients do have these symptoms, when in fact they do not, this may result in wrong treatment decisions (e.g., prescribing medication with potentially harmful side-effects).

\section{Conclusions}

Our study was exploratory in nature. Our attempt to differentiate between language proficiency levels and incentives to over-report might have been problematic because, for example, a tendency among the respondents to exaggerate cognitive impairment might have affected the evaluation of their proficiency in the host language. Still, our results suggest that when asylum applicants report during a free, unstructured psychiatric interview severe depersonalization symptoms, the validity of these symptoms cannot be taken at face value. Our study illustrates that this domain may benefit from the systematic administration of 
SVTs, although such instruments can by themselves never reveal whether or not a patient is intentionally over-reporting (Merten \& Merckelbach, 2013). Several position papers have recommended the use of SVTs in neuropsychological assessment, emphasizing that validity testing is crucial for an accurate interpretation of clinical data (Heilbronner et al., 2009). We would argue that much the same is true for psychiatric evaluations in asylum seekers.

For future research, relating SVTs to culturally validated self-report instruments instead of open interviews may further clarify the relative impact of differences in culture, language, and secondary gain expectations in this target group. 



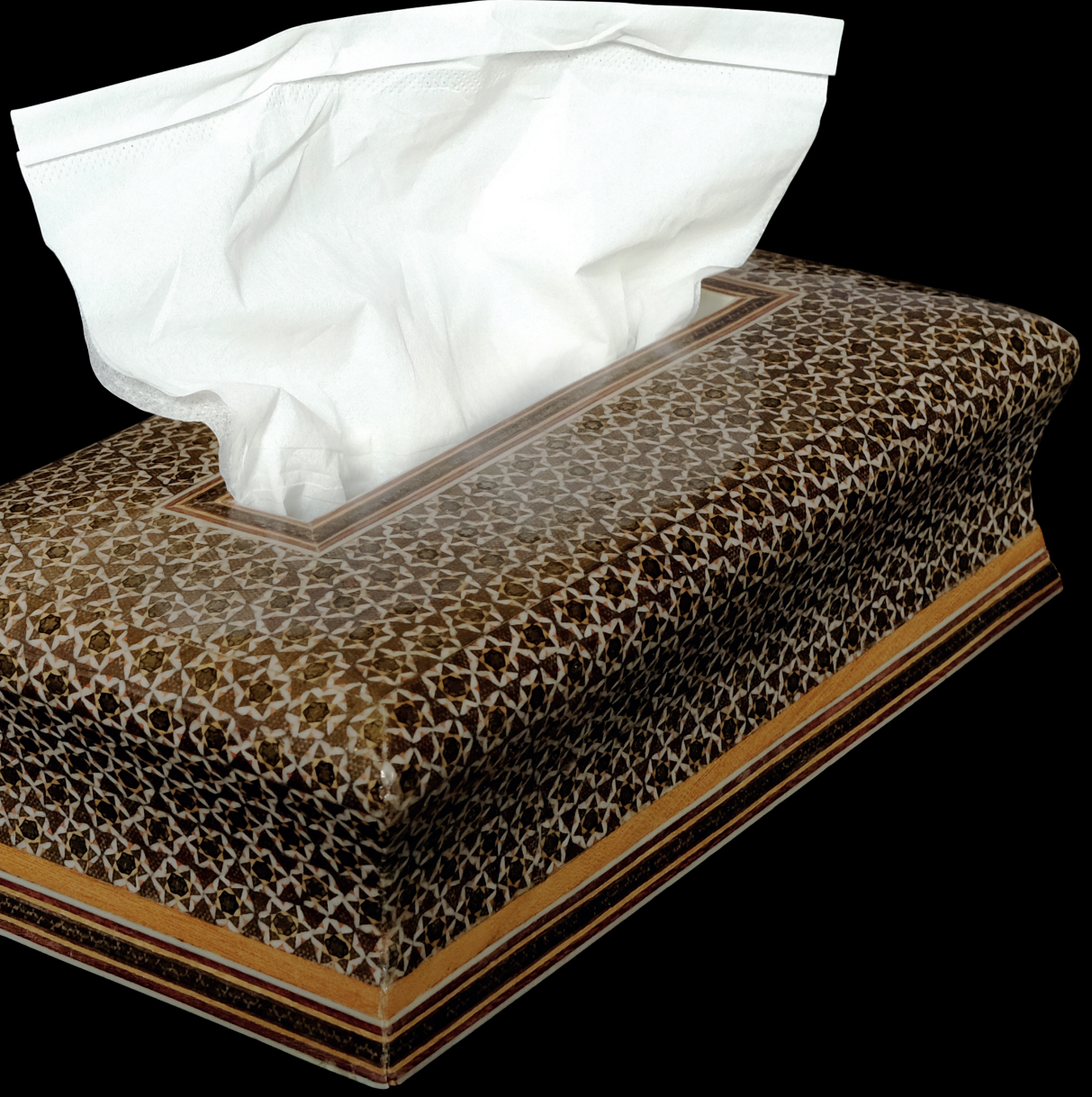




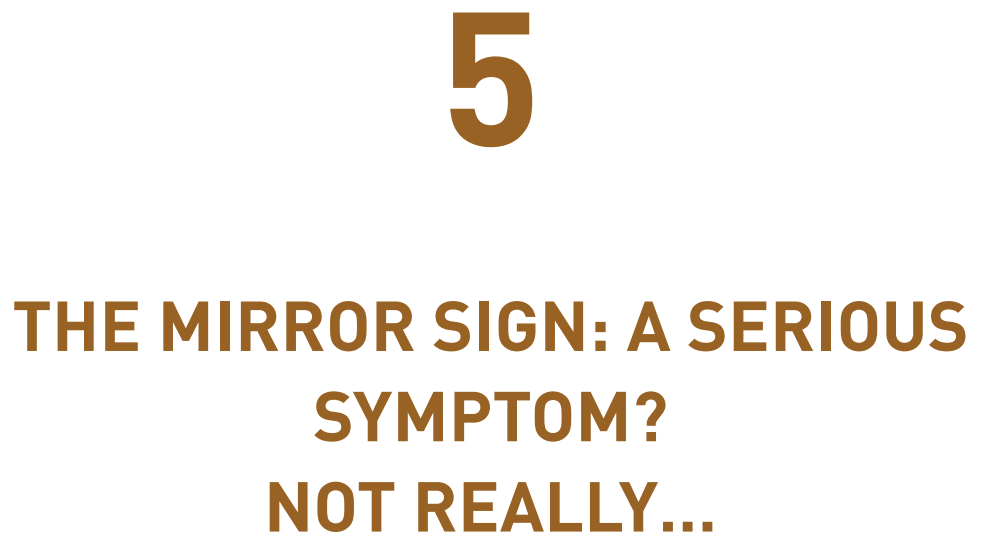

Published as:

Van der Heide, D., \& Merckelbach, H. (2016). Het spiegelteken: een ernstig symptoom? Nou, nee... Tijdschrift voor Klinische Psychologie, 2, 121-130.

https://vvkp.be/het-spiegelteken-een-ernstig-symptoom-nou-nee-douwe-van-der-heideharald-merckelbach 


\section{Abstract}

The loss of the capacity to recognize oneself in a mirror-the mirror sign - is considered to be a precursor to psychotic dysregulation. In any case, that is what the psychiatrist Lacan and his followers have argued. However, many Anglo-Saxon authors regard the mirror sign as a core symptom of dissociation. Based in part on three empirical studies, we took a closer look at both interpretations. In these studies, we found that too many respondents (university students) reported the mirror sign to regard it as an indication of an impending psychotic episode. Interestingly, a substantial number of students also reported seeing their own reflection constantly, an experience that appears to be the opposite of the mirror sign. Does this indicate careless responding on the part of the students? The importance of that question is underlined by our finding that self-report of the mirror sign was associated with a tendency to exaggerate symptoms. Outside the population of psychotic patients, this over-reporting of symptoms may well be the true significance of the mirror sign. 


\section{Introduction}

The most beautiful description of the mirror sign is the one given by Pirandello, laureate of the Nobel Prize for literature in 1927. In his novel Uno, nessuno e centomila (Pirandello, 1926), he describes a young man who becomes obsessed by his image in the mirror after his wife has made an innocent remark about his nose. He ultimately becomes psychotic after the face in the mirror loses its identity: according to the protagonist the reflection might as well be one face, no face, and hundred thousand faces (uno, nessuno e centomila).

When his novel was published, Pirandello was an internationally renowned author. A year after the appearance of the novel, a French psychiatrist, Abély, described a young man who became obsessed by his own image in mirrors just before the onset of his first psychotic episode (Abély, 1927). Abély's report was followed by reports from several other French psychiatrists with similar cases, and the mirror sign (signe du miroir) became recognized as psychiatric symptom - at least in Francophone psychiatric literature (Abély. 1930; Delmas, 1929; Ostancow, 1934). Remarkably, patients in whom the mirror sign is observed often tend to deny it. The consensus is that the mirror sign should be regarded as an early sign of an impending psychosis.

The mirror sign was also described in other countries, for example after the introduction of one-way mirrors to observe psychotic patients (Rosenzweig \& Shakow, 1937). But theorizing about the mirror sign took place mainly in France. In 1931, Wallon wrote about the phase in which children start to recognize themselves in mirrors as a pivotal episode in the development of the mind (Wallon, 1931). The psychoanalyst Lacan took this idea one step further by stating that the mirror stage (stade du miroir) marks the transition into the oedipal phase. Children learn to define themselves as separate entity (me), which immediately turns it into an integral part of the world of language (discours) of adults. In adult psychotic patients, the mirror sign thus marks the reverse development: a regression into a childlike, pre-oedipal world of experience, in which the self is no longer separate from others (Lacan, 1966, pp. 93-100). This notion became the cornerstone of Lacan's theory. After the publication of Ecrits (Lacan, 1966), he became internationally renowned as a psychoanalytic theorist.

\section{Signe du miroir compared to the mirror sign}

Children start to recognize themselves in their second year of life. This event is part of a developmental cascade that includes - as Lacan theorized - the linguistic ability to refer 
to oneself (me), along with skills such as empathy (Anderson, 1984). With the exception of a few species - some primates (Gallup, Anderson, \& Shillito, 2002), dolphins and orcas (Delfour \& Marten, 2001) and elephants (Plotnik, De Waal, \& Reiss, 2006) - animals are unable to recognize themselves in mirrors. Chimpanzees tend to lose this ability in old age (Povinelli, Rulf, Landau, \& Bierschwale, 1993), similarly to humans in the course of Alzheimer's disease (Biringer, Anderson, \& Strubel, 1988). This development is easily mistaken for a psychotic sign; in case of a "phantom boarder", socially isolated elderly patients complain about a mysterious intruder in their homes until it turns out that the intruder is their own image in the mirror (Hwang, Yang, \& Tsai, 2003). This specific type of cognitive impairment is also referred to as mirror sign in Anglo-Saxon medical literature, but this is different from the mirror sign as used in this paper, in which the cognitive component of self-recognition is still intact.

\section{The mirror sign as depersonalization}

Patients afflicted by the mirror sign tend to gesticulate or talk to mirrors, although some tend to avoid mirrors, for example by covering them with a piece of cloth. The experience underlying this behavior is that the image in the mirror no longer feels like one's own. The observed reflection does have a striking resemblance to one's own face, and yet appears to be somehow different, creating a sensation of estrangement - as if a doppelgänger is staring back from the mirror. The pre-reflective, affective aspect of self-recognition that links cognitive self-recognition to a sense of self is no longer present.

Apart from Lacan, several other authors offered an explanation for the mirror sign (Goedhart \& Sno, 2014). Based on the lack of emotional self-recognition, most have interpreted the sign as a type of depersonalization (Delmas, 1929; Galant, 1935; Gheorghiev, 2011; Ishida, 1954; Ostancow, 1934; Parnas \& Handest 2003), and more in general terms, a symptom of dissociation. For example, in The Stranger in The Mirror, a popular science book by Steinberg and Schnall (2001) about dissociative disorders, the "inability to recognize yourself in a mirror" (p. 53) is described as a core symptom. Remarkably, the direct opposite of the mirror sign "seeing oneself from a distance as if looking in a mirror" - is also regarded to be a dissociative phenomenon, known as autoscopy.

The fact that both mirror sign and autoscopy are regarded as dissociative symptoms could be an indication that the category of dissociative experiences is poorly demarcated. Indeed, both symptoms could share a common experience of identity confusion, about how one's identity must be distinguished from the outside world. Which leads us back to Lacan and 
more recent authors, who regard this feeling of estrangement from the self as an experience that heralds a major psychological crisis that would presumably not be encountered outside the realm of psychiatry. Unfortunately, the current literature is mainly based on psychiatric case reports, and little is known about know how often the mirror sign is reported by healthy respondents.

\section{The first sample: Results from the Dissociative Experiences Scale}

The frequently used Dissociative Experiences Scale (DES) asks respondents or patients to indicate the frequency of occurrence of 27 items on a semi-continuous scale $(0 \%=$ never, $100 \%$ = always). Item 11 reads as follows: "Some people have the experience of looking in a mirror and not recognizing themselves." (Bernstein \& Putnam, 1986). That description is fairly close to our current understanding of the mirror sign. To determine its prevalence in a healthy population, we performed a study in which 231 students (average age 20 years; range: 18-27 years) completed the Dutch paper-and-pencil version of the DES, along with several other questionnaires that are not relevant to this discussion. Participants were recruited through advertisements to participate in a study about psychological problems. They were compensated with a gift voucher or a study point in case of psychology students. The test sessions were conducted in groups of 10-15 persons in the presence of a test supervisor who could answer questions. The study was approved by the standing ethical committee of the faculty of Psychology and Neuroscience of the University of Maastricht.

The questionnaires of 18 students were excluded because of missing data. Cronbach's alpha of the DES was 0.94; DES item 11 had a mean score van 8.7 (SD =16.6), which appears to be low at first sight. On the other hand: about $10 \%$ of the respondents reported experiencing the mirror sign at least 30\% of the time (see Table 1). Our sample does not represent the population at large (most participants were students who responded voluntarily to an advertisement), but similar proportions were reported in the Canadian population-based study by Ross et al. (1991). This prevalence in a healthy sample appears to contradict the notion that the mirror sign heralds a psychotic breakdown. The lifetime prevalence of psychotic disorders is considerably lower, about 3\% (Perala et al., 2007). This figure refers to psychotic episodes resulting in a psychiatric admission - the type Abély (1930) was referring to.

Item 11 of the DES is part of a subscale that probes for depersonalization. According to some authors, it is a prominent symptom of dissociation (Steinberg \& Schnall, 2001). Others disagree. For instance, Simeon et al. (1998) asked patients with depersonalization 
disorder whether they knew the item out of their own experience; these patients regarded the mirror sign as "rare or bizarre". Perhaps this is the reason that item 11 is not part of the Cambridge Depersonalization Scale (CDS; Sierra \& Berrios, 2000). The CDS also gauges dissociative symptoms, and more specifically depersonalization, with an item about autoscopy that describes an experience that is the opposite of DES item 11.

Table 1. Prevalence of the mirror sign (DES item 11) and autoscopy (CDS item 15) in student samples

\begin{tabular}{lccc}
\hline Sample & I DES & II CDS & III DES \\
\hline Size (women) & $213(161)$ & $807(603)$ & $139(106)$ \\
M DES 11/M CDS 15 [95\% CI] & $8.7[6.5,10.9]$ & $1.26[1.2,1.3]$ & $8.0[5.6,10.4]$ \\
$\%>$ cut-off DES (30)/CDS (70) & $28 \%$ & $3.4 \%$ & $23 \%$ \\
Frequency DES 11/CDS 15 & & & \\
$\leq 10$ & $78 \%$ & $93 \%$ & $77 \%$ \\
$\leq 20$ & $85 \%$ & $96 \%$ & $89 \%$ \\
$\leq 30$ & $90 \%$ & $98 \%$ & $92 \%$ \\
$\leq 40$ & $92 \%$ & $99 \%$ & $95 \%$ \\
$\leq 50$ & $95 \%$ & $99 \%$ & $98 \%$ \\
\hline
\end{tabular}

\section{The second sample: Results from the Cambridge Depersonalization Scale}

Item 15 of the Cambridge Depersonalization Scale (CDS) reads as follows: "Whilst fully awake I have 'visions' in which I can see myself outside, as if I were looking at my image in a mirror" (Sierra \& Berrios, 2000), thus referring to autoscopy. The CDS is a self-report scale with 29 symptoms used to screen for the presence of a depersonalization disorder. Autoscopy (item 15) is infrequently reported by patients with depersonalization disorder. Sierra et al. (2005) found an average score of $1.3(S D=2.5)$ in their patient group, which made autoscopy the least mentioned experience of all CDS symptoms. We decided to determine how often an atypical item like this, in which patients who depersonalize are actually supposed to recognize themselves in mirrors, is reported in a non-clinical sample. To investigate this, we recruited a group of 900 Dutch students to participate in research project about food and psychological complaints by means of e-mail and advertisements in local university papers. They completed a digital version of the CDS, along with questionnaires about depressive symptoms and other experiences outside the scope of the present discussion. Participants were offered a small financial compensation for each fully completed questionnaire. In total, 807 questionnaires were completed. The average age of the students was 20 years (range: 18-27 years). 
Following the usual procedure of the CDS, the respondents indicated for each symptom the frequency and duration they had experienced it during the past six months. To indicate frequency they had to choose between: 0 = never; 1 = rarely; 2 = often; 3 = very often; or $4=$ all the time. To indicate duration they had to choose between: 1 = a few seconds; 2 = a few minutes; 3 = a few hours; 4 = about a day; 5 = more than one day; or 6 = more than one week. Cronbach's alphas for duration and frequency were 0.88 and 0.92 , respectively. We were mainly interested in the aforementioned item 15. A large majority of the respondents (93\%) reported that they experienced the symptom rarely or never (see Table 1). On the other hand, 1 in 25 students reported that they experienced autoscopy often (>2).

There are cut-off points for the total scores of the DES and the CDS. A total score above these cut-off points is taken to indicate dissociative symptomatology. In our first sample, $28 \%$ had a total score above the cut-off of the DES. In the second sample, 3.4\% attained a total score above the cut-off of the CDS (see Table 1). The DES thus identified dissociative pathology eight times more often than the CDS. This could possibly be due to the fact that the CDS separates intensity and frequency of symptoms more strictly. Also, the students in the first sample completed the DES in the presence of others, whereas in the second sample the CDS was presented digitally. In any case, these widely differing outcomes offer little support to the notion of a single underlying dissociative trait that manifests itself homogeneously. Apparently, tests for dissociation do not always result in comparable proportions of respondents with scores that surpass the cut-off points.

\section{The third sample: Results from the DES with additional Items}

If neither impending decompensation nor an underlying dissociative trait offers an explanation for the mirror sign, what can explain this? First of all, it is important to note that there is a difference between experiencing the mirror sign and reporting the mirror sign. According to Abély (1930) if psychotic patients experience the mirror sign, they tend to deny it (see also Parnas \& Handest, 2003). Second, if participants in a sample that excludes psychotic patients report that they experience the mirror sign, this could indicate exaggeration or at least careless reporting. Previous studies have explained reports of disturbances in selfrecognition as exaggeration of complaints (Hall \& Poirier, 2001). The underlying reasoning is as follows. The ability to identify oneself in the mirror is based on autobiographical knowledge and passive recognition. Both are rather robust elements, so this ability becomes impaired only after considerable damage to the brain. According to Hall and Porier (2001), except for 
neurological or psychotic patients, it is rather implausible when respondents claim that they are unable to recognize themselves in a mirror.

Does reporting the mirror sign indicate exaggeration? To answer this question, we presented the DES to a third sample of 154 students with an average age of 19.6 years (range: 18-31 years). As in the first sample, this was done during test sessions at the university in which respondents completed several paper questionnaires. However, we added four items to the DES. These were unlikely symptoms that we obtained from the study by Wildman and Wildman (1999): "I have headaches that are so severe my feet hurt"; "The buzzing in my ear keeps switching from the left to the right"; "I notice that the color of objects around me keeps shifting"; and: "I find myself frequently blacking out when I sit down". To assess the frequency of these symptoms. the participants used the same response scale as the DES $(0 \%$ = never; $100 \%$ = always).

Cronbach's alpha for the DES was 0.94. The questionnaires of 15 students had to be excluded because of missing data. The average score on item 11 of the DES was comparable with that in the first sample (see Table 1), and a non-trivial minority (8\%) again stated that experienced this symptom at least $30 \%$ of the time. However, the average scores on unlikely symptoms, such as "having a headache bad enough to make your feet hurt", were at least as high: $11.4(S D=12.4)$. Scores on unlikely symptoms also correlated with the mirror sign: $r=$ 0.41 (Pearson product-moment; $p<0.01$ ). Therefore, the more often respondents reported fake symptoms, the more often they endorsed item 11 . We also calculated what would happen if the 15 respondents (11\%) who exaggerated the most (mean score $>25$ on fake symptoms) were removed from the sample. In that case, only 1 in 20 students (instead of 1 in 10) would report experiencing the mirror sign at least $30 \%$ of the time (first sample). Reporting the mirror sign was thus relatively more prevalent in students who exaggerated the most.

\section{Conclusion}

The mirror sign appeals to our imagination. It is a remarkable symptom, and it may indeed be a phenomenon that accompanies psychotic dysregulation, as Lacan (1966) and his followers asserted. However, we found surprisingly frequent reports of the mirror sign in the three samples of healthy individuals presented here, which strongly suggests that it cannot be regarded as a prodromal sign of psychotic dysregulation. Just as problematic is the 
classification of the mirror sign as a dissociative symptom, which was championed by authors like Steinberg and Schnall (2001). Its symptomatic counterpart, autoscopy, is also presented as a core symptom of dissociation. Researchers in the field of dissociation are rather tolerant towards this extreme symptomatic diversity. For example, both microscopy (perceiving objects smaller than they are in reality) and macroscopy (perceiving objects larger than they are in reality) are included as symptoms in the description of depersonalization (Lipsanen et al., 1999). This demonstrates the poor conceptual demarcation of dissociative symptomatology, which results in dissociative symptoms overlapping with symptom inventories that are used to screen for feigning. The mirror sign is one example, but other items in the DES or the CDS are also worth mentioning in this respect. For example, item 7 of the CDS asks whether the respondent's hands or feet are experienced as becoming larger or smaller. This resembles item 10 on the Structured Inventory of Malingered Symptomatology (SIMS): "I have noticed that my body changes shape even though my weight remains the same," (Van Impelen et al., 2014). Such overlap is worrisome. What does it mean when some people report that they do not recognize themselves in the mirror or have the impression that their feet have grown larger? Is that a dissociative symptom or is it some type of over-reporting (see also: Merckelbach et al., 2015)?

Tests like the DES and the CDS consist entirely of items that are worded in such a way that inattentive and even random responders can attain very high scores that easily exceed the cut-off. Such tests are sensitive to a careless response style (see also Meyer et al., 2013). In addition, neither test includes items that check the validity of the answers, which is a serious omission. Indeed, the DES and the CDS present a long series of eccentric experiences, which may induce a mischievous response style in respondents. This may be regarded as a reactive type of over-reporting (see also Merckelbach, Giesbrecht, Jelicic, \& Smeets, 2010). This variety of response distortions - careless responding or reactive over-reporting - could explain the previously described correlation between reporting the mirror sign (item 11) and the endorsement of fake symptoms.

Our core finding is that scores on tests like the DES and the CDS are sensitive to distortion. Even though a symptom like the mirror sign appears to be based solidly on previous research into psychosis and dissociation, that doesn't make it immune to over-reporting. Several studies have shown that it is easy to get healthy respondents to over-report on tests for dissociation like the DES and the CDS (Giger et al., 2010; Gilbertson et al., 1992). It is not possible to differentiate scores of such simulators from the scores of genuine patients. 
Outside the conditions of the psychology lab, it is not always clear who is a patient and who is a respondent with a tendency to exaggerate. We therefore believe that diagnostic tests as well as research regarding the mirror sign and similar symptoms - should take response distortion into account.

Our analysis, if shown to be correct, could have implications for the question of what dissociative core symptoms are and what can be considered artifacts of self-report. The usual approach to investigate dissociative symptoms is to have participants complete lists like the DES and assume that each reported symptom refers to a latent condition ("dissociation"). The alternative is to present such questionnaires several times during one period and use network analysis, as described by Borsboom and Cramer (2013), to look at symptoms that act as pathological pacesetters and symptoms that occupy a more peripheral position. A good illustration of this type of approach can be found in the study by McNally et al. (2014) into trauma-related symptoms of earthquake victims. Their network analysis showed that indicators of hypervigilance were key players in the symptomatologic cascade, whereas amnesia turned out to be an outlier with no causal significance whatsoever. We believe that this type of approach could be informative about the internal structure of the heterogeneous hodgepodge known as dissociative symptoms, especially if repeated presentation of symptom inventories were combined with instruments that screen for over-reporting (e.g. the SIMS). In that case the more peripheral dissociative symptoms might turn out to be fully accounted for by symptom exaggeration, and have little clinical significance.

We can draw three final conclusions. First, reporting a symptom is not the same as experiencing a symptom. Second, even if a salient symptom has been described at length by generations of experienced clinicians, that does not exclude the possibility that the reports of the symptom were prompted by symptom exaggeration. Third, clinical and basic research into the mirror sign and related symptoms is impossible without checking the validity of symptom reports. For this purpose an instrument like the SIMS can be used, which incidentally includes items that may help to detect inattentive or careless responding. This could yield interesting results especially in replication studies. After all, successful replication has little value if the symptom validity in the target group turns out to be poor. 



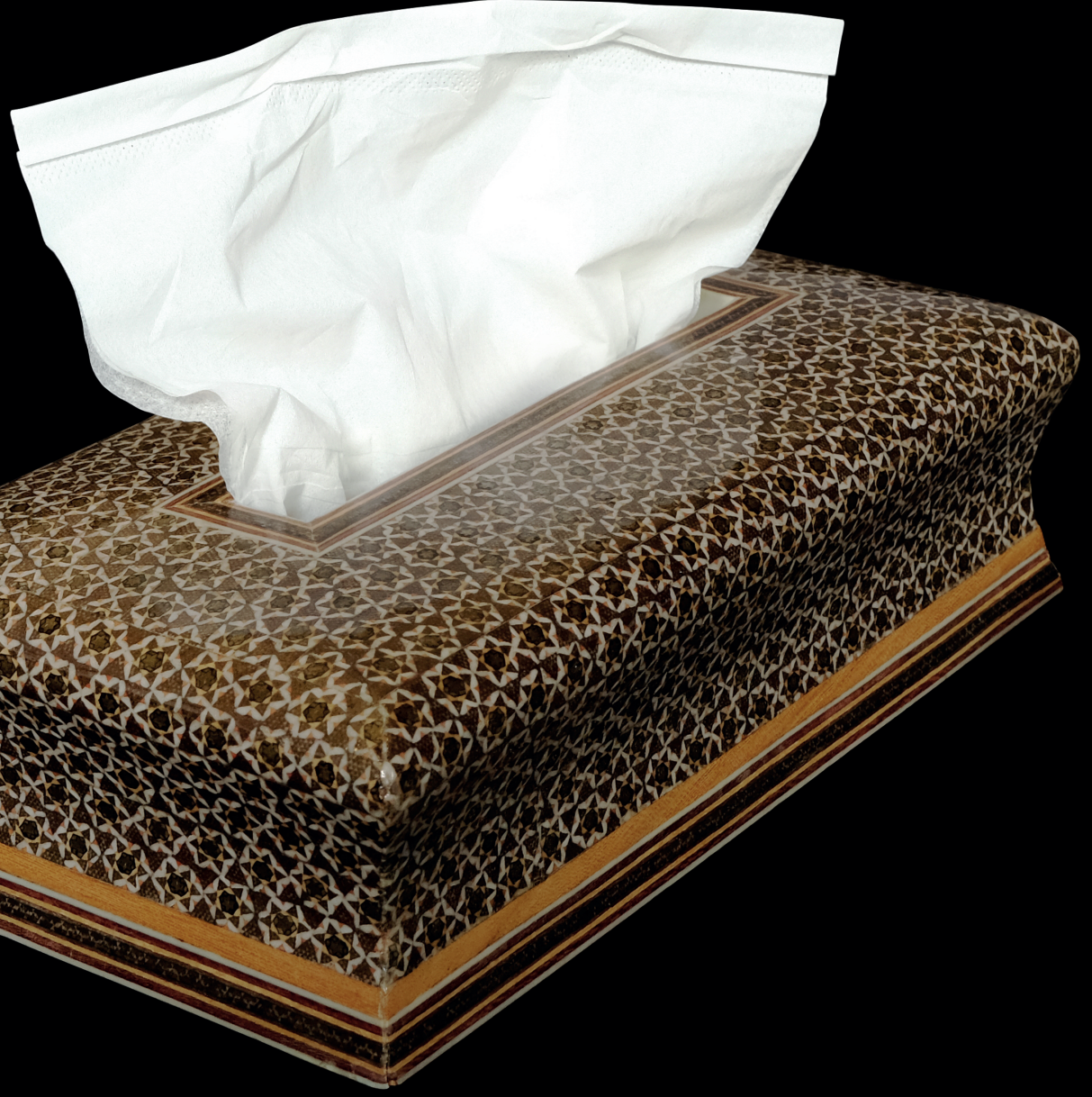




\section{6}

\section{STANDARD SYMPTOM INVENTORIES FOR ASYLUM SEEKERS IN A PSYCHIATRIC HOSPITAL: LIMITED UTILITY DUE TO POOR SYMPTOM VALIDITY}

Van der Heide, D., \& Merckelbach, H. (2017). Standard symptom inventories for asylum seekers in a psychiatric hospital: Limited utility due to poor symptom validity.

Psychological Injury and Law, 10, 358-367. https://doi.org/10.1007/s12207-017-9302-x 


\section{Abstract}

We examined symptom validity in two samples ( $n s=27$ and 35 ) of asylum seekers who had been admitted to a psychiatric facility. Considerable proportions over-endorsed atypical symptoms (63\% and $83 \%$, respectively) and underperformed on a simple forced-choice task requiring the identification of basic emotions ( $41 \%$ and $71 \%$, respectively). Over-endorsement and underperformance were unrelated to Dutch language proficiency but were related to raised scores on standard symptom inventories commonly used to assess psychiatric symptoms of asylum seekers. This pattern of findings casts doubts on attempts to monitor symptom severity and treatment progress in psychiatric asylum seekers without taking symptom validity into account. 


\section{Introduction}

Various groups are known to be at risk for symptom over-reporting. Social security disability claimants (Griffin et al., 1996) and defendants who claim to suffer from psychiatric symptoms are well-studied examples (Rogers et al., 1994). Asylum seekers may also engage in symptom over-reporting. Many Western countries have regulations that grant asylum seekers a permit to stay when there are humanitarian or medical reasons, which may apply when applicants suffer from mental disorders (ECRE, 2016; Meffert et al., 2010). Even after a refugee status has been granted, a psychiatric diagnosis can be advantageous in procedures pertaining to housing, family reunion, and naturalization (Immigration Naturalization Services [IND], 2016; Storm, 2003). Apart from deliberate symptom exaggeration, over-reporting may be the result of an inability to articulate symptoms (i.e., alexithymia; see Söndergaard \& Theorell, 2004), careless responding, confusion/misunderstanding, catastrophizing, negative impression management or feigning in general without the ability to know the conscious or unconscious reasons that might be involved; neither of these categories is mutually exclusive (see for an extensive discussion: Young, 2014). Whatever the reasons for an over-reporting style, the phenomenon in and of itself will likely affect how patients respond to standard clinical instruments administered to them for diagnostic purposes.

Over the past two decades or so, several tests have been developed that can effectively screen for distorted symptom presentation (see for reviews, e.g., Smith, 2008; Sollman \& Berry, 2011; Young, 2014). Some authors (e.g., Weiss \& Rosenfeld, 2017) have referred to these instruments as feigning measures. However, as these tests are often not able to clarify whether distorted symptom presentation is intentional or not, we prefer the more neutral label of Symptom Validity Tests (SVTs). There are two types of SVTs (Egeland et al., 2015): self-report tests consisting of rare symptoms that intend to measure over-endorsement and cognitive tasks that assess underperformance. Both types have proven their added value; in the absence of SVTs, clinicians are poor in determining to what extent patients' symptom presentation is distorted (Dandachi-FitzGerald et al., 2017).

Unfortunately, the extant literature on the cross-cultural stability of SVTs is limited (see, for a review, Nijdam-Jones \& Rosenfeld, 2017). Some SVTs have been translated into other languages and appear to function adequately in ethnic groups for which they were not originally designed (e.g., DuAlba \& Scott, 1993; Geraerts et al., 2009; Montes \& Guyton, 2014; Vilar-Lopez et al., 2007). However, with regard to the culturally highly diverse group of asylum 
seekers there is a paucity of studies (but see Weiss \& Rosenfeld, 2017).

The current study addresses this target group, more specifically asylum seekers in a Dutch psychiatric facility. We reasoned that if SVT outcomes would be intimately associated with the presence of positive incentives (e.g., seeking a permit to stay, needing exemption from the language test required for naturalization) rather than Dutch language proficiency, this would provide initial support for interpreting SVT failures in terms of distorted symptom presentation. In that case, clinicians would be well-advised to interpret data obtained with standard clinical instruments with caution, keeping at the same time in mind that the presence of positive incentives per se does not necessarily imply that symptom over-reporting reflects feigning.

In mental health facilities that specialize in asylum seekers, one limitation associated with SVTs - and, indeed, all psycho-diagnostic tests and structured interviews for that matter - is that optimal translations of instruments for culturally highly diverse groups are often not available. Thus, it is standing practice that professional interpreters are involved in on the spot translations of test items that are read out by clinicians during the diagnostic evaluation. This approach may compromise validity due to subtleties in items and answers that are easily lost during live interpretations (Bot, 2005). Unlike a clinical interview, where follow-up questions can help clarify responses or make sure that the patient understood the items, standardized instruments are intended to be administered in a fixed format without any interruptions by the clinician. On the spot translations may result in data that cannot be compared to existing normative data. This raises the question whether deviant scoring on SVTs can be attributed to this suboptimal method of presentation. In an attempt to control at least partially for this significant confounder, we reasoned that one would expect asylum seekers with good Dutch proficiency to pass SVTs more often than those with limited proficiency, because the first group depends less on the translations of the interpreter. That is, compared with their low proficiency counterparts, the high proficiency group would be better able to understand the original text read out aloud by the staff member presenting the test items. It stands to reason that this group is also more acculturated to Dutch society and has more experience in taking Western-style tests (which are common during Dutch language courses).

In their pilot study, Van der Heide and Merckelbach (2016) administered SVTs to asylum seekers who were treated in a psychiatric facility. Specifically, with help of professional translators, the authors administered items taken from the Structured Inventory of Malingered Symptomatology (SIMS; Smith \& Burger, 1997) and a forced-choice task modeled after Morel's 
Emotional Numbing Test (MENT; Morel, 1998). Both symptom over-reporting on SIMS items and underperformance on the forced-choice task occurred on a nontrivial scale (i.e., rates between $41 \%$ and $87 \%$ ) and were not related to Dutch language proficiency. However, the various subsamples in this study were small and standard self-report instruments to index psychiatric symptoms were not included. In the current study, we explored whether deviant performance on SVTs goes hand in hand with escalated symptom endorsement on standard diagnostic instruments among refugee patients. Specifically, we were interested in scores on the Dissociative Experiences Scale (DES; Bernstein \& Putnam, 1986) and the Harvard Trauma Questionnaire (HTQ; Mollica et al., 1992). Both DES and HTQ are widely used in refugee communities throughout the world (Carlson \& Rosser-Hogan, 1993; Favaro et al., 1999; Mollica et al., 1992; Shoeb et al., 2007). In the first sample, we related SVT outcomes to the DES, while in the second sample, SVT outcomes were related to the HTQ. In both samples, we took Dutch proficiency and the presence of incentives into account.

\section{Method}

\section{Participants}

We recruited two inpatient samples from a psychiatric facility with 32 beds for inpatient treatment of asylum seekers. This facility is part of a general psychiatric hospital in the Netherlands and serves as a national referral center for non-forensic refugee mental health. As a rule, patients are referred after usual outpatient treatments have been ineffective. Most of their symptoms can be classified as part of a posttraumatic stress disorder (PTSD), but many patients also present affective, dissociative or psychotic symptoms secondary to trauma.

Both samples are here referred to as 'asylum seekers', but actually included asylum seekers whose procedure was still ongoing as well as former asylum seekers with a refugee status and a residence permit. All inpatients present in the clinic during the time frame of the study were included, except for a few patients who refused informed consent or were unable to give such consent because of severe disorientation. During the time frame of the study, the average length of admission varied between 6 and 9 months. Data in the first sample were obtained during a two-month period in 2009; data in the second sample were obtained during a five-month period in 2010.

Sample 1. The first sample included 27 asylum seekers (70\% men). Mean age was 
34.5 years (range: 20 - 51 years); mean level of education 10.7 years $(S D=4.8)$. All of them were given the DES items (see below). Eleven patients (41\%) came from Africa, eight (30\%) from the Middle East, four (15\%) from the Far East, three (11\%) from the former USSR, and one (4\%) from former Yugoslavia.

Ten asylum seekers (37\%) had a low level of proficiency in Dutch, the language of the host country (see below). Nine (33\%) had an intermediate level and eight (30\%) were advanced students. Six respondents (22\%) had a negative incentive, nine (33\%) had mixed incentives, and twelve (44\%) a positive incentive (see below).

Sample 2. The second sample consisted of 35 patients ( $80 \%$ men), who were interviewed with the HTQ (see below); besides asylum seekers, this sample included one regular nonWestern migrant with a history of severe psychological trauma.

Mean age was 27 years (range: 15 - 55 years); mean level of education was 6.4 years (SD = 5.2); five patients (14\%) originated from the former USSR, twenty two (63\%) from Africa, six (17\%) from the Middle East, and two (6\%) from the Far East.

Eighteen (51\%) patients had a poor proficiency in Dutch; twelve (34\%) an intermediate proficiency, and only one (3\%) was classified as an advanced student. In four cases (11\%) the assessment was missing. Five patients (14\%) had a negative incentive, another five (14\%) mixed incentives, and twenty-five (71\%) a positive incentive.

\section{Materials}

The Dissociative Experiences Scale (sample 1). The Dissociative Experiences Scale (DES; Bernstein \& Putnam, 1986) is a self-report scale that requires participants to indicate on $100 \mathrm{~mm}$ visual analogue scales (anchors: 0 = never; 100 = always) to what extent they experience 28 dissociative experiences in daily life (amnesia, depersonalization, absorption). A typical sample item is: "Some people find that sometimes they are listening to someone talk and they suddenly realize that they did not hear part or all of what was just said. Mark the line to show what percentage of time this happens to you."

Items of the Dutch DES (Boon \& Draijer, 1993) were presented to patients by a sixth year medical intern with the help of a professional interpreter; when patients were unable to indicate a percentage, they were encouraged to use verbal quantifiers (e.g., "never", "occasionally", "fairly often", "very often", and "always"; Wright \& Loftus, 1999). Scores were summed across items to obtain a total DES score (range: 0-100). Values above 30 (Putnam et al., 1996) are considered to be indicative of clinically raised dissociation levels. 
The Harvard Trauma Questionnaire (sample 2). The Harvard Trauma Questionnaire (HTQ; Mollica et al., 1992) is a self-inventory of traumatic experiences. In the current study, we used the Dutch version (Kleijn \& Mook, 1999). It has four parts: part I is an inventory of past traumatic events (17 items) with four possible responses for each event: "Experienced," "Witnessed," "Heard about it," or "No." Illustrative items are: "lack of food or water", "torture" or "rape". Part II asks respondents for a subjective description of the most traumatic event(s) they experienced. Part III is an inventory of incidents that may have caused traumatic brain injury (drowning, suffocation, blows to the head, and subsequent loss of consciousness). Part IV is an inventory of 30 symptoms. Sixteen of these are derived from the DSM-III-R criteria for PTSD (part IV-a); the remaining fourteen items were devised to target symptoms associated with refugees" traumatic life events (part IV-b). Illustrative items are "feeling as if you are going crazy" and "feeling that someone you trusted betrayed you". Items of Part IV are rated on a 4-point scale ("Not at all," "A little," "Quite a bit," and "Extremely", which are scored 1, 2, 3 and 4 , respectively). The total score of the HTQ is the sum of the ratings of part IV symptoms divided by 30 (range: 1-4). Items of the HTQ were presented to patients by a fourth year psychology student with help of a professional interpreter who also translated the responses.

Implausible symptoms (sample 1 and 2). We used all the items of the Structured Inventory of Malingered Symptomatology (SIMS; Smith \& Burger, 1997) to index over-reporting. In its original form, the SIMS is a self-report instrument that consists of 75 true-false items that describe atypical and extreme symptoms (e.g., "There is a constant ringing in my ear"; "The voices that I hear, have never stopped since they began"). There are five subscales, each containing 15 items, which address the following conditions: amnesia, neurologic impairment, psychosis, affective disorders, and low intelligence. After recoding some items, endorsed symptoms are summed to obtain a total SIMS score (range: 0-75), with higher scores indicating more symptom endorsement. Van Impelen et al. (2014) summarize psychometric data indicating that the internal consistency of the SIMS is satisfactory (with Cronbach's alpha's ranging from 0.80-0.96), its test-retest stability sufficient $\left(r^{\prime} s=0.72-0.97\right)$, and its ability to discriminate between symptom exaggeration and honest responding fairly effective (with sensitivities varying between 75\% and 100\%). Nijdam-Jones and Rosenfeld (2017) concluded in their recent review of studies on cross-cultural feigning assessment that of all self-report SVTs included in their review, the SIMS had the highest overall classification accuracy. Previous studies recommended a cutoff of 16 to screening for symptom over-reporting (Merckelbach \& Smith, 2003). This cutoff is problematic when the SIMS is employed as a measure to detect individual feigners. 
This, however, was not our goal. We employed the translated SIMS items as a global screening measure for over-reporting and as the extensive review of Van Impelen et al. (2014) shows, the SIMS is sensitive to differential prevalence in that context.

For the purpose of the current study, items 11, 14, and 60 of the Dutch version of the SIMS (Merckelbach et al., 2001) were adapted. Items 11 and 14 refer to Western oriented geography and after consultation with professional interpreters, they were reformulated. For example, item 11- "The capital of Italy is Hungary" - was changed to "The capital of Turkey is Azerbaijan" for asylum seekers from Armenia. The original item 60 pertained to the queen of Holland in the Dutch version and was rephrased as follows: "The prophet of Allah is called Mohammed" (for patients with a Muslim background) and "The mother of Jesus is called Mary" (for patients with a Christian background). The SIMS was presented to the asylum seekers by a psychiatrist with the help of a professional interpreter, who also back-translated the yes- or no-answers.

Forced-choice task (Sample 1 and 2). To screen for underperformance, we employed a forced-choice task involving the identification of basic emotional expressions modeled after the Morel Emotional Numbing Test (MENT; Morel, 1998). We included this task because it has low verbal mediation (see also Benuto et al., 2014; Erdodi, Nussbaum et al., 2017). Thus, compared with the DES, HTQ or SIMS items, the forced-choice task required only minimal effort to translate test items, which makes it an interesting instrument for cross-linguistic contexts.

Our version was adapted from Geraerts et al. (2007) and consisted of 20 colored slides of 10 facial expressions posed by a man and a woman. Their expressions reflected happiness, frustration, sadness, anger, fear, calmness, surprise, shyness, confusion, and sleepiness. The slides were presented on a computer screen $(30 \times 38 \mathrm{~cm})$. Patients were instructed to identify the emotion that best matched the expression of the face. In a first series of 20 trials, patients had to indicate which of two words (e.g., "happy" versus "surprised") best described the facial expression in the picture. In a second run of 20 trials, patients viewed two slides with different expressions at the same time, and only one emotion word. They had to identify the expression that best matched the word. In a final run of 20 trials, patients were shown two slides and two words in each slide that had to be connected in the correct way. Emotional labels used in the test were translated and back-translated into several languages. The forcedchoice task was administered by a psychiatrist. However, as some asylum seekers reported to be unable to read or write and some translations were not available in the native language of the asylum seeker (e.g., a Russian translation for asylum seekers from former Soviet Republics), 
a professional interpreter was present during the test to assist with the instructions and the key verbal labels when necessary.

Before the test, patients were told that emotional numbness is a prominent symptom of trauma-related problems and that this may cause people to have difficulties with the recognition of facial expressions. The rationale behind this instruction is that individuals who want to overstate trauma-related symptoms will produce more errors. Errors were summed across the three runs. Morel (1998) recommended a cutting score of nine errors on the MENT, with scores above this level raising the suspicion of underperformance. In a sample of Croatian war veterans elevated error levels on our version of the forced-choice task was found to be effective in differentiating between treatment-seeking and compensation-seeking veterans (sensitivity: 92\%; specificity 96\%; Geraerts et al., 2009).

\section{Procedure}

Before test items were administered, patients were informed that some tests would be used to assess the validity of symptom reporting in their target group. We explained that if results would indicate poor validity, this would mean that standard symptom inventories do not provide useful information and that diagnosis would have to be based on additional interviews and observations. Only patients who gave informed consent for anonymous use of their data for scientific purposes were included. The study was approved by the Central Committee on Research Involving Human Subjects (CCMO).

The SVTS - SIMS items and the forced-choice task- were presented in counterbalanced order. DES or HTQ items were administered during a separate session, either before or after SVT results had been obtained.

Proficiency in Dutch was independently assessed by certified Dutch language teachers of the hospital. All teachers were specifically trained to instruct students with little or no knowledge of the Dutch language and used the European reference scale (Meijer \& Noijons, 2008) that has been developed to differentiate between different levels of proficiency. This way, we were able to assign asylum seekers to three groups: those with poor, intermediate, and good proficiency. The language teachers had no access to information about the performance of the patients on the various tests.

Social workers of the hospital independently evaluated incentive levels of patients on the basis of file information. Relevant information was in the files of the patients through contacts between social workers and legal representatives or lawyers of the patients. As a rule, patients 
were aware of the presence of such information as Dutch law requires informed consent for legal steps such as application for asylum for medical reasons or child custody procedures. Thus, social workers could differentiate between the presence of positive incentives, the absence of incentives, and the presence of negative incentives. They did this by adding one point for each condition that potentially might promote symptom over-reporting, such as: 1 . An asylum procedure still in progress; 2. A temporary refugee status issued for medical reasons; 3 . Any other current procedure requiring a medical report indicating medical necessity, urgency or exemption (e.g., request for family reunion while the patient is not able to generate the necessary income demanded by Dutch law; a request for urgent change of housing or special housing arrangements; a request to be exempted from the demand to pass the language test in the naturalization procedure). For each condition discouraging symptom over-reporting they subtracted a point. Such circumstances would be: 1 . Compulsory admission; 2. Any current procedure requiring a medical report indicating improved functioning or decreased need for medical treatment or scrutiny (e.g., a child custody procedure, a request for voluntary repatriation). Patients with one point or more were considered to have a positive incentive, and patients with minus one point or less were considered to have a negative incentive. Social workers evaluating incentive levels had no knowledge about the test outcomes.

Staff members who presented the standard symptom inventories (DES or HTQ) and the SVTs were not aware of the information obtained by the social workers and the Dutch language teachers. However, as staff members they were involved in the treatment of the patients and had access to their medical files. So, in this respect, blinding was not complete.

\section{Data analysis}

We used descriptive statistics to evaluate scores on standard symptom inventories and SVTs. Depending on whether data were skewed or evenly distributed, we employed t-tests, one-way Analyses of Variance (ANOVA's) or Kruskal-Wallis and Mann-Whitney U tests to compare groups that differed in language proficiency and incentives with regard to their symptom reports, endorsement of implausible symptoms, and errors on the forced-choice task. 


\section{Results}

\section{Sample 1}

Dissociative symptoms (DES). There were no missing data and data were not normally distributed (Shapiro-Wilks $p<0.05)$. Cronbach's alpha was 0.97. The mean DES score was $22.6,95 \% \mathrm{Cl}[14.5,30.7]$, with a range of $0-70.4$. Ten patients (37\%) scored $\geq 30$. Low, intermediate, and high proficiency groups had mean DES scores of $25.1(S D=22.7)$, $17.4(S D=22.5)$, and $25.5(S D=20.5)$; a Kruskal-Wallis test was non-significant: $\chi^{2}(2)=1.2$, $p=0.54$. A Kruskal-Wallis test performed on DES scores as a function of incentives did attain significance: $\chi^{2}(2)=14.9, p<0.01, \eta^{2}=0.43$. Further details are given in table 1 .

Implausible symptoms. There were no missing data and data were not normally distributed (Shapiro-Wilks $p<0.05$ ). Cronbach's alpha for the full list of implausible items was 0.96. Cronbach's alpha's for subscales ranged from 0.74 (affective disorders) to 0.91 (amnesia). The mean endorsement rate of implausible symptoms was $27.0,95 \% \mathrm{Cl}[20.4,33.6]$ with a range of 5-59. Seventeen patients (63\%) scored above the cutoff (16).

Low, intermediate, and high proficiency groups had mean scores of 33.4 (SD = 19.4), 20.4 (SD $=14.0)$, and $26.5(S D=17.6)$, respectively; a Kruskal-Wallis test remained non-significant: $\chi^{2}(2)$ $=2.6, p=0.24$. A Kruskal-Wallis test on implausible symptoms endorsement as a function of incentives was significant: $\chi^{2}(2)=18.2, p<0.01, \eta^{2}=0.54$ (see table 1 ).

Forced-choice task. There were no missing data in this data set. The data were not normally distributed (Shapiro-Wilks $p<0.05$ ). Cronbach's alpha for the forced-choice task was 0.95. The mean error score was $12.4,95 \% \mathrm{Cl}[7.9,16.9]$ with a range of 0 - 42; eleven patients (41\%) scored above the cutoff ( 9 errors); two patients (7\%) failed on more than half of the items of the forced-choice task.

Low, intermediate, and high level subgroups had mean error scores of 17.7 (SD = 14.4), $10.7(S D=10.0)$, and $7.8(S D=7.5)$, respectively. A Kruskal-Wallis test remained nonsignificant: $\chi^{2}(2)=3.3, p=0.19$. Again, a Kruskal-Wallis test performed on error scores of the three incentive groups was significant: $\chi^{2}(2)=12.1, p<0.01, \eta^{2}=0.33$ (see table 1 ). 
Table 1. Mean DES scores, implausible symptoms, and forced-choice task errors of patients with relatively more negative or more positive incentives. Those with a "neutral" incentive status are shown in the middle. 95\% confidence intervals [Cl's] are also shown.

\begin{tabular}{|c|c|c|c|c|}
\hline & $\begin{array}{l}\text { Negative } \\
(n=6)\end{array}$ & $\begin{array}{l}\text { Neutral } \\
(n=9)\end{array}$ & $\begin{array}{l}\text { Positive } \\
(n=12)\end{array}$ & $\chi^{2}(2)$ \\
\hline DES & $\begin{array}{l}1.1(1.2) \\
{[0.7,1.6]}\end{array}$ & $\begin{array}{l}16.7(18.0) \\
{[9.9,23.5]}\end{array}$ & $\begin{array}{l}37.8(18.0) \\
{[31.0,44.6]}\end{array}$ & $14.9^{* *}$ \\
\hline Implausible symptoms & $\begin{array}{l}11.7(4.4) \\
{[10.0,13.4]}\end{array}$ & $\begin{array}{l}15.2(10.2) \\
{[11.4,19.0]}\end{array}$ & $\begin{array}{l}43.4(9.2) \\
{[39.9,46.9]}\end{array}$ & $18.2^{* *}$ \\
\hline Errors forced-choice task & $\begin{array}{l}6.8(3.3) \\
{[5.6,8.0]}\end{array}$ & $\begin{array}{l}4.8(4.3) \\
{[3.2,6.4]}\end{array}$ & $\begin{array}{l}21.2(12.7) \\
{[16.3,25.9]}\end{array}$ & $12.1^{* *}$ \\
\hline
\end{tabular}

${ }^{*} p<0.05 ;{ }^{* *} p<0.01$

Correlations between measures. We computed Spearman rank correlations between DES scores, implausible symptom endorsement, and errors on the forced-choice task. Forcedchoice errors correlated at $r h o=0.74(p<0.01)$ with implausible symptom endorsement, suggesting that both tests tap into symptom over-reporting. Also, both forced choice errors and implausible symptoms correlated significantly with DES symptoms: rho $=0.41(p<0.05)$ and $r h o=0.79(p<0.01)$, respectively.

\section{Sample 2}

HTQ. There were no missing data for the HTQ. The number of traumatic events that patients rated as 'experienced' in part I ('self-reported traumatic events'), the number of events suggestive of traumatic brain injury (part III), and scores on part IV-a (PTSD symptoms) were not normally distributed (all Shapiro-Wilks p's < 0.05). The HTQ scores (part IV, all 30 items) were normally distributed (Shapiro-Wilks $p=0.12$ ). Cronbach alpha's for part I, III, IV-a and IV were $0.86,0.78,0.85$, and 0.92 , respectively. The mean number of self-reported traumatic events was $10.4,95 \% \mathrm{Cl}[9.0,11.8]$ with a range of 1 - 16; the mean number of events suggestive of traumatic brain injury was $3.1,95 \% \mathrm{Cl}[2.4,3.9]$ with a range of $1-6$, the mean score on part IVa (16 PTSD items) was $2.5,95 \% \mathrm{Cl}[2.3,2.7]$ with a range of $1.2-3.5$, and the mean $\mathrm{HTQ}$ score (averaged over 30 items) was $2.4,95 \% \mathrm{Cl}[2.2,2.6]$ with a range of $1.1-3.5$. For the 16 PTSD items, 22 patients (63\%) had a score $>2.5$, and for all 30 items of part IV 21 patients $(60 \%)$ had a score $>2.5$. 
To test the effects of Dutch proficiency, levels were recoded as "poor proficiency" (level A0 according to the European reference scale, $n=18$ ) and "sufficient proficiency" (level A1 and above, $n=13)$. Mean group scores were $9.9(S D=4.6)$ and $10.3(S D=4.0)$, respectively, for self-reported traumatic events; $2.8(S D=2.2)$ and $3.5(S D=2.4)$, respectively, for self-reported traumatic brain injury incidents; $2.5(S D=0.6)$ and $2.6(S D=0.6)$ for PTSD symptoms of part IV-a. Mann-Whitney $U$ tests showed that the two proficiency groups did not differ in selfreported traumatic events, events suggestive of traumatic brain injury, and PTSD symptoms $: U=115.0, p=0.94 ; U=96.5, p=0.40 ;$ and $U=106.5, p=0.67$ respectively. Neither did the two proficiency groups differ in overall HTQ scores, $t(29)<1.0, p=0.48$, means being 2.4 (SD $=0.7)$ and 2.5 (SD = 0.6) for the poor and sufficient groups, respectively. On the other hand, when the sample was broken down into different incentive subgroups significant differences emerged. This was true for self-reported traumatic events, brain injury incidents, and PTSD symptoms such that those with relatively more negative incentives reported lower numbers in all three categories: $\chi^{2}(2)=8.8, p<0.01, \eta^{2}=0.18 ; \chi^{2}(2)=12.2, p<0.01, \eta^{2}=0.27$; and $\chi^{2}(2)$ $=13.2, p<0.01, \eta 2=0.38$, respectively. A one-way ANOVA indicated that incentive groups also differed with regard to overall HTQ scores: $F(2,32)=18.7, p<0.001, \eta^{2}=0.21$. Further details are given in table 2 .

Implausible symptoms. A total of 20 items was left unanswered in all 35 interviews, which is less than $1 \%$. These missing data were treated as non-endorsements. The data were not normally distributed (Shapiro-Wilks $p<0.05$ ). Cronbach's alpha for the full scale of implausible symptoms was 0.95 and ranged from 0.69 (affective disorders) to 0.90 (amnesia) for the subscales. The mean score was 38.2, 95\% Cl [33.3, 43.4], ranging from 4 to 59. Twentynine patients (83\%) scored above the cutoff. Mann-Whitney $U$ tests showed no significant differences for proficiency levels: $U=109.0, p=0.75$. Mean endorsement rates for poor and sufficient proficiency groups were $36.7(S D=19.5)$ and $40.4(S D=13.2)$, respectively. A KruskalWallis test indicated that implausible symptom levels were significantly higher in the group with positive incentives: $\chi^{2}(2)=13.5, p<0.01, \eta^{2}=0.30$. Further details are given in table 2 .

Forced-choice task. There were no missing data for the forced-choice task and error scores were not normally distributed (Shapiro-Wilks $p<0.05$ ). Cronbach's alpha was 0.94. Mean number of errors was 21.1, 95\% Cl [16.4, 25.7], ranging from 1 to 50. Twenty-five patients (71\%) scored above the cut-off; eleven (31\%) failed on more than half of the items. Differentiating between proficiency levels did not result in any significant group differences in error rates: $U=99.0, p=0.47$. Mean errors for poor and sufficient proficiency groups were 
$20.2(S D=14.5)$ and $24.8(S D=12.7)$, respectively. A Kruskal-Wallis test showed that patients with incentives made more errors on this task: $\chi^{2}(2)=10.4, p<0.01, \eta^{2}=0.22$. Further details are given table 2 .

Table 2. Mean number of self-reported traumatic events (HTQ part I), brain injury incidents (HTQ part III), PTSD symptoms (HTQ part IV-a), full HTQ part IV symptoms, implausible symptoms, and forced choice task errors of patients with relatively more negative or more positive incentives. Those with a "neutral" incentive status are shown in the middle. 95\% confidence intervals [Cl's] are also shown.

\begin{tabular}{lcccc}
\hline & $\begin{array}{c}\text { Negative } \\
(n=5)\end{array}$ & $\begin{array}{c}\text { Neutral } \\
(n=5)\end{array}$ & $\begin{array}{c}\text { Positive } \\
(n=25)\end{array}$ & $\chi^{2}(2) ; F(2,32)$ \\
\hline $\begin{array}{l}\text { HTQ part I } \\
\text { (traumatic events) }\end{array}$ & $\begin{array}{c}4.0(4.0) \\
{[2.7,5.3]}\end{array}$ & $\begin{array}{c}11.6(2.4) \\
{[10.8,12.4]}\end{array}$ & $\begin{array}{c}11.4(3.4) \\
{[10.3,12.5]}\end{array}$ & $8.8^{* *}$ \\
HTQ part III & $0.4(0.9)$ & $2.0(2.0)$ & $3.9(1.9)$ & $12.2^{* *}$ \\
(brain injury) & {$[0.1,0.7]$} & {$[1.3,2.7]$} & {$[3.3,4.5]$} & \\
HTQ part IVa & $1.5(0.3)$ & $2.3(0.6)$ & $2.7(0.4)$ & $13.2^{* *}$ \\
(PTSD symptoms) & {$[1.4,1.6]$} & {$[2.1,2.5]$} & {$[2.6,2.8]$} & \\
& $1.3(0.2)$ & $2.3(0.6)$ & $2.6(0.4)$ & $18.7^{* *}$ \\
HTQ part IV & {$[1.2,1.4]$} & {$[2.1,2.5]$} & {$[2.5,2.7]$} & \\
(all symptoms) & $12.0(6.0)$ & $26.6(19.7)$ & $43.4(11.5)$ & $13.5^{* *}$ \\
& {$[10.0,14.0]$} & {$[20.1,33.1]$} & {$[39.6,47.2]$} & \\
Implausible symptoms & & $11.8(9.6)$ & $25.5(13.0)$ & $10.4^{* *}$ \\
& $8.0(5.3)$ & {$[8.6,15.0]$} & {$[21.2,29.8]$} & \\
Errors forced-choice & {$[6.2,9.8]$} & & & \\
task & & & & \\
\hline
\end{tabular}

${ }^{*} p<0.05 ;{ }^{* *} p<0.01$

Correlations between measures (full sample). Endorsement of implausible symptoms correlated at $r h o=0.59$ with errors on the forced-choice task $(p<0.01)$, suggesting that both measures tap into a common conceptual domain of distorted symptom presentation. The HTQ symptoms correlated at $r h o=0.43$ with endorsement rates of implausible symptoms $(p$ $<0.01$ ). The correlation between HTQ and errors on the forced choice task remained nonsignificant ( $r h o=0.31, p=0.07)$. 


\section{Discussion}

Our main findings can be summarized as follows. First, considerable proportions in samples 1 and 2 over-endorsed implausible symptoms (63\% and 83\%, respectively) and underperformed on a simple forced-choice task ( $41 \%$ and $71 \%$, respectively). Meanwhile, these proportions should not be taken as precise estimates of the base rate of feigning among refugees or asylum seekers. After all, these proportions are based on SVTs that were administered in a suboptimal way and in a context in which cutoffs might not have enough accuracy. What is more informative are the mean endorsement rates for implausible symptoms and the mean error rates on the forced-choice task in the positive incentive groups. These means are way beyond the cutoffs and are suggestive of symptom over-reporting. Second, replicating earlier findings in similar samples (van der Heide \& Merckelbach, 2016), over-endorsement and underperformance were unrelated to language proficiency. While controlling for language proficiency is not likely to cover the confounding effects of live interpretation of test items entirely, this pattern does contradict the idea that deviant scoring on SVTs in this group is merely an artifact of poor language proficiency and/or the involvement of interpreters.

Third, in both samples, over-endorsement and underperformance were significantly associated with incentives, such that a stronger presence of positive incentives went hand in hand with higher levels of distorted symptom presentation. The intimate connection between problematic symptom validity and the presence of positive incentives is a recurrent theme in neuropsychological studies on litigating or compensation-seeking patients (e.g., Bianchini et al., 2006). Fourth, overall, over-endorsement and underperformance was associated with raised scores on standard clinical instruments (i.e., DES and HTQ). Studies in other settings (e.g., psychiatric outpatients: Dandachi-FitzGerald et al., 2011; veterans: Wisdom et al., 2014) also found that deviant scoring on SVTS correlates with heightened scores on standard diagnostic tests.

Our finding of non-trivial proportions of psychiatric asylum seekers who over-report symptoms and who underperform parallels earlier findings. Studying psychiatric asylum seekers in the same facility, Van der Heide and Merckelbach (2016) observed that $87 \%$ of patients endorsed more items than the cuf-off ( $>16$ ) of the SIMS and that 58\% of the patients made more errors than the cut-off (>9) of the forced-choice task. In that study too, problematic symptom validity could not be explained by poor language proficiency, but was linked to the presence of positive incentives. It is important to emphasize that such findings do not rule 
out the possibility that different cultural attitudes towards test taking shape performance on SVTs. In fact, there is every reason to suspect that people from different cultures differ in their test behavior (Ardilla, 2005). We did not look into this complex issue (see for a review, Nijdam-Jones \& Rosenfeld, 2017).

Another important point to consider is that our study was conducted in a highly specialized, clinical setting for therapy-resistant cases. Thus, our findings cannot be generalized to asylum seekers in general and they certainly are silent about the prevalence of feigning among refugees and asylum seekers. Although positive incentives were associated with failures on two different SVTs and patients, as a rule, will have been aware of their positive incentives, this constellation is not enough to conclude anything about possible feigning. Apart from the fact that our sub-optimally administered SVTs were merely used as screening instruments, the differential prevalence design (Rogers, 2008) of our study does not permit any definite classification. In fact, recent research suggests that the use of SVTs in traumaexposed African immigrants may lead to high rates of false positive classifications of feigning (Weiss \& Rosenfeld, 2017).

Our finding of a connection between SVT failure and heightened scores on standard clinical instruments is important. It raises the question how much trust can be placed in the outcomes of these instruments in the current setting. Several authors have pointed out that SVT failure may render diagnostic information obtained with standard clinical instrument largely noninterpretable (e.g., Fox, 2011). Ignoring poor symptom validity might be even dangerous, particularly when clinicians base their treatment interventions on self-reported symptom information that is so distorted that it obscures the real underlying disorder and, consequently, affects treatment decisions (Bush et al., 2005; Institute of Medicine, 2015).

Our findings do cast doubts on attempts to monitor symptom severity in asylum seekers with self-report instruments such as the DES and the HTQ in the absence of any checks on symptom validity. Note that the overall DES and the HTQ scores that we obtained were similar to those reported in other studies. The mean DES score in the present study (i.e., 22.6, $S D=21.5)$ comes close to the average DES score of $20.0(S D=18.1)$ found in a sample of Dutch psychiatric inpatients (Friedl \& Draijer, 2000) and to that of 18.5 (SD =10.8) observed in refugees from former Yugoslavia in an Italian refugee camp (Favaro et al., 1999). Carlson and Rosser-Hogan (1993) even reported a mean DES score of 37.1 (SD = 16.1) in Cambodian refugees living in the United States. Similarly, for the HTQ, van Dijk et al. (1999) observed in their sample of patients admitted to a psychiatric facility for asylum seekers a mean number 
of self-reported traumatic events of $8.9(S D=5.0)$ and a mean $\mathrm{HTQ}$ of $2.4(S D=0.8)$; Kleijn et al. (1998) found in their sample of patients referred to a psychiatric facility for asylum seekers (both clinical and outpatient) a mean number of self-reported traumatic events of 10.3 (SD = 4.3). All these scores are well in line with what we observed: a mean number of self-reported traumatic events of $10.4(S D=4.2)$ and a mean HTQ score of $2.4(S D=0.6)$. Without the red flags that the SVTs provided us with, the outcomes of the present study could simply have been construed as replicating earlier epidemiological studies in refugee populations. Our point is that more precise estimates as to the prevalence and severity of psychopathology is possible when one corrects for the distorting influence of symptom over-reporting by taking SVT failures into account (see also Merckelbach et al., 2014).

Four important limitations of the current study deserve some comment. First, in our study, proficiency in Dutch language was employed not as a proxy for acculturation, but rather as a measure to control for the potential confounding effects of suboptimal test presentation. Live interpretation of the items of standard instruments may serve to invalidate the results obtained because of the alteration in the standardized procedure in testing. Our reliance on interpreters was inspired by the Dutch practice of using interpreters for clinical interviewing and testing, the underlying idea being that with their help in translating standard instruments for which no official back-translated version is available (e.g., in Armenian), it is- within limitspossible to arrive at a diagnosis. The implication of our findings for this suboptimal approach to diagnosis, is that it is wise to take SVT results into account. Second, in our study, social workers inspected patient files for verifiable positive and negative incentives. More subtle motives for over-reporting symptoms (e.g., earlier experiences of care being denied) or under-reporting were not included in their assessment. The latter might be particularly relevant given the lowered scores on standard symptom inventories (e.g., the DES) in the minority of patients with negative incentives. This might reflect denial of symptoms, which is a topic that warrants further research.

Third, how incentives precisely play a role in symptom misrepresentation can only be understood if one would administer the SVT items employed in the current study to other groups as well, e.g., non-clinical samples of asylum seekers. Fourth, our samples were relatively small and our design was cross-sectional in nature. It would be informative to monitor the symptomatic course over time of psychiatric asylum seekers who fail and who pass SVTs. Such longitudinal set-up could elucidate how adverse living conditions may foster problematic symptom validity. 


\section{Conclusions}

Over-endorsement of SIMS symptoms and excessive errors on a simple forced-choice task were related to heightened symptom levels on standard clinical instruments that are commonly used in the diagnostic assessment of psychiatric asylum seekers. Furthermore, for all instruments, elevated symptom scores were related to positive incentives rather than language proficiency. This pattern of findings casts doubts on attempts to monitor symptom severity and treatment progress in psychiatric asylum seekers without taking the validity of symptom reports into account.

While our study highlights the problems in relying on self-report inventories to monitor treatment progress, the data of this study cannot be used to determine the frequency of feigning in this sample. Its findings cannot be generalized to asylum seekers in general and they certainly are no indication of the prevalence of feigning among refugees and asylum seekers. Even though the presence of positive incentives was the most prominent correlate of problematic symptom reports in our samples of asylum seekers, there is little doubt that asylum seekers in general are a highly vulnerable group with high rates of psychopathology, notably trauma-related psychopathology (Reko et al., 2015). Our results do raise the possibility, however, that in a considerable subgroup of psychiatric asylum seekers the various medicolegal procedures may have contributed to poor symptom validity to an extent that compromises diagnostic decision making. 



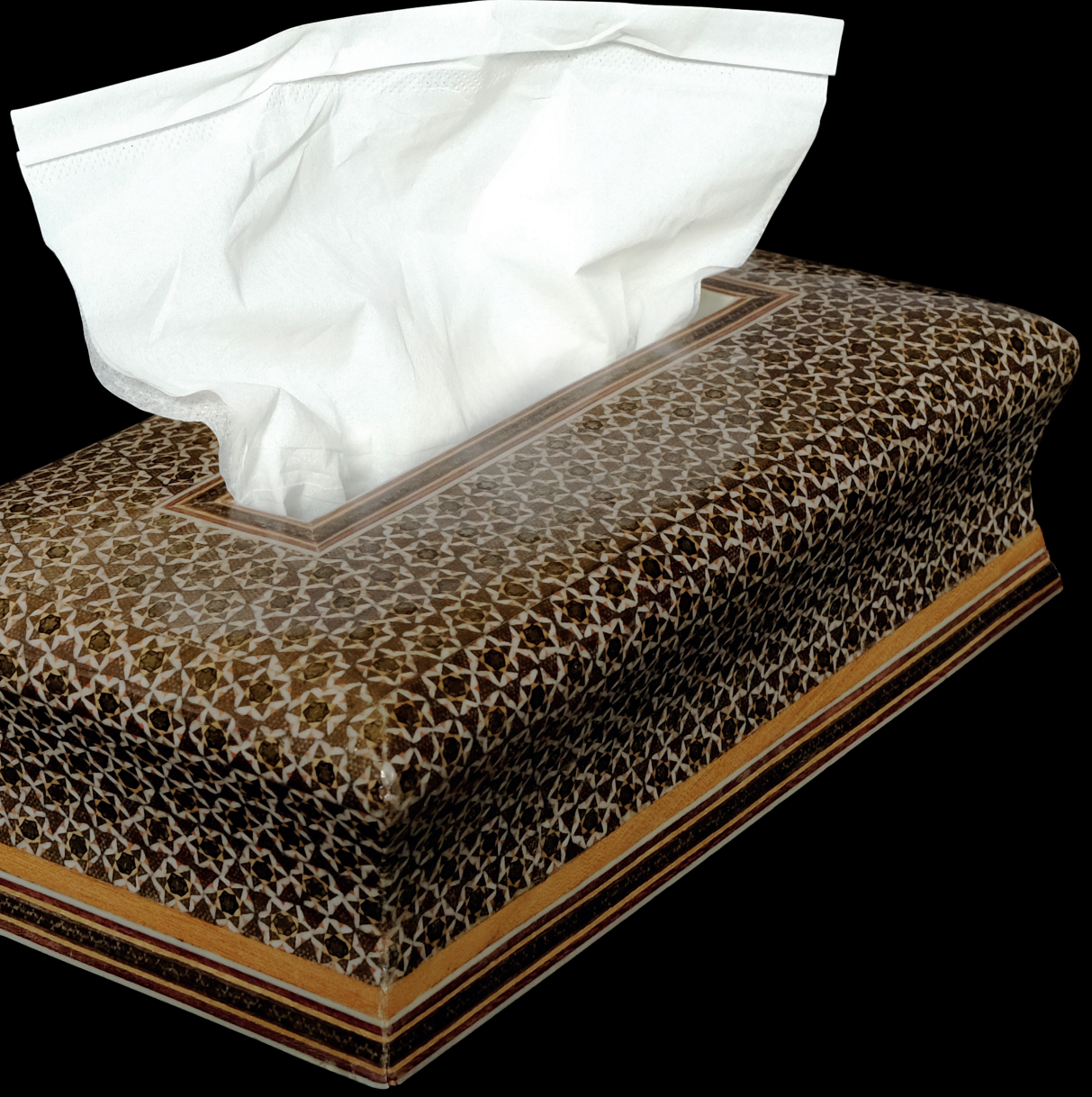




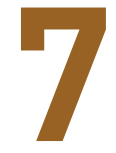

\section{PSYCHOSIS AS A CONFOUNDER OF SYMPTOM CREDIBILITY TESTING IN A TRANSCULTURAL SAMPLE}

Published as:

Van der Heide, D., Boskovic, I., Van Harten, P, \& Merckelbach, H. (2020). Psychosis as a confounder of symptom credibility testing in a transcultural sample. Journal of Forensic Psychiatry \& Psychology, 31, 64-75. https://doi.org/10.1080/14789949.2019.1663899 


\section{Abstract}

To check the credibility of impairments reported by refugees, so-called performance validity tests may be administered. We explored whether a psychotic condition may compromise performance on such test in patients admitted to a referral center for refugee mental health in the Netherlands $(n=231)$. We selected patients with no clear incentive to exaggerate their complaints $(n=80)$; psychotic patients in this subsample $(n=44)$ made significantly more errors on a simple forced-choice emotion recognition task than non-psychotic patients $(n=$ 36), means being $9.0(S D=6.8)$ and $5.9(S D=5.1)$, respectively. Next, we selected patients with an incentive to deny complaints $(n=24) ; 79 \%$ of these patients had a psychotic disorder. Their failure rate on the emotion recognition test (21\%) was the same as the failure rate of a control group of Dutch chronic psychotic patients $(n=47)$, but significantly above that $(2 \%)$ of a control group of healthy controls $(n=51)$. We regard this as preliminary evidence that psychotic symptoms and/or antipsychotic medication may put a constraint on testing symptom credibility in refugees. 


\section{Introduction}

Refugees seeking asylum may be tempted to distort psychiatric symptoms and impairments. That is, they may exaggerate or even fabricate them; attribute symptoms to nonexistent causes (e.g., by falsely claiming a traumatic history) or feign persistence of symptoms after successful treatment (Lipman, 1962). Faced with the dire consequences of expulsion, they may do so to comply with the criteria for medical asylum (Lustig, 2008). Using unstructured interviews, clinicians are poor in detecting distorted symptom presentations (DandachiFitzGerald et al., 2017). Hence, to screen for exaggerated, fabricated, falsely attributed or falsely maintained symptom presentations, dedicated tests known as Symptom Validity Tests (SVTs) have been recommended (Bush et al., 2005). Patients who engage in distorted illness presentations often fail on such tests (i.e., endorse rare or unlikely symptoms and/or perform below standards on a simple cognitive task), yet the reverse is not true: test failure does not necessarily indicate distorted illness presentation (Young, 2014; Merckelbach et al., 2019). Neither does a distorted symptom presentation rule out the presence of a genuine mental illness (Tracy, 2018).

There are a number of confounders (e.g., severe cognitive impairment; see e.g., Davis, 2018) that may suppress performance. In patients from ethnic minorities, cultural differences and language obstacles may cause failure on tests (see for a review: NijdamJones \& Rosenfeld, 2017). Some SVTs have been translated into other languages and appear to function adequately in ethnic groups for which they were not originally designed (e.g., Montes \& Guyton, 2014). However, for culturally diverse migrants such as refugees, translated and validated instruments are not always available. One way to deal with this problem is to administer tests that do minimally depend on language (Erdodi et al., 2017). Performance Validity Tests (PVTs), focusing on detection of exaggerated impairment, are a subtype of SVT with often a low verbal mediation. An example of such a PVT is Morel's Emotional Numbing Test (MENT; Morel, 1998), which was specifically designed to detect exaggeration of traumarelated symptoms, but has recently also become popular as a screener for a broad spectrum of symptom exaggeration (e.g., in soldiers who seek medical certification of occupational disability; Zimmerman et al., 2013). This test requires patients to discriminate between facial expressions of basic emotions (e.g., angry vs surprised). Prior to testing, patients are informed that persons with trauma-related problems often experience emotional numbing and that this may lead to mistakes in identifying other people's emotions. Typically, those who exaggerate 
their symptomatology produce considerably more errors on the MENT than genuine patients (see for a review, Ray, 2014). Some authors opine that tests such as the MENT hardly produce false positives because "they can be successfully solved by virtually any adult, with the exception of patients suffering from severe neurocognitive disorders related to word processing, visual acuity, spatial neglect, or face processing" (Merten et al., 2009, p. 286).

Van der Heide et al. $(2016,2017)$ used a forced-choice PVT modeled after the MENT to screen patients of a psychiatric facility for refugees in the Netherlands. Patients with an incentive to exaggerate failed the forced-choice test significantly more often than patients with no such incentive. Yet, a considerable minority of patients (25\%) with an incentive to deny impairment also attained error scores beyond the cut-off. Why do patients who have no reason to exaggerate their illness fail on a forced-choice PVT? In the studies of Van der Heide et al. $(2016 ; 2017)$, compulsory admission was regarded as an incentive to deny impairment, the reason being that symptom exaggeration would extend the compulsory admission. Could it be that psychotic patients were overrepresented in the negative incentive group, resulting in artificially inflated error scores on the forced-choice PVT? Evidence for this possibility can be found in the work of Dandachi-FitzGerald et al. (2011), who reported a similar rate of PVT failure (i.e., 25\%) in patients with psychotic symptomatology attending a non-forensic outpatient facility. On the other hand, Schroeder and Marshall (2011) administered several PVTs to patients with psychotic disorders $(n=104)$ and reported that they seldom failed on these tests, suggesting that PVTs are safe in this particular group. Likewise, Morel (1998) reported that schizophrenic patients made only few errors on the MENT, but his sample of schizophrenic patients was small $(n=17)$.

So, are psychotic symptoms an additional confounder when refugees are screened for distorted illness presentations, or not? To control for other major confounders, we selected patients without incentives to exaggerate from a referral center for refugee mental health and administered a PVT with low verbal mediation. For a preliminary answer to our question, we compared the error scores of psychotic and non-psychotic patients. Also, we checked whether a subgroup of patients with an incentive to deny impairments indeed contained a disproportionally large proportion of psychotic patients and compared their error scores and failure rates to those of a group of Dutch, chronic psychotic patients and a group of healthy controls. 


\section{Methods}

\section{Participants}

Our study sample was selected from patients who had been admitted to a national referral center for non-forensic refugee mental health in the Netherlands. The average duration of admission in this referral center varied between 6 and 9 months. All patients hospitalized in the study period (2008-2012) were invited to participate; only a few patients $(<5 \%)$ refused or were mentally unfit to give consent. Thus, 231 patients (172 men; 74\%) were included, of whom 104 patients (45\%) originated from Africa, 58 (25\%) from the Middle East, 46 (20\%) from the former USSR, 14 (6\%) from the Far East, and nine (4\%) from former Yugoslavia. Mean age was 34 years $(S D=11.7$; range: $15-67$ years). Of these patients, $63(27 \%)$ already obtained a residence permit; the others were still involved in a legal procedure for asylum.

A panel of social workers (see below) assigned 151 (65\%) inpatients to the group with positive incentives (incentives to exaggerate), 56 (25\%) inpatients to the group without incentives or with mixed incentives, and 24 (10\%) inpatients to the group with a negative incentive (an incentive to deny). All patients did the forced-choice emotion recognition test of this study, but only the error scores of the latter two groups were selected to test our hypothesis.

There are several centers for refugee mental health in the Netherlands. These facilities are specialized in the treatment of asylum seekers and refugees with resistant symptoms and/ or admit patients because the referring clinicians lack the expertise to treat this group. Most centers offer primarily trauma therapy. The referral center described in the current study was selected for our study because, unlike the other centers, it was part of the inpatient facilities of a general psychiatric hospital and as such provided the option of compulsory admission. Accordingly, although 95\% of the referred patients reported a history of traumatic events, they exhibited a much wider range of psychopathology than trauma-related symptoms, including psychotic symptomatology. Based on the diagnostic classifications (Diagnostic and Statistical Manual of Mental Disorders, 4th ed., text rev.; American Psychiatric Association, 2000) mentioned in their discharge letters, 67 patients (29\%) had a psychotic disorder (schizophrenia spectrum and other psychotic disorder), 66 (29\%) an anxiety disorder of whom 60 (26\%) PTSD, $38(16 \%)$ an affective disorder, and eight (3\%) a dissociative disorder. In 76 patients (33\%), symptoms were classified as adjustment disorder. In 51 cases (22\%), there was a co-morbid personality disorder, in 32 cases (14\%) a co-morbid substance use disorder, and in six cases (3\%) data were missing. 
As expected, there was an unequal distribution of psychotic symptomatology in the different incentive groups. Thus, 23 patients (15\%) in the positive incentive group, 25 (45\%) in the no/mixed incentive group, and 19 (79\%) in the negative incentive group had a psychotic disorder: Fisher-Freeman-Halton Exact $p<0.001$. Similarly, in the subgroup with positive incentives, six patients (4\%) were admitted by court order. In the no/mixed incentive and in the negative incentive group, these numbers were 20 (36\%), and 19 (79\%), respectively: Fisher-Freeman-Halton Exact $p<0.001$. Patients with psychotic symptoms were treated with either classic or atypical antipsychotics, except for those in the positive incentive group; their medication was as a rule tapered off.

The first control group consisted of native Dutch, chronic psychotic patients who were recruited from a long-stay psychiatric ward in another psychiatric hospital. In the Netherlands, this type of ward is reserved for patients with at least three continuous years of prior admission in other psychiatric wards. In total, 47 patients (33 men; 70\%) were included. Their mean age was 51 years ( $S D=11.7$; range: 25 - 69 years). In total, 42 patients (89\%) had a primary DSMIV-TR classification of schizophrenia; five patients (11\%) were diagnosed with a schizoaffective disorder. Their initial admission usually was by court order. All patients were treated with either classic or atypical antipsychotic medication.

The second control group consisted of healthy controls. They were professional translators who served as interpreters in the clinic where the subsample of asylum seekers was recruited. They were not screened for mental or cognitive disorders, under the assumption that major cognitive impairment and psychotic disorder are not prevalent among professional interpreters. The controls were invited to take the forced-choice test (see below) themselves before assisting with the administration of the test. A total number of 51 interpreters (28 men; 55\%) participated. Nineteen (37\%) interpreters originated from the Middle East, 17 (33\%) from Africa, five (10\%) from the former USSR, three (6\%) from the Far East, five (10\%) were Dutch or from Western Europe, and in two cases (4\%) information about the country of origin was missing.

\section{Instrument}

As a PVT with low verbal mediation, we used a research version of a forced-choice effort task modeled after the Morel Emotional Numbing Test (MENT; Morel, 1998); it was introduced to the patients in the same way as the MENT (see above), by one psychiatrist who was a staff member of both referral center and the long-stay ward for Dutch chronic psychotic patients. 
Our version (Geraerts et al., 2009) consisted of 20 colored slides of 10 facial expressions posed by a man and a woman. Their expressions reflected happiness, frustration, sadness, anger, fear, calmness, surprise, shyness, confusion, and sleepiness. The slides were presented on a computer screen $(30 \times 38 \mathrm{~cm}$ ). Being a forced-choice test, each trial consisted of a correct and an incorrect answer option. In the first series of 20 trials, patients had to indicate which of two words (e.g., "happy" versus "surprised") that were presented best described the facial expression in the picture. In the second run of 20 trials, patients were presented with two different expressions simultaneously and only one emotion word. They had to identify the expression that best matched the word. In the final run of 20 trials, patients were shown two photographs and two words in each slide and these had to be connected in the right way. Slides of words and basic expressions were presented with no time limitations and participants could inspect them as long as needed to come up with an answer.

Emotional labels used in the test were translated and back-translated into several languages. However, as some patients reported to be unable to read or write and some translations were not available in the native language of the patient (e.g., a Russian translation for all patients from former Soviet Republics), a professional interpreter was present during the test to assist with the instructions and the key verbal labels when necessary.

Test performance was defined in terms of the total number of errors summed across the three runs. Morel (1998) recommended a cutting score of nine errors on the MENT, with scores above this level raising the suspicion that impairment is exaggerated. In a sample of Croatian war veterans elevated error levels (i.e., > 9 errors) on a translated version of our forced-choice PVT were found to discriminate between treatment-seeking and compensationseeking veterans (sensitivity: 92\%; specificity: 96\%; Geraerts et al., 2009).

\section{Procedure}

As a matter of routine, a professional interpreter informed patients at the start of their admission to the referral center about the diagnostic procedures in the center: clinical interviews, observation, and psychological tests. They were also told that standard Western psychological tests might not yield useful results in patients with a different cultural background and that there would be an assessment in each individual case whether test results could be used or not. In addition, the patients were asked for consent for anonymous use of their data for scientific purposes. In case of unaccompanied minors, their legal representative was consulted. The study was approved by the Central Committee on Research Involving Human 
Subjects (CCMO).

Social workers of the hospital independently checked the medical files of the patients using a pre-defined list of incentives that may promote symptom exaggeration or denial of symptoms. One point was added for each condition potentially promoting exaggeration, such as: 1. An asylum procedure still in progress; 2. (Application for) a temporary refugee status issued for medical reasons; 3. Any other current procedure requiring a medical report indicating medical necessity, urgency or exemption (e.g., request for family reunion while the patient is not able to generate the necessary income demanded by Dutch law; a request for urgent change of housing or special housing arrangements; a request to be exempted from the criterion to pass a language test in the naturalization procedure). For each condition discouraging exaggeration the social workers subtracted a point. Such conditions would be: 1 . A compulsory nature of the present admission; 2. Any current procedure requiring a medical report indicating improved functioning or decreased need for medical treatment or scrutiny (e.g., a child custody procedure, a request for voluntary repatriation). Patients with one point or more were classified as having a positive incentive, patients with zero points were classified as having no/mixed incentives, and patients with minus one point or less were considered to have a negative incentive. Social workers evaluating incentive levels had no knowledge about the forced-choice PVT outcomes, and the psychiatrist who presented the forced-choice PVT was not aware of the incentive status assigned by the social workers. However, as a staff member, the psychiatrist was involved in the treatment of the asylum seekers and had access to their medical files; so, in this respect blinding was incomplete.

In the subsample of Dutch psychotic patients, the forced-choice task was administered as part of a project to introduce a set of standard cognitive tests in order to evaluate rehabilitation interventions. The patients were informed that one of the tests was administered to assess whether cognitive tests could provide useful information for rehabilitation purposes.

Healthy controls, the interpreters, were asked to complete the task to serve as an additional check on the translations of the emotion words in the forced-choice task.

\section{Data analysis}

Because our data were not normally distributed (Shapiro-Wilk W's >0.879, $p^{\prime} s<0.01$ ), we used descriptive and non-parametric statistics (Kruskal-Wallis and Mann-Whitney U tests) to evaluate errors on the forced-choice PVT in the subsamples. We used Fisher exact $p$ to compare failure rates in subsamples, with failure defined as error rates $>9$ errors. 


\section{Results}

The mean number of errors on our PVT in referral center patients (subsample 1) was $18.2,95 \% \mathrm{Cl}=17.0-20.7$, with a range of 0 to 54 errors; 142 patients (61\%) made more than nine errors. Looking at the different incentive groups, patients with a positive incentive made on average 24.8 errors ( $41 \%$ of the items), $95 \% \mathrm{Cl}=22.5-27.1$, with a range of 0 to 54 errors; 121 (80\%) of these patients made more than nine errors. Patients with no incentives or mixed incentives made on average 8.2 errors ( $14 \%$ of the items), $95 \% \mathrm{Cl}=6.3-10.1$, with a range of 0 to 29 errors; 16 (29\%) of them made more than nine errors. Patients with a negative incentive made on average 6.3 errors ( $11 \%$ of the items), $95 \% \mathrm{Cl}=5.0-7.6$, with a range of 0 to 12 errors; five $(21 \%)$ made more than nine errors. A Kruskal-Wallis test indicated that the error scores of the three incentive groups were significantly different, $\chi^{2}(2)=78.2, p<0.01, \eta^{2}=0.34$.

First, we compared psychotic patients of the referral center in the combined no/mixed and negative incentive groups $(n=44)$ with non-psychotic patients $(n=36)$ in these groups. Psychotic patients had higher error scores on the forced choice PVT than the non-psychotic patients, means being $9.0(S D=6.8)$ and $5.9(S D=5.1)$. A Mann-Whitney $U$ test indicated that this difference was significant: $U=525.5, z=2.59, p<0.05, \eta^{2}=0.08$. Using the cutoff of nine errors, 14 (32\%) of the psychotic patients failed the test, whereas seven (19\%) of the non-psychotic patients did, a difference that did not attain significance, probably due to the small sample sizes involved (Fisher exact $p=0.21$ ).

Next, we compared the patients with a negative incentive to the two control groups. In the control group of Dutch psychotic patients the mean number of errors was $5.7,95 \%$ $\mathrm{Cl}=4.4-7,0$, with a range of 0 to 24 errors; ten (21\%) patients in this subsample made more than nine errors. To investigate whether the error scores of chronic psychotic patients differed from those of patients of the referral center with a negative incentive, we conducted a MannWhitney $U$ test: the error levels in both groups were similar, $U=483.0, z=0.99, p=0.32$.

In the healthy controls group, the mean number of errors was $3.4,95 \% \mathrm{Cl}=2.7-4.1$, with a range of 0 to 12 errors; one person (2\%) made more than nine errors. The error scores of healthy controls were significantly lower than those of referral center patients with a negative incentive and the Dutch psychotic patients: $U=300.5, z=3.56, p<0.01, \eta^{2}=0.41$ and $U=$ 821.0, $z=2.70, p<0.01, \eta^{2}=0.07$ respectively (corrected alpha level $\left.=0.017\right) .{ }^{1}$

1) Note: Error scores exceeding chance level (> 36) only emerged in the subsample of referral center patients with a positive incentive. In this subsample, 38 patients (16\% of all referral center patients) had such a score. 


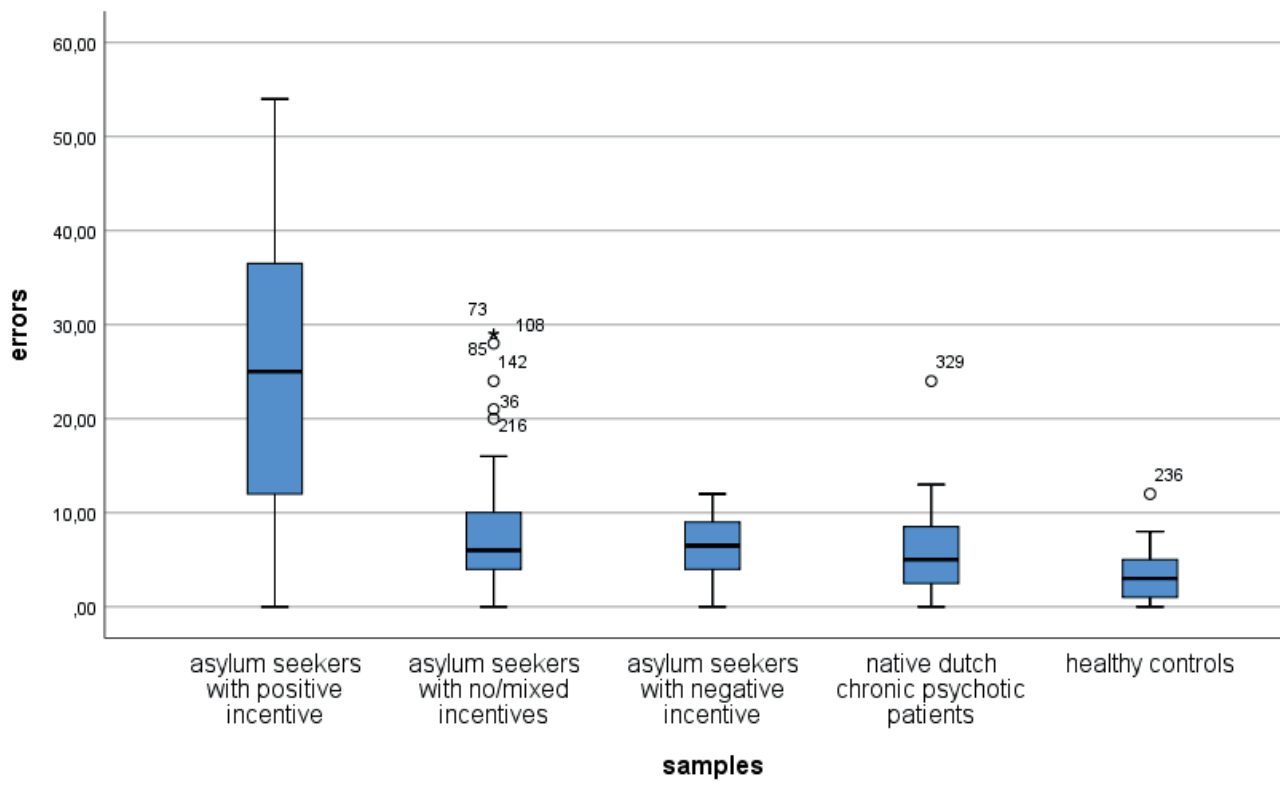

Figure 1. Numbers of errors on the forced-choice task in subsamples. Cut-off > nine errors.

\section{Discussion}

Our findings suggest that psychotic symptoms may act as a confounder when refugees are screened for distorted illness presentations. First, in the combined no/mixed and negative incentive subsamples of the referral center, psychotic patients had higher error scores on the forced-choice PVT than non-psychotic patients. This finding is congruent with Hunt et al. (2014), who found elevated error scores on PVTs in patients with schizophrenia and schizoaffective disorder (see also Glassmire et al., 2016; Peters et al., 2013), and also with studies that demonstrated a relative impairment of patients with schizophrenia in emotional recognition in facial expressions (for an overview, see Edwards et al., 2002).

Our first finding is substantiated by our second: referral center patients with an incentive to deny their impairment, most of whom (79\%) were classified as having a psychotic disorder, made significantly more errors than healthy controls on a PVT, whereas they performed at a similar level as native Dutch, chronic psychotic patients. As a matter of fact, a non-trivial minority-21\%- of both groups failed on the forced-choice test. As neither of these two patient groups had obvious motives to exaggerate, it is unlikely that their errors reflect lack of effort 
and/or an attempt to misrepresent their impairments. Psychotic symptomatology was a prominent feature in both groups, which was not the case in the other groups (the other incentive samples of the referral center and healthy controls). Thus, one distinct possibility is that psychotic symptomatology may artificially raise failure rates on forced-choice PVTs of the type used in in the current study.

Third, replicating earlier studies (Van der Heide et al., 2016, 2017), referral center patients with a positive incentive made significantly more errors on the forced-choice PVT than patients with a mixed or a negative incentive to produce deviant scores. This indicates that our PVT was sensitive to incentives. Yet, combined with the other findings, the conclusion must be that raised error scores do not necessarily point in the direction of intentional symptom distortion motivated by positive incentives because psychotic patients without incentives may also exhibit raised error scores.

It may well be the case that impairments that are central to psychosis, notably disturbances in semantic memory, visual memory, verbal learning, and attention (e.g., Saykin et al., 1991), interfere with the cognitive efficiency required to conduct even a relatively easy task such as a forced-choice PVT. Also, negative symptoms such as apathy and avolition may contribute to suboptimal performance on a PVT (Gorissen et al., 2005). Another possibility is that medication, at least conventional antipsychotic drugs, may exacerbate cognitive impairments to such degree that these impairments suppress performance on simple cognitive tasks (e.g., Kasper \& Resinger, 2003). The extent to which both factors, disturbances inherent to psychosis and side-effects of medication, are prominently present in samples of psychiatric patients may explain why some researchers reported relatively low rates of PVT failures in psychotic patients (Schroeder \& Marshall, 2011), whereas others found considerable proportions failing PVTs (e.g., Van der Heide et al., 2016, 2017).

A strength of our study is that almost all referral center patients consented to participate, which makes it unlikely that hidden selection effects distorted our results. However, a number of limitations of the present study are important to discuss. First, a limitation is that social workers only checked the files for the presence or absence of documented incentives (e.g., being involved in a custody dispute). We did not look into the presence or absence of other types of incentives, the types that are more difficult to objectify and that are usually not mentioned in medical files, such as previous experiences of care being denied. Second, the referral center patients were the only group for whom external incentives were taken into consideration. We assumed that the other groups would have no such incentives, but we cannot rule out 
their presence. For example, one Dutch patient with a particularly high error score informed the nurses that he was anxious that a low error score might result in relocation to another facility with less care. Third, the fact that mean error scores and failure rates of the negative incentive group and those of Dutch chronic psychotic patients were highly similar does not imply that the error scores of referral center patients were unaffected by cultural factors (e.g., different test taking attitudes; Ardilla, 2005). Clearly, the presence of one confounder does not necessarily rule out the presence of another. Finally, in our study we relied on diagnostic classification and did not collect specific symptom severity data. Determining at which levels of symptom severity performance on symptom credibility tests becomes compromised has considerable practical value for clinicians who want to know whether it is safe to administer PVTs or any other tests to evaluate the credibility of symptoms for that matter. Future studies could systematically relate PVT scores to measures of psychotic symptom severity, such as the Positive and Negative Symptoms Scale (PANSS; Kay et al., 1987), to cognitive aberrations typical for psychosis (e.g., lack of attentional control), and to antipsychotic drug use, preferably in patients motivated to perform optimally.

\section{Conclusions}

In sum then, refugees with no obvious incentive to exaggerate their impairments may still fail a simple forced-choice PVT. Our study offers preliminary evidence that, apart from cultural and linguistic differences, current psychotic symptoms may be a confounder when PVT tests are administered to this group. The implication of this is straightforward: clinicians should be cautious in administering PVTs to refugees with psychotic symptoms and the presence of such symptoms may be a reason to set cutoffs at more stringent levels and to use multiple PVTs (Schroeder \& Marshall, 2011). 



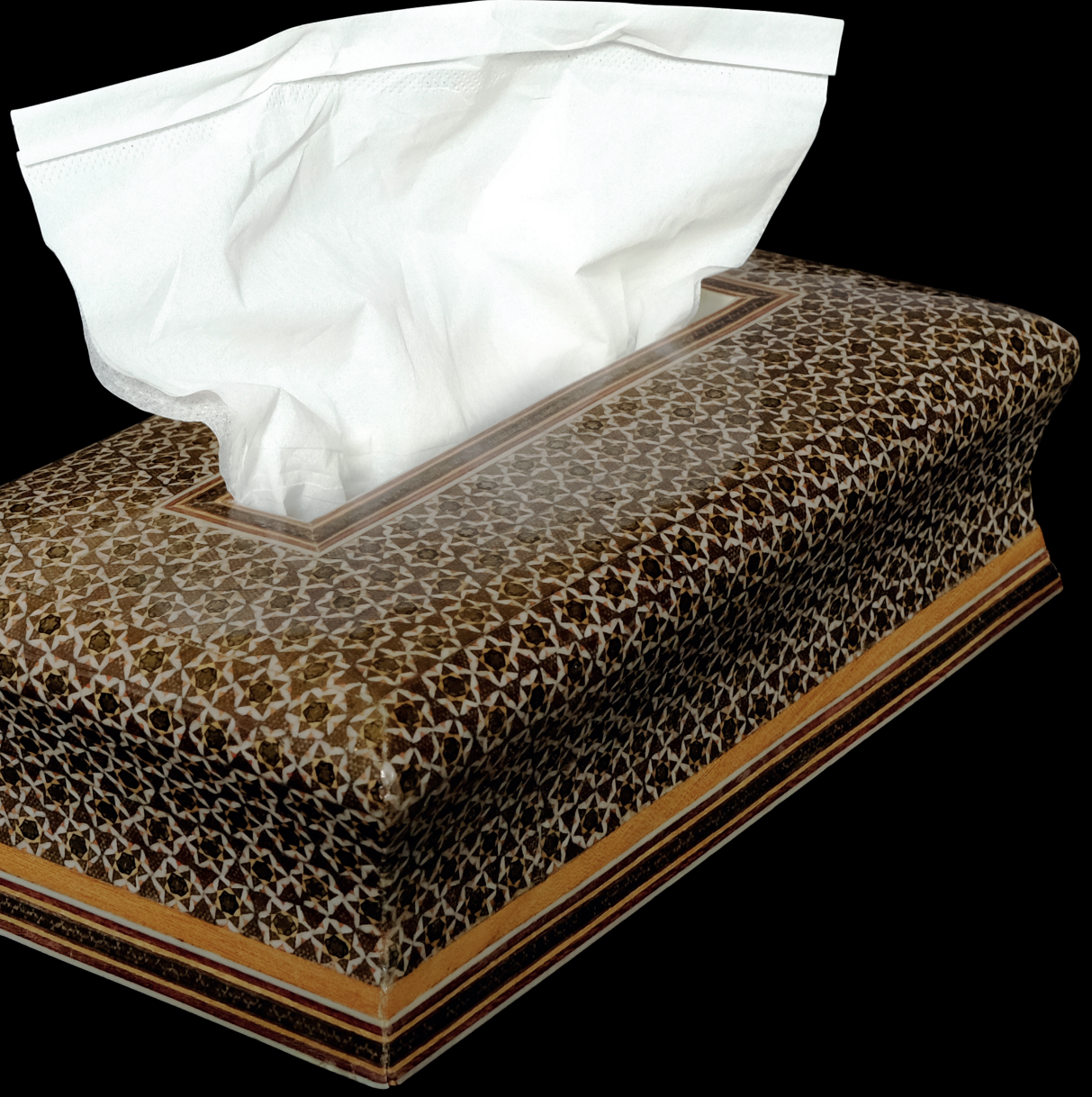




\section{8}

\section{THE FLOOR EFFECT STRATEGY FOR THE ASSESSMENT OF PERFORMANCE VALIDITY IN REFUGEE MENTAL HEALTH PATIENTS}

Submitted as:

Van der Heide, D., Boskovic, I., \& Van Harten, P. (2020). The floor effect strategy for the assessment of performance validity in refugee mental health patients. 


\section{Abstract}

Performance Validity Tests (PVTs) are designed to distinguish between exaggerated and genuine cognitive impairment. PVTs include tasks that induce a mild cognitive load, and genuinely impaired patients can perform this task with sufficient effort, resulting in the 'floor effect'. However, it is unknown whether this effect also occurs when PVTs are used to assess culturally diverse, mixed-psychiatric populations, such as those found in referral centers for refugee mental health. We therefore studied test outcomes of patients $(n=56)$ in a referral center for refugee mental health in the Netherlands. We compared patients' scores on a standard cognitive test with their performance on two PVTs; a forced-choice task modelled after the Morels Emotional Numbing Test (MENT), and Tombaugh's Test of Memory Malingering (TOMM). Patients were divided into three groups: those with a positive incentive to exaggerate impairment, those with no/mixed incentives to exaggerate, and with a negative incentive (i.e., an incentive to minimize or deny impairment). We found that both PVTs distinguished between incentive groups, but the regular cognitive test did not. The floor effect thus still occurs when PVTs are used to assess culturally diverse, mixed-psychiatric populations, such as those found in referral centers for refugee mental health. 


\section{Introduction}

Mental health issues are highly prevalent among persons seeking asylum in Western Europe. Laban et al. (2005) found a prevalence of $42.0 \%$ of one or more mental disorders among persons from Iraq seeking asylum in the first six months after they arrived in the Netherlands.

Information about a person's mental health plays an important role in the asylum applications in the Netherlands. First, to prevent undue rejection of mentally disorganized applicants, persons seeking asylum may be examined for mental health issues prior to interviews with immigration services. Second, asylum legislation allows the use of a medical examination as supportive evidence. Third, if their regular asylum procedure is unsuccessful, persons seeking asylum can apply for a residence permit on humanitarian grounds. Such grounds would include a serious illness that cannot be treated in the country of origin or that impairs their ability to travel. These regulations cover claims of mental disorders, as well as of the risk of suicide if asylum is not granted (ECRE, 2020; Meffert et al., 2010).

Due to these provisions, some applicants may be tempted to exaggerate, or even feign, some or all of their symptoms (Lustig, 2008). The act of intentional exaggeration or full fabrication of symptoms in order to obtain an external benefit is defined as malingering (DSM-5; American Psychiatric Association, 2013). To screen for such response bias, Symptom Validity Tests (SVTs) can be used (Bush et al., 2015). In migrants, differences in culture and language may confound the outcomes of SVTs (see the review by Nijdam-Jones \& Rosenfeld, 2017). Some SVTs have been translated and validated for ethnic groups for which they were not originally designed (e.g., DuAlba \& Scott, 1993; Montes \& Guyton, 2014; Vilar-Lopez et al., 2007). However, in culturally diverse settings, such as referral centers for refugee mental health in Western European countries, translated and validated instruments are often unavailable. Staff of such centers may try to cope with this situation by employing dialogue interpreters, but this method has been shown to be error-prone (Bot, 2005; Correa, 2018). Another option is to use tests with low verbal mediation (Benuto et al., 2014; Erdodi et al., 2017).

One subcategory of SVTs with low verbal mediation is the Performance Validity Test (PVT). This type of SVT focusses specifically on the exaggeration of impairment (DandachiFitzGerald et al., 2011; Martin et al., 2015). Previous research has shown that patients who fail on PVTs (i.e., perform below standards) often engage in malingering (Merten \& Merckelbach, 2013; Young, 2014). Evaluators use the 'PVT floor effect' to distinguish between genuine and exaggerated impairment. In statistics, a floor effect arises when a data-gathering instrument 
has a lower limit to the data values it can reliably specify (Lim et al., 2015). This lower limit is referred to as a floor. The tasks included in the PVTs induce a cognitive load that allows patients with genuine impairments to perform relatively well with enough effort. Error scores above a predefined cut-off are therefore regarded as not credible. This implies, that the cognitive load needs to be tailored to the target group at hand. The cognitive load induced by a standard PVT may be too high for some more vulnerable patient groups. For example, psychotic symptoms (Schroeder \& Marshall, 2011), intellectual disability (Shandera et al., 2010), alexithymia (Merckelbach et al., 2018) are known confounders of PVTs. Mcwhirter et al. (2020) reviewed PVT studies in clinical populations with psychiatric disorders, intellectual disability, degenerative brain disease, brain injury, functional disorders and epilepsy, and found failure rates for some groups and tests exceeding $25 \%$. This finding implies that PVTs might be sensitive to genuine co-morbidities that could confound the PVT outcomes.

Patients who are referred to centers that specialize in refugee mental health in the Netherlands often suffer from severe, therapy-resistant psychiatric symptoms, have a limited proficiency in the language of the host country, and are culturally diverse. In these patients, PVT outcomes might be confounded by all of these issues, although, this has not yet been thoroughly tested. In the present study, we therefore determined whether the floor effect still occurs when PVTs are used in culturally diverse, mixed-psychiatric populations such as those found in referral centers for refugee mental health.

\section{Method}

\section{Participants}

Participants were patients admitted to a non-forensic psychiatric referral center for refugee mental health in the Netherlands and included patients with and without refugee status. Patients were referred because of severe, therapy-resistant psychopathology due to a variety of disorders. This usually involved Posttraumatic Stress Disorder, but also major affective disorder, psychotic disorder, personality disorder or substance-related disorder. Some patients also suffered from mild traumatic brain injury or from developmental disorders causing mild degrees of intellectual impairment. All patients admitted to the referral center in the study period were included if they were willing and able to give informed consent for participation in the study and for the use of their anonymized data for scientific purposes. Fewer than $5 \%$ 
of the inpatients were unwilling to give their informed consent or were unable to do so. The study was approved by the Central Committee on Research Involving Human Subjects (CCMO).

A total of 56 patients completed a simple forced-choice task modelled after Morel's Emotional Numbing Test (MENT; Morel, 1998), the Test of Memory Malingering (TOMM; Tombaugh, 1996), and a standard cognitive test, i.e. the Study Path Selection Test (SPST; Bureau InterCulturele Evaluatie, 1998). Mean age was 30.3 years $(S D=13.2), 17$ were female $(30 \%)$, 22 (39\%) originated from Africa, 19 (34\%) from the Middle East, 12 (22\%) from the former USSR, and 3(5\%) from Eastern Asia. In our analysis of their scores, we differentiated between incentives to exaggerate impairment (i.e., through suboptimal effort); 39 (70\%) inpatients were classified as having a positive incentive, 11 (19\%) as having no/mixed incentives, and six (11\%) as having a negative incentive to exaggerate impairment (i.e., an incentive to minimize impairment).

\section{Measures}

Forced-choice task. This test was devised to signal the exaggeration of impairment following psychological trauma. It is a forced-choice task involving the identification of basic emotional expressions, modelled after the Morel Emotional Numbing Test (MENT; Morel, 1998), which is sensitive to response distortion in the assessment of trauma-related problems. Our version consisted of 20 colored pictures of 10 facial expressions posed by a man and a woman. Their expressions reflected happiness, frustration, sadness, anger, fear, calmness, surprise, shyness, confusion, and sleepiness (Geraerts et al., 2007). The pictures were presented on a computer screen $(30 \times 38 \mathrm{~cm})$. Before the test, patients were told that emotional numbness is a prominent symptom of trauma-related problems and that this may cause people to have difficulties with the recognition of facial expressions. The rationale behind this instruction is that individuals who want to overstate trauma-related symptoms may intentionally produce more errors on this simple test. After this, patients were instructed to identify the emotion that best matched the expression of the face in three runs of 20 trials; one with one picture and two labels, one with two pictures and one label, and the last one with two pictures and two labels. Their task was to connect these stimuli in the right way. The emotion labels used in the test were translated and back-translated into several languages. Errors were summed across the three runs. There were no missing data. Cronbach's alpha was 0.94. Morel recommended a cutting score of nine errors on the MENT, with scores above this level indicating possible underperformance. In a sample of Croatian war veterans, elevated 
error levels (i.e., > 9 errors) on a translated version of our forced-choice task were found to discriminate between treatment-seeking and compensation-seeking veterans (sensitivity: 92\%; specificity 96\%; Geraerts et al., 2009).

Test of Memory Malingering. The Test of Memory Malingering (TOMM; Tombaugh, 1996) was devised to detect the exaggeration of memory impairment. Patients were asked to memorize 50 pictures that were presented to them on paper. The pictures were presented one by one during a time frame of three seconds for each picture. The first two runs were followed by a forced-choice task; they were again shown the same 50 pictures, but this time each picture was accompanied by a new picture on the same page: and each time, the patient had to identify the original picture. After the second run, they were given a break of 15 minutes, which was again followed by the forced-choice task, with the original pictures accompanied by new pictures on the same page. A patient with genuine impairments will pass this test due to the robust nature of visual recognition memory. In the TOMM protocol, the correct answers are summed for each consecutive run. Tombaugh recommends a cutting score of 45 correct answers for the third run. There were no missing data. Cronbach's alpha was 0.99.

Study Path Selection Test. The Study Path Selection Test (Trajectkeuze Toets; SPST) was developed in 1995 by the Dutch Agency for Intercultural Evaluation as part of the intake procedure for a Dutch language course for non-Dutch speaking immigrants (the 'NT2' course). It was revised in 1998 and pre-tested on more than 300 students. It was found to have a Cronbach's alpha ranging between 0.89 and 0.96 (Bureau InterCulturele Evaluatie, 1998).

The SPST was designed to assess cognitive skills needed to acquire proficiency in Dutch. It distinguishes between fast learners and slow learners with the aim of tailoring the course to the needs of the students. It has 25 items that require the student to sequence, rank, and recognize analogies between numbers, letters, and other symbols. To take the test, a minimal level of alphabetization in Latin script is required. The maximum time allowed to complete the test is 30 minutes. The score is defined as the total number of correct answers; a score of 10 or less indicates a slow study path, and a score of 15 or more a fast path. In the present study, the teachers allowed extra time, but many patients did not manage to complete the test. As a result, Cronbach's alpha could not be calculated. For the analysis of the data, only the correct answers were scored, disregarding not completed items and the extra time allowed.

Incentives to exaggerate impairment. Social workers employed at the hospital independently evaluated incentive levels of patients on the basis of file information. They differentiated between the presence of a positive incentive to exaggerate impairment, the 
absence of an incentive to do so, and the presence of a negative incentive (i.e., an incentive to hide or minimize impairment). They did this by adding one point for each condition potentially promoting exaggeration. Examples of such conditions include the following: an asylum procedure still in progress, temporary refugee status issued for medical reasons, or any other current procedure necessitating a medical report indicating medical necessity, urgency or exemption (e.g., request for family reunion while the patient is not able to generate the necessary income demanded by Dutch law; a request for urgent change of housing or special housing arrangements; a request to be exempted from the demand to pass the language test in the naturalization procedure, etc.). For each condition discouraging exaggeration they subtracted one point. Examples of such conditions include compulsory admission or any ongoing procedure necessitating a medical report indicating improved functioning or decreased need for medical treatment or scrutiny (e.g., a child custody procedure, a request for voluntary repatriation, etc.). Patients with a positive score of at least one point were considered to have a positive incentive, and patients with a negative score of at least one point were considered to have a negative incentive. Patients with zero points were considered to have no incentives, although some actually had mixed incentives. The social workers were blinded to the test outcomes.

\section{Procedure}

To avoid possible confounding by the order in which tests were presented, this was done in a counterbalanced way. The participants were assisted by both a staff member of the referral center and a professional interpreter to help them with test instructions. Staff members who assisted with test instructions for the PVTs were not aware of the information obtained by the social workers or the outcomes of the standard test. However, these staff members were also involved in the treatment of the patients and had access to their medical files. In that respect, blinding was less than complete.

\section{Data analysis}

We used descriptive statistics to evaluate the test outcomes. As our data were skewed or not evenly distributed (Shapiro-Wilk $W=0.892, p<0.001, W=0.851, p<0.001$, and $W=$ $0.946, p<0.05$, respectively) we used Kruskal-Wallis and Spearman's rho tests. 


\section{Results}

\section{Forced-choice task}

The mean number of errors was 16.5, 95\% Cl [13.1, 20.0] with a range of 0 to 41. In total, 34 patients (61\%) scored above the cut-off. A Kruskal-Wallis test showed that group differences based on incentives were significant: $\chi^{2}=17.22, d f=2, p<0.001$ (see Table 1). Of the patients with a negative incentive, or no/mixed incentives, $23 \%$ scored above the cut-off.

\section{Test of Memory Malingering}

The mean number of correct answers in the third run was $36.8,95 \% \mathrm{Cl}[33.2,40.5]$ with a range of 8 to 50 . The majority of patients $(n=31 ; 55 \%)$ scored below the cut-off. A Kruskal-Wallis test showed that group differences based on incentives were significant: $\chi^{2}=$ 27.88, $d f=2, p<0.001$ (see Table 1). Of the patients with a negative, or no/mixed incentives, $20 \%$ scored below the cut-off.

\section{Study Path Selection Test}

The mean number of correct answers was $9.3,95 \% \mathrm{Cl}[7.8,10.9]$ with a range of 1 to 24. 32 patients (57\%) qualified as slow learner (score 0-15), 13 patients (23\%) as fast learner (score 15-25), and 11 patients (20\%) had intermediate scores (11-14). A Kruskal-Wallis test showed that group differences based on incentives were not significant: $\chi^{2}=0.14, d f=2, p=$ 0.934 (see Table A). The error scores of the forced-choice task correlated with the scores on the TOMM at Spearman's $r h o=0.698, p<0.001$. All patients who failed the forced-choice task also failed the TOMM. The SPST did not correlate with the other tests. 
Table 1. The scores of refugee mental health patients on the forced-choice task, the TOMM ${ }^{\mathrm{a}}$ and the SPST ${ }^{b}$, The patients are divided in groups with either a negative incentive to exaggerate impairment (i.e., an incentive to minimize impairment), with no/mixed incentives to do so, or a positive incentive to exaggerate.

\begin{tabular}{lcccc}
\hline & $\begin{array}{c}\text { Negative incentive } \\
n=6\end{array}$ & $\begin{array}{c}\text { No/mixed incentives } \\
n=11\end{array}$ & $\begin{array}{c}\text { Positive incentive } \\
n=39\end{array}$ & $X^{2}$ \\
& Mean (SD) & Mean (SD) & Mean (SD) & \\
\hline $\begin{array}{l}\text { Forced-choice task } \\
\text { (Number of errors) }\end{array}$ & $7.7(5.5)$ & $5.7(3.2)$ & $21.0(12.8)$ & $17.222^{* *}$ \\
$\begin{array}{l}\text { TOMM } \\
\text { (Number of correct } \\
\text { answers, third run) }\end{array}$ & $50.0(0.0)$ & $49.3(1.5)$ & $31.3(12.7)$ & $27.878^{* *}$ \\
$\begin{array}{l}\text { SPST } \\
\text { (Number of correct } \\
\text { answers) }\end{array}$ & $8.8(7.4)$ & & & \\
& & & & \\
\hline
\end{tabular}

${ }^{* *} p<0.01$ (Kruskal-Wallis); ${ }^{\mathrm{a}}$ Test of Memory Malingering; ${ }^{\mathrm{b}}$ Study Path Selection Test

\section{Discussion}

In this study, we investigated whether the floor effect still occurs in persons seeking asylum who are referred to a refugee mental health center because of severe psychiatric pathology. The data we obtained confirm that the floor effect occurs in this target group, despite the confounding effects of cultural and linguistic diversity and psychiatric morbidity. Our findings can be summarized as follows:

First, both PVTs distinguished between incentive groups. Patients with a positive incentive to exaggerate impairment made more errors than patients without such incentives. The SPST did not distinguish between incentive groups: the groups earned similar scores on average.

Second, the outcomes of both PVTs correlated. This is precisely what one would expect if both tests measure impairment exaggeration rather than emotion recognition (forced-choice task) or memory (TOMM).

Third, more than half of the patients in our sample failed both SVTs. Only the patients in the no/mixed and negative incentive groups failed at a rate that is comparable with the rate reported by Mcwhirter et al. (2020) in clinical, mixed psychiatric groups. 
From the first finding, i.e. that the PVT outcomes were associated with external incentives and the outcomes of the standard cognitive test were not, it follows that the cognitive load presented by the PVTs was mild enough for patients with genuine impairment to pass with sufficient effort. The floor effect thus occurred as it was devised to do.

Regarding the correlation between PVT outcomes, other studies in mixed psychiatric samples patients were usually limited to a single dimension of illness distortion (DandachiFitzGerald et al., 2011; Dandachi-FitzGerald et al., 2016). Although a strong negative response bias could generalize into more than one type of impairment, the Dutch asylum procedure could also provide incentives to exaggerate impairment attributed to psychological trauma as well as memory impairment. Persecution, violence and human rights abuse in general are linked to psychological trauma but are also criteria to qualify for asylum, thus creating an incentive to fail items on the forced-choice task. Another part of the procedure for asylum focuses on the journey to the host country. Persons seeking asylum tend to be reluctant to divulge details about their journey; as human trafficking is a criminal offence, traffickers discourage this, and relatives of the person seeking asylum may need to use the same route in the future (Van Veldhuizen, 2017). This may be an incentive to exaggerate memory impairment, such as in the TOMM.

Regarding the large proportion of test failures, one could assume that a majority of patients were engaging in malingering. However, we believe that such a conclusion is premature. First, a malingering rate of more than half would be very high. For example, in their frequently cited study, Mittenberg et al. (2002) reported a base rate of malingering in externally motivated patients of $38.5 \%$. However, estimates tend to vary due to definitional and conceptual ambiguities. In a more recent, comprehensive review, Young (2015) reported a rate of $10 \%$ to $20 \%$ for forensic contexts. Second, the differential prevalence design that we used in our study is based on presumed incentives, and is devised only to establish a possible association; it cannot be used to estimate the extent to which patients in a sample engage in malingering (Rogers, 2018). Our findings thus indicate that the floor effect still occurred when differentiating between genuine and exaggerated impairment in the study sample, but they do not allow for any conclusions as to its accuracy in this respect. Third, test outcomes alone cannot be used for a classification of malingering. Ideally, findings would result from a multimodal approach that includes additional modes of investigation, such as structured interviews and observations, according the "Slick criteria" (Slick et al., 1999; Sherman et al., 2020).

Our study has also several limitations that should be noted. First, in our design we only 
counted objectifiable, external incentives. However, patients may also be internally motivated to exaggerate impairment as in factitious disorder, for example to receive attention and care in a hospital setting (Yates et al., 2018). Second, the extent to which persons seeking asylum in the Netherlands engage in symptom distortion is still a matter of speculation. The findings of this study concern a small sample of a highly selected patient group which is not representative for refugee mental health patients in general. Screening other groups of refugee mental health patients, such as persons seeking asylum in the initial stages of their procedure, might be a useful direction for future research.

\section{Conclusion}

In this sample of patients admitted to a referral center for refugee mental health because of severe, therapy-resistant symptoms, both PVTs still differentiated between incentive groups despite cultural differences and psychiatric morbidity. The standard cognitive test, with its more challenging cognitive load, did not differentiate. Our study shows that a floor effect still occurs when PVTs are used to assess patients at a referral center for refugee mental health. 


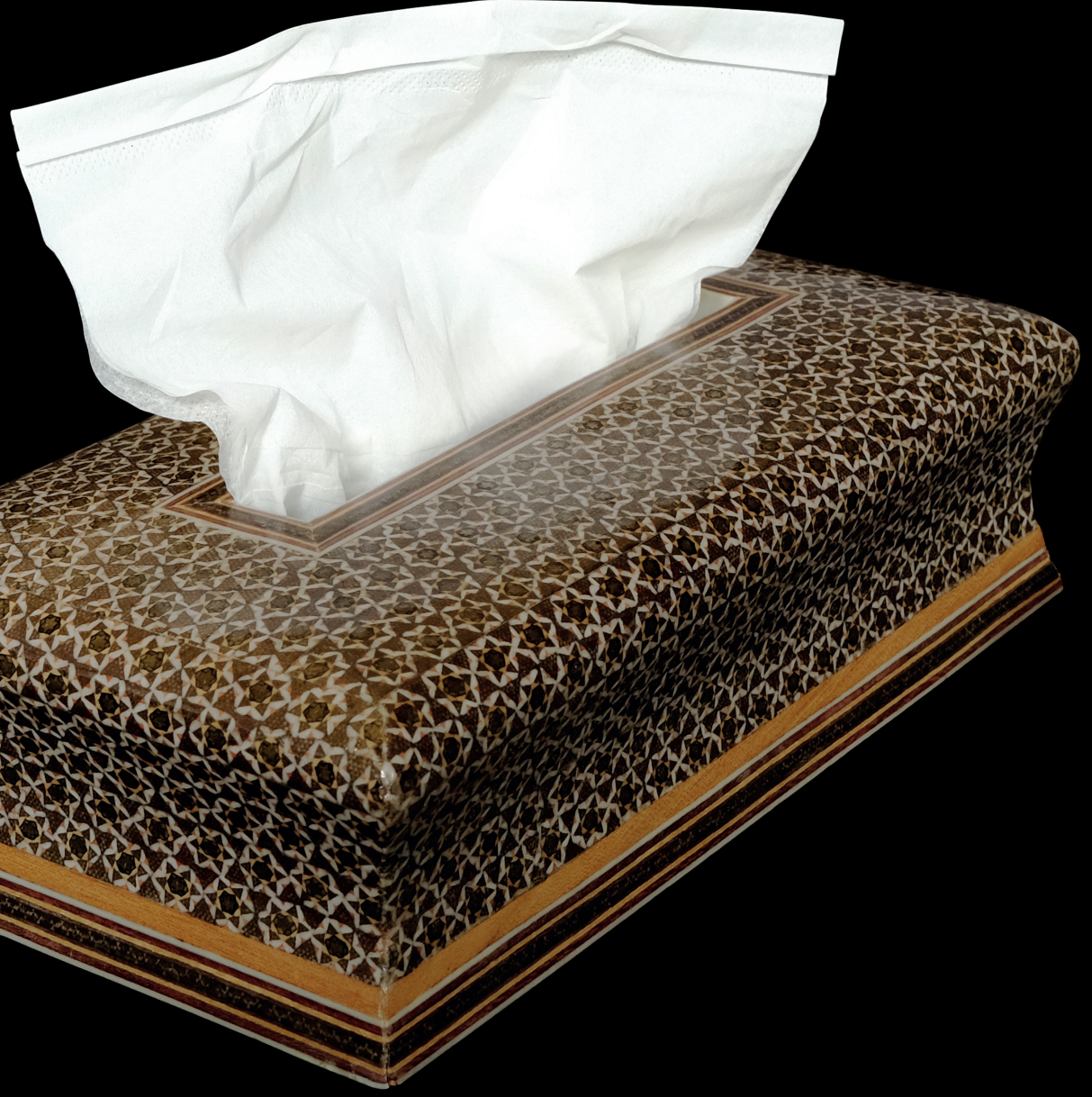




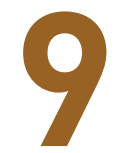

\section{DOES GEOGRAPHY CONFOUND PERFORMANCE VALIDITY TEST OUTCOMES?}

Van der Heide, D., Boskovic, I., \& Van Harten, P. (2020). Does geography confound performance validity test outcomes? 


\section{Abstract}

To assess symptom validity in refugee mental health patients, Performance Validity Tests (PVTs) may be preferred over Self-Report Validity Tests (SRVTs) because PVTs have lower verbal mediation. However, it has yet to be investigated whether patients' performance (i.e., effort) on PVTs varies depending on their geographical origin. If so, refugee mental health patients may be at risk for misclassification by PVTs as these tests were devised for American or European patients. We therefore compared SRVT and PVT outcomes of 203 patients from five geographical regions in the world at a refugee mental health center in the Netherlands. To ensure a valid comparison, we also looked for associations with poor proficiency in Dutch and external incentives to exaggerate impairment. Patients failed the SRVT more often than the PVT but, surprisingly, poor proficiency in Dutch was only associated with PVT outcomes. When only patients without external incentives were included, we found no significant differences between patients with poor and good Dutch proficiency, regardless of their geographical origin. We thus found no support for the hypothesis that geographical differences confound PVT outcomes in refugee mental health. 


\section{Introduction}

Mental disorders are highly prevalent among persons seeking asylum. For example, Laban et al. (2005) found a prevalence of $42 \%$ of one or more mental disorders among persons from Iraq seeking asylum in the first six months after they arrived in the Netherlands. Establishing the presence of mental health issues is important in applications for asylum in the Netherlands. The usual asylum procedure is as follows. First, applicants are offered a medical examination to assess whether they can be interviewed. This should prevent undue rejection of mentally disorganized applicants. Second, national legislation guarantees that medical examinations can be used as evidence to support a claim for asylum. Should the immigration services decide that this is not necessary, a person seeking asylum can still apply for a free-of-charge examination by the Institute for Human Rights and Medical Assessment (iMMO), an NGO that works with doctors who volunteer their services. Third, if the outcome of their regular asylum procedure is unsuccessful, persons seeking asylum can still apply for a residence permit on humanitarian grounds. For instance, they may claim to suffer from a serious illness that cannot be treated in the country of origin or which impairs their ability to travel. The regulations cover claims of mental disorders, and include the risk of suicide if asylum is not granted (ECRE, 2020; Meffert et al., 2010).

In 2019, 29.435 persons applied for asylum in the Netherlands (Immigration and Naturalization Services, 2020). After a negative decision, the judicial process underlying these applications allows several possibilities for appeal. A procedure for asylum may therefore take several years (Rijksoverheid, 2019). Meanwhile, the prevalence and severity of mental disorders tends to increase during lengthy procedures. For instance, Laban et al. (2005) found that the original prevalence of $42 \%$ increased to $66.2 \%$ in Iraqite applicants for asylum after a procedure of more than two years. However, an issue with this type of epidemiological data concerning psychiatric morbidity in persons seeking asylum is that it usually relies on data obtained from self-reporting. It is therefore conceivable that some applicants may distort their self-reported symptoms to qualify for humanitarian provisions in the application procedure (Lustig, 2008).

The act of intentional exaggeration or full fabrication of symptoms in order to obtain an external benefit is known as malingering (DSM-5; APA, 2013). In settings where there is an external gain for patients to exaggerate their complaints, Symptom Validity Tests (SVTs) can be used to screen for this response bias (Bush et al., 2015). For example, one type of SVT, the 
Self-Report Validity Test (SRVT) is used to detect exaggerated self-reports by incorporating implausible symptoms that are likely to be endorsed by patients who exhibit overreporting tendencies. In migrants, however, differences in culture and language may confound the outcomes of SVTs (see the review by Nijdam-Jones \& Rosenfeld, 2017). Some SVTs have been translated and appear to function adequately in ethnic groups for which they were not originally designed (e.g., DuAlba \& Scott, 1993; Montes \& Guyton, 2014; Vilar-Lopez et al., 2007). However, in culturally diverse settings, such as referral centers for refugee mental health patients, translated and validated instruments are not always available. Thus, the staff of such centers may try to cope with this situation by employing dialogue interpreters, which was shown to be an error-prone method (Bot, 2005). Another option is to use tests with low verbal mediation (Benuto et al., 2014; Erdodi et al., 2017), such as Performance Validity Tests (PVTs), that focus on the exaggeration of impairment (Dandachi-FitzGerald et al., 2013; Martin et al., 2015).

PVTs consists of tasks with a cognitive load that is light enough to allow patients with genuine symptoms to pass with sufficient effort. This is known as the 'floor effect'. Previous studies have shown that patients who fail on PVTs (i.e., perform below standards) often engage in malingering (Merten \& Merckelbach, 2013; Young, 2014). However, most PVTs that are currently available were devised for use by patients in Western Europe and the United States. In patients with a migration background, the cognitive load needed to induce the floor effect could thus be sensitive to their cultural backgrounds and education. This has led to an ongoing controversy about the intercultural validity of cognitive tests. Lynn and Vanhanen $(2002,2006)$ used methods such as literature reviews and student assessments to construct average IQ estimates for many countries. Their conclusion was that there are substantial differences in average performance on cognitive tests between different regions in the world, ranging from an average 'national IQ' of 108 in Singapore and Hong Kong, to 60-70 in some regions of Sub-Saharan Africa (Lynn \& Meissenberg, 2010). The controversy generated by their findings focused on their methodology, on their correlation with measures of economic prosperity, social well-being, and other possible causes of these differences (Barnett \& Williams, 2004; Hunt, 2011; Rindermann, 2018; Wicherts et al., 2010). In addition, the notion of a possible genetic factor fueled racist agendas and eugenics movements (Cafasso, 2020).

However, if such geographical bias in cognitive test performance actually exists, it could put refugee mental health patients at risk for misclassification by European or American PVTs. More specifically, following the reasoning of Lynn and Vanhanen, refugee mental health 
patients from some regions would be more at risk than others. In the present study, we therefore addressed the following hypothesis: In refugee mental health patients, PVT outcomes are confounded by regional differences.

\section{Method}

\section{Participants}

Our sample consisted of inpatients admitted to a non-forensic psychiatric referral center for refugee mental health in the Netherlands during the study period (2008-2012). This sample included inpatients both with and without refugee status. The inclusion criterion was willingness and ability to give informed consent for participation in the study. More than $95 \%$ of the inpatients were willing and able to give consent. The study was approved by the Central Committee on Research Involving Human Subjects (CCMO).

Sample 1. A total of 203 inpatients of the referral center were included during the study period. Of the total sample, 93 (46\%) inpatients originated from Africa, 49 (24\%) from the Middle East, 40 (20\%) from the former USSR, 13 (6\%) from Eastern Asia, and eight (4\%) from former Yugoslavia. Their mean age was 33.8 years $(S D=11.5)$, and $52(26 \%)$ were female. Independent from the intervention, the patients were classified as follows: 131 patients (65\%) were classified as having a positive incentive to exaggerate, $49(24 \%)$ as having no incentive or mixed incentives to do so, and $23(11 \%)$ as having a negative incentive to exaggerate impairment (i.e., an incentive to minimize or deny impairment). Both the no/mixed and the negative incentive group were regarded as patients without incentives ( see Incentives to Exaggerate Impairment and/or Distort Symptoms).

Patients were offered the option to participate in Dutch language classes in the referral center, during which the level of their proficiency in Dutch was assessed (see below). In total, 167 patients of the study sample participated. Of this total, 89 (53\%) had poor proficiency (level A0), 38 (23\%) intermediate proficiency (level A1), and 40 (24\%) advanced proficiency (level B and above). A number of patients in this sample (17\%) were not alphabetized in Latin script (i.e. they were unable to read or write in the western alphabet).

Sample 2. A subgroup of the participants $(n=49)$ of sample 1 who passed the PVT (i.e., had error scores below the cut-off) on admission were tested for a second time with the same PVT towards the end of their admission. This was done to check the accuracy of our 
assignment into incentive groups (see below). Of these patients, 21 (42.9\%) originated from Africa, 17 (34.7\%) from the Middle East, 7 (14.3\%) from the USSR, and 4 (8.2\%) from Eastern Asia. Their mean age was $43.1(S D=10.3)$, and $35(71.4 \%)$ were men. The classification showed that 21 patients (43\%) in the subgroup had a positive incentive to exaggerate impairment, 18 (37\%) had no or mixed incentives to do so, and 10 (20\%) had a negative incentive (see below).

\section{Measures}

Forced-Choice task. This is a forced-choice PVT involving the identification of basic emotional expressions. It is modelled on the Morel Emotional Numbing Test (MENT; Morel, 1998), which is sensitive to response distortion in the assessment of trauma-related problems. The version used here consisted of 20 colored pictures of 10 facial expressions posed by a man and a woman. Their expressions reflect happiness, frustration, sadness, anger, fear, calmness, surprise, shyness, confusion, and sleepiness (Geraerts et al., 2007). The pictures were presented on a computer screen $(30 \times 38 \mathrm{~cm})$. Before the test, patients were told that emotional numbness is a prominent symptom of trauma-related problems and that this may cause people to have difficulties with the recognition of facial expressions. The rationale behind this instruction was that individuals who want to exaggerate trauma-related symptoms may intentionally produce more errors on this simple test. After this, patients were instructed to identify the emotion that best matched the expression of the face in three runs of 20 trials; one with one picture and two labels, one with two pictures and one label, and the last one with two pictures and two labels; their task was to connect these stimuli in the right way. The emotion labels used in the test were translated and back-translated into several languages. Errors were summed across the three runs. There were no missing data. Cronbach's alpha indicated high internal consistency (0.94).

Morel recommended a cutting score of nine errors on the MENT, with scores above this level raising the suspicion of underperformance. This cut-off point has been validated in a sample of Croatian war veterans on a translated version of our forced-choice task, where it was found to discriminate between treatment-seeking and compensation-seeking veterans (sensitivity = 92\%; specificity = 96\%; Geraerts et al., 2009).

Implausible symptom inventory. This SRVT is the Dutch version of the Structured Inventory of Malingered Symptomatology (SIMS; Widows \& Smith, 2005), which was adapted for use in refugee mental health patients. Like the SIMS, it consists of 75 true-false items that describe atypical and rare symptoms and experiences (e.g., "There is a constant ringing 
in my ear"; "The voices that I hear have never stopped since they began"). There are five subscales, each containing 15 items, which address commonly feigned conditions: amnesia, neurologic impairment, psychosis, affective disorders, and low intelligence. After recoding some items, endorsed symptoms are summed to obtain a total score, with higher scores indicating more symptom over-endorsement. Previous studies recommended a cut-off of 16 for a comprehensive assessment of feigning (Merckelbach \& Smith, 2003). Van Impelen et al. (2014) summarized psychometric data indicating satisfactory internal consistency of the Dutch SIMS (with Cronbach's alpha's ranging from 0.80-0.96), acceptable test-retest stability $(r=0.72-0.97)$, and good ability to discriminate between symptom exaggeration and honest responding (sensitivities varying between $0.75 \%$ and $100 \%$ ).

In the current study, some items of the Dutch SIMS version (Merckelbach et al., 2001) were adapted. For example, after consultation with professional translators, the item pertaining to the queen of Holland (part of the subscale that assays for exaggeration of low intelligence) was rephrased as follows: "The prophet of Allah is called Mohammed" (for patients with a Muslim background) and "The mother of Jesus is called Mary" (for patients with a Christian background). No data were missing. Cronbach's alpha was 0.95 and varied for subscales between low ( 0.69 for affective symptoms subscale) and excellent ( 0.89 for amnesic symptoms subscale).

Proficiency in Dutch. Patients were independently assessed by Dutch language teachers employed at the referral center. Based on fixed criteria (Meijer \& Noijons, 2008), these experts categorized patients into patients with poor proficiency in Dutch ('absolute beginners', level A0), intermediate proficiency ('beginners', level A1), and good proficiency ('advanced students', level B and above). The teachers were blinded to inpatients' test outcomes and their assignment to incentive groups.

Incentives to exaggerate impairment and/or distort symptoms. Social workers of the hospital independently evaluated incentive levels of patients on the basis of file information: they differentiated between the presence of a positive incentive to exaggerate impairment/ symptoms, the absence of an incentive to do so, and the presence of a negative incentive (i.e., an incentive to hide or minimize impairment/symptoms). They did this by adding one point for each condition potentially promoting exaggeration, such as: 1. An asylum procedure still in progress; 2. A temporary refugee status issued for medical reasons; 3 . Any other current procedure necessitating a medical report indicating medical necessity, urgency or exemption (e.g., request for family reunion while the patient is unable to earn the income required by Dutch 
law; a request for urgent change of housing or special housing arrangements; a request to be exempted from the demand to pass the language test in the naturalization procedure, etc.). For each condition discouraging exaggeration they subtracted one point. Such circumstances were: 1 . Compulsory nature of the present admission; 2. Any current procedure necessitating a medical report indicating improved functioning or decreased need for medical treatment or scrutiny (e.g., a child custody procedure, a request for voluntary repatriation, etc.). Patients with one point or more were considered to have a positive incentive, and patients with minus one point or less were considered to have a negative incentive. Patients with zero points were considered to have no incentives, although some actually had a mix of positive and negative incentives. For practical purposes, patients in the negative incentive group and in the no/ mixed incentive group were both assumed to have no (positive) incentive to exaggerate. The social workers were blinded to the test outcomes and the proficiency estimates of the Dutch language teachers.

\section{Procedure}

To test whether PVTs designed for American and European patients are sensitive to geographical differences when used in refugee mental health patients, we compared the test performance of inpatients at a referral center for refugee mental health in the Netherlands from five regions: 'Sub-Saharan Africa', 'the Middle East', 'Former USSR', 'Eastern Asia', and 'Former Yugoslavia'. According to Lynn and Meissenberg (2010) the average IQ of the nations in these regions is $60-75$ for Sub-Saharan Africa, 80 - 85 for the Middle East, $90-100$ for the former USSR, 85 - 105 for the Eastern Asia, and 90 - 100 for former Yugoslavia.

For a valid comparison, we also studied possible associations of test outcomes with two confounders: poor proficiency in Dutch and the presence of external incentives to distort symptoms and/or exaggerate impairment. Although we selected a PVT with low verbal mediation that was available in several languages, its presentation still needed to be assisted by a staff member and a dialogue interpreter, as some of the patients ( $n=28 ; 17 \%$ ) reported that they were illiterate. To account for the confounding effects of this suboptimal method of presentation, we also studied the scores of the participating patients on a SRVT with high verbal mediation and compared outcomes of both tests to the individual levels of proficiency in Dutch of the patients. The SRVT interviews were also assisted by a staff member an a dialogue interpreter. Patients with a poor proficiency were expected to earn higher scores, especially on the high verbal mediation SRVT. An impression of the strength of such an association was 
considered important, as a large effect could interfere with our comparison between regions.

Another confounder that could possibly affect our comparison between regions is that some patients' might be inclined to exaggerate or feign their complaints. To account for this, we looked for a possible association of test outcomes with such incentives. Thus, we divided the patients into a group with external incentives to exaggerate and a group without such incentives. The presence of incentives was coded using available corroborative information, and was not based on patients' self-report.

To ensure that our assignment of patients to incentive groups was accurate, we ran additional checks. First, the PVT we selected presented the same items throughout the test in various combinations. We checked whether there would be a learning effect towards the end of the test in patients who were assigned to the group without incentives. Such a learning effect would be regarded as an indication of correct classification. Second, a subgroup of the patients who passed the PVT (i.e., had error scores below the cut-off) at admission were given the same test again later towards the end of their admission. Patients assigned to the no incentive group passing the test a second time would also be taken to confirm the accuracy of our selection method.

Staff members who assisted with test instructions were blinded to the information obtained by the social workers and the proficiency estimates of the Dutch language teachers. However, they were involved in the treatment of the patients and had access to their medical files, so blinding was less than complete.

\section{Data analysis}

We used descriptive statistics to evaluate the test outcomes. As our data were skewed or unevenly distributed (Shapiro-Wilk test: $W=0.908, p<.001$ and $W=0.947, p<$ 0.01 , respectively), we used Kruskal-Wallis and Mann-Whitney $U$ tests to compare groups and Spearman's rho for correlations.

\section{Results}

\section{General scores on PVT and SRVT}

The mean error score on the administered PVT was 18.7, 95\% Cl $[16.7,20.7]$ with a range of 0 to 54, while 123 patients (61\%) had error scores above the cut-off (> 9 errors); 54 
patients (27\%) failed on more than half of the items. On the SRVT, the implausible symptom inventory, the mean score was 33.8, 95\% Cl [31.5, 36.1] with a range of 3 to 62, while 164 patients (81\%) scored above the cut-off. The error scores on the forced-choice task correlated significantly with the scores on the implausible symptom inventory (Spearman's rho $=0.571, p<$ 0.001). All patients who failed the forced-choice task also over-endorsed implausible symptoms.

\section{Language proficiency and PVT and SRVT results}

Patients with poor proficiency made more errors on the forced-choice task, but they did not endorse more implausible symptoms than patients with better proficiency, except for the symptoms in the subscale that screens for exaggeration of intellectual impairment. These findings are summarized in Table 1.

Table 1. Error scores and numbers of endorsed implausible symptoms for each level of proficiency in the language of the host country (Dutch). The forced-choice task has low verbal mediation, whereas the implausible symptom inventory has high verbal mediation. The 'low intelligence' subscale of the implausible symptom inventory screens for exaggeration of intellectual impairment.

\begin{tabular}{lcccc}
\hline & $\begin{array}{c}\text { Poor } \\
\text { proficiency } \\
n=89\end{array}$ & $\begin{array}{c}\text { Intermediate } \\
\text { proficiency } \\
n=38\end{array}$ & $\begin{array}{c}\text { Good } \\
\text { proficiency } \\
n=40\end{array}$ & $X^{2}$ \\
& Mean (SD) & Mean (SD) & Mean (SD) & \\
\hline $\begin{array}{l}\text { Forced-choice task } \\
\text { Number of errors (cut-off }>\text { 9) }\end{array}$ & $22.6(15.4)$ & $15.2(11.8)$ & $14.2(13.5)$ & $12.376^{* *}$ \\
$\begin{array}{l}\text { Implausible symptom inventory } \\
\text { Number of endorsed items (cut-off }>16)\end{array}$ & $36.7(16.7)$ & $34.9(16.1)$ & $32.9(15.9)$ & 1.942 \\
$\begin{array}{l}\text { Implausible symptom Inventory- LI } \\
\text { Subscale 'low intelligence' }\end{array}$ & $5.5(3.5)$ & $4.4(2.7)$ & $3.5(2.9)$ & $10.558^{* *}$ \\
\hline
\end{tabular}

${ }^{*} p<0.05 ;{ }^{* *} p<0.01$

\section{External incentives and PVT and SRVT results}

Patients with a positive incentive to exaggerate made more errors on the forced-choice task and endorsed more implausible symptoms than the mixed and negative incentive group. However, the scores of substantial proportions of the mixed and negative incentive group also exceeded the cut-off of both the forced-choice task and the implausible symptom inventory. Patients failed the implausible symptom inventory more often than the forced choice task; 
$96 \%$ vs. $79 \%$ in the positive incentive group, $63 \%$ vs. $29 \%$ in the no/mixed incentives group, and $30 \%$ vs. $22 \%$ in the negative incentive group. For a summary of the findings, see Table 2 .

Table 2. The scores of refugee mental health patients on the forced-choice task and the implausible symptom inventory. The patients are divided in groups with either a negative incentive to exaggerate impairment (i.e., an incentive to minimize impairment), with no/mixed incentives to do so, or a positive incentive to exaggerate.

\begin{tabular}{lcccc}
\hline & $\begin{array}{c}\text { Negative } \\
\text { incentive } \\
n=23\end{array}$ & $\begin{array}{c}\text { No/mixed } \\
\text { incentives } \\
n=49\end{array}$ & $\begin{array}{c}\text { Positive } \\
\text { incentive } \\
n=131\end{array}$ & $X^{2}$ \\
& Mean (SD) & Mean (SD) & Mean (SD) & \\
\hline $\begin{array}{l}\text { Forced-choice task } \\
\text { (Number of errors) }\end{array}$ & $6.4(3.7)$ & $8.0(7.3)$ & $24.9(14.3)$ & $69.142^{* *}$ \\
$\begin{array}{l}\text { Implausible symptom inventory } \\
\text { (Number of endorsed items) }\end{array}$ & $16.6(11.9)$ & $23.1(14.8)$ & $40.8(13.3)$ & $64.425^{* *}$ \\
\hline
\end{tabular}

${ }^{*} p<0.05 ;{ }^{* *} p<0.01$

\section{Learning effect during the PVT}

The patients in the negative and the mixed/no incentive groups made on average fewer errors towards the end of the test (i.e. in the last set of 20 items). The average error score of patients with a positive incentive to exaggerate remained stable throughout the test. For a summary, see Table 3.

Table 3. Mean error scores on each consecutive set of the forced-choice task. The patients are divided in groups with either a negative incentive to exaggerate impairment (i.e., an incentive to minimize impairment), with no/mixed incentives to do so, or a positive incentive to exaggerate.

\begin{tabular}{lccc}
\hline & $\begin{array}{c}\text { Negative incentive } \\
(n=23)\end{array}$ & $\begin{array}{c}\text { No/mixed incentives } \\
(n=49)\end{array}$ & $\begin{array}{c}\text { Positive incentive } \\
(n=131)\end{array}$ \\
\hline $\begin{array}{l}\text { First set } \\
\text { Mean }(S D)\end{array}$ & $2.9(1.8)$ & $3.2(2.6)$ & $8.8(5.0)$ \\
$\begin{array}{l}\text { Second set } \\
\text { Mean }(S D)\end{array}$ & $3.0(2.6)$ & $8.0(4.7)$ \\
Third set & $2.0(1.3)$ & $1.9(2.6)$ & $8.1(5.6)$ \\
Mean $(S D)$ & $1.1(1.3)$ & & \\
\hline
\end{tabular}




\section{Learning effect in between PVTs}

The average period between tests in this subsample was 9.7 weeks $(S D=8.8)$. Patients with a negative incentive to exaggerate $(n=10)$ or no/mixed incentives to do so $(n=18)$ who passed the test the first time also passed it the second time. Patients with a positive incentive to exaggerate $(n=21)$ who passed the test the first time made on average more errors the second time, resulting in test failure in the majority. For an illustration and a summary of the results, see Figure 1.

Figure 1. Error scores on the forced-choice task for patients with a negative incentive to exaggerate impairment (i.e., an incentive to minimize impairment), patients with no/mixed incentives and patients with a positive incentive to exaggerate impairment. The forced-choice test was presented twice: the first time at admission ("forcedchoice1"), and the second time towards the end of the admission ("forcedchoice2").

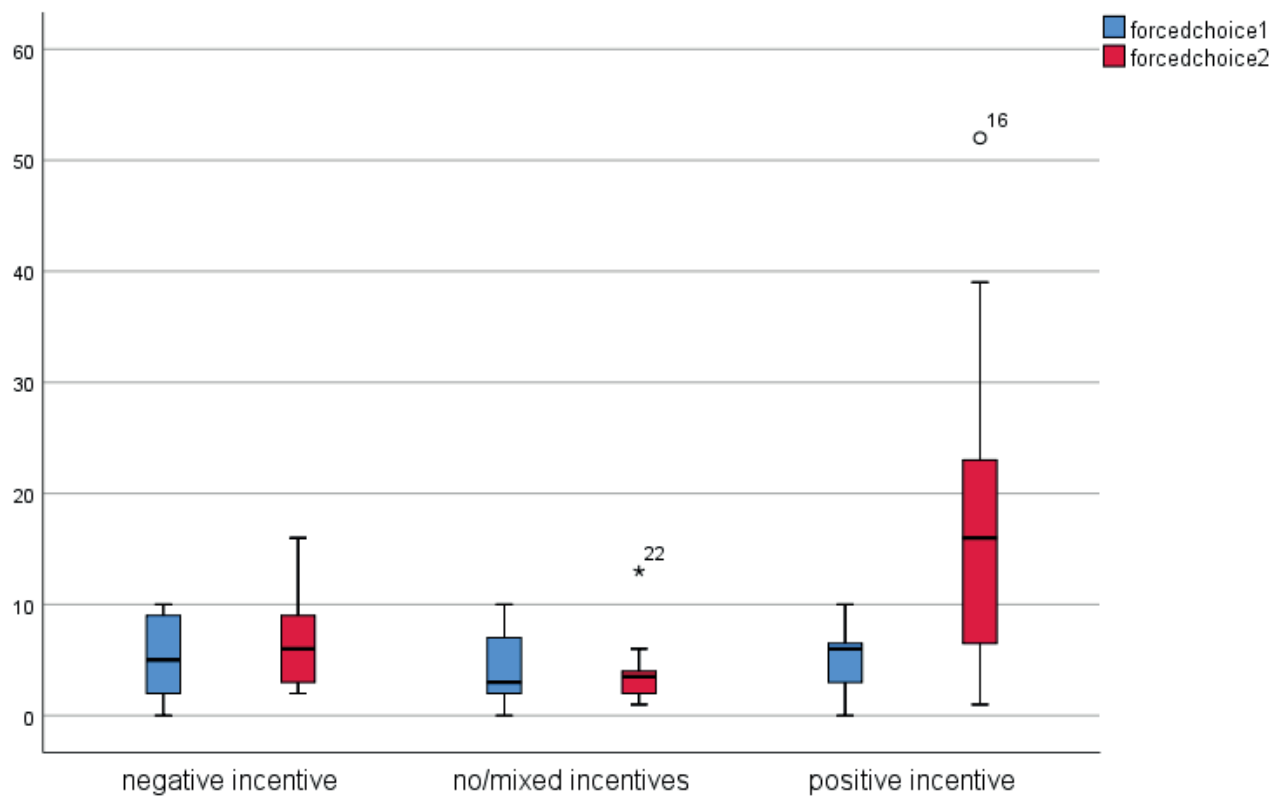

\section{PVT and SRVT results between regions}

Without accounting for linguistic factors and external incentives, the differences in average error scores between regions of origin were significant $\left(\chi^{2}(4)=13.96, p<0.01, \eta^{2}=\right.$ 0.05). The outcomes ranged from an average of 23.1 errors $(S D=15.6)$ for inpatients from the former USSR to an average of 7.2 errors $(S D=7.6)$ for those from the former Yugoslavia. For a summary, see Table 4. 
Table 4. Error scores and numbers of endorsed items of refugee mental health patients on the forced-choice task and the implausible symptom inventory, per region of origin, before controlling for incentives to exaggerate impairment/symptoms.

\begin{tabular}{|c|c|c|c|c|c|c|}
\hline & $\begin{array}{l}\text { Africa } \\
n=94\end{array}$ & $\begin{array}{l}\text { Middle East } \\
\quad n=49\end{array}$ & $\begin{array}{l}\text { Former USSR } \\
\qquad n=40\end{array}$ & $\begin{array}{c}\text { Far East } \\
n=13\end{array}$ & $\begin{array}{c}\text { Former } \\
\text { Yugoslavia } \\
n=8\end{array}$ & $x^{2}$ \\
\hline & Mean (SD) & Mean $(S D)$ & Mean (SD) & Mean $(S D)$ & Mean $(S D)$ & \\
\hline $\begin{array}{l}\text { Forced-choice task } \\
\text { (Number of errors) }\end{array}$ & $20.3(14.7)$ & $14.4(13.2)$ & $23.1(15.5)$ & $16.8(14.0)$ & $7.2(7.6)$ & $13.955^{*}$ \\
\hline $\begin{array}{l}\text { Implausible symptom } \\
\text { inventory } \\
\text { (Number of endorsed items) }\end{array}$ & $35.6(16.2)$ & 0.3 (16.7) & $39.3(15.3)$ & 24.9 (17.7) & $3(11.5)$ & 15. \\
\hline
\end{tabular}

${ }^{*} p<0.05 ;{ }^{* *} p<0.01$

Although patients with poor proficiency made on average more errors than patients with good proficiency, the differences between proficiency groups were not significant when only the error scores of patients without an incentive to exaggerate were selected for this comparison $\left(\chi^{2}(2)=5.84, p=0.054\right)$.

If only patients without incentive to exaggerate impairment were selected for comparison of the average error scores between regions of origin, the differences were not significant. For a summary, see Table 5.

Table 5. Error scores and endorsed items of refugee mental health patients on the forced-choice task and the implausible symptom inventory, per region of origin, after controlling for incentives (only patients without incentive to exaggerate impairment were included).

$\begin{array}{cccccc}\text { Africa } & \text { Middle East } & \text { Former USSR } & \text { Far East } & \text { Former } & \\ (n=32) & (n=19) & (n=7) & (n=8) & \text { Yugoslavia } & X^{2} \\ & & & & (n=6) & \end{array}$

Mean (SD) Mean (SD) Mean (SD) Mean (SD) Mean (SD)

Forced-choice test

(Number of errors)

$8.5(7.3) \quad 5.5(2.3)$

$7.7(7.8)$

$9.4(8.1)$

$5.7(5.5)$

2.576

Implausible symptom

inventory

$22.3(16.0) \quad 20.4(12.6) \quad 23.6(18.1)$

$15.9(6.7) \quad 20.5(13.5)$

0.452

(Number of endorsed items)

${ }^{*} p<0.05 ;{ }^{* *} p<0.01$ 


\section{Discussion}

After considering the debate about possible regional differences in the world related to average performance on cognitive tests that were originally devised for American and European patients, we determined whether there are significant differences in average PVT performance between refugee mental health patients from these regions. We assumed that the cognitive load presented to generate the floor effect by the PVT was excessive or some patients, depending on their region of origin, resulting in significant differences in average group scores. Although we did find significant differences in average scores between regional groups, these were not in line with expectations based on the findings of Lynn and Meissenberg (2010). We found that patients from the former USSR had the highest average error score, even though this region did not have the lowest average "national IQ". In addition, when we controlled for external incentives by only selecting patients for the comparison without incentive to exaggerate, the differences between regional groups were no longer significant. After we controlled for external incentives, our results did not support the hypothesis that the floor effect strategy of PVTs is confounded by regional differences.

In our study we accounted for two other confounders that might disrupt the PVT performance of participants. First, we checked whether the performance of the patients in our sample was associated with their proficiency in Dutch, where poor proficiency predicted poor performance. As expected, we found that patients failed the SRVT - which has high verbal mediation - more often than the PVT - which has low verbal mediation. This appears to confirm that linguistic factors act as a confounder. This finding is also in line with earlier research on this topic. Erdodi et al. (2017) found increased failure rates in subjects with limited English proficiency on PVTs with high verbal mediation in comparison to PVTs with low verbal mediation.

However, we did not find the association with proficiency that we expected. Patients with poor proficiency indeed made more errors on the low verbal mediation PVT, but they did not earn higher scores on the high verbal mediation SRVT. Patients with poor proficiency endorsed more implausible symptoms only if these screened for exaggeration of low intelligence. This suggests that the Dutch language teachers may have underestimated the proficiency of patients, some of whom might have been feigning intellectual impairment. We therefore repeated the comparison between proficiency groups, but this time we only included patients assigned to the negative and no/mixed incentive groups. As this eliminated group differences, 
we concluded that lack of proficiency in Dutch was not a major confounder in our sample. However, this does not exclude the possibility that differences in language and culture are confounding factors. For example, in a survey conducted in 2014, a large majority of emotion researchers still endorsed the notion that facial expressions are recognized universally by humans (Ekman, 2016), even though this idea has been challenged in recent years by studies reporting that members of some culturally isolated groups assign other emotions to certain facial expressions (Crivelli et al., 2016; Gendron et al., 2014).

Surprisingly, we found that the PVT and SRVT outcomes were correlated. As PVTs and SRVTs tap into different dimensions of symptom distortion (exaggeration of impairment vs. self-reported symptoms), this was unexpected and not in line with previous research in mixed-psychiatric samples (Dandachi-FitzGerald et al., 2011; Dandachi-FitzGerald et al., 2016). The association we found suggests that there is a common factor in the study sample that differentiates it from other mixed-psychiatric samples. For example, one major factor that is shared by most refugee mental health patients, including the patients in our sample, is a history of psychological trauma. When the MENT and our forced-choice task are introduced to the patients, impaired emotion recognition is attributed to psychological trauma. In addition, Van der Heide et al. (2017) found high scores on the Harvard Trauma Questionnaire (HTQ; Mollica et al., 1992) in a sample of refugee mental health patients that were associated with over-endorsement on the SIMS. The refugee mental health patients in our sample could have linked both impaired emotion recognition and high symptom scores to psychological trauma. Another option is that these patients resorted to distortion in more than one dimension of symptom distortion because of the catastrophic effects of a failed request for asylum.

Considering that we found a robust association between test performance and presence of external incentives, this explanation is tenable. This suggests that external incentives were a major confounder in our study sample, rendering the test results of patients in the positive incentive group of little value for our comparison between regions. However, our division into incentive groups was inherently crude. We only used objectifiable file information in the assignment process; we disregarded subjective (internal) incentives to distort symptoms, such as previous experiences of having been denied access to care, or that such distortions were a cry for help. Nevertheless, patients without external incentives may still be motivated by such internal incentives to misrepresent symptoms and/or impairment.

For this reason, we conducted an additional check of participants' assignment to incentive groups. Its accuracy was confirmed in two ways: patients without external incentives 
to exaggerate were inclined to make fewer errors in the repetitive PVT task towards the end of the test, and in a subsample, they also passed the PVT the second time it was presented to them. Both approaches clearly differentiated patients without external incentives from those with such incentives.

In the entire study sample, more than half of the patients failed two different types of SVT. This is an exceptionally high proportion. Could this lead to the additional conclusion that some of the patients were actually malingering? Although there some findings in our study and previous research appear to support such conclusion, the answer to this question is not straightforward.

First, more than half of the patients failed two tests that tap into different domains of illness distortion. According to Larrabee (2008) the likelihood ratios of multiple tests can be chained, increasing the post-test probability of malingering. On the other hand, Berthelson et al. (2013) contended that the false positive rate may also increase when multiple tests are used, and in their re-calculation of Larrabee's data, Bender and Frederick (2018) found a smaller effect than Larrabee by considering both negative and positive trials (Larrabee: posttest probability of feigning 0.936 to 0.973 after two trials; Bender and Frederick: 0.569 to 0.865). They argued that "It seems reasonable to conclude that the use of multiple feigning tests sharply increases the possibility of false-positive classification of individuals who are compliant - not just when the tests are correlated - but because the false-positive error rate accumulates with the administration of each additional validity measure" (Bender \& Frederick, 2018). In this view, the use of multiple tests in the referral center may have contributed to the relatively high failure rates, including those in the negative incentive group.

Second, more than a quarter of the patients failed more than half of the items of the binary forced-choice task. According to some authors, this type of below-chance performance is a strong indication of malingering. However, others contend that below chance should be defined as $p<0.05$, not $p<0.50$ (for an overview, see Young, 2014). In our sample only a small number of patients attained scores in the significantly below-chance range based on binomial probability theory.

Third, the patients of the positive incentive group failed to make less errors towards the end of the forced-choice task that essentially presented the same task throughout the test. This absence of learning effect can be regarded as a form of response-curve detection strategy. Although such an additional detection strategy may add to the strength of a PVT, it does not reduce the limitations of a differential prevalence design as far as prevalence 
estimates are concerned (see below).

Fourth, in the second sample, patients with a positive incentive failed the same PVT they passed earlier at the start of their admission. This reverse learning effect in between tests looks like a convincing indicator, but follow-up PVTs were only presented to a minority of the study sample that may not have been representative. This was because only patients who passed the forced-choice task at admission were included in this particular subsample.

Fifth, SVT failure is not enough to classify the behavior of a patient as malingering. Instead, a multi-modal approach is advocated ("Slick criteria"; Slick et al., 1999; Sherman et al., 2020) of which symptom validity tests are only one part.

And finally, the association of the test outcomes with external incentives was strong, and our division into incentive groups appears to have been reasonably accurate. However, the method we used - dividing patients into groups based on presumed incentives, also known as the differential prevalence design - cannot estimate the prevalence of malingering in a sample (Rogers, 2018). To make such an estimation, known-groups comparisons, bootstrapping techniques or experimental simulations are used. To construct known groups, however, knowledge about base rates (of malingering) and dependable experts for case selection (of malingerers) are needed. For bootstrapping, SVTs that are validated for the target group are required. At best, the technique we used to check the validity of our method to assign incentive groups could be used for future simulation studies in refugee mental health patients. At present, symptom validity in refugee mental health patients is still insufficiently studied to make inferences about the prevalence of malingering in this population.

Another important limitation of our study is that we only accounted for two possible confounders. As mentioned above, the fact that this study failed to highlight regional differences between patients does not mean that PVTs are unaffected by cultural factors (e.g., different test taking attitudes; Ardilla, 2005). Other confounders of PVTs have also not been ruled out in our study, such as intellectual disability (Shandera et al., 2010), alexithymia (Merckelbach et al., 2018), psychotic symptoms (Schroeder \& Marshall, 2011; Van der Heide \& Merckelbach, 2019), or careless responding (Meyer et al., 2013). Even though we found no regional differences after controlling for external incentives as a factor, the presence of one confounder does not necessarily rule out the presence of another (Merten, 2017). This is why PVT failure is relatively common in mixed-psychiatric populations. Mcwhirter et al. (2020) reviewed PVT studies in clinical populations with psychiatric disorders, intellectual disability, degenerative brain disease, brain injury, functional disorders and epilepsy and found failure rates for some 
groups and tests exceeding $25 \%$. In our sample we found similar rates in the patient groups without incentives.

Finally, the patients we studied were part of a highly selected, clinical group; for this reason our findings cannot be generalized to larger populations of refugee mental health patients. In terms of future research, however, one important finding of our study is that symptom validity can be very poor in refugee mental health patients. This has implications for future epidemiological research into the prevalence of psychiatric morbidity in this target group. For example, the findings of Laban et al. (2005), which were discussed in the introductory section of this article, were not checked for symptom validity. As suggested by those authors, it is quite conceivable that the stress generated by lengthy procedures for asylum caused the increase in morbidity in their study. Yet, our present findings indicate that a change in test outcomes in time may also be an element of adaptive behavior. One way to rule this out is to include SVTs in the design of future prevalence studies in refugee mental health.

\section{Conclusion}

The data gathered in this study reject our hypothesis that PVT outcomes in refugee mental health patients are confounded by regional differences in the cognitive load that is required for patients with genuine impairments to pass a PVT with sufficient effort. 



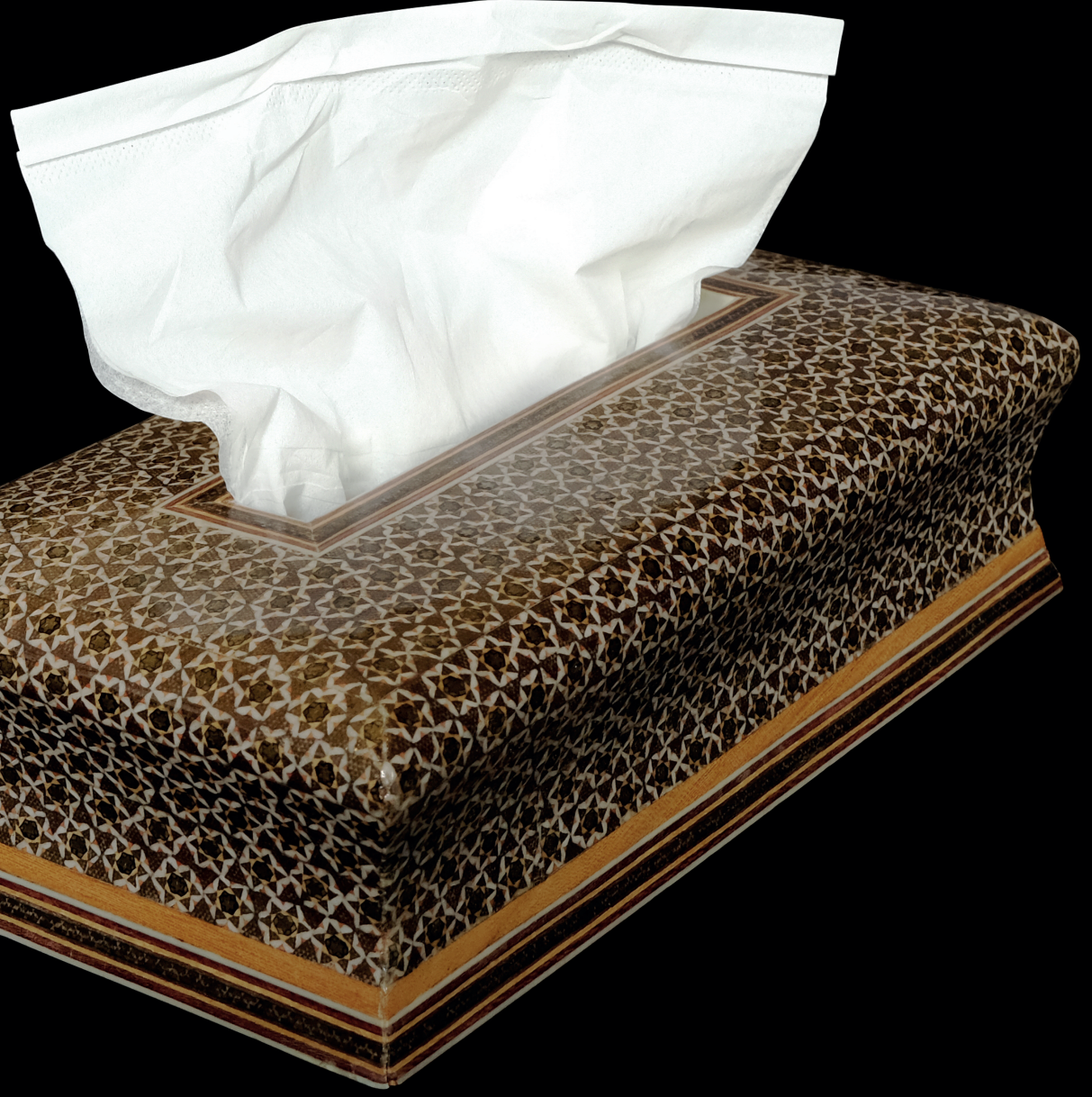




\section{0}

GENERAL DISCUSSION 


\section{Introduction}

Many articles on mental health problems in refugees and persons seeking asylum have shown that this population has an increased risk of serious psychopathology such as PTSD and depression (e.g., Mollica et al., 2007), but also psychosis (e.g., Hollander et al., 2017). Professionals involved with refugee mental health therefore tend to highlight the vulnerability of this target group and hence the need for additional care provisions (WPA, 2017). These professionals emphasize, for good reasons, that their patients managed to survive extreme adversarial conditions at home, endured a great deal of hardship and danger while in transit, and after reaching their destinations, were finally able to be referred to specialist mental health services - despite the scarcity of such services for persons seeking asylum in increasingly xenophobic host countries (e.g., Loutan et al., 1999; Siman-Tov et al., 2019). In this light, it is also understandable that the issue of symptom feigning among refugees and persons seeking asylum has not received much attention, even though acquiring a legal permission to stay in the host country could be an incentive for some of the them to feign a mental condition. When confronted with threats of repatriation to a dangerous country of origin, feigning can even be said to be a survival strategy (see also, Lustig, 2008; Meffert et al., 2010). At the same time, concepts such as feigning and malingering are strongly associated with negative moral overtones (but see Beach et al., 2017). Therefore it is legitimate to question whether feigning should be examined in the context of refugees and persons seeking asylum, groups that are arguably vulnerable and in a powerless social position. One reason for doing so is that undetected feigning may entail iatrogenic risks for individuals who engage in such feigning. As the case report in the introductory section of this thesis illustrates, information that should heighten clinicians' suspicion of possible feigning tends to be overlooked or even ignored, which can have consequences the person who presents as a patient. Indeed, multiple case reports have been published on the dire consequences of treating feigned symptoms as though they were genuine symptoms (Carneiro et al., 2019; Waite \& Geddes, 2006).

Clinicians may have good reasons to disregard the possibility of feigning. Before they can conclude that a patient is feigning, a wide range of alternative diagnoses must be ruled out. A false accusation of feigning may stigmatize the patient and result in withholding of appropriate care. In persons seeking asylum, failure to obtain a refugee status on medical grounds may result in socio-economic marginalization or possibly extradition. Clinicians may fear litigation or even physical assault by patients and/or their relatives when they bring up the 
possibility of feigning (Resnick et al., 2018). Also, clinicians may not want to contribute to the highly polarized debate regarding migration, because they fear that feigning will be associated with fraud by refugee mental health patients. And finally, they may act out of concern: "I gave the patient a diagnosis of PTSD because he really needed the money" (Morel, 2010, p. 34). Unfortunately, a psychiatric diagnosis also exposes a patient to social stigma and to psychiatric treatments with potentially harmful side effects.

Given this complexity, when clinicians do set out to investigate the possibility of feigning, their priority is to minimize the risk of false classification. Slick et al. (1999) described a multi-method approach for this purpose that has been revised several times in recent years (Slick \& Sherman, 2012; Young, 2014; see, for a review and update of the criteria: Sherman et al., 2020). Part of this approach involves the use of Symptom Validity Tests (SVTs) to assess the validity of self-reported symptoms and impairments (Bush et al., 2005; Heilbronner et al., 2009). Although mounting evidence has shown that such tests may be effectively used for the detection of feigning in non-forensic mixed psychiatric samples (Dandachi-FitzGerald et al., 2011; Dandachi-FitzGerald et al., 2016), until recently no studies have included patients in refugee mental health. In fact, several authors suggested that the utility of current SVTs varies in different populations and is still limited in intercultural settings (Correa, 2018; Nijdam-Jones \& Rosenfeld, 2017). An important consideration is that accurately translated and validated instruments are often unavailable for culturally diverse populations like refugee mental health patients. In such circumstances, health professionals may resort to live translation by interpreters, which is an error-prone method (Bot, 2005; Correa, 2018). Alternatively, clinicians in these circumstances may rely on their intuition, but clinical intuition has been shown to have a poor track record when it comes to the accurate detection of feigning. For example, Heaton et al. (1978) provided neuropsychologists with clinical test scores of 16 instructed feigners and 16 head-injured patients. The neuropsychologists had to determine for each set of test scores whether it belonged to a genuinely impaired patient or to an instructed feigner. Classification accuracy was generally poor (i.e., detection ranged from chance level to $20 \%$ above chance level). Similarly, a review of 12 studies investigating the ability of medical experts to distinguish between feigned and genuine self-reported symptoms in a clinical consultation found the detection rate of feigning to range between 0 and 25\% (Rosen \& Philips, 2004). More recently, Dandachi-FitzGerald et al. (2017) compared clinical impressions of neuropsychologists and their patients' performance on SVTs and Performance Validity Tests (PVTs). Of the 152 patients for whom neuropsychologists had predicted plausible symptom presentations, 14 patients (9.2\%) 
failed on both instruments. Of the 51 patients for whom neuropsychologists had predicted problematic symptom validity, 35 patients (68.6\%) passed both instruments, indicating the high risk of false positive classifications when experts rely on their intuition.

Given that diagnosis and treatment both require accurate assessment of symptoms, there is a need for research exploring symptom validity in the clinical practice of refugee mental health. In my thesis research I therefore addressed the following research questions:

\section{Is clinical observation, even when it is done during a protracted period of admission in a specialized center, sufficient to rule out illness distortion?}

Some mental health clinicians argue that admission and clinical observation are an effective method to rule out feigning (Conroy \& Kwartner, 2006). However, humans in general may tend to overestimate their cognitive ability in relation to others ("Lake Wobegon" effect; Colman, 2014). If that is the case, then health professionals are no exception. They tend to assume that clinical skills and experience are helpful in discerning feigning behavior (Jelicic et al., 2018), but their patients prove them wrong. A case in point are the "Munchhausen" patients, who time and again lure their physicians into diagnosing life-threatening conditions, followed by major - unnecessary and harmful - surgery (Feldman \& Yates, 2018). Mental health professionals make similar mistakes (factitious mental disorder; Yates et al., 2018).

Patients who are prepared to feign so they can undergo major surgery are usually assumed to be "internally" motivated, for example, to acquire attention and care in a hospital setting. In the absence of an external incentive, this is classified as factitious disorder (DSM 5; APA, 2013). Refugee mental health patients often do have an external incentive, for example, because they need a residence permit for themselves or for their relatives. In such cases, feigning would be classified as malingering. Patients with an external incentive are assumed to take a more rational stance towards their feigning behavior: they take fewer risks and abandon their symptoms as soon as their external goal is achieved (Feldman, 2004; Yates et al. 2018).

Chapter 2 describes two patients in a referral center for refugee mental health in the Netherlands without evidence of motivation other than an external incentive. Yet, both were willing to sustain protracted periods of admission and serious side-effects of specialized treatments that were, in hindsight, unnecessary. Moreover, they presented with psychoticlike symptoms, which has been shown to be unlikely in malingering: "...compared to PTSD, psychosis is infrequently malingered after personal injury. While fraudulent plaintiffs are willing to invest time and energy into crafting a believable story, few are willing to undergo inpatient 
hospitalization and treatment with potent medications" (Resnick et al., 2018).

That the two patients described in Chapter 2 feigned symptoms was discovered accidentally after months of clinical observation. Similar cases of incontrovertible feigning were detected in no more than $1 \%$ of the patients who were admitted to the same referral center during the four-year study period (2008 - 2012). Compared with base rates of feigning behavior in other patient groups with external incentives, this rate is low, even if conservative estimates are used. Estimates tend to vary due to definitional and conceptual ambiguities. In the frequently cited study by Mittenberg et al. (2002), the base rate of feigning was estimated to be $38.5 \%$, but a comprehensive review of base rates by Young (2015) reported a rate of $10 \%$ to $20 \%$ for forensic contexts.

During the four-year study at this referral center, more than half of the admitted patients failed on two or more tests that screen for symptom credibility, with external incentives as the main predictive factor (Chapters 4, 6, 7, 8 and 9). These tests entailed both a screening procedure to evaluate the validity of self-reported symptoms (a Self-Report Validity Test, SRVT) and tests to screen the validity of performance on a cognitive task (a Performance Validity Test, PVT).

Interestingly, a report submitted to the Central Committee on Research Involving Human Subjects (CCMO) at the end of the study period (2008 - 2012) in the referral center (Van der Heide, 2012) also mentioned the poor validity of observational data. Ratings of symptom presence and/or severity obtained by interviewers and nurses based on their observations of patients behavior in the consultation rooms and on the wards were found to correlate to both SRVTs and PVTs. This suggests that the observational data had questionable validity. The numbers of patients included for these comparisons were small, however (e.g., $n=13$ for the rating scale for nurses).

Nevertheless, as discussed later in this chapter, our case study observations do provide evidence that the staff of the referral center failed to detect other feigning patients as well. The answer to the first research question raised in this thesis is therefore that it is highly unlikely that clinical observation is sufficient to rule out illness distortion.

\section{Are there special risks when clinicians rely on their clinical impression in the case of patients with a different cultural background?}

A recurrent theme in Malcolm Gladwell's (2019) bestselling book "Talking to Strangers" is the illusion of transparency, i.e. the illusion that we - laypeople and experts alike - just have 
to look at strangers to make sense of their motives, illnesses, ideals and so on. This illusion will occasionally lead us astray, and this - as Gladwell shows in his book - may become particularly urgent when strangers come from a cultural background that is unfamiliar to us. The vignette in this thesis about the khat-using patient is a case in point. Although it appears to be remote from the overall topic (feigning) of this thesis, this report, together with the vignettes described in Chapter 1 and Chapter 2, illustrates how refugee mental health patients are at risk of suffering serious negative consequences of psychiatric treatments. This is especially the case if their clinicians believe they have to resort to advanced treatment regimens because their symptoms do not respond to more regular, and less hazardous, therapies. In refugee mental health, this is not an unlikely course of events; patients who exaggerate symptoms often need to persist in their behavior for protracted periods of time. A procedure to obtain a refugee status, for example, may take several years (Rijksoverheid, 2019).

The chapter on the khat-using patient addresses a risk that is germane to ethnopsychopharmacology, and therefore to refugee mental health: interactions between psychiatric medication and traditional herbal substances (Bhandari \& Dutta, 2018). The treatment interventions described in this case report clearly went beyond the transparency illusion because they were partly based on 'hard data' - cerebral scans, spinal taps, and biochemical interactions between MAO-inhibitors and other amphetamine-like substances (Ricken et al., 2017; Sjöqvist,1965). However, self-reported data did play an important role when considering treatment options. Some of these self-reports were hard to check; the symptoms of the hypertensive crisis had already subsided upon the patient's arrival at the emergency services, and his concomitant use of medication and substances could not be quantified because MAOinhibitors and khat, both amphetamine-like substances, interact in a laboratory analysis. The conclusion was that use of khat may promote a cerebrovascular accident in a patient who is administered tranylcypromine, even if low dosages of khat seem harmless. Yet, there is a caveat here. Much depends on the credibility of what the patient tells about his khat use. The patient in that case study claimed to use khat in low dosages. Clinicians - as everyone else - tend to default to the truth (Gladwell, 2019), which is a sympathetic starting point. But the question is whether this defaulting to the truth - accepting self-reports of drug use at face-value - is advisable in the case of refugees and persons seeking asylum. This is precisely why this report is included in the current thesis: risks associated with placing too much trust in self-reports of refugee mental health patients can be avoided. One way this can be done is to administer symptom validity tests (note that there are specific symptom validity tests 
to evaluate the self-reports of drug users; Stein et al., 2018). Even though symptom validity tests are only part of the multi-modal approach that is needed to assess self-reports about symptoms and drug use, by themselves they do suffice to substantiate doubts about the validity of self-reported symptoms, regardless of their cause. As discussed in the subsequent sections of this chapter, when symptom validity tests indicate poor validity, the ethical decision is to abstain from high-risk treatments.

\section{Are severe dissociative symptoms reported by refugee mental health patients associated with higher scores on SVTs?}

Apart from relying on their clinical experience and impressions, clinicians may conduct psychological assessments to clarify the needs of their patients and to plan adequate treatment. But information obtained this way is only useful to the extent that patients report symptoms accurately. The accuracy of self-reported symptoms can be explored by relating the outcomes of standard clinical inventories that intend to gauge real symptoms to outcomes of inventories of rare or implausible symptoms (Self-Report Validity Tests, SRVTs). If patients are inclined to endorse both genuine and unlikely symptoms alike, the validity of their self-reported symptoms is poor. However, if standard symptom inventories also include implausible items, endorsement of such items should statistically predict raised SRVT scores.

For example, dissociative experiences are linked to endorsement of bizarre and non-existent symptoms, i.e., symptom over-reporting (for a review, see Merckelbach et al., 2017). Chapter 5 describes a student sample in which participants endorsing a peculiar depersonalization symptom, item 11 of the Dissociative Experiences Scale (DES; Bernstein \& Putnam, 1986), were more inclined to endorse rare or unlikely symptoms listed in Wildman's checklist (Wildman \& Wildman, 1999). This finding is in line with previous research demonstrating that endorsement rates of dissociative symptoms are relatively high in student samples (Merckelbach et al., 2015). Other research on student samples has indicated that this may be due to inattentive and careless responding induced by being involved in lengthy or boring test procedures as a research participant (Meade \& Craig, 2012; Meyer et al., 2013).

In Chapter 4 this link of dissociative symptoms to symptom over-reporting is addressed with the same symptom, DES-item 11: "Some people have the experience of looking in a mirror and not recognizing themselves". Failure to recognize oneself in mirrors is known as the "mirror sign" (Goedhart \& Sno, 2014). Three different instruments - open interviews, an inventory of implausible symptoms, and the Dissociative Experiences Scale (DES; Bernstein 
\& Putnam, 1986) - found a similar prevalence (between 33\% and 37\%) of the mirror sign in patients at the referral center for refugee mental health mentioned above. Their average score on the DES was high ( $M=22.6, S D=21.5)$, but certainly comparable to scores that are obtained elsewhere in clinical settings (Foote et al., 2006; Friedl et al., 2000). In all subsamples of our study, endorsement of the mirror sign was found to be associated with over-endorsement of items on an inventory of implausible symptoms, a Dutch study version of the Structured Inventory of Malingered Symptomatology (SIMS; Widows \& Smith, 2005). Hall and Poirier (2011) reported a similar association in forensic samples.

Merckelbach et al. (2017) discussed various interpretations of the link between symptom over-reporting and dissociative experiences. One interpretation was the possibility of genuine symptoms overlapping with implausible symptoms. This overlap can occur if the wording of SVT items is similar to the wording of a dissociative sign, for example "Sometimes, I lose all feelings in my hand so that it is as if I have a glove on" (item 1 of the Structured Inventory of Malingered Symptomatology, SIMS, Widows \& Smith, 2005) and "I cannot feel properly the objects that I touch with my hands for it feels as if it were not me who were touching it" (item 20 of the Cambridge Depersonalization Scale, CDS, Sierra \& Berrios, 2000).

Another possibility is that the overlap originates from problematic (i.e., highly unlikely) items included in a genuine symptom inventory. This possibility is addressed in Chapter 5 and deserves to be taken seriously for several reasons. One reason concerns the neurobiological implausibility of the mirror sign. This symptom and several other dissociative symptoms imply severe cognitive impairment (e.g., in autobiographical memory), whereas neuropsychological studies have reported only subtle deficits in patients with dissociative disorders (Giesbrecht et al., 2008). But even if the failure in self-recognition during depersonalization is emotional rather than cognitive (the reflection in the mirror does not feel like one's own), the symptom remains eccentric. When patients with known depersonalization disorder are asked about the mirror sign, they report it to be "rare" or "bizarre" (Simeon et al., 1998).

But would this still be the case if these patients had a different cultural background? What is considered to be implausible may differ between cultures (Weiss \& Rosenfeld, 2012); culture may affect the way in which individuals express symptoms (e.g., Hausotter \& SchoulerOcak, 2007). Likewise, professionals from different countries may differ in their evaluation of mental symptoms (Giosan et al., 2001). To assess whether implausible symptoms (such as those listed in the SIMS) are also regarded as implausible in other cultures and regions of the world, Boskovic et al. (2017) asked professionals working in Western countries $(n=56)$ and 
professionals working in non-Western countries $(n=37)$ to rate the plausibility of uncommon symptoms taken from the SIMS, dissociative symptoms of the DES and standard symptoms (e.g., anxiety, depression) of the Brief Symptom Inventory (BSI-18; Derogatis, 2001). Western and non-Western experts did not differ in their plausibility ratings; the items of the BSI-18 were rated as more plausible than uncommon and dissociative symptoms, both of which were rated equally implausible. In comparison, patients at the referral center for refugee mental health (chapter 6) endorsed the mirror sign at the same rate as an item of the implausible symptom inventory that reads: "When I hear voices I feel as though my teeth are leaving my body".

Dissociative symptoms reported by refugee mental health patients should therefore not be taken for granted. Before pharmacological or behavioral treatments are initiated, the symptoms should be evaluated, preferably with SVTs. Of course, refugee mental health patients are not unique in this respect. The mirror sign is an implausible symptom that might serve as well as an item from a SRVT.

\section{Is the "mirror sign" an early psychotic symptom?}

Dissociative symptoms are not the only symptoms that may seem to be implausible. To observers who are unfamiliar with psychosis, the experiences described by psychotic patients may appear equally implausible. Chapter 5 describes how, after it was first described by a novelist, the psychiatric interpretation of the mirror sign historically varied between dissociation, fantasy, and psychosis (for an overview, see also Goedhart \& Sno, 2014). Similarly to dissociative patients, the failure in self-recognition in psychotic patients is emotional rather than cognitive. Psychotic patients see a reflection in the mirror as having striking similarity to their own face, but experience it as a twin or an impostor staring back. This is why the symptom has also been described as part of a psychotic misidentification syndrome, "Capgras syndrome of the Self" (Neppe, 1992). The initial open interview study in the referral center for refugee mental health (Chapter 4) was inspired by French psychiatric literature (e.g., Lacan, 1966), where it is described as an early sign of psychosis.

A substantial proportion of the patients in the referral center were classified as having a psychotic disorder during the study period (29\%; Chapter 7). Partly based on a patient sample from the same referral center for refugee mental health, Braakman et al. (2013) conducted a study on the clinical differences between schizophrenia and posttraumatic stress disorder with secondary psychotic features (PTSD-SP). One of the differences was that patients meeting criteria for PTSD-SP qualified substantially more often for co-morbid dissociative disorder 
(17.6\% vs. 5.9\%). Could the mirror sign be an early sign of PTSD-SP?

The open interview study described in this thesis did not find a robust and unique association between the mirror sign and psychosis (Van der Heide, 2007). We looked for other evidence in the student samples that are described in Chapter 5. Even though genuine depersonalization disorder patients regard the mirror sign as "rare" or "bizarre" (see above; Simeon et al., 1998), 8\%-10\% of the students indicated to have this experience at least 30\% of the time. This is in line with the findings of the population-based study of Ross et al. (1991) in Canada. Meanwhile, the lifetime prevalence of psychotic dysregulation is no more than 3\% (Perala et al. 2007). Consequently, although deficient self-recognition may be a genuine symptom in psychotic patients, or in patients with PTSD-SP for that matter, it is unlikely that it heralds impending psychotic disintegration in other groups.

\section{To what extent do deviant SVT scores of refugee mental health patients reflect their poor skills in the language of the host country rather than feigned symptoms?}

Using a differential prevalence study design, the incentive status of patients at the referral center for refugee mental health (Chapters 4, 6-9) was rated by independent evaluators using a pre-defined set of potential incentives according to a pre-defined algorithm. The incentive groups differed substantially in their SVT scores throughout the studies. One group - patients with a positive incentive to exaggerate or feign - over-endorsed unlikely symptoms significantly more often than the other incentive groups. This group also underperformed more often and to a larger extent than the other incentive groups. Group differences linked to other variables such as age, sex or region of origin disappeared after correction for incentives.

The language proficiency status of patients was evaluated in a similar manner. We hypothesized a close (and artefactual) link between proficiency and scores on SVTs; low proficiency would thus generate larger endorsement rates on self-report inventories of unlikely symptoms and possibly more errors on PVTs. We added a second strategy to discern language effects to the design by using an implausible symptom inventory with a high verbal mediation (an adapted study version of the SIMS), and a PVT with a low verbal mediation (a forced-choice task based on Morel's Emotional Numbing Test; MENT; Morel 1998). Thus, we expected the link to be more pronounced for the self-report inventory than for the PVT.

In all samples from the various studies (Chapters 4, 6-9), patients with poor language proficiency did not endorse unlikely symptoms more often than others, but they did underperform more often. In other words: proficiency was associated with test failure on low 
verbal mediation instruments, but not on an instrument with a high verbal mediation. These outcomes were unexpected, and not in line with other research that found poor proficiency to be associated with test failure on symptom validity instruments with high verbal mediation, not low verbal mediation (Erdodi et al., 2017).

A possible explanation for this discrepancy may be found in the association between poor language proficiency and outcomes of one of the various subscales of the implausible symptom inventory. Specifically, compared with other groups, patients with poor proficiency endorsed significantly more symptoms on the subscale for exaggeration of intellectual impairment. This suggests that the proficiency assessments by the Dutch language teachers may have been confounded by patients exaggerating or feigning intellectual impairment in the low proficiency group.

More in line with other research (Erdodi et al., 2017) is the finding in Chapter 8 that in detecting symptom exaggeration in various incentive groups, a low verbal mediation PVT score showed overall superior specificity compared to the high verbal mediation implausible symptom inventory.

In conclusion, the association with external incentives was considerable and was stronger than associations with other group identifiers, such as age, sex or region of origin. Deviant scores on SVTs in refugee mental health patients indicate external incentives, but we could not confirm a similar pattern for poor proficiency; the association we found was contrary to what was expected and possibly due to a confounding interaction with external incentives in our samples and how they were classified in terms of proficiency.

\section{In refugee mental health patients, is deviant performance on SVTs associated with escalated symptom endorsement on standard diagnostic instruments?}

Deviant performance on SVTs is likely to predict poor diagnostic validity only if it is associated with the outcomes of regular diagnostic tests. If endorsement of implausible symptoms is indeed a measure for exaggerated symptom presentation, then high endorsement rates on standard clinical self-report inventories are to be expected (Rogers, 2018). DandachiFitzGerald et al. $(2011,2016)$ previously documented the robust association between SVT failure and heightened scores on standard clinical instruments in a mixed-psychiatric sample in the Netherlands.

Chapter 6 describes studies with two standard instruments for self-reported symptoms in the referral center for refugee mental health: the Dissociative Experiences Scale (DES; Bernstein 
\& Putnam, 1986) and the Harvard Trauma Questionnaire (HTQ; Mollica et al., 1992). Again, average scores on these instruments were high but still in line with scores reported in other studies on similar groups (Carlson \& Rosser-Hogan, 1993; Kleijn et al., 1998). Both instruments correlated with the implausible symptom inventory; patients in the positive incentive group endorsed more symptoms in general, regardless whether they were genuine or implausible, than patients in other groups. These positive incentive patients also reported more, and more serious, traumatic events than other patients.

Likewise, DES scores correlated positively to the error scores on the PVT we used, a forced-choice task that is a research version of Morel's Emotional Numbing Test (MENT; Morel, 1998) which measures underperformance. In fact, the DES was not the only standard self-report inventory that correlated with underperformance; in all refugee mental health samples that were given the implausible symptom inventory, we found strong correlations with the forced-choice task, both in subsamples (Chapters 4 and 6) and in the grand analysis ( $n=203$; Chapter 9). All patients who failed the forced-choice task also over-endorsed implausible symptoms. This was unexpected, and not in line with the findings of DandachiFitzGerald et al. (2011, 2016). Performance validity tests (PVTs) are a type of SVT that tap into underperformance; this is a different detection strategy altogether, and a separate dimension in symptom validity assessment. If underperformance on a PVT correlates with over-endorsement of unlikely symptoms on a SRVT in the same sample, this means that both types of outcomes were biased by the same confounder, or alternatively that patients were relying on multiple feigning strategies at the same time. A factor that is shared by both tests is that they tap into symptomatology that is -somewhat misleadingly - attributed to the lasting effects of psychological trauma. Both the MENT and our forced-choice adaptation of this instrument are introduced to patients by emphasizing specifically that those with Posttraumatic Stress Disorder (PTSD) may have difficulty recognizing facial expressions of emotions due to their blunted affect. The implausible symptom inventory, a study version of the Structured Inventory of Malingered Symptomatology (SIMS; Widows \& Smith, 2005), does not involve such an introduction, but nevertheless patients in the referral center reported a wide variety of symptoms, including cognitive impairment and memory lapses, and usually insisted that their mental health did not deteriorate until certain critical life-events occurred.

The association between error scores on the PVTs and error scores on a regular cognitive task turned out to be more complex; in Chapter 8, based on a sample of 56 patients in the referral center for refugee mental health, the outcomes of both the forced-choice task and 
the Tombaugh's Test of Memory Malingering (TOMM, Tombaugh, 1996) were compared with the results on the Study Path Selection Test (Trajectkeuze Toets; SPST, Bureau InterCulturele Evaluatie, 1998), a structured assessment of verbal learning skills. We found a strong correlation between both PVTs (Spearmans' rho $=0.698, p<0.001$ ), but neither PVT correlated with the SPST. Also, the PVTs differentiated between incentive groups, whereas the SPST did not. We interpreted this as an indication that the "floor effect" strategy in the detection of poor effort was functional in the PVTs we used, despite linguistic and cultural differences in the sample and the sub-optimal presentation with dialogue interpreters in the referral center. We specifically selected the SPST because its cognitive load does not allow genuinely impaired patients to pass with sufficient effort, whereas PVTs are devised to do exactly that. That the floor effect was not unequivocally effective is discussed in the next section.

In our sample of refugee mental health patients, over-endorsement of implausible symptoms was thus associated with escalated reports of dissociative symptoms, symptoms of psychological trauma, and reports of traumatic events. This was not the case with underperformance; patients without incentive to exaggerate impairment passed the PVTs, but performed as poorly as the positive incentive group when confronted with the more realistic cognitive load of the standard SPST.

\section{Does a psychotic disorder confound the outcomes of SVTs in refugee mental health patients?}

Although the incentive groups exhibited significant differences as shown by the differential prevalence design of the study, considerable numbers of patients with an incentive to deny symptoms or minimize impairment over-endorsed implausible symptoms and failed the PVT, despite its mild cognitive load. As mentioned above, our index of linguistic/cultural differences showed an association with test outcomes that was contrary to what was expected based on previous research in the field. In the next analysis we therefore focused on confounders other than over-reporting or underperformance. A likely candidate among confounders in our data base would be psychotic disorganization.

The referral center for refugee mental health that provided most of our data admitted patients with psychotic disorders relatively often because it served as a referral center for this type of pathology for other refugee mental health centers in the Netherlands. Based on the diagnostic classifications at discharge, $29 \%$ of the inpatients suffered from a psychotic disorder (schizophrenia spectrum or other psychotic disorder; DSM IV-TR; APA, 2000). In the sample with no/mixed incentives to exaggerate symptoms, $45 \%$ of the patients were classified as 
such, compared to $79 \%$ in the sample with a negative incentive. Although current psychotic symptoms may undermine the validity of neuropsychological test outcomes (SVTs included), less than $5 \%$ of the patients were excluded from participation (Chapter 7 ). There is some evidence that psychotic patients tend to over-endorse unlikely symptoms. In the mixed psychiatric sample of Dandachi-FitzGerald et al. (2011), 21\% of the patients over-endorsed on the SIMS. In their systematic review of the SIMS, van Impelen et al. (2014) noted that: "...the ability of the original SIMS cut-offs to classify honest responders with psychopathology correctly is not satisfactory: Cut scores of $>14$ and $>16$ have generally yielded low specificity rates in honest patient samples. In patients with schizophrenia, individuals with intellectual disability, and patients suffering from psychogenic non-epileptic seizures, the SIMS generates many positive results. Certain characteristics of schizophrenia, such as deficits in reality monitoring (Radaelli et al., 2013), lack of illness insight (Shad et al., 2006), and cognitive impairment (Schaefer et al., 2013; but see Stevens et al., 2014), may predispose patients to produce high SIMS scores." Peters et al. (2013) found that $28 \%$ to $30 \%$ of their samples of patients with schizophrenia over-endorsed symptoms on the SIMS. In our sample reported in Chapter 7, we found that $27 \%$ of patients with a negative incentive over-endorsed on the SIMS (at a cut-off of 16). In contrast, Schroeder and Marshall (2011) found no differences between psychotic and nonpsychotic patients on seven different SVTs.

Does a current psychotic disorder also interfere with performance on PVTs? Morel included 17 inpatients treated for schizophrenia in his preliminary validation study of the MENT (from which our forced-choice task was derived) and found a mean error score of 5.5 $(S D=3.3)$; both the MENT and our forced-choice task version rely on a cut-off at nine errors. Nevertheless, Morel warned that psychometric tests designed to assess simulated impairment may not be appropriate for patients who are actively psychotic (Morel, 2010, p. 69). Both Morel's MENT and our forced-choice task rely on visual recognition of emotional face expressions, an ability that tends to be compromised in psychotic patients (Edwards et al., 2002). In our study reported in Chapter 7, the error scores of psychotic patients with mixed incentives or a negative incentive were found to exceed those of non-psychotic patients in the same groups. The error scores of the negative incentive group also exceeded those of healthy controls but were similar to the error scores of the sample of chronic psychotic Dutch patients on the same task (failure rates of $21 \%$ in both groups).

It is therefore likely that psychotic symptomatology compromises the diagnostic accuracy of SVTs. We advise clinicians to take this in account when they decide to use SVTs to refine their diagnostic impressions. 


\section{Do regional differences in the world confound the outcomes of SVTs in refugee mental health patients?}

Chapter 9 describes another attempt to identify additional confounders to account for the extreme SVT failure rates in the referral center for refugee mental health. In that study we also explored a measure for regional differences in culture and language that could serve as an alternative for the previously used language proficiency measure. In transcultural psychology, there has been a heated debate about the meaning of world-wide differences in "national IQ" - the average score on Western-European style IQ tests per country. Research on this topic found that "national" IQ differs around the globe, ranging from 108 in Singapore and Hong Kong to 60-70 in regions of Sub-Saharan Africa (Lynn \& Meissenberg, 2010). The cause of this disparity - whether it is genetic or an environmental artefact - has been a major source of controversy (Lynn \& Vanhanen, 2002; but also see Barnett \& Williams, 2004; Rinderman, 2007; Wicherts et al., 2010). The notion of a possible genetic factor fueled racist agendas and eugenics movements around the globe (Cafasso, 2020).

In theory, global deviancies of up to 40 from the average IQ level (100) could cause false positives on a range of cognitive tests among refugee mental health patients from such countries. To find out whether such an artefact might have affected PVT outcomes in the referral center, we compared the outcomes of the forced-choice task of patients from different regions in the world. To control for the biasing effects of external incentives, we only selected patients from the negative or mixed incentive groups for this comparison. As a differential prevalence design is a rather crude manner to define incentive status (see below), we checked these subsamples for the presence of specific learning effects that would differentiate patients with sufficient effort from those with suboptimal effort. The first effect was the learning effect during the test. If essentially the same task is presented throughout the test, patients showing sufficient effort are expected to make fewer errors towards the end of the test. With suboptimal effort, this effect is not likely to occur. The second was the learning effect between tests, in cases where same test is presented twice. Patients showing sufficient effort are likely to make a similar, or smaller, number of errors the second time. Patients with suboptimal effort may even make more errors the second time - a reverse learning effect. To study the latter effect, we presented the forced-choice task twice to patients who initially passed the test (i.e., had error scores below the cut-off); this was done the first time at admission and the second time towards their discharge. The learning effect during the test was found to be limited to the negative/mixed incentives group, whereas the reverse learning effect in between tests 
occurred only in the positive incentive group. We regard this as a confirmation of the accuracy of the procedure we used to assign patients to incentive groups.

There were no significant differences in error scores between negative/mixed incentive subsamples from different regions in the world. For the sake of completeness, we repeated the same procedure for the implausible symptom inventory and found a similar absence of a regional effect on scores. We thus found little support for the idea that differences in cultural and national background profoundly affect SVTs. However, the controversy about regional differences in average cognitive achievement is a painful reminder that African citizens still face the burden of stigma. This may even involve self-stigma, given the tendency of some well-educated, middle-class African youngsters to present themselves as illiterate street kids during their asylum procedure (Van Wijk, 2007). Hopefully, our findings will help our society to eliminate this stigma.

\section{Methodological considerations}

\section{Strengths}

To start with, it should be noted that when a proportion of up to $60 \%$ of patients in a treatment center are found to fail on two or more SVTs, this is a disastrous outcome for the staff members concerned. As a consequence, the staff of the referral center who conducted or participated otherwise in this project were highly motivated to disprove their initial findings (e.g., regular SVT failures). Even if not intentional, this is in line with a credible scientific attitude, especially the idea that one should strive for falsification.

The project in the referral center that generated most of the data for the research reported in this thesis started as quality control for the implementation of routine outcome monitoring (ROM). In itself, such monitoring is a close approximation of clinical practice in these settings. At the same time, the scientific standards were high for a routine implementation project; staff members conducting interviews were trained to do so based a protocol that included both intra- and interrater comparability checks. The mode of interviewing was standardized, interviews were done in a counter-balanced order, and regular tests and SVTS were administered independently by different staff members (Van der Heide, 2012). Assignment to incentive groups was done by experts in procedures relevant to the target group, and assignment to proficiency groups was done by experts in language training, independently and 
in a structured and predefined manner. To avoid the use of possibly confounded self-reported data, criteria for incentives were based solely on objective data in patient files about current procedures (Chapters 4, 6-9). Only clinical instruments with established validity were used. Self-report instruments like the DES and the HTQ (Chapter 4) have been validated in various refugee communities in the world; to a more limited extent, this was also the case for the SPST (Chapter 8).

Regarding the SVTS, Correa (2018) noted that the Dutch version of the SIMS (on which our implausible symptom inventory was based) meets several criteria for transcultural use: it was created in a step-wise process with translation and back-translation, and cultural specific references were replaced by Dutch equivalents. Our study version was created by replacing Dutch equivalents with culturally neutral equivalents. The Dutch version was found to have good test-retest reliability and internal consistency, with sensitivity, specificity, and positive predictive power all exceeding 0.90 (see the review by Van Impelen et al., 2014; for cultural generalizability, see Boskovic et al., 2017). Our forced-choice task was also separately validated, both in student samples and in veterans (Geraerts et al., 2009). In their review Nijdam-Jones et al. (2017) found a sensitivity of 0.89 and a specificity of 0.91 for the SIMS (see also Van Impelen et al., 2014, who are critical about the specificity of the SIMS); for the MENT, NijdamJones et al. reported a sensitivity of 0.79 and a specificity of 0.95 ; and for the TOMM, they reported accuracy statistics of 0.60 and 0.91 . One the one hand, the superior sensitivity of the SIMS could be related to our finding of larger failure rates for our implausible symptom inventory in comparison with those for the PVTs that we used. On the other hand, the PVTs discriminated better than the self-report inventory between incentive groups, which could be attributed to the higher specificity of PVTs.

The patients in the referral center for refugee mental health were tested with two and sometimes three SVTs, always with one SRVT in combination with at least one PVT. This enabled us to assess the effect of high verbal mediation in comparison with low verbal mediation. We also relied on multiple detection strategies to detect symptom distortion (i.e., self-report versus performance), thus increasing the sensitivity to detect feigning. Larrabee (2008) demonstrated this superior effect of combined SVT measures by chaining likelihood ratios of the separate tests. However, Berthelson et al. (2013) contended that the false positive rate may increase when multiple tests are used. By considering both negative and positive trials in their recalculation, Bender and Frederick (2018) found a smaller superiority effect of combined SVTs than Larrabee (Larrabee: post-test probability of feigning 0.936 to 0.973 after two trials; 0.989 
to 0.995 after three trials; Bender and Frederick: 0.569 to 0.865 after two trials, and 0.927 to 0.988 after three trials). Nevertheless, the practice of multiple tests, preferably tapping into different domains of feigning (e.g. SRVTs combined with PVTs), is recommended in the revised Slick criteria (Sherman et al., 2020).

\section{Limitations}

The differential prevalence design was best suited for the purposes of our studies, (Rogers, 2018). However, this design has important limitations. First, and germane to the design itself, it establishes no more than a correlation between test outcomes and presumed incentives. It does not identify feigning or determine the exact prevalence of feigning in the study samples.

Second, the differentiation between groups is based on presumed incentives, which is an inherently crude method. Even objectifiable incentives are typically mediated by the personal judgement of the patients and colored by their previous experiences, such as experience with care providers. Care may have been denied to them in the past, or care providers may have applied iatrogenic medical labels to the symptoms they presented (Niesten et al., 2020). Theoretically, differentiation could be refined by interviewing participants about their incentives, but in a study addressing the validity of self-report that would have been a methodological error.

The particularly strong association we found between incentives and test outcomes in relatively small samples may to some extent be an artifact of both the design of the study and the type of setting in which it was conducted: a highly specialized referral center for patients with severe psychiatric pathology, including psychotic disorders. For example, a proportion of this group was admitted compulsorily (Chapter 7). These patients would presumably have a strong incentive to deny or minimize symptoms, whereas the study focused on exaggeration or feigning of symptoms. The generalizability of our findings is therefore limited, even within the scope of refugee mental health.

We did not find an association between test outcomes and regional, cultural, and linguistic differences in the patient samples of the referral center. This is not consistent with the findings of other studies in this field (for an overview, see Correa, 2018; Nijdam-Jones et al., 2017). As mentioned above, our index of language proficiency may have been too crude. On the other hand, the comparison between global regions did not yield significant differences, even after controlling for external incentives. Consequently, the impact of regional, cultural, and linguistic factors may have been smaller in our samples than we anticipated. 


\section{Clinical considerations}

Discussions concerning the clinical implications of the study findings for the referral center often revolved around the question whether patients with one or more failures on SVTs were feigning or not. But even a feigning patient may have a genuine psychiatric disorder (see also Berry \& Nelson, 2010) or may develop one during a protracted period of psychiatric admission and treatment. Therefore this debate is not relevant for practical treatment issues. The essential implication of failed SVTs is that the psychiatric diagnosis becomes uncertain; poor symptom validity ultimately precludes diagnostic certainty. The relevant question should therefore be: How to proceed in case of doubt? Medical ethics are quite clear on this topic: "First, do no harm" and "If in doubt, abstain". If the diagnosis is uncertain or doubtful, the clinician needs to reconsider treatment, especially any potentially harmful effects. Based on these considerations, clinical practice in the referral center changed in the following ways:

1. Research. A clinical intervention study was terminated prematurely because the doubts regarding diagnostic validity precluded further inclusion of participants.

2. Medication. Patients who failed two or more SVTs were advised to switch from their current medication to an alternative treatment regimen with vitamin supplements. Even though they were duly informed that vitamin supplements are not psychopharmacological agents, almost all patients complied. Most patients were accustomed to being prescribed vitamin supplements in their country of origin and some even suspected that it was because of their marginal position as immigrants that their Dutch doctors failed to do so. To assess the effect of this intervention, we relied on our general impression and the number of reported incidents in the wards. Nevertheless, we did not observe any change in the symptoms of the patients concerned, and the number of reported incidents did not increase. See also Table 1.

3. Psychotherapy. Therapists and other staff previously focused on traumarelated symptoms and their management. For instance, if patients reported nightmares at night, they were encouraged to report on past traumatic events as well. This aspect of clinical practice changed as follows. Therapy during the day became more supportive, and patients with nightmares were encouraged to do a relaxation exercise and return to their bed. At the start of the study period, 
patients regularly displayed panic attacks or aggressive behavior during the night, sometimes followed by a transfer to a seclusion room. At the end of the study period, nocturnal agitation was no longer observed.

4. Use of coercion. The use of seclusion rooms was abolished. Together with the nocturnal agitation, the need for seclusion rooms disappeared as well.

5. Length of admission. Patients who failed two or more SVTs were discharged as soon as their medication was successfully changed to vitamin supplements. This was usually done gradually, but nevertheless the average length of an admission was reduced from 6-9 months at the start of the study period to 3-6 months at the end of the study period.

The issue of whether the patients in the referral center were feigning also had implications for the information we presented to the patients and their referring doctors or to the medical practitioners of the Immigration Services (if this was requested by the legal advisors of the patients). However, none of the patients of the referral center were classified as malingering. The differential prevalence design of the study did not allow for a precise estimate of the prevalence of feigning in the referral center. In individual cases, we found additional indicators of feigning, such as performance below chance level $(p<0.05)$, absence a of learning effect in repetitive tasks with a mild cognitive load (Chapter 9), a reverse learning effect (e.g. failing a PVT after initially passing it, Chapter 9), and the absence of any therapeutic effect or non-compliance with treatment (e.g., as evidenced by blood tests). However, according to the original Slick criteria (Slick et al., 1999) only patients with a performance below chance level would qualify for classification as 'definite malingering'. All others would, at most, be classified as 'probable malingering'. As these criteria were still being revised (Young, 2014; Sherman et al. 2020), the tests were presented in a suboptimal way (with dialogue interpreters), and essential data with regard to feigning in the target group were lacking (e.g., base rates, or data on cultural validity in specific ethnic groups), we decided that none of the patients should be classified as a malingerer. Diagnostic classifications in discharge letters were left to the clinical judgment of the therapist but were accompanied by a general caveat about limited diagnostic certainty in transcultural settings and in cases that involved ongoing legal procedures. To avoid misinterpretation, the results of SVTs were not mentioned. 
Table 1. Annual costs of psychiatric medication in the referral center for refugee mental health. Antiepileptic drugs used for mental disorders (carbamazepine, valproic acid, lamotrigine and topiramate) are included.

\begin{tabular}{cc}
\hline Year & Psychiatric medication (costs in euros) \\
\hline 2007 & 17,993 \\
2008 & 14,620 \\
2009 & 6,571 \\
2010 & 5,454 \\
2011 & 3,029 \\
\hline
\end{tabular}

\section{Directions for future studies}

The assessment of symptom validity in refugee mental health is still in an initial stage of development. To proceed, at least three steps are needed.

Until now, the referral center for refugee mental health has been the only mental health facility that used SVTs as part of a diagnostic routine in refugee mental health patients. To assess the generalizability of the findings in this specific setting, SVTs need to become part of diagnostic routines in other mental health facilities for refugee mental health, including out-patient settings. In addition, the use of SVTs as part of epidemiological research among persons seeking asylum would help to assess the validity of the outcomes of this type of research in the target group.

For the initial stages of research on symptom feigning in new target groups, as in the studies reported in this thesis, differential prevalence designs are a logical choice. For future studies, other research designs are needed, such as experimental simulation, known-group comparisons or the bootstrapping of detection strategies. Preferably these studies should not be limited to cross-sectional approaches, but have longitudinal designs.

To assess the relative effects of cultural and linguistic background variables more objectively and quantifiably, measures need to be developed that preferably do not rely on self-report. Assessments and ratings by experts are also unlikely to suffice for this purpose; the language proficiency assessments by the Dutch language teachers at the referral center and the ratings by interviewers and nurses may have been confounded by the incentive status of the patients.

Perhaps the assessments and ratings by experts, interviewers and nurses in the referral 
center were vulnerable to bias because they focused exclusively on genuine symptoms. New methods to quantify observations should include observations of discrepant or inconsistent behavior as well.

\section{Concluding remark}

The research reported in this thesis revealed questionable symptom validity in a nontrivial portion of refugee mental health patients. It is tempting to relate this to feigning or even malingering. Terms such as feigning and malingering have a range of negative connotations (e.g., Rogers, 2018), yet it is good to bear in mind that the overwhelming majority of people who feign symptoms do so because they are faced with harsh circumstances. Being a refugee or a person seeking asylum who is confronted with complex and lengthy legal and bureaucratic procedures in the host country certainly qualifies as harsh circumstances. 



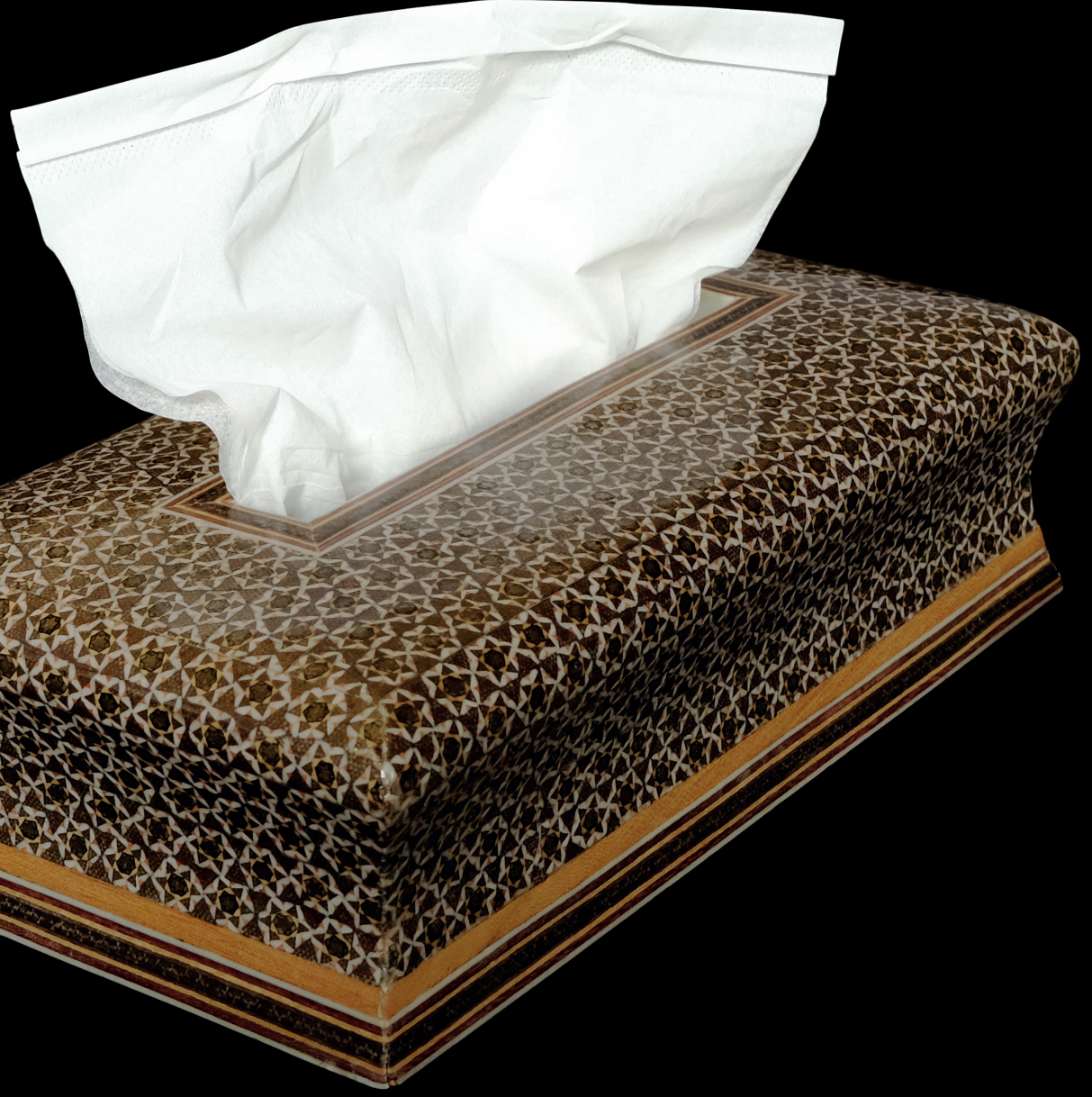


SUMMARY 
The studies in this thesis focus on the utility and clinical relevance of symptom validity assessment in refugee mental health. The few studies that have been published on this topic suggest that the usefulness of symptom validity tests (SVTs) is precarious due to the cultural and linguistic diversity of the refugee population.

Most data for this thesis were obtained at a clinical referral center for refugee mental health in the Netherlands ("Phoenix" in Wolfheze, part of ProPersona Mental Health) between 2005 and 2012. In 2005, a study into the prevalence of the mirror sign was started in this facility. In 2008, SVTs were introduced, initially as part of a project for implementing Routine Outcome Monitoring, but soon they became an integral part of the diagnostic routine of the center. Their main purpose was to establish whether standard psychological tests would be suitable for specific patients. If the results indicated that they would not, these patients were informed accordingly. Almost all patients admitted to the center participated in this program; less than $5 \%$ refused permission or were too ill to do so.

Chapter 1 provides a general introduction to the subject of symptom validity in refugee mental health. The need for its assessment to prevent unnecessary treatments and admissions is highlighted, as are the challenges posed by cultural and linguistic diversity.

Chapter $\mathbf{2}$ describes two cases of patients who were admitted to the abovementioned center. This illustrates how patients may present with severe symptoms in a consistent and credible manner throughout a protracted period of admission without their symptom overreporting being detected, but with a severe risk of iatrogenic damage.

Chapter 3 describes the case of a near fatal side-effect of medication in a refugee, who was being treated with a potentially hazardous drug (an irreversible MAO inhibitor) because of therapy-resistant symptomatology. The side-effects, which culminated in a subarachnoid hemorrhage, started shortly after the patient had used a small dose of khat.

Chapter 4 compares three samples of 85, 38, and 27 patients. We assessed how often they reported episodes with severe dissociative symptoms. Specifically, we were interested in two symptoms: not recognizing oneself in a mirror, and the experience of observing traumatic events in a mirror. We used three types of measurements. In the first sample, we used open interviews; in the second sample, an inventory of implausible symptoms was administered to which the dissociative key symptoms had been added; and in the third sample, the Dissociative Experiences Scale (DES) was given. Next, we investigated whether the outcomes, particularly reports of the key symptoms, were associated with the results of SVTs. Two SVTs were used. 
The first SVT was a Self-Report Validity Test (SRVT), which is an inventory of implausible symptoms, to screen for exaggeration of symptoms. It is based on the notion that patients who exaggerate symptoms will also be inclined to endorse unlikely symptoms. The second SVT was a Performance Validity Test (PVT). This type of test is designed in such a way that patients with genuine impairments will still make few errors, provided they exert sufficient effort. Both types of SVT use a cut-off point above which it is reasonable to suspect poor symptom validity. The SRVT was an adapted version of the Structured Inventory of Malingered Symptomatology (SIMS); and the PVT was a forced-choice task modeled after Morels' Emotional Numbing Test (MENT). In the latter test, patients are asked to identify emotions in facial expressions as depicted on photographs. For these photographs, two actors were used, a man and a woman, who posed for ten different emotions. For each item, the patient had to choose between two options (one obviously right, the other obviously wrong), that were repeated in different combinations throughout the test to decrease the cognitive load.

We also investigated whether test results were associated with external incentives of patients to exaggerate or with poor proficiency in Dutch. This part of the study used the differential prevalence method, which was also used in the studies reported in Chapters 6 through 9. As to incentives for distorting symptoms, patients in these studies were assigned to a group with a "positive" incentive to exaggerate symptoms (e.g., to obtain a residence permit), a group with a "negative" incentive, i.e. an incentive to deny symptoms (as in the case of a compulsory admission), and an intermediate group without incentives or with mixed incentives. Incentives were identified independently by the social workers of the referral center based on the patients' records. Regarding language proficiency, the Dutch language teachers of the center assigned the patients to one of three groups: a group with a poor proficiency in Dutch, a group with a good proficiency, and an intermediate group.

The two key dissociative symptoms were reported by between $27 \%$ and $63 \%$ of the patients. These are not excessive proportions compared with other clinical centers. However, patients who reported these symptoms did have elevated SRVT and PVT scores. Symptom validity was poor: between $63 \%$ and $87 \%$ of the patients endorsed too many implausible symptoms, and between $41 \%$ and $58 \%$ made too many errors on the forced-choice task. There was a strong association of suspicious symptom validity with positive incentives, but not with poor proficiency. We concluded that symptom validity should not be taken for granted in this target group, and that both an SRVT and PVT may yield important information about this.

Chapter $\mathbf{5}$ focusses on one of the symptoms discussed of the previous chapter: the 
experience of not recognizing oneself in the mirror, which is known as the "mirror sign". According to French psychoanalyst Lacan and his followers, it heralds a psychotic breakdown; according to Anglo-Saxon authors, it is a dissociative symptom that reflects depersonalization. Using groups of students as subjects, we investigated both options. Too many students endorsed this key symptom to conceptualize it as a possible pre-psychotic phenomenon. A sizable number of students also endorsed a symptom in which self-recognition is actually supposed to be intact during depersonalization. Furthermore, endorsement of the mirror sign was found to be associated with endorsement of implausible symptoms on a SRVT. Although the mirror sign may indeed be a psychotic symptom, in subjects who are not psychotic it indicates exaggeration of symptoms more than anything else.

Chapter 6 describes a study with two samples, one with 27 patients and one with 35 patients. We related SRVT and PVT scores to the outcomes of two standard psychological tests that are frequently used in refugee populations: the DES, a standard measure of dissociative symptomatology, in the first sample, and the Harvard Trauma Questionnaire (HTQ), a self-report measure of distressing events, in the second. Again, a sizable proportion of patients endorsed too many implausible symptoms on the SRVT (63\% in the first group and $83 \%$ in the second) or made too many errors on the PVT (41\% and $71 \%$, respectively). The SRVT and PVT scores were associated with the outcomes of the standard tests. All test outcomes were associated with positive incentives, but not with poor language proficiency. These findings indicate that the validity of standard psychological tests to assess symptom severity or treatment progress in this target group should not be taken for granted.

Chapter $\mathbf{7}$ describes a study that focuses on additional confounding effects such as a current psychosis. Earlier studies confirmed confounding effects of psychotic symptoms on the outcomes of the SIMS in native Dutch patients. The forced-choice task we used in previous studies (see above) is based on the intact ability to recognize emotions in facial expressions, but this may be impaired in psychotic patients. Based on a sample of 80 patients who had no incentive to exaggerate, we compared PVT performance of those with a diagnosis of psychosis and those without. The psychotic patients made significantly more errors. Next, we selected 24 patients with a negative incentive, of which $79 \%$ had been diagnosed with a psychotic disorder. We compared their error scores with error scores of 47 native Dutch, chronic psychotic patients and with error scores of 51 healthy controls. The proportion of refugee mental health patients who failed the PVT was similar to the proportion in the native Dutch, chronically psychotic patients (21\%), and significantly exceeded the proportion in healthy controls (2\%). 
We regard this as preliminary evidence that PVTs may be confounded by psychotic symptoms - or antipsychotic medication - in refugee mental health patients. Psychotic symptoms could also be also be partly responsible for PVT failure in the negative incentive group.

Chapter $\mathbf{8}$ focuses on the "floor effect" rationale of PVTs, which is used to detect suboptimal performance. In patients with psychotic symptoms, intellectual impairment or traits such as alexithymia, it tends to fail in up to $25 \%$ of the cases. This is because even simple tasks may put too much cognitive load on patients in these groups who do show sufficient effort. In addition, refugee mental health patients are faced with the effects of differences in language and culture.

We included 56 inpatients from the refugee mental health center in a study to determine whether the floor effect strategy is also usable in refugee mental health patients with severe psychiatric symptoms. These patients were assessed with the forced-choice task described above as well as with an additional PVT: Tombaugh's Test of Memory Malingering (TOMM). Patients also completed a standard cognitive test: the Study Path Selection Test (SPST). This test is used by Dutch language teachers to assess whether patients are suitable for fast-track or slow-track language classes. In comparison to patients with an incentive to exaggerate their impairment, patients without such an incentive made significantly fewer errors on the PVTs, but not on the SPST. The floor effect strategy thus appears to be usable in refugee mental health patients, even when they suffer from severe psychiatric morbidity.

Chapter 9 describes a study that investigated another a factor that may confound SVT outcomes: the effect of regional differences. We previously found that poor proficiency in Dutch is not associated with over-endorsement of implausible symptoms, but is associated with increased error scores on the forced-choice task. This was unexpected, as the forced-choice task has minimal verbal mediation in comparison to the implausible symptom inventory. After closer analysis, we found that one of the subscales of the implausible symptom inventory, a scale that screens for exaggeration of low intellect, did correlate with poor proficiency. We considered two possible explanations for this correlation: 1) the proficiency estimates by the Dutch language teachers were confounded by patients exaggerating intellectual impairment in the low proficiency group; 2) this type of test, which is tailored to American and European patients, is genuinely too difficult for some patients from other regions. We tested the latter hypothesis in a sample of 203 patients from Africa, the former Soviet Union, the Middle East, East Asia, and the former Yugoslavia. Next, the mean error scores on the forced-choice task of the regions were compared. After we limited the comparison to patients without incentives to 
exaggerate (either no incentive or a negative incentive), group differences between the regions were no longer significant. This analysis was also conducted for the implausible symptom inventory, with a similar result.

This chapter also describes a check on our method of assigning patients to incentive groups. This check was carried out in two ways. First, we investigated whether patients were inclined to make fewer errors towards the end of the forced-choice task, indicating a learning effect. This was only found in patients without an incentive to exaggerate. Next, we selected patients who completed the forced-choice task twice, and passed it the first time. The first time, it was presented at admission and the second time before discharge. Only patients without incentives had comparable error scores at admission and discharge measurements; patients with an incentive to exaggerate were inclined to make considerably more errors before discharge. Our conclusions were as follows: 1) Our assessment of incentive status and the corresponding assignment to incentive groups is useful; 2) Although regional differences in culture and education may indeed explain differences in standard test results, the results of this study do not support the notion that these differences also affect PVT or SRVT outcomes.

Chapter 10 consists of a general discussion of the limitations and strengths of the studies included in this thesis, their implications for clinical practice, and avenues for future research.

Four limitations should be noted: 1) A differential prevalence design allows for inferences about association of test outcomes with incentives, but not about the prevalence of actual malingering in a sample; 2). In our design, we did not include "internal" incentives, which patients may not be aware of, but that can still affect test outcomes; 3 ). Due to the lack of validated translations, professional interpreters assisted in the presentation of the test materials. Although this method does reflect clinical practice, it is also prone to error; 4) Only clinical refugee mental health patients in a highly specialized facility were included in the studies presented in this thesis, so the outcomes cannot be generalized to other refugee mental health settings, such as outpatient departments.

Several strengths of our studies should also be noted. First, almost all patients who were admitted to the refugee mental health center participated in the studies, and the testing was done in a manner that reflects clinical practice in this type of center. Although the findings in our studies cannot be generalized to outpatient settings, they are still highly informative for clinical practice in refugee mental health. Second, even though the outcomes of our studies were potentially disadvantageous to the referral center concerned, the staff who conducted the studies in this center demonstrated scientific integrity in line with the best traditions in 
scientific research by their attempts to refute their own findings.

Regarding implications for clinical practice, the studies showed that SVTs are useful and relevant to the target group, that they may be used to avoid unnecessary treatments and admissions, and that they can reduce the amount of coercion that may accompany admission. Future research into symptom validity assessment should be expanded to other areas in refugee mental health, such as outpatient settings or medical facilities in refugee camps. When knowledge and experience in the field accumulates in this way, other designs that enable base-rate estimates of malingering will become possible. The current cross-sectional approach may be improved by longitudinal study designs. Furthermore, there is a need for new, reliable measures for the impact of differences in language and culture.

\section{Concluding remark}

The research in this thesis indicates that the validity of the symptoms as presented by a sizable proportion of the patients in the refugee mental health center should not be taken for granted. It is tempting to assume that this subgroup feigned or malingered symptoms. However, it is important to note that in general, patients do not feign or malinger for antisocial or criminal purposes, but rather to cope with harsh, or even inhuman circumstances. Unfortunately, persons seeking asylum and refugees are still faced with such circumstances. 


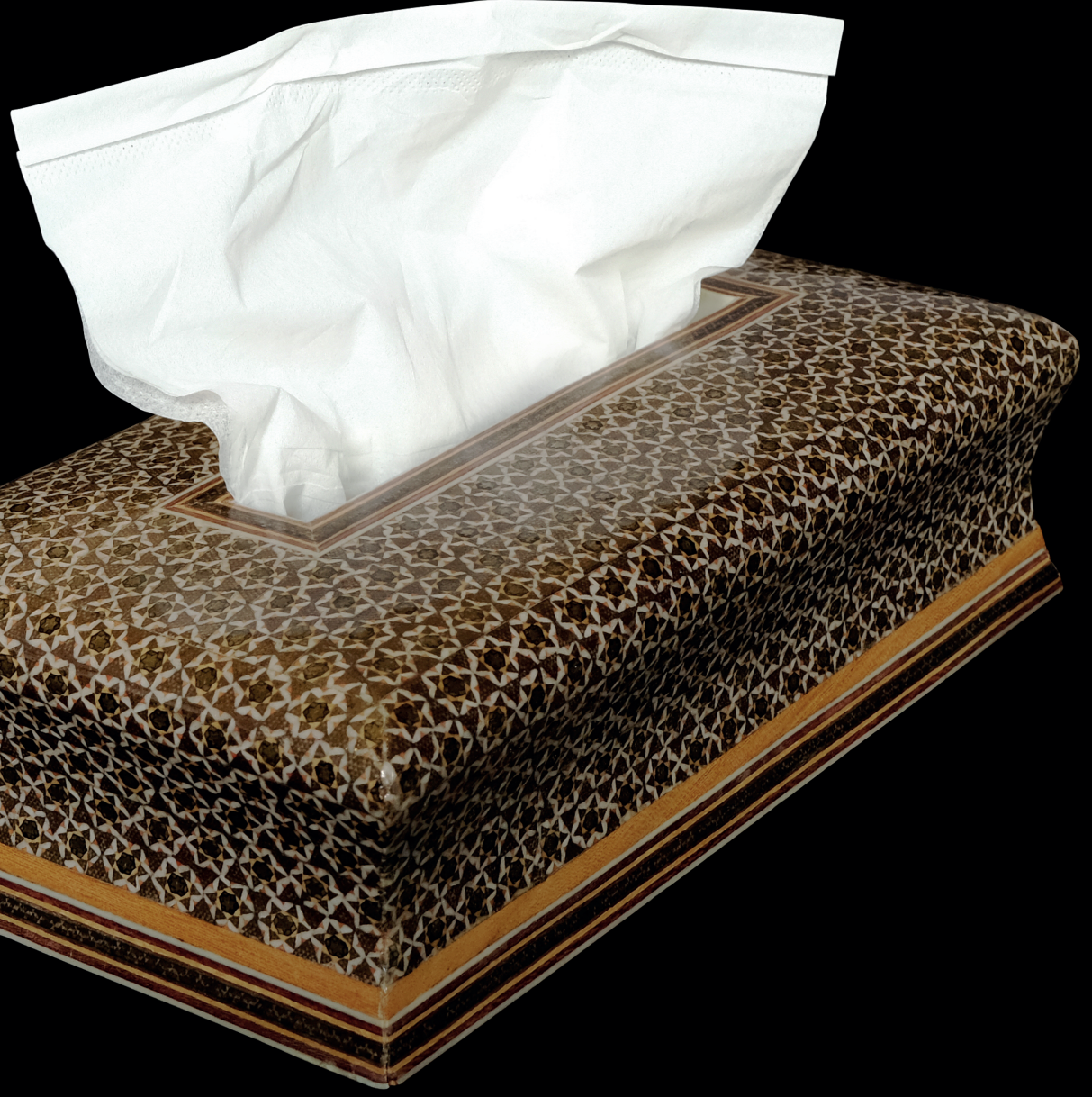


SAMENVATTING 
Het onderzoek in dit proefschrift is gericht op de bruikbaarheid en de klinische relevantie van tests voor symptoomvaliditeit in de geestelijke gezondheidzorg voor asielzoekers en vluchtelingen ("vluchtelingen-ggz"). Dit soort tests (zgn. symptoomvaliditeitstests, of SVTs) wordt nauwelijks gebruikt bij deze doelgroep. De weinige studies die er zijn over dit onderwerp, suggereren dat SVTs beperkt bruikbaar zijn omdat testuitkomsten worden vertekend door taal- en cultuurverschillen.

De data voor dit onderzoek zijn afkomstig uit een afdeling voor vluchtelingen-ggz (de afdeling "Phoenix" van ProPersona in Wolfheze). In 2005 werd hier een onderzoek gestart naar de prevalentie van het zgn. "spiegelteken", een psychiatrisch symptoom. In 2008 werden SVTs voor het eerst gebruikt in deze afdeling; aanvankelijk nog als kwaliteitsindicators bij het implementeren van psychologische vragenlijsten voor Routine Outcome Monitoring, maar na verloop van tijd werden ze onderdeel van de diagnostische routine in de kliniek. Ze waren in de eerste plaats bedoeld om te kijken of psychologische testdiagnostiek bruikbaar zou zijn bij een patiënt; bleek dat niet het geval, dan werd deze daarover ingelicht. Bijna alle opgenomen patiënten werden getest; minder dan $5 \%$ weigerde toestemming of was te verward om die te geven.

Hoofdstuk 1 biedt een algemene inleiding in het onderwerp van symptoomvaliditeit in de vluchtelingen-ggz. Het benadrukt de noodzaak om de validiteit van symptomen te bepalen in deze vorm van ggz om schade door onnodige behandeling en opname te voorkomen, maar bespreekt ook de moeilijkheden die dat met zich meebrengt vanwege verschillen in taal en cultuur.

Hoofdstuk 2 beschrijft twee gevallen van patiënten die waren opgenomen in de bovengenoemde afdeling voor vluchtelingen-ggz en laat zien hoe deze patiënten erin slaagden om op een consistente en geloofwaardige manier ernstige symptomen te veinzen tijdens een langdurige opname, zonder ontdekt te worden - maar met een fors risico op iatrogene schade.

Hoofdstuk 3 beschrijft het geval van een bijna dodelijke bijwerking van medicatie bij een vluchteling, die was ingesteld op een risicovol type medicatie (een irreversibele MAO-remmer), omdat zijn symptomen niet hadden gereageerd op meer gebruikelijke behandelingen. De bijwerking, een hersenbloeding, trad op vlak nadat hij een kleine hoeveelheid khat had gebruikt.

Hoofdstuk 4 In dit hoofdstuk worden drie steekproeven van respectievelijk 85, 38 en 27 patiënten vergeleken, waarbij telkens werd onderzocht hoe vaak ze episodes met ernstige dissociatieve symptomen rapporteerden. Het ging om twee specifieke symptomen; een waarbij 
de patiënt zegt zichzelf niet meer in de spiegel te herkennen; en een waarbij de patiënt zegt beelden van traumatische gebeurtenissen in de spiegel te zien. De symptomen werden op drie verschillende manieren uitgevraagd. In de eerste steekproef met een open interview; in de tweede steekproef via een vragenlijst met onwaarschijnlijke symptomen, waaraan deze twee symptomen waren toegevoegd; en in de derde steekproef werd een vragenlijst voor dissociatieve symptomen afgenomen, namelijk de veel gebruikte Dissociative Experiences Scale (DES).

Vervolgens werd bepaald of de uitkomsten geassocieerd waren met afwijkende uitslagen op een SVT. Hiervoor werden twee SVTs gebruikt. De eerste was een zgn. Self Report Validity Test (SRVT); het ging om een lijst met onwaarschijnlijke symptomen die gebaseerd is op de aanname dat mensen die symptomen willen overdrijven de neiging hebben om onwaarschijnlijke symptomen aan te vinken. De tweede was een test die kijkt of de patiënt zich voldoende heeft ingespannen voor een goed testresultaat, een zgn. Performance Validity Test (PVT). Die zijn zo gemaakt dat patiënten met echte beperkingen toch weinig fouten maken zo lang ze voldoende hun best doen. Beide soorten tests hanteren een afkappunt: boven een bepaald aantal aangekruiste symptomen of fouten wordt gesproken van onvoldoende validiteit. Als SRVT werd een aangepaste versie van de Structured Inventory of Malingered Symptomatology (SIMS) gebruikt; en als PVT een tweekeuze taak naar voorbeeld van de Emotional Numbing Test van Morel (MENT). Dat is een test waarbij de patiënt moet proberen een bepaalde emotie te herkennen aan de hand van een gezichtsuitdrukking op een foto. Voor de foto's zijn twee acteurs gebruikt, een man en een vrouw, die tien verschillende emoties uitbeelden. De patiënt moet telkens kiezen uit twee antwoordopties (waarvan een goed en een fout), die in wisselende samenstellingen tijdens de test herhaald worden om het makkelijk te maken.

Ook werd gekeken of de testuitslagen samenhingen met een belang van patiënten bij het overdrijven van symptomen of met een slechte beheersing van de Nederlandse taal. Dat werd gedaan in een opzet die bekend staat als de differentiële prevalentie-methode. Deze methode werd ook gebruikt in hoofdstukken 6 - 9. Ze bestaat eruit dat testuitslagen worden gerelateerd aan de belangen die patiënten hebben om hun symptomen anders voor te stellen dan ze in werkelijkheid zijn. In dit proefschrift werden patiënten verdeeld in een groep met een "positief" belang om symptomen te overdrijven (bijvoorbeeld, voor het verkrijgen van een verblijfsvergunning), een groep met een "negatief" belang - een belang om symptomen te ontkennen (bijvoorbeeld bij een onvrijwillige opname) en een groep daar tussenin- zonder belangen, of met zowel een positief als een negatief belang. De aanwezigheid 
van positieve of negatieve belangen werd onafhankelijk van het testonderzoek vastgesteld door de maatschappelijk werkers van de afdeling aan de hand van de medische dossiers van de patiënten. Ondertussen verdeelden de leraren Nederlands van de afdeling, ook onafhankelijk van het testonderzoek, de patiënten in een groep die nog slecht Nederlands sprak, een groep die al goed Nederlands sprak en een groep daar tussenin.

De twee dissociatieve symptomen werden in de verschillende samples gerapporteerd door een deel van de patiënten dat varieerde tussen de $27 \%$ en 63\%. Dat zijn geen ongebruikelijke percentages voor een klinische afdeling. Patiënten die de symptomen rapporteerden hadden wel vaker (te) hoge SRVT- of PVT scores. De symptoomvaliditeit was problematisch: $63 \%$ tot $87 \%$ van de patiënten gaven teveel onwaarschijnlijke symptomen aan, en $41 \%$ tot $58 \%$ maakte teveel fouten bij de tweekeuze toets. Er werd een sterke associatie gevonden met belangen om symptomen te overdrijven, maar niet met een slechte beheersing van het Nederlands. Onze conclusie is dat de validiteit van door patiënten uit deze doelgroep gerapporteerde symptomen niet vanzelfsprekend is en dat zowel een SRVT als een PVT in dat verband belangrijke informatie kunnen opleveren.

Hoofdstuk 5 gaat nog eens in op één van de twee symptomen uit het vorige hoofdstuk: de ervaring dat je jezelf niet herkent in de spiegel, ook wel bekend als het "spiegelteken". Volgens de Franse psychoanalyticus Lacan en zijn volgelingen is het een aanwijzing voor een op handen zijnde psychotische ontregeling. Engelstalige auteurs zien het meer zien als een teken van depersonalisatie, een dissociatief symptoom dus. We onderzochten beide opties aan de hand van steekproeven onder studenten. Er waren teveel studenten die het spiegelteken rapporteerden om er redelijkerwijze een aanwijzing voor een op handen zijnde psychose in te kunnen zien. Een niet onaanzienlijk aantal studenten kruiste bovendien een symptoom aan dat juist wèl veronderstelt dat je jezelf herkent in de spiegel tijdens depersonalisatie. Verder bleek dat studenten die het spiegelteken rapporteerden ook vaker onwaarschijnlijke symptomen omarmden op een SRVT. Onze conclusie: het is best mogelijk dat het spiegelteken voorkomt bij psychotische patiënten, maar in andere groepen wijst het waarschijnlijk eerder op het overdrijven van symptomen dan op iets anders.

Hoofdstuk 6 beschrijft een studie met twee steekproeven van 27 en 35 patiënten. Dezelfde SRVT en PVT als in hoofdstuk 4 werden gebruikt, en de uitkomsten daarvan werden gekoppeld aan die van twee courante klinische vragenlijsten. In de eerste steekproef was dat de al eerder genoemde DES en in tweede steekproef de Harvard Trauma Questionnaire (HTQ). Beide instrumenten worden regelmatig ingezet in de vluchtelingen-ggz. Opnieuw omarmde 
een aanzienlijk deel van de patiënten teveel onwaarschijnlijke symptomen (respectievelijk 63 en $83 \%$ ) en/of maakte teveel fouten bij de tweekeuze-toets (respectievelijk 41\% en 71\%). De uitkomsten van beide SVTs bleken geassocieerd met de uitkomsten van de courante tests: bij een overmaat aan onwaarschijnlijke symptomen of fouten vielen de scores op de DES en de HTQ eveneens hoog uit. Ook bleken alle testresultaten sterk geassocieerd met belangen om te overdrijven, maar niet met een slechte beheersing van het Nederlands. Onze conclusie is dat deze bevindingen aanleiding geven om te twijfelen aan de validiteit van courante klinische schalen als het gaat om het meten van de ernst van symptomen of de voorgang van de behandeling in deze doelgroep.

Hoofdstuk 7 beschrijft een studie die zich richt op de mogelijke vertekenende effecten van psychotische symptomen op de uitkomsten van PVTs. Eerdere studies vonden zulke effecten al wel voor de SIMS bij Nederlandse psychiatrische patiënten. Bovendien is het vermogen om gezichtsuitdrukkingen accuraat op emotionele waarde te taxeren nogal eens verminderd bij psychotische patiënten. In een groep van 80 patiënten die geen belang hadden bij overdrijven van hun symptomen werd het aantal fouten op de tweekeuze-toets van patiënten met een psychotische aandoening vergeleken met dat van patiënten zonder een dergelijke diagnose. De psychotische patiënten maakten significant meer fouten. Vervolgens verzamelden we 24 patiënten die belang hadden bij het ontkennen van hun symptomen; $79 \%$ van deze groep had als diagnose een psychotische stoornis. We vergeleken het aantal fouten dat zij maakten met dat van een groep van 47 Nederlandse patiënten met een chronische psychotische aandoening en met 51 gezonde controles. De patiënten van de afdeling voor vluchtelingen-ggz maakten even vaak teveel fouten als de Nederlandse psychotische patiënten (21\%), maar dat percentage lag wel substantieel boven de twee procent gezonde controles die teveel fouten maakten. We beschouwen dit als een voorlopig bewijs dat psychotische symptomen - of mogelijk de medicatie die daartegen gebruikt wordt- de uitkomsten van PVTs vertekenen in deze doelgroep. We beschouwen dat ook als een van de mogelijke verklaringen voor het deel van de patiënten dat ondanks een "negatief" belang toch teveel fouten maakte op de tweekeuze-toets.

In Hoofdstuk 8 wordt de strategie die bij een PVT gebruikt wordt om te bepalen of een patiënt voldoende inspanning vertoont voor een optimaal testresultaat - "floor effect"- nader onderzocht. Die strategie faalt bij patiënten met psychotische symptomen, zwakbegaafdheid of bijvoorbeeld alexithymie tot in $25 \%$ van de gevallen omdat een ogenschijnlijk simpele test toch te zwaar blijkt voor patiënten met echte beperkingen die wel hun best doen. Patiënten in de vluchtelingen-ggz worden bovendien gehinderd door de verschillen in taal en cultuur. 
Om te kijken of deze strategie nog werkt bij patiënten in de vluchtelingen-ggz met ernstige psychiatrische symptomen werden 56 patiënten getest met de tweekeuze-toets en nog een andere PVT, namelijk Tombaugh's Test of Memory Malingering (TOMM). Daarnaast vulden de patiënten de trajectkeuze-toets in, een toets die werd afgenomen door de leraren Nederlands om te bepalen of een patiënt in aanmerking kwam voor een snel leertraject of juist een lang traject nodig had. Patiënten zonder belang bij het overdrijven van symptomen (geen belang of een negatief belang) maakten significant minder fouten op beide PVTs dan patiënten die daar wel een belang bij hadden, maar bij de trajectkeuze toets was dat niet het geval. Onze conclusie is dat de PVT strategie kennelijk toch werkt bij patiënten in de vluchtelingen-ggz met symptomen die ernstig genoeg zijn om ervoor te worden opgenomen.

Hoofdstuk 9 beschrijft een studie die opnieuw kijkt naar factoren die de uitkomsten van SVTs kunnen vertekenen. In dit geval de verschillen die kunnen optreden in testuitkomsten als gevolg van regionale verschillen in de wereld. Tot dusverre bleek een slechte beheersing van het Nederlands alleen geassocieerd met hoge scores op de tweekeuze-toets en niet met het op grote schaal aanvinken van onwaarschijnlijke symptomen. Dat was niet verwacht omdat de tweekeuze-toets veel minder talig is dan de vragenlijst met onwaarschijnlijke symptomen. Bij nadere analyse bleek een subschaal van de vragenlijst met onwaarschijnlijke symptomen, een schaal die screent voor het veinzen van een lage intelligentie, wel geassocieerd met slechte beheersing van het Nederlands. Mogelijk zijn er dus twee verklaringen te geven voor de gevonden discrepantie. Ofwel, patiënten die volgens de leraren Nederlands de taal slecht beheersten hadden zich dommer voorgedaan dan ze waren, of dit soort tests, die immers ontwikkeld zijn voor gebruik bij Amerikaanse of Europese patiënten, zijn werkelijk te moeilijk voor sommige patiënten uit andere delen van de wereld. Om deze laatste hypothese te toetsen werden 203 patiënten verdeeld in regio's van herkomst: Afrika, de voormalige SovjetUnie, het Midden Oosten, Oost Azië en voormalig Joegoslavië. Vervolgens werd het aantal fouten dat ze maakten op de tweekeuze-toets per regio vergeleken. Zodra we ons daarbij beperkten tot patiënten zonder belang bij het overdrijven van hun symptomen vonden we geen significante verschillen meer. Dit was ook het geval toen we de gemiddelde scores op de lijst met onwaarschijnlijke symptomen per regio vergeleken.

Verder wordt in dit hoofdstuk ook nog een vergelijking beschreven die werd uitgevoerd om onze groepsindeling voor de differentiële prevalentie-methode nog eens extra te controleren. Dat werd op twee manieren gedaan. Eerst onderzochten we of patiënten in de loop van de tweekeuze-toets minder fouten gingen maken vanwege het leereffect. Dat bleek 
alleen het geval bij patiënten die geen belang hadden bij het overdrijven van hun beperkingen. Vervolgens keken we naar een groep patiënten bij wie de tweekeuze-toets twee keer was afgenomen en die de eerste keer weinig fouten hadden gemaakt. De eerste afname was aan het begin van hun opname, de tweede tegen het einde ervan. Alleen patiënten zonder belang bij het overdrijven van hun beperkingen hadden beide keren vergelijkbare scores; patiënten met een belang om te overdrijven maakten de tweede keer aanzienlijk meer fouten.

Onze conclusie is dat onze groepsindeling inderdaad een onderscheid lijkt te maken tussen verschillende belangen. En verder, dat er ongetwijfeld verschillen zijn in testuitkomsten die aan taal- en cultuurverschillen geweten kunnen worden, maar dat de uitkomsten van het onderzoek in dit hoofdstuk niet bevestigen dat zulke verschillen ook maatgevend de uitkomsten van een PVT of een SRVT beïnvloeden.

Hoofdstuk 10 is de plek voor een algemene discussie van de studies die in dit proefschrift worden beschreven; zwakke punten, sterke punten, implicaties voor de kliniek en aanwijzingen voor toekomstig onderzoek.

Beperkingen zijn onder andere: 1. De differentiële prevalentie-methode dient om een associatie te onderzoeken tussen testuitslagen en mogelijke belangen. Ze kan niet gebruikt worden om aan te geven welk deel van de patiënten daadwerkelijk heeft gesimuleerd. 2. In deze opzet worden "interne" belangen niet meegenomen. Daarmee wordt gedoeld op impliciete belangen waarvan patiënten zich mogelijk zelfs niet bewust zijn - bijvoorbeeld, eerdere ervaringen waarbij hulp werd geweigerd. Dit soort ervaringen kan ook leiden tot een overdreven presentatie van symptomen. 3. Door het gebrek aan gevalideerde vertalingen van testen moest in de kliniek gebruik gemaakt worden van professionele tolken bij het aanbieden van de testen. Dat sluit op zich goed aan bij de klinische praktijk, maar deze manier van testafname geeft mogelijk ook meer vertekening van de testuitkomsten. 4. In dit proefschrift zijn alleen patiënten onderzocht die waren opgenomen in een gespecialiseerde kliniek. Hun testuitkomsten zeggen niet veel over andere groepen, bijvoorbeeld in de ambulante vluchtelingen-ggz.

Sterke punten zijn onder andere dat bijna alle patiënten meededen aan het onderzoek en dat de manier waarop de tests werden afgenomen een goede afspiegeling is van de klinische praktijk. De uitkomsten zeggen dan misschien weinig over de vluchtelingen-ggz in het algemeen, maar geven wel een goede indruk van het soort afdeling dat werd onderzocht. Verder is het goed om te bedenken dat de uitkomsten van dit onderzoek zeer ongunstig waren voor de afdeling waar het werd uitgevoerd. De medewerkers van de afdeling die hielpen bij de uitvoering van het onderzoek waren, geheel in de geest van de beste onderzoekstradities, zeer gemotiveerd 
om hun eigen bevindingen te weerleggen.

Voor wat het klinisch handelen aangaat, betekenen de resultaten van deze studies dat SVTs ook in de vluchtelingen-ggz gebruikt kunnen worden om behandelingen en opnames die niet nodig zijn te voorkomen en om te helpen dwang en drang terug te dringen als een opname toch nodig is. Voor toekomstig onderzoek is het van belang dat SVTs ook in andere delen van de vluchtelingen-ggz uitgeprobeerd gaan worden, bijvoorbeeld in ggz-poliklinieken of in de medische voorzieningen van vluchtelingenkampen. Als er meer ervaring komt met het testen van symptoom validiteit in de vluchtelingen-ggz, wordt het ook mogelijk om andere vormen van studieopzet te gebruiken waarmee wel iets te zeggen valt over de prevalentie van simulatie. Sowieso zou het een vooruitgang zijn als het huidige cross-sectionele onderzoek werd uitgebreid met longitudinale studies. Daarnaast is er nog steeds veel behoefte aan het ontwikkelen van goede, objectiveerbare maten voor het meten van de invloed van taal en cultuur op testuitkomsten.

\section{Slotopmerking}

De studies in dit proefschrift laten zien dat de validiteit van de symptomen die door een aanzienlijk deel van de patiënten in deze kliniek voor vluchtelingen-ggz werd gerapporteerd ver onder de maat was. Het ligt dan voor de hand om te denken aan veinzen of simuleren. In dat verband is het goed om te bedenken dat mensen meestal niet veinzen vanuit antisociale of criminele overwegingen, maar om harde, of zelfs onmenselijke omstandigheden het hoofd te bieden. Helaas worden asielzoekers en vluchtelingen nog steeds geconfronteerd met dergelijke omstandigheden. 



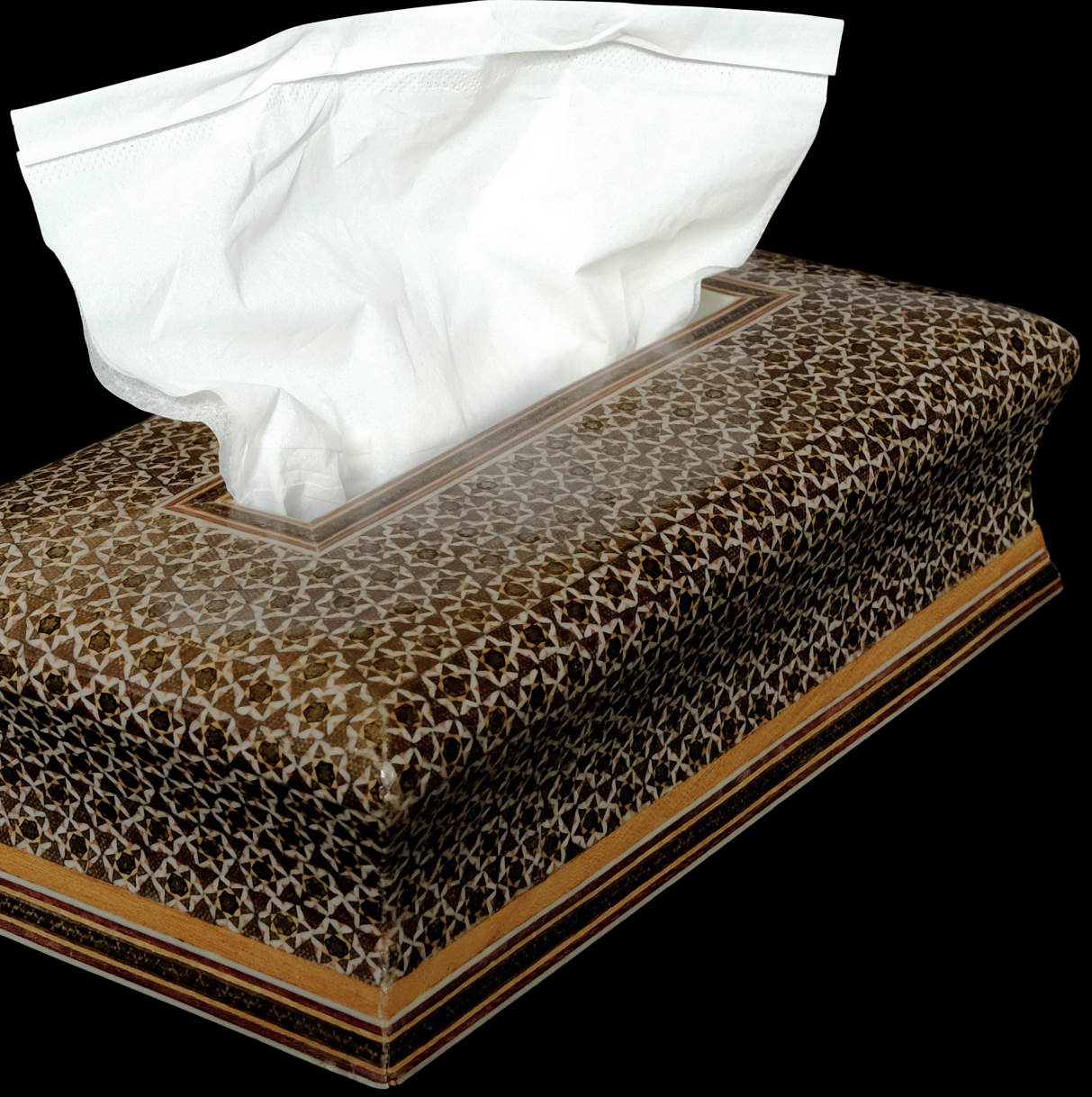


IMPACT 
Events in recent decades have shown that refugees - regardless of whether they are attempting to escape political, religious or ethnic persecution, the atrocities of war or economic disparity - are willing to endure extreme hardship. Indeed, some are prepared to risk their lives and that of their loved ones to reach the country of their choice. The research presented in this thesis could have a significant impact by alerting the mental health community to the possibility that some refugees are also prepared to endure psychiatric treatments and admissions for the sake of a residence permit.

Symptom validity experts have assumed that patients who distort their symptoms because of external incentives will avoid the harmful consequences of feigned illness that factitious disorder patients are willing to face (Feldman, 2004; Yates et al. 2018). However, the findings in this thesis suggest that this assumption underestimates the despair and determination of refugee mental health patients (Chapter 2). This is all the more problematic, as this steadfast preparedness to endure protracted periods of psychiatric treatment may initiate an escalating cycle that prompts psychiatrists to prescribe ever more hazardous types of medication (Chapter 3 ) and patients to exaggerate their complaints even more with the aim of being granted yet another extension of treatment (Frueh et al. 2005).

At the same time, our knowledge about prevalence rates and treatment effects in refugee mental health patients is almost entirely based on research designs that monitor selfreported symptoms without a check on symptom validity. An impressive body of epidemiological research has accumulated in this way, repeatedly confirming staggering rates of psychiatric morbidity among refugees (Blackmore et al. 2020) - rates that even tended to increase during asylum procedures (Laban, 2004). Nevertheless, experts in refugee mental health continue to assert that intentional over-reporting of symptoms is rare among their patients, and that it is easily detected by experienced practitioners (se.g., Houtekamer, 2012). In both clinical practice and research, diagnostic classifications are made without checking symptom validity, even though these patients may be involved in legal procedures that are crucial to their residency status (se.g., Braakman et al., 2013).

My discussions on these issues with colleagues in refugee mental health ultimately revolved around two topics. The first involved the assumption that poor intercultural validity of symptom validity tests (SVTs) preclude their use in refugee mental health. The findings reported in this thesis contradict this assumption. Strategies that are effective in American and European patients - implausible symptom endorsement and the floor effect - were also found to be effective in refugee mental health patients (Chapters 6 and 8). As reported in this 
thesis, SVT outcomes in refugee mental health patients were confounded by various factors, such as psychotic symptoms, but these factors also confound outcomes in American and European patients (Chapter 7). SVTs do not suffice to establish malingering in refugee mental health patients, but this also applies to other target groups. However in refugee mental health, as in other target groups, SVTs can effectively detect insufficient symptom validity. Indeed, if the outcomes of SVTs regarding symptom validity are taken into account, this may prevent unnecessary treatments. At the referral center for refugee mental health that provided most of the data for this thesis, the use of SVTs reduced polypharmacy, the duration of admissions, and the need for coercive measures (Chapter 10).

The second topic concerned the possible political ramifications of the findings in this thesis. Many colleagues were concerned that these findings could encourage politicians to press for more restrictive immigration policies, which would also affect the prospects of genuinely vulnerable immigrants. This ethical dilemma touches upon the independence and objectivity of scientific reporting. Although withholding or even censoring this reporting might benefit vulnerable individuals in the short term, it would ultimately undermine the credibility of science. In a society that allows its citizens to exercise freedom of belief and expression independently of political, religious or ethnic authority, credible science is a crucial asset. These citizens need access to credible facts, and science can provide such facts only if it remains independent and objective. The findings in this thesis, after being peer reviewed and published, could have additional impact by demonstrating this independence and objectivity even in the face of an ethical dilemma. 


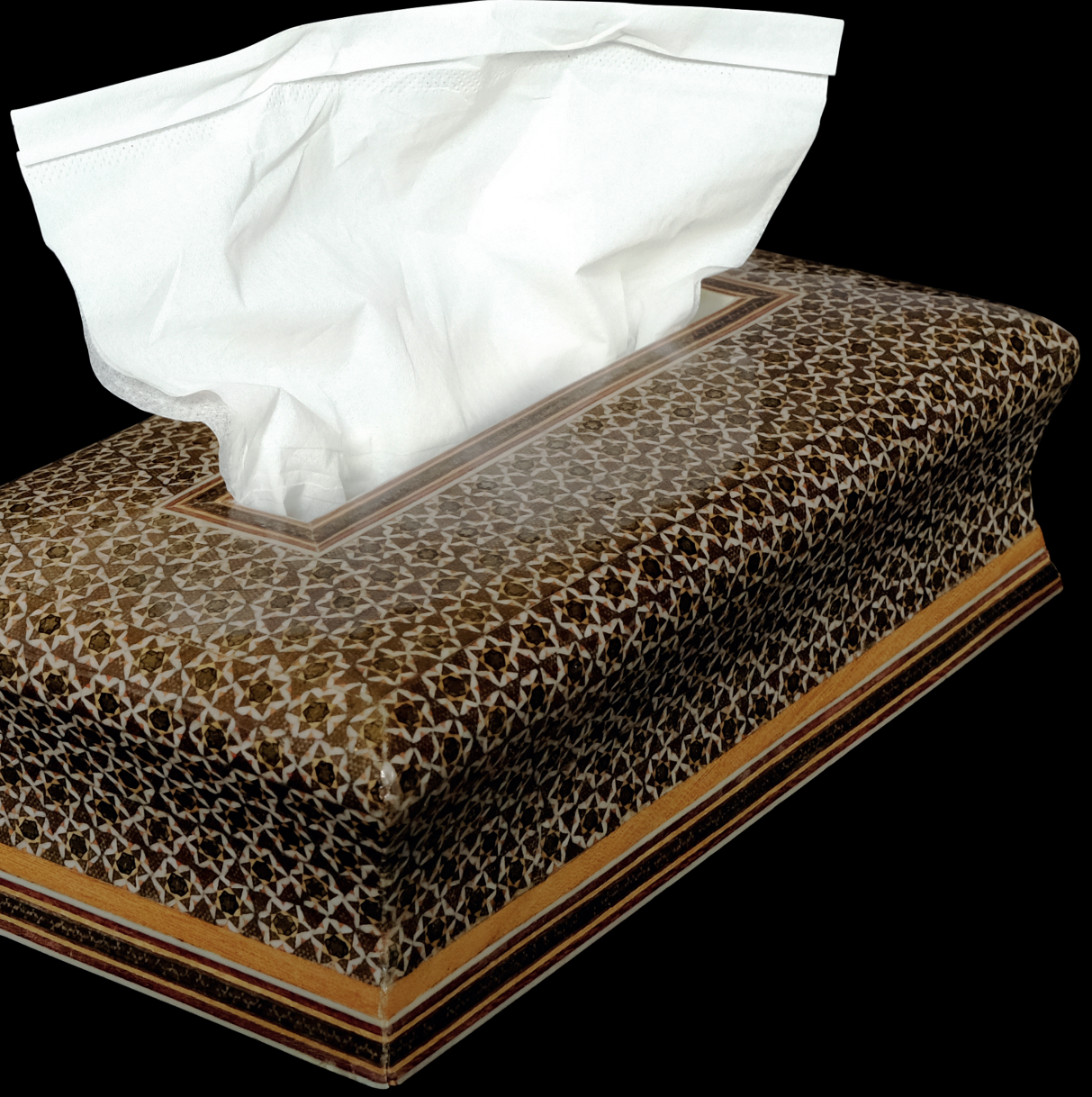


REFERENCES 
Abély, P. (1927). Etat schizophrénique et tendances homosexuelles. Annales-Medicopsychologiques, 2, 251-257.

Abély, P. (1930). Le signe du miroir dans les psychoses et plus spécialement dans la démence précoce. Annales Médico-Psychologiques, 88, 28-36.

Al-Hebshi, N. N., \& Skaug, N. (2005). Khat (catha edulis) - an updated review. Addiction Biology, 10, 299-307. https://doi.org/10.1080/13556210500353020

Al-Motarrieb, A., Al-Habori, M., \& Broadley, K. J. (2010). Khat chewing, cardiovascular diseases and other internal medical problems: the current situation and directions for future research. Journal of Ethnopharmacology 132, 540-48. https://doi.org/10.1016/i. jep.2010.07.001

Anderson, J. R. (1984). The development of self-recognition: A review. Developmental Psychobiology, 17, 35-49.

American Psychiatric Association (2000). Diagnostic and statistical manual of mental disorders (4th ed., text revised). https://doi.org/10.1176/appi.books.9780890423349

American Psychiatric Association (2010). Practice guidelines for treatment of patients with major depressive disorder (3rd ed., pp. 18-19). https://psychiatryonline.org/pb/ assets/raw/sitewide/practice guidelines/guidelines/mdd.pdf

American Psychiatric Association (2013). Diagnostic and statistical manual of mental disorders (5th ed.). https://doi.org/10.1176/appi.books.9780890425596

Ardila, A. (2005). Cultural values underlying psychometric cognitive testing. Neuropsychology Review, 15, 185-195. https://doi.org/10.1007/s11065-005-9180-y

Artiola i Fortuny, L., \& Mullaney, H. A. (1998). Assessing patients whose language you do not know: Can the absurd be ethical? The Clinical Neuropsychologist, 12, 113-126. https:// doi.org/10.1076/clin.12.1.113.1727

Barnett, S. M., \& Williams, W. (2004). National intelligence and the emperor's new clothes. Contemporary Psychology, 49, 389-396. https://doi.org/10.1037/004367

Beach, S. R., Taylor, J. B., \& Kontos, N. (2017). Teaching psychiatric trainees to "think dirty": uncovering hidden motivations and deception. Psychosomatics, 58, 474-482. https:// doi.org/10.1016/j.psym.2017.04.005

Bender, S. D., \& Frederick, R. (2018). Neuropsychological models of feigned cognitive deficits. In: R. Rogers \& S. D. Bender (Eds.). Clinical assessment of malingering and deception (4th ed., pp. 42-60). The Guilford Press.

Benuto, L. T., Leany, B. D., \& Lee, A. (2014). Assessing effort and malingering with the African 
American client. In L.T. Benuto \& B.D. Leany (Eds.). Guide to Psychological Assessment with African Americans (pp. 79-85). Springer.

Bernstein, E. M., \& Putnam, F. W. (1986). Development, reliability, and validity of a dissociation scale. Journal of Nervous and Mental Disease, 174(12), 727-735. https://doi. org/10.1097/00005053-198612000-00004

Berry, D. T., \& Nelson, N. W. (2010). DSM-5 and malingering: A modest proposal. Psychological Injury and Law, 3, 295-303. https://doi.org/10.1007/s12207-010-9087-7

Berthelson, L., Mulchan, S. S., Odland, A. P., Miller, L. J., \& Mittenberg, W. (2013). False positive diagnosis of malingering due to the use of multiple effort tests. Brain Injury, 27, 909916. https://doi.org/10.3109/02699052.2013.793400

Bhandari, S. S., \& Dutta, S. (2018). A brief overview of ethnopsychopharmacology. In: N. K. Kalita \& S. Kalita (Eds.). Psychiatry Update: Etnopsychopharmocology. Bhabani Books. https://www.researchgate.net/publication/327828756 A Brief Overview of Ethnopsychopharmacology

Bianchini, K. J., Curtis, K. L., \& Greve, K. W. (2006). Compensation and malingering in traumatic brain injury: a dose-response relationship? The Clinical Neuropsychologist, 20, 831847. https://doi.org/10.1080/13854040600875203

Biringer, F., Anderson, J.R., \& Strubel, D. (1988). Self-recognition in senile dementia. Experimental Aging Research, 14, 177-180. https://doi.org/10.1080/03610738808259745

Blackmore, R., Boyle, J. A., Fazel, M., Ranasinha, S., Gray, K. M., Fitzgerald, G., Misso, M.. \& Gibson-Helm, M. (2020). The prevalence of mental illness in refugees and asylum seekers: A systematic review and meta-analysis. PLoS Medicine, 17(9), e1003337. https://doi.org/10.1371/journal.pmed.1003337

Boon, S., \& Draijer, N. (1993). Multiple personality disorder in The Netherlands: a clinical investigation of 71 patients. American Journal of Psychiatry, 150, 489-494. https:// doi.org/10.1176/ajp.150.3.489

Boskovic, I., Van Der Heide, D., Hope, L., Merckelbach, H., \& Jelicic, M. (2017). Plausibility judgments of atypical symptoms across cultures: An explorative study among Western and non-Western experts. Psychological Injury and Law, 10, 274-281. https://doi. org/10.1007/s12207-017-9294-6

Bostwick, J. M., \& Chozinski, J. P. (2002). Temporal competency in catatonia. Journal of the American Academy of Psychiatry and the Law, 30, 371-6.

Borsboom, D. \& Cramer, A. O .J. (2013). Network analysis: An integrative approach to the 
structure of psychopathology. Annual Review of Clinical Psychology, 9, 91-121. https:// doi.org/10.1146/annurev-clinpsy-050212-185608

Bot, H. (2005). Dialogue interpreting in mental health. Rodopi.

Braakman, M. H., Kortman, F. A. M., Koeter, M. W., Verkes R. J., \& Van den Brink, W. (2013). Clinical differences between psychosis in 'posttraumatic stress disorder with secondary psychotic features' and schizophrenia among refugees. In: M. H. Braakman. Posttraumatic stress disorder with secondary psychotic features (pp. 47-67). [Doctoral Dissertation, Radboud University]. Radboud Repository.

Brenneisen, R., Fisch, H. U., Koelbing, U., Geisshüsler, S., \& Kalix, P. (1990). Amfetamine-like effects of the khat alkaloid cathinone. British Journal of Clinical Pharmacology 30, 825-28. https://doi.org/10.1111/i.1365-2125.1990.tb05447.x

Bureau InterCulturele Evaluatie (1998). Handleiding Trajectkeuze-toets. Sanoma.

Bush, S. S., Ruff, R. M., Tröster, A. I., Barth, J. T., Kofflerr, S. P., Pliskin, N. H., Reynolds, C. R., \& Silver, C. H. (2005). Symptom validity assessment: Practice issues and medical necessity: NAN Policy \& Planning Committee. Archives of Clinical Neuropsychology, 20, 419-426. https://doi.org/10.1016/i.acn.2005.02.002

Cafasso, J. (2020, June 18) Average IQ: US, globally, how it's measured, and controversies. Healthline. https://www.healthline.com/health/average-iq

Carlson, E. B., \& Rosser-Hogan, R. (1993). Mental health status of Cambodian refugees ten years after leaving their homes. American Journal of Orthopsychiatry, 63, 223-231. https://doi.org/10.1037/h0079422

Carneiro, D. R., Sousa, M., Morgadinho, A., \& Januário, C. (2019). Dopamine dysregulation syndrome induced by proxy. BMJ Case Reports CP, 12, e228495. https://doi. org/10.1136/bcr-2018-228495

Cirlugea, O. (2014). Assessing effort and malingering with the Asian client. In L.T. Benuto, N.S. Thaler, \& B.D. Leany (Eds.). Guide to psychological assessment with Asians (pp. 165-180). Springer.

Colman, A. M. (2014). A Dictionary of Psychology (3rd. ed.). Oxford University Press. https:// doi.org/10.1093/acref/9780199534067.001.0001

Conroy, M. A., \& Kwartner, P. (2006). Malingering. Applied Psychology in Criminal Justice, 2, 29-51.

Correa, A. A. (2018). Beyond borders, Cultural and transnational perspectives of feigning and other response styles. In: R. Rogers \& S.D. Bender (Eds.). Clinical assessment of 
malingering and deception (4th ed., pp. 61-82). The Guilford Press.

Correll, C. U., Detraux, J., De Lepeleire, J., \& De Hert, M. (2015). Effects of antipsychotics, antidepressants and mood stabilizers on risk for physical diseases in people with schizophrenia, depression and bipolar disorder. World Psychiatry,14, 119-136. https:// doi.org/10.1002/wps.20204

Cox, G., \& Rampes, H. (2003). Adverse effects of khat: a review. Advances in Psychiatric Treatment 9, 456-63. https://doi.org/10.1192/apt.9.6.456

Crivelli, C., Jarillo, S., Russell, J., \& Fernández-Dols, J.-M. (2016). Reading emotions from faces in two indigenous societies. Journal of Experimental Psychology: General, 145, 830843. https://doi.org/10.1037/xge0000172

Dandachi-FitzGerald, B., Merckelbach, H., \& Ponds, R. W. (2017). Neuropsychologists' ability to predict distorted symptom presentation. Journal of Clinical and Experimental Neuropsychology, 39, 257-264. https://doi.org/10.1080/13803395.2016.1223278

Dandachi-FitzGerald, B., Ponds, R. W. H. M., Peters, M. J. V., \& Merckelbach, H. (2011). Cognitive underperformance and symptom over-reporting in a mixed psychiatric sample. Clinical Neuropsychology, 25, 812-828. https://doi.org/10.1080/13854046.2011.583280

Dandachi-FitzGerald, B., Van Twillert, B., Van de Sande, P., Van Os, Y., \& Ponds, R. W. H. M. (2016). Poor symptom and performance validity in regularly referred hospital outpatients: Link with standard clinical measures and role of incentives. Psychiatry Research, 239, 47-53. https://doi.org/10.1016/i.psychres.2016.02.061

Darwin, C. (1872). The expression of the emotions in man and animals. John Murray. https:// doi.org/10.1037/10001-000

Delfour, F., \& Marten, K. (2001). Mirror image processing in three marine mammal species: Killer whales (Orcinus orca), false killer whales (Pseudorca crassidens) and California sea lions (Zalophus californianus). Behavioural Processes, 53, 181-190. https://doi. org/10.1016/s0376-6357(01)00134-6

Delmas, M. (1929). Le signe du miroir dans la démence précoce. Annales Médico-Psychologiques, $87,227-233$.

De Pauw, K., \& Szulecka, T. (1987). Lucid intervals in catatonia: A neuropsychiatric snare for the unwary. British Journal of Psychiatry,151, 561-62. https://doi.org/10.1192/ bjp.151.4.561

Derogatis, L. R., (2001). BSI-18, brief symptom inventory 18; administration, scoring and procedures manual. NCS Assessments. https://doi.org/10.1037/t07502-000 
Di Giacomo, E., \& Clerici, M. (2011). Sexual abuse: An overview on causes and consequences. In: L. E. Hynes (Ed.). Sexual abuse: Types, signs and treatments (pp. 93-109). Nova Science Publishers.

Drob, S. L., Meehan, K. B., \& Waxman, S. E. (2009). Clinical and conceptual problems in the attribution of malingering in forensic evaluations. Journal of the American Academy of Psychiatry and the Law, 37, 98-106.

DuAlba, L., \& Scott, R. L. (1993). Somatization and malingering for workers' compensation applicants: A cross-cultural MMPI study. Journal of Clinical Psychology, 49, 913-917. https://doi.org/10.1002/1097-4679(199311)49:6<913::aid-jclp2270490619>3.0.c0;2-1

Edwards, J., Jackson, H. J., \& Pattison, P. E. (2002). Emotion recognition via facial expression and affective prosody in schizophrenia: A methodological review. Clinical Psychology Review, 22, 789-832. https://doi.org/10.1016/s0272-7358(02)00130-7

Egeland, J., Andersson, S., Sundseth, $\varnothing . \varnothing$., \& Schanke, A. K. (2015). Types or modes of malingering? A confirmatory factor analysis of performance and symptom validity tests. Applied Neuropsychology: Adult, 22, 215-226. https://doi.org/10.1080/2327 $\underline{9095.2014 .910212}$

Ekman, P. (2016). What scientists who study emotion agree about. Perspectives on Psychological Science, 11, 31-34. https://doi.org/ 10.1177/1745691615596992

Ekman, P., \& Friesen, W. V. (1971). Constants across cultures in the face and emotion. Journal of Personality and Social Psychology, 17, 124-129. https://doi.org/10.1037/h0030377

Erdodi, L. A., Nussbaum, S., Sagar, S., Abeare, C. A., \& Schwartz, E. S. (2017). Limited English proficiency increases failure rates on performance validity tests with high verbal mediation. Psychological Injury and Law, 10, 96-103. https://doi.org/10.1007/s12207$\underline{017-9282-x}$

European Council on Refugees and Exiles (2012). Asylum Information Database. http://www. asylumineurope.org

European Council on Refugees and Exiles (2020, April 10). Country Report Netherlands (pp. 47-49). https://www.asylumineurope.org/sites/default/files/report-download/aida nl_2019update.pdf

Fan, Q., Yu, J., Ross, C.A., Keyes B.B., Dai, Y., Zhang, T., Wang, L., \& Xiao, Z., (2011). Teaching Chinese psychiatrists to make reliable dissociative disorder diagnoses. Transcultural Psychiatry, 48, 473-483. https://doi.org/10.1177/1363461511409484

Favaro, A., Maiorani, M., Colombo, G., \& Santonastaso , P. (1999). Traumatic experiences, 
posttraumatic stress disorder, and dissociative symptoms in a group of refugees form former Yugoslavia. Journal of Nervous and Mental Disease, 187, 306-308. https://doi. org/10.1097/00005053-199905000-00007

Feldman, M. D. (2004). Disease or deception? An overview of the issues. In: M. D. Feldman, Playing sick? (pp. 18-32). Routledge

Feldman, M. D., \& Yates, G. P. (2018). Dying to be ill. True stories about medical deception. Routledge. https://doi.org/10.4324/9781315160818

Felner, A. E., \& Waldmeier, P. C. (1979). Cumulative effects of irreversible MAO inhibitors in vivo. Biochemical Pharmacology 28, 995-1002. https://doi.org/10.1016/0006$\underline{2952(79) 90293-4}$

Flockhart, D. A. (2012). Dietary restrictions and drug interactions with monoamine oxidase inhibitors: an update. Journal of Clinical Psychiatry, 73 Suppl 1, 17-24. https://doi. org/10.4088/icp.11096su1c.03

Foote, B., Smolin, Y., Kaplan, M., Legatt, M. E., \& Lipschitz, D. (2006). Prevalence of dissociative disorders in psychiatric outpatients. American Journal of Psychiatry, 163, 623-629. https://doi.org/10.1176/aip.2006.163.4.623

Fox, D. D. (2011). Symptom validity test failure indicates invalidity of neuropsychological tests. The Clinical Neuropsychologist, 25, 488-495. https://doi.org/10.1080/13854046.20 11.554443

Friedl, M. C., Draijer, N., \& de Jonge, P. (2000). Prevalence of dissociative disorders in psychiatric in-patients: the impact of study characteristics. Acta Psychiatrica Scandinavica, 102, 423-428. https://doi.org/10.1034/j.1600-0447.2000.102006423.x

Friedman, M. J., Resick, P. A., Bryant, R. A., Strain, J., Horowitz, M., \& Spiegel, D. (2011). Classification of trauma and stressor-related disorders in DSM-5. Depression and Anxiety, 28, 737-749. https://doi.org/10.1002/da.20845

Frueh, B. C., Elhai, J. D., Grubaugh, A. L., Monnier, J., Kashdan, T. B., Sauvageot J. A., Hamner, M. B., Burkett, B. G., \& Arana, G. W. (2005). Documented combat exposure of US veterans seeking treatment for combat-related post-traumatic stress disorder. British Journal of Psychiatry, 186, 467-72. https://doi.org/10.1192/bjp.186.6.467

Frueh, B. C., Knapp, R. G., Cusack, K. J., Grubaugh, A. L., Sauvageot, J. A., Cousins, V.C., Yim, E., Robins, C. S., Monnier, J., \& Hiers, T. G. (2005). Patients' reports of traumatic or harmful experiences within the psychiatric setting. Psychiatric Services 56, 1123-1133. https://doi.org/10.1176/appi.ps.56.9.1123 
Galant, J. S. (1935). Uber selten vorkommende phänomene bei dementia-praecox-kranken; 17: Uber das spiegelzeichen. Psychiatrisch-Neurologische Wochenschrift, 37, 211-212. Gallup, G. G., Jr., Anderson, J.R., \& Shillito, D. (2002). The mirror test. In M. Bekoff, C. Allen (Eds.), The cognitive animal: Empirical and theoretical perspectives on animal cognition (pp. 325-333). MIT Press.

Gara, M. A., Vega, W. A., Arndt, S., Escamilla, M., Fleck, D. E., Lawson, W. B., Lesser, I., Neighbor, H. W., Wilson, D. R. Arnold, L. M., \& Strakowski, S. M. (2012). Influence of patient race and ethnicity on clinical assessment in patients with affective disorders. Archives of General Psychiatry, 69, 593-600. https://doi.org/10.1001/archgenpsychiatry.2011.2040 Gendron, M., Roberson, D., Van der Vyver, J. M., \& Barrett, L. F. (2014). Perceptions of emotion form facial expressions are not culturally universal: Evidence from a remote culture. Emotion, 14, 251-262. https://doi.org/10.1037/a0036052

Geraerts, E., Merckelbach, H. L. C. J., Jelicic, M. (2007). Het simuleren van posttraumatische stresssymptomen: de Nederlandse versie van de Morel Emotional Numbing Test (MENT). Neuropraxis, 1, 8-12. https://doi.org/10.1007/bf03079115

Geraerts, E., Kozaric-Kovacic, D., Merckelbach, H., Peraica, T., Jelicic, M. \& Candel, I. (2009). Detecting deception of war-related posttraumatic stress disorder. Journal of Forensic Psychiatry and Psychology, 20, 278-285. https://doi.org/10.1080/14789940802340906 Gheorghiev, C. (2011). Le signe du miroir. Approche théorique et expérimentale. Éditions universitaires européennes.

Giesbrecht, T., Lynn, S. J., Lilienfeld, S. O., \& Merckelbach, H. (2008). Cognitive processes in dissociation: An analysis of core theoretical assumptions. Psychological Bulletin, 134, 617-647. https://doi.org/10.1037/0033-2909.134.5.617

Giger, P., Merten, T., Merckelbach, H., \& Oswald, M. (2010). Willentliche Testverfälschung bei Verfahren zur Erfassung von Dissoziation. Praxis der Rechtspsychologie, 20, 131-147.

Gilbertson, A., Torem, M., Cohen, R., Newman, I., Radojicic, C., \& Patel, S. (1992). Susceptibility of common self-report measures of dissociation to malingering. Dissociation: Progress in the Dissociative Disorders, 5, 216-220.

Gillman, K. (2002). MAOI's and CNS stimulants. Https://psychotropical.info/maois-and-cnsstimulants

Giosan, C., Glovsky, V., \& Haslam, N. (2001). The lay concept of 'mental disorder': a cross-cultural study. Transcultural Psychiatry, 38, 317-332. https://doi. org/10.1177/136346150103800303 
Gladwell, M. (2019). Talking to strangers. Penguin.

Goedhart, S., \& Sno, H.N. (2014). Het 'signe du miroir': psychopathologische bespiegelingen aan de hand van een vergeten fenomeen. Tijdschrift voor Psychiatrie, 56, 328-335.

Griffin, G.A., Normington, J., May, R., \& Glassmire, D. (1996). Assessing dissimulation among Social Security disability income claimants. Journal of Consulting and Clinical Psychology, 64, 1425-1430. https://doi.org/10.1037/0022-006x.64.6.1425

Hall, H.V., \& Poirier, J.G. (2011). Detecting malingering and deception: Forensic distortion analysis. CRC Press.

Hausotter, W., \& Schouler-Ocak, M. (2007). Begutachting von Menschen mit Migrationshintergrund und Arbeitnehmern nichtdeutscher Herkunft: Unter medizinischen und psychologischen Aspecten (1. Aufl.). Elsevier, Urban \& Fischer.

Heaton, R. K., Smith, H. H., Lehman, R. A., \& Vogt, A. T. (1978). Prospects for faking believable deficits on neuropsychological testing. Journal of Consulting and Clinical Psychology, 46, 892-900. https://doi.org/10.1037/0022-006x.46.5.892

Heilbronner, R. L., Sweet, J. J., Morgan, J. E., Larrabee, G. J., Millis, S. R., \& Conference Participants 1. (2009). American Academy of Clinical Neuropsychology Consensus Conference Statement on the neuropsychological assessment of effort, response bias, and malingering. The Clinical Neuropsychologist, 23, 1093-1129. https://doi. org/10.1080/13854040903155063

Hirsch, M. S., Walter, R. M., \& Hasterlik, R. J. (1965). Subarachnoid hemorrhage following ephedrine and MAO inhibition. Journal of the American Medical Association, 194, 1259. https://doi.org/10.1001/jama.194.11.1259

Hollander, A. C., Dal, H., Lewis, G., Magnusson, C., Kirkbride, J. B., \& Dalman, C. (2016). Refugee migration and risk of schizophrenia and other non-affective psychoses: cohort study of 1.3 million people in Sweden. The BMJ, 352. https://doi.org/10.1136/bmi.i1030 Horner, M.D., Van Kirk, K.K., Dismuke, C.E., Turner, T.H. \& Muzzy, W. (2014). Inadequate effort on neuropsychological evaluation is associated with increased healthcare utilization. The Clinical Neuropsychologist, 28, 703-713. https://doi.org/10.1080/13854046.20 $\underline{14.925143}$

Houtekamer, C. (2012, July 21). Ziek van Nederland. NRC Weekend. https://www.nrc.nl/ nieuws/2012/07/21/ziek-van-nederland-1127045-a886427

Hunt, E. (2011). Human intelligence. Cambridge University Press.

Hwang, J. P., Yang, C.H., \& Tsai, S.J. (2003). Phantom boarder syndrome in dementia. International 
Journal of Geriatric Psychiatry, 18, 417-420. https://doi.org/10.1002/gps.853 Immigratie \& Naturalisatie Dienst (2016). Protocol Bureau Medische Advisering. https://ind. nl/documents/protocol\%20bma\%202016\%20webversie.pdf

Immigratie \& Naturalisatie Dienst (2020, April 10). Asylum Trends (p. 4). https://ind.nl/en/ Documents/AT_January_2020.pdf Institute of Medicine (2015). Psychological testing in the service of disability determination.

The National Academic Press. https://doi.org/10.17226/21704 Ishida, H. (1954). Le signe du miroir. Folia Psychiatrica et Neurologica Japonica, 8, 1-6. Jacobsen, M., \& Thoresen, S. (2011). The validity of screening for post-traumatic stress disorder and other mental health problems among asylum seekers from different countries. Journal of Refugee Studies, 24, 171-186. https://doi.org/10.1093/irs/feq053

Jelicic, M., Merckelbach, H., \& Bošković, I. (2018). Seven myths about feigning. In H. Otgaar \& M. L. Howe (Eds.), Finding the truth in the courtroom: Dealing with deception, lies, and memories (p. 227-242). Oxford University Press.

Kanaan, R. A. (2018). Conversion disorder and illness deception. In: R. Rogers \& S. D. Bender (Eds.), Clinical assessment of malingering and deception (4th ed., pp. 236-242). The Guilford Press.

Kissane, M., Szymanski, L., Upthegrove, R. \& Katona, C. (2014). Complex posttraumatic stress disorder in traumatized asylum seekers: A pilot study. European Journal of Psychiatry, 28, 137-144. https://doi.org/10.4321/s0213-61632014000300001

Kleijn, W.C., Hovens, J.E.J.M., Rodenburg, J.J., \& Rijnders, R.J.P. (1998). Psychiatrische symptomen bij vluchtelingen aangemeld bij het psychiatrisch centrum de Vonk. Nederlands Tijdschrift voor Geneeskunde, 142, 1724-1728.

Kleijn, W.C., \& Mook, J. (1999). Nederlands-Engelstalige adaptatie van de Harvard Trauma Questionnaire. Centrum'45.

Koninklijke Nederlandse Maatschappij ter bevordering van de Geneeskunde (2014). Kwaliteitsnorm tolkgebruik bij anderstaligen in de zorg. https://www.knmg.nl/adviesrichtlijnen/dossiers/tolkenvergoeding-in-de-zorg.htm.

Kövi, A. (1989). De barakken van Hohensalza. Kok.

Laban, C. J., Gernaat, H. B., Komproe, I. H., Schreuders, B. A., \& De Jong, J. J. T. (2004). Impact of a long asylum procedure on the prevalence of psychiatric disorders in Iraqi asylum seekers in the Netherlands. Journal of Nervous and Mental Disease, 192, 843-851. https://doi.org/10.1097/01.nmd.0000146739.26187.15 
Lacan, J. (1966). Ecrits. Editions du Sueil.

Larrabee, G. J. (2008). Aggregation across multiple indicators improves the detection of malingering: Relationship to likelihood ratios. The Clinical Neuropsychologist, 22, 666-679. https://doi.org/10.1080/13854040701494987

Lim, C. R., Harris, K., Dawson, J.,David J Beard, D. J., Fitzpatrick, R., \& Price, A. J. (2015). Floor and ceiling effects in the OHS: An analysis of the NHS PROMs data set. BMJ Open, 5:e007765. https://doi.org/10.1136/bmjopen-2015-007765

Lippa, S. M. (2017). Performance validity testing in neuropsychology: A clinical guide, critical review, and update on a rapidly evolving literature. The Clinical Neuropsychologist, 32, 391-421. https://doi.org/10.1080/13854046.2017.1406146

Lipsanen, T., Lauerma, H., Peltola, P., \& Kallio, S. (1999). Visual distortions and dissociation. Journal of Nervous and Mental Disease, 187, 109-112. https://doi.org/10.1097/00005053199902000-00007

Loutan, L., Bollini, P., Pampallona, S., Bierens de Haan, D., \& Gariazza, F. (1999). Impact of trauma and torture on asylum seekers. European Journal of Public Health, 9, 93-96. https://doi.org/10.1093/eurpub/9.2.93

Lustig, S. L. (2008). Symptoms of trauma among political asylum applicants: don't be fooled. Hastings International and Comparative Law Review, 31, 725-734.

Lynn, R., \& Meisenberg, G. (2010). National IQs calculated and validated for 108 nations. Intelligence, 38, 353-360. https://doi.org/10.1016/i.intell.2010.04.007

Lynn, R., \& Vanhanen, T. (2002). IQ and the wealth of the nations. Praeger.

Lynn, R., \& Vanhanen, T. (2006). IQ and global inequality. Washington Summit Publishers.

Maier, T., Schmidt, M. \& Mueller, J. (2010). Mental health and health care utilization in adult asylum seekers. Swiss Medical Weekly, 140, 13110. https://doi.org/10.4414/ smw.2010.13110

Marin, S. J., Doyle, K., Chang, A., Concheiro-Guisan, M., Huestis, M. A., \& Johnson-Davis, K. (2016). One hundred false-positive amphetamine specimens characterized by liquid chromatography time-of-flight mass spectrometry. Journal of Analytical Toxicology, 40, 37-42. https://doi.org/10.1093/jat/bkv101

Martin, P. K., Schroeder, R. W., \& Odland, A.P. (2015). Neuropsychologists' validity testing beliefs and practices: A survey of North American professionals. The Clinical Neuropsychologist. 29, 741-776. https://doi.org/10.1080/13854046.2015.1087597

McColl, H., McKenzie, \& K., Bhui, K. (2008). Mental healthcare of asylum-seekers and refugees. 
Advances in Psychiatric Treatment, 14, 452-459. https://doi.org/10.1192/apt. bp.107.005041

McNally, R. J., Robinaugh, D. J., Wu, G. W., Wang, L., Deserno, M. K., \& Borsboom, D. (2015). Mental Disorders as Causal Systems. Clinical Psychological Science, 3, 836-849 https:// doi.org/10.1177/2167702614553230

Mcwhirter, L., Ritchie, C. W., Stone, J., \& Carson, A. (2020). Performance validity test failure in clinical populations- a systematic review. Journal of Neurology, Neurosurgery \& Psychiatry. https://doi.org/10.1136/innp-2020-323776

Meade, A. W., \& Craig, S. B. (2012). Identifying careless responding in survey data. Psychological Methods, 17, 437-455. https://doi.org/10.1037/a0028085

Meffert, S. M., Musalo, K., McNiel, D. E., \& Binder, R. L. (2010). The role of mental health professionals in political asylum processing. Journal of the American Academy of Psychiatry and the Law Online, 38, 479-489.

Meijer, D. \& Noijons, J. (2008). Gemeenschappelijk Europees referentiekader voor moderne vreemde talen: leren, onderwijzen, beoordelen. Nederlandse Taalunie.

Merckelbach, H., Boskovic, I., Pesy, D., Dalsklev, M., \& Lynn, S. J. (2017). Symptom overreporting and dissociative experiences: A qualitative review. Consciousness and Cognition, 49, 132-144. https://doi.org/10.1016/i.concog.2017.01.007

Merckelbach, H., Giesbrecht, T., Jelicic, M., \& Smeets, T. (2010). Kretenzers in surveys: het probleem van onzorgvuldige respondenten. Tijdschrift voor Psychiatrie, 52, 663-669.

Merckelbach, H., Giesbrecht, T., Van der Kloet-Van Heugten, D., De Jong, J., Meyer, T \& Rietman, K. (2015). The overlap between dissociative symptoms and symptom overreporting. European Journal of Psychiatry, 29, 165-172. https://doi.org/10.4321/ s0213-61632015000300001

Merckelbach, H., Koeyvoets, N., Cima, M., \& Nijman, H.(2001). De Nederlandse versie van de SIMS. De Psycholoog, 10, 586-591

Merckelbach, H., Prins, C., Boskovic, I., Niesten, E., \& à Campo, J. (2018). Alexithymia as a potemtial source of symptom over-reporting: An exploratory study in forensic patients and non-forensic participants. Scandinavian Journal of Psychology, 59, 192-197. https://doi.org/10.1111/sjop.12427

Merckelbach, H. \& Smith, G.P. (2003). Diagnostic accuracy of the Structured Inventory of Malingered Symptomatology (SIMS) in detecting instructed malingering. Archives of Clinical Neuropsychology, 18, 145-152. https://doi.org/10.1093/arclin/18.2.145 
Merckelbach, H., Langeland, W., De Vries, G., \& Draijer, N. (2014). Symptom overreporting obscures the dose-response relationship between trauma severity and symptoms. Psychiatry Research, 217, 215-219. https://doi.org/10.1016/i.psychres.2014.03.018 Merten, T., \& Merckelbach, H. (2013). Symptom validity testing in somatoform and dissociative disorders: A critical review. Psychological Injury and Law, 6, 122-137. https://doi. org/10.1007/s12207-013-9155-x

Merten, T. (2017). Logical paradoxes and paradoxical constellations in medicolegal assessment. Psychological Injury and Law, 10, 264-273. https://doi.org/10.1007/s12207-017-9297-3 Meijer, D., \& Noijons, J. (2008). Gemeenschappelijk Europees referentiekader voor moderne vreemde talen: leren, onderwijzen, beoordelen. Nederlandse Taalunie.

Meyer, J. F., Faust, K. A., Faust, D., Baker, A. M., \& Cook, N. E. (2013). Careless and random responding on clinical and research measures in the addictions: A concerning problem and investigation of their detection. International Journal of Mental Health and Addiction, 11, 292-306. https://doi.org/10.1007/s11469-012-9410-5

Miller, H. A. (2001). M-FAST: Miller-Forensic assessment of symptoms test professional manual. Psychological Assessment Resources.

Mittenberg, W., Patton, C., Yancock, E. M., \& Condit, D. C. (2002). Base rates of malingering and symptom exaggeration. Journal of Clinical and Experimental Neuropsychology 24, 1094-102. https://doi.org/10.1076/jcen.24.8.1094.8379

Mollica, R. F., Caridad, K. R., \& Massagli, M. P. (2007). Longitudinal study of posttraumatic stress disorder, depression, and changes in traumatic memories over time in Bosnian refugees. Journal of Nervous and Mental Disease, 195, 572-579. https:// doi.org/10.1097/nmd.0b013e318093ed2c

Mollica, R., Caspi-Yavin, Y., Bollini, P., Truong, T., Tor, S., \& Lavelle, J. (1992). The Harvard Trauma Questionnaire. Validating a cross-cultural instrument for measuring torture, trauma and posttraumatic stress disorder in Indochinese refugees. Journal of Nervous and Mental Disease, 180, 111-116. https://doi.org/10.1097/00005053-199202000-00008 Montes, O., \& Guyton, M.R. (2014). Performance of Hispanic inmates on the Spanish Miller Forensic Assessment of Symptoms Test (M-FAST). Law and Human Behavior, 38, 428-438. https://doi.org/10.1037//hb0000074

Morel, K. R. (1998). Development and preliminary validation of a forced-choice test of response bias for posttraumatic stress disorder. Journal of Personality Assessment, 70, 299-314. https://doi.org/10.1207/s15327752ipa7002 8 
Morel, K. R., (2010). Differential diagnosis of malingering versus posttraumatic stress disorder (p. 69). Nova Science Publishers.

Morgan, C. (2007). Psychiatric evaluation of asylum seekers: Is it ethical practice or advocacy? Psychiatry (Edgmont), 4, 26-33.

Mujlli, H. M., Bo, X., \& Zhang, L. (2005). The effect of khat (catha edulis) on acute cerebral infarction. Neurosciences (Riyadh), 10, 219-222.

Neppe, V. M. (1992). Capgras'syndrome of the self. Journal of Neuropsychiatry and Clinical Neuroscience, 4, 352. https://doi.org/10.1176/inp.4.3.352

Niesten, I. J., Nentjes, L., Merckelbach, H., \& Bernstein, D. P. (2015). Antisocial features and "faking bad": A critical note. International Journal of Law and Psychiatry, 41, 34-42. https://doi.org/10.1016/i.ijlp.2015.03.005

Niesten, I. J. M., Merckelbach, H., Dandachi-FitzGerald, B., \& Jelicic, M. (2020). The iatrogenic power of labeling medically unexplained symptoms: A critical review and metaanalysis of "diagnosis threat" in mild head injury. Psychology of Consciousness: Theory, Research, and Practice. https://doi.org/10.1037/cns0000224

Nijdam-Jones A, Rosenfeld B. (2017). Cross-cultural feigning assessment: A systematic review of feigning instruments used with linguistically, ethnically, and culturally diverse samples. Psychological Assessment, 29, 1321-36. https://doi.org/10.1037/pas0000438

Ostancow, P. (1934). Le signe du miroir dans la démence précoce. Annales MédicoPsychologiques, 92, 787-790.

Parnas, J., \& Handest, P. (2003). Phenomenology of anomalous self-experience in early schizophrenia. Comprehensive Psychiatry, 44, 121-134. https://doi.org/10.1053/ comp.2003.50017

Perala, J., Suvisaari, J., Saarni, S. I., Kuoppasalmi, K. Isometsa, E. Pirkola, S., Partonen, T., TuulioHendriksson, A., Hintikka, J., Kieseppa, T., Harkanen, T., Koskinen, S., \& Lonnqvist, J. (2007). Lifetime prevalence of psychotic and bipolar I disorders in a general population. Archives of General Psychiatry, 64, 19-28. https://doi.org/10.1001/archpsyc.64.1.19

Peters, M. J. V., Jelicic, M., Moritz, S., Hausschildt, M., \& Jelinek, L. (2013). Assessing the boundaries of symptom over-reporting using the Structured Inventory of Malingered Symptomatology in a clinical schizophrenia sample: Its relation to symptomatology and neurocognitive dysfunctions. Journal of Experimental Psychopathology, 4, 64-77. https://doi.org/10.5127/jep.023811

Pirandello, L. (1926). Uno, nesuno e centomila. Bemporad. 
Plotnik, J. M., de Waal, F.B.M., \& Reiss, D. (2006). Self-recognition in an Asian elephant. Proceedings of the National Academy of Sciences of the USA, 103, 17053-17057. https://doi.org/10.1073/pnas.0608062103

Povinelli, D. J., Rulf, A., Landau, K., \& Bierschwale, D. (1993). Self-recognition in chimpanzees (Pan troglodytes): Distribution, ontogeny and patterns of emergence. Journal of Comparative Psychology, 107, 347-372. https://doi.org/10.1037/0735-7036.107.4.347 Putnam, F. W., Carlson, E.B., Ross, C. A., \& Anderson, G. (1996). Patterns of dissociation in clinical and nonclinical samples. Journal of Nervous and Mental Disease, 184, 673679. https://doi.org/10.1097/00005053-199611000-00004

Radaelli, D., Benedetti, F., Cavallaro, R., Colombo, C., \& Smeraldi, E. (2013). The reality monitoring deficit as a common neuropsychological correlate of schizophrenic and affective psychosis. Behavioral Sciences, 3, 244-252. https://doi.org/10.3390/bs3020244

Rassin, E., \& Merckelbach, H. (1999). The potential conflict between clinical and judicial decision making heuristics. Behavioral Sciences and the Law,17, 237-248. https://doi. org/10.1002/(sici)1099-0798(199904/06)17:2<237::aid-bs|342>3.0.c0;2-5

Reko, A., Bech, P., Wohlert, C., Noerregaard, C. \& Csillag, C. (2015). Usage of psychiatric emergency services by asylum seekers: Clinical implications based on a descriptive study in Denmark. Nordic Journal of Psychiatry, 69, 587-593. https://doi.org/10.310 9/08039488.2015.1019923

Resnick, P. J., West, S. G., \& Wooley, C. N. (2018). The malingering of posttraumatic disorders. In: R. Rogers \& S.D. Bender (Eds.). Clinical assessment of malingering and deception (4th ed., pp. 188-211). The Guilford Press

Richter, K., Peter, L., Lehfeld, H., Zäske, H., Brar-Reissinger, S., \& Niklewski, G. (2018). Prevalence of psychiatric diagnoses in asylum seekers with follow-up. BMC Psychiatry 18, 206. https://doi.org/10.1186/s12888-018-1783-y

Ricken, R., Ulrich, S., Schlattman, P., \& Adli, M. (2017). Tranylcypromine in mind (Part II): Review of clinical pharmacology and meta-analysis of controlled studies in depression. European Neuropsychopharmacology, 8, 714-731. https://doi.org/10.1016/i. euroneuro.2017.04.003

Rijksoverheid (2020, January 5). Rapport onderzoekscommissie langdurig verblijvende vreemdelingen zonder bestendig verblijfsrecht. https://www.rijksoverheid.nl/ documenten/rapporten/2019/06/04/onderzoekscommissie-langdurig-verblijvendevreemdelingen-zonder-bestendig-verblijfsrecht 
Rindermann, H. (2018). Cognitive capitalism. Cambridge University Press.

Rogers, R. (1990). Models of feigned mental illness. Professional Psychology, 21, 182-188. https://doi.org/10.1037/0735-7028.21.3.182

Rogers R. (2018). An introduction to response styles. In: R. Rogers \& S. D. Bender (Eds.). Clinical assessment of malingering and deception (4th ed., pp. 3-17). The Guilford Press.

Rogers, R. (2018). Detection strategies for malingering and defensiveness. In: R. Rogers \& S. D. Bender (Eds.). Clinical assessment of malingering and deception (4th ed., pp. 1841). The Guilford Press.

Rogers, R., Sewell, K. W. \& Gillard, N. D. (2010). Structured interview of reported symptoms 2nd edition: Professional manual. Psychological Assessment Resources.

Rogers, R., Sewell, K.W., \& Goldstein, A. (1994). Explanatory models of malingering: a prototypical analysis. Law and Human Behavior, 18, 543-552. https://doi.org/10.1007/bf01499173

Rosen, G. M. (2004). Malingering and the PTSD data base. In: G. M. Rosen (Ed.). Posttraumatic stress disorder: Issues and controversies (pp. 85-99). John Wiley \& Sons.

Rosen, G. M. (2006). DSM's cautionary guideline to rule out malingering can protect the PTSD data base. Journal of Anxiety Disorders, 20, 530-535. https://doi.org/10.1016/i. janxdis.2005.03.004

Rosen, G. M., \& Phillips, W. R. (2004). A cautionary lesson from simulated patients. Journal of the American Academy of Psychiatry and the Law Online, 32, 132-133.

Rosen, G. M., \& Taylor, S. (2007). Pseudo-PTSD. Journal of Anxiety Disorders, 21, 201-210. https://doi.org/10.1016/i.janxdis.2006.09.011

Rosenzweig, S., \& Shakow, D. (1937). Mirror behavior in schizophrenic and normal individuals. Journal of Nervous and Mental Disease, 86, 166-174. https://doi. org/10.1097/00005053-193708000-00005

Ross, C. A., Joshi, S., \& Currie, R. (1991). Dissociative experiences in the general population: A factor analysis. Hospital and Community Psychiatry, 42, 297-301. https://doi. org/10.1176/ps.42.3.297

Salazar, X.F., Lu, P.H., Wen, J. \& Boone, K.B. (2007). The use of effort tests in ethnic minorities and in non-English-speaking and English as a second language populations. In K.B. Boone (Ed.), Assessment of feigned cognitive impairment: A neuropsychological perspective (pp. 405-427). Guilford.

Schaefer, J., Giangrande, E., Weinberger, D. R., \& Dickinson, D. (2013). The global cognitive impairment in schizophrenia: Consistent over decades and around the world. 
Schizophrenia Research, 150, 42-50. https://doi.org/10.1016/i.schres.2013.07.009 Schroeder, R. W., \& Marshall, P. S. (2011). Evaluation of the appropriateness of multiple symptom validity indices in psychotic and non-psychotic psychiatric populations. The Clinical Neuropsychologist, 25, 437-453. https://doi.org/10.1080/13854046.2011.556668 Shad, M. U., Tamminga, C. A., Cullum, M., Haas, G. L., \& Keshavan, M. S. (2006). Insight and frontal cortical function in schizophrenia: A review. Schizophrenia Research, 86, 54-70. https://doi.org/10.1016/i.schres.2006.06.006

Shandera, A., Berry, D., Clark, J., Schipper, L., Graue, L., \& Harp, J. (2010). Detection of malingered mental retardation. Psychological Assessment, 22, 50-56. https://doi.org/10.1037/ $\underline{\mathrm{a} 0016585}$

Sherman, E., Slick, D. J., \& Iverson, G. L. (2020). Multidimensional Malingering Criteria for Neuropsychological Assessment: A 20-Year Update of the Malingered Neuropsychological Dysfunction Criteria. Archives of Clinical Neuropsychology, 00, 1-30. https://doi.org/10.1093/arclin/acaa019

Shoeb, M., Weinstein, H., \& Mollica, R. (2007). The Harvard trauma questionnaire: adapting a cross-cultural instrument for measuring torture, trauma and posttraumatic stress disorder in Iraqi refugees. International Journal of Social Psychiatry, 53, 447-463. https://doi.org/10.1177/0020764007078362

Sierra, M., Baker, D., Medford, N., \& David, A.S. (2005). Unpacking the depersonalization syndrome: An exploratory factor analysis on the Cambridge Depersonalization Scale. Psychological Medicine, 10, 1523-1532. https://doi.org/10.1017/s0033291705005325

Sierra, M., \& Berrios, G.E. (2000). The Cambridge Depersonalization Scale: A new instrument for the measurement of depersonalization. Psychiatry Research, 93, 153-164. https:// doi.org/10.1016/s0165-1781(00)00100-1

Siman-Tov, M., Bodas, M., Wang, A., Alkan, M., \& Aldini, B. (2019). Impact of traumatic events incurred by asylum seekers on mental health and utilization of medical services. Scandinavian Journal of Trauma, Resuscitation and Emergency Medicine, 27, 85. https://doi.org/10.1186/s13049-019-0665-8

Simeon, D., Guralnik, O., Gross, S., Stein, D.J., Schmeidler, J., \& Hollander, E. (1998). The detection and measurement of depersonalization disorder. Journal of Nervous and Mental Disease, 186, 536-542. https://doi.org/10.1097/00005053-199809000-00004

Sjöqvist, F. (1965). Psychotropic drugs (2) interaction between monoamine oxidase (MAO) inhibitors and other substances. Proceedings of the Royal Society of Medicine, 58, 
967-78. https://doi.org/10.1177/003591576505811p206

Slick, D. J., Sherman, E. M., Iverson, G. L. (1999). Diagnostic criteria for malingered neurocognitive dysfunction: proposed standards for clinical practice and research. The Clinical Neuropsychologist, 13, 545-61. https://doi.org/10.1076/1385-4046(199911)13:04;1$\mathrm{y} ; \mathrm{ft} 545$

Slick, D. J., \& Sherman, E. M. S. (2012). Differential diagnosis of malingering and related clinical presentations. In: E. M. S. Sherman \& B. L. Brooks (Eds.). Pediatric forensic neuropsychology (pp. 113-135). Oxford University Press.

Sloan, M. A., Kittner, S. J., Rigamonti, D., \& Price, T. R. (1991). Occurrence of stroke associated with use/abuse of drugs. Neurology 41, 1358-1364. https://doi.org/10.1212/ wnl.41.9.1358

Smith, G. P. (2008). Brief screening measures for the detection of feigned psychopathology. In R. Rogers (Ed.), Clinical assessment of malingering and deception (3rd ed., pp. 323-339). The Guilford Press.

Smith, G. P., \& Burger, G. K. (1997). Detection of malingering: validation of the Structured Inventory of Malingered Symptomatology (SIMS). Journal of the American Academy of Psychiatry and the Law, 25, 183-189.

Smith-Apeldoorn, S. Y., Veraart, J. K. E., \& Schoevers, R. A. (2019). Definition and epidemiology of treatment resistance in psychiatry. In: Kim Y (Ed.), Treatment resistance in psychiatry: Risk factors, biology, and management (pp. 3-24). Springer.

Sollman, M. J., \& Berry, D.T.R. (2011). Detection of inadequate effort on neuropsychological testing: A meta-analytic update and extension. Archives of Clinical Neuropsychology, 26, 774-789. https://doi.org/10.1093/arclin/acr066

Söndergaard, H. P., \& Theorell, T. (2004). Alexithymia, emotions and PTSD; findings from a longitudinal study of refugees. Nordic Journal of Psychiatry, 58, 185-191. https://doi. org/10.1080/08039480410006214

Spijker, J., Bockting, C. L. H., Meeuwissen, J. A. C., Van Vliet, I. M., Emmelkamp, P. M. G., Hermens, M. L. M., \& Van Balkom, A. L. J. M. (2013). Multidisciplinaire richtlijn Depressie (Derde revisie). Richtlijn voor de diagnostiek, behandeling en begeleiding van volwassen patiënten met een depressieve stoornis. Trimbos-instituut. https://assets-sites.trimbos.

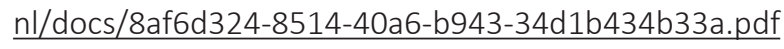

Stein, A. R., Rogers, R., Henry, S. (2018). Denial and misreporting of substance abuse. In: R. Rogers \& S. D. Bender (Eds.). Clinical assessment of malingering and deception (4th 
ed., pp. 151-173). The Guilford Press.

Steinberg, M., \& Schnall, M. (2001). The stranger in the mirror. Quill.

Stevens, A., Schneider, K., Liske, B., Hermle, L., Huber, H., \& Hetzel, G. (2014). Is subnormal cognitive performance in schizophrenia due to lack of effort or to cognitive impairment? German Journal of Psychiatry, 17, 1-9.

Storm, I. (2003). Ervaringen van Marokkaanse en Turkse migranten met een functiebeperking of chronische ziekte in de zorg. http://www.bbvz.nl/files/definitieve rapportage sppmf. pdf

Tirona, R. G., \& Bailey, D. G. (2006). Herbal product-drug interactions mediated by induction. British Journal of Clinical Pharmacology 61, 677-681. https://doi.org/10.1111/i.1365$\underline{2125.2006 .02684 . x}$

Tombaugh, T. N. (1996). TOMM: Test of Memory Malingering. Multi-Health Systems.

Triemstra, M., Veenvliet, C., Zuizewind, C., van Kessel, P., \& Bos, N. (2016). Noodzaak en omvang van de inzet van professionele tolken in de zorg. Nivel.

Tschoner, A., Engl, J., Laimer, M., Kaser, S., Rettenbacher, M., Fleischhacker, W. W., Patsch, J. R., \& Ebenbichler, C. F. (2007). Metabolic side effects of antipsychotic medication. International Journal of Clinical Practice, 61, 1356-1370. https://doi.org/10.1111/ j.1742-1241.2007.01416.x

Ulrich, S., Ricken, R., \& Adli, M. (2017). Tranylcypromine in mind (Part I): Review of pharmacology. European Neuropsychopharmacology 8, 697-713. https://doi.org/10.1016/i. euroneuro.2017.05.007

Van der Heide, D. H. (2007, October). Dissociation induced by self-observation in asylum seekers and refugees with severe mental disorders. Paper presented at the 6th World Congress on Stress.

Van der Heide, D. (2012). In dubio: Why it may not be wise to include asylum seekers in experimental intervention studies (pp. 40-53). ProPersona Mental Health.

Van der Heide, D., Boskovic, I., Van Harten, P, \& Merckelbach, H. (2020). Psychosis as a Confounder of Symptom Credibility Testing in a Transcultural Sample. Journal of Forensic Psychiatry \& Psychology, 31, 64-75. https://doi.org/10.1080/14789949.2 019.1663899

Van der Heide, D., \& Merckelbach, H. (2016). Validity of symptom reports of asylum seekers in a psychiatric hospital: A descriptive study. International Journal of Law and Psychiatry, 
49, 40-46. https://doi.org/10.1016/i.ijlp.2016.05.007

Van der Heide, D., \& Merckelbach, H. (2017). Standard symptom inventories for asylum seekers in a psychiatric hospital: Limited utility due to poor symptom validity. Psychological Injury and Law, 10, 358-367. https://doi.org/10.1007/s12207-017-9302-x

Van Dijk, D. G. L., Kortmann, F., Kooijman, M., \& Bot, J. (1999). De Harvard Trauma Questionnaire (HTQ) als transcultureel screeningsinstrument voor de posttraumatische stress-stoornis bij opgenomen vluchteling. Tijdschrift voor Psychiatrie, 41, 45-49.

Van Dyke, S. A., Millis, S. R., Axelrod, B. N., \& Hanks, R. A. (2013). Assessing effort: Differentiating performance and symptom validity. The Clinical Neuropsychologist, 27, 1234-1246. https://doi.org/10.1080/13854046.2013.835447

Van Impelen, A., Merckelbach, H., Jelicic, M., \& Merten, T. (2014). The Structured Inventory of Malingered Symptomatology (SIMS): a systematic review and meta-analysis. The Clinical Neuropsychologist, 28, 1336-1365. https://doi.org/10.1080/13854046.201 $\underline{4.984763}$

Van IJzendoorn, M. H., \& Schuengel, C. (1996). The measurement of dissociation in normal and clinical populations: Meta-analytic validation of the Dissociative Experiences Scale (DES). Clinical Psychology Review, 16, 365-382. https://doi.org/10.1016/02727358(96)00006-2

Van Veldhuizen, T. (2017). Where I come from and how I got here: Assessing credibility in asylum cases. [Doctoral dissertation. University of Maastricht]. https://doi.org/10.26481/ dis.20170922tsv

Van Wijk, J. (2007). Luanda-Holanda. Irreguliere (asiel)migratie van Angola naar Nederland. [Doctoral dissertation. Radboud University.] Radboud Repository.

Victor, T.L., Boone, K.B., Serpa, J.G., Buehler, J., \& Ziegler, E.A. (2009). Interpreting the meaning of multiple symptom validity test failure. The Clinical Neuropsychologist, 23, 297-313. https://doi.org/10.1080/13854040802232682

Vilar-López, R., Santiago-Ramajo, S., Gómez-Río, M., Verdejo-García, A., Llamas, J.M., \& PérezGarcía, M. (2007). Detection of malingering in a Spanish population using three specific malingering tests. Archives of Clinical Neuropsychology, 22, 379-388. https:// doi.org/10.1016/i.acn.2007.01.012

Wabe, N. T. (2011). Chemistry, pharmacology, and toxicology of khat (catha edulis forsk): a review. Addiction and Health, 3, 137-149.

Waite, S., \& Geddes, A. (2006). Malingered psychosis leading to involuntary psychiatric 
hospitalization. Australasian Psychiatry, 14, 419-421. https://doi.org/10.1080/i.14401665.2006.02317.x

Wallon, H. (1931). Comment se développe chez l'enfant la notion du corps propre. Journal de Psychologie, 705-748.

Weiss, R. A., \& Rosenfeld, B. (2012). Navigating cross-cultural issues in forensic assessment: recommendations for practice. Professional Psychology: Research and Practice, 43, 234-240. https://doi.org/10.1037/a0025850

Weiss, R.A., \& Rosenfeld, B. (2017). Identifying feigning in trauma-exposed African immigrants. Psychological Assessment, 29, 881-889. https://doi.org/10.1037/pas0000381

Werring, D. J. (2010). Reversible cerebral vasoconstriction syndrome. Some answers, many questions. Stroke, 41, 2455-2456. https://doi.org/10.1161/STROKEAHA.110.593905

Wicherts, J. M., Dolan, C. V., \& Van der Maas, H. L. J. (2010). A systematic literature review of the average IQ of sub-Saharan Africans. Intelligence, 38, 1-20. https://doi.org/10.1016/i. intell.2009.05.002

Widows, M. R., \& Smith, G. P. (2005). Structured Inventory of Malingered Symptomatology professional manual. Psychological Assessment Resources.

Wijnkoop, M. (2006). Country assessments: How do EU member states deal with medico-legal reports in asylum procedures? In: A. M. Reneman, R. Bruin \& E. S. A. Bloemen (Eds.). Care Full, Medico-legal reports and the Istanbul Protocol in asylum procedures (pp. 120-209). Pharos/Amnesty International.

Wildman, R. W., \& Wildman, R. W. (1999). The detection of malingering. Psychological Reports, 84, 386-388. https://doi.org/10.2466/pr0.1999.84.2.386

Wisdom, N. M., Pastorek, N. J., Miller, B. I., Booth, J. E., Romesser, J. M., Linck, J. F., \& Sim, A. H. (2014). PTSD and cognitive functioning: Importance of including performance validity testing. The Clinical Neuropsychologist, 28, 128-145. https://doi.org/10.108 $\underline{0 / 13854046.2013 .863977}$

World Health Organization (2001). International classification of functioning, disability and health: ICF. https://apps.who.int/iris/bitstream/handle/10665/42407/9241545429.pdf

World Psychiatric Association Transcultural Psychiatry Section (2020, January 5). Discussion document on refugees - 2017. https://www.wpa-tps.org/wpa-discussion-documenton-refugees-january-2017

Wright, D. B., \& Loftus, E. F. (1999). Measuring dissociation: comparison of alternative forms of the Dissociative Experiences Scale. American Journal of Psychology, 112, 497-519. 
https://doi.org/10.2307/1423648

Yates, G. P., Mulla, M., M., Hamilton, J. C., \& Feldman, M. D. (2018). Factitious disorders in medical and psychiatric practices. In: R. Rogers \& S.D. Bender (Eds.). Clinical assessment of malingering and deception (4th ed., pp. 212-235). The Guilford Press

Yu-Fen, H., \& Neng, T. (1981). Transcultural investigation of recent symptomatology of schizophrenia in China. American Journal of Psychiatry, 138, 1484-1486. https://doi. org/10.1176/aip.138.11.1484

Young, G. (2014). Malingering, feigning, and response bias in psychiatric/psychological injury: implications for practice and court. Springer. https://doi.org/10.1007/978-94-007$\underline{7899-3}$

Young, G. (2014). Slick-Sherman's 2012-2013 revision of the 1999 Slick et al. MND system. In: Young, G. Malingering, feigning and response bias in psychiatric/psychological injury (pp. 385-400). Springer

Young, G. (2015). Malingering in forensic disability-related assessments: Prevalence $15 \pm 15 \%$. Psychological Injury and Law, 8, 188-199. https://doi.org/10.1007/s12207-015-9232-4 Zeck, P. (1961). The dangers of some antidepressant drugs. Medical Journal of Australia, 2, 607-608. https://doi.org/10.5694/i.1326-5377.1961.tb69844.x

Zoroglu, S.S., Sar, V., Tuzun, U., Tutkun, H., \& Savas, H.A. (2002). Reliability and validity of the Turkish version of the adolescent dissociative experiences scale. Psychiatry and Clinical Neurosciences, 56, 551-556. https://doi.org/10.1046/i.1440-1819.2002.01053.x 



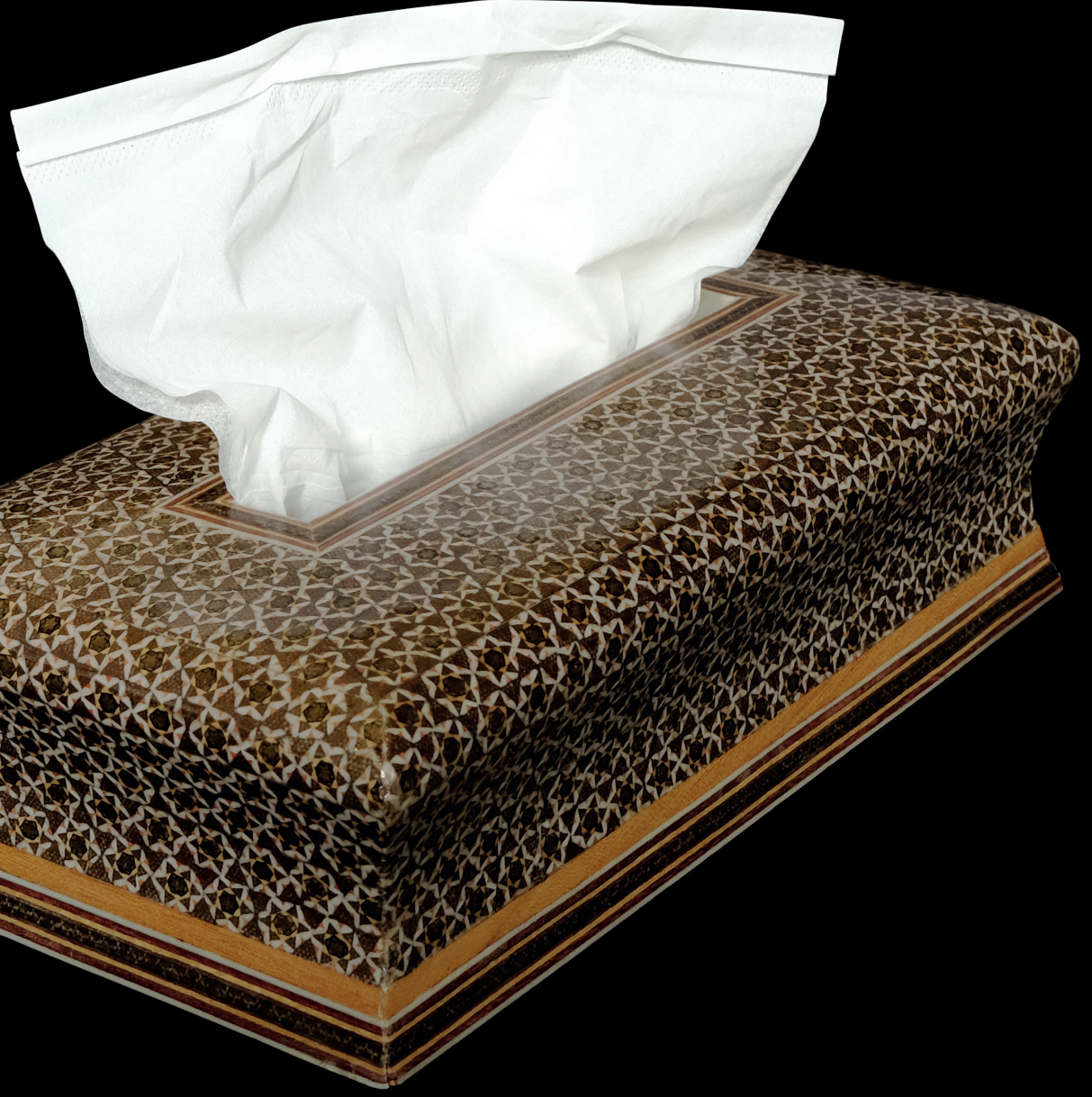


ACKNOWLEDGEMENTS 
The first refugee I got to know was Mr. Kövi, my history teacher in high school. One day he shared a painful episode from his personal history with our class. First he explained that Hungary, his home country, had been an ally of Nazi Germany during World War 2. Then he revealed that he had been drafted into the national army towards the end of the war to help defend Germany. He barely survived the years in Russian captivity that followed ${ }^{1}$.

Mr. Kövi wanted to share the difficult lesson that he had learned: uncritical acceptance of the opinions of your family, church, political party or any other authority can put you on the wrong side of history. I still think about this message today, as I also have a tendency to agree with the opinions of my peers and with authorities. To complete the studies described in this thesis, however, I ultimately had to rely on data rather than on opinions. What followed has considerably affected my perspective on psychiatry.

None of these studies would have been published however, if it were not for Harald Merckelbach, Peter van Harten and Irena Bošković; I am deeply grateful to them for their skillful editing and their unbiased stance towards the topic of this thesis. I also wish to thank all other colleagues who made the effort to assess my findings. This of course includes the members of the PhD examining committee, but also many colleagues in the field of refugee mental health, both in the Netherlands and abroad.

I would like to give a special word of thanks to my former colleagues at Phoenix, the referral center for refugee mental health in Wolfheze where most of the research for this thesis was done. Hanneke Bot, their superintendent, assisted me with her critical comments on each paper before it was submitted. I am also grateful to the hospital management and the Board of Directors of ProPersona Mental Health for allowing me to do this research, and to my present employer, Centraal Mental Health, for the assistance offered to me by its research center Innova.

1) Kövi, A. (1989). De barakken van Hohensalza [The barracks of Hohensalza]. Kok, Kampen. ISBN 90-242-4506-0 
And now I can turn to my family. I suppose you often wondered about this research, what it was that kept me occupied so intensely for so many years. Although at times, I expect, it may also have been a relief to have grumpy old dad locked up in his study.

And finally, a word to my patients. I hope that by now you have found your way into Dutch society and are finally being allowed to enjoy the safety and prosperity that you sought. I also hope that one day you, or perhaps one of your children, will write about your experiences as a refugee in a psychiatric hospital. Perhaps you can do what Kövi finally did, and write a novel about these experiences. You have a painful story to tell, no doubt, but one that still deserves a place in our common history. 


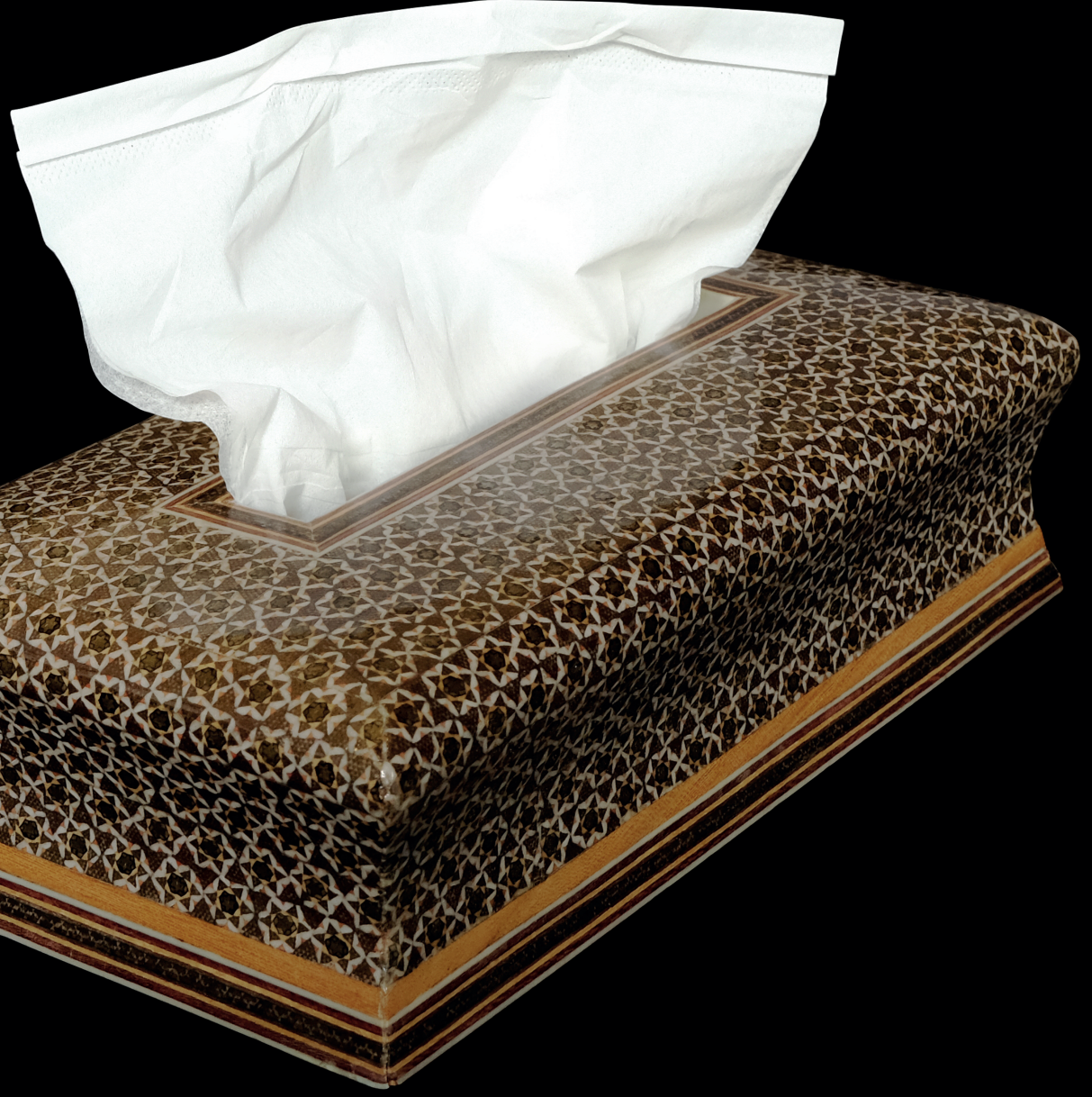


DANKWOORD 
De eerste vluchteling die ik leerde kennen was meneer Kövi, mijn leraar geschiedenis op de middelbare school. Op een dag vertelde hij zijn klas over een pijnlijke periode in zijn eigen geschiedenis. Eerst legde hij uit dat zijn moederland, Hongarije, geallieerd was geweest aan Nazi Duitsland tijdens de oorlog. Daarna vertrouwde hij ons toe dat hij zelf als dienstplichtige in het nationale leger had geholpen bij de verdediging van Duitsland, tegen het eind van de oorlog. Hij had de jaren in Russische krijgsgevangenschap die volgden nauwelijks overleefd ${ }^{1}$.

Zijn les was: het enige dat soms nodig is om aan de verkeerde kant van de geschiedenis te belanden, is zondermeer af te gaan op de mening van je familie, je kerk, politieke partij of welke autoriteit dan ook. Die boodschap raakt me nog steeds, omdat ik me doorgaans uitstekend kan vinden in de opvattingen van mijn vrienden en collega's en die van de autoriteiten. Om de studies die hier zijn beschreven te volbrengen, moest ik uiteindelijk echter vertrouwen op data en niet op meningen. Wat daaruit voortkwam heeft mijn kijk op de psychiatrie aanzienlijk beïnvloed.

Niets van dit alles zou echter zijn gepubliceerd zonder hulp van Harald Merckelbach, Peter van Harten, en Irena Bošković. Ik heb veel te danken aan hun vaardige pen en aan hun onbevooroordeelde houding ten opzichte van het onderwerp van dit proefschrift. Ook alle andere collega's die de moeite namen om mijn bevindingen te bestuderen wil ik bedanken. De leden van de beoordelingscommissie natuurlijk, maar ook al die collega's in de vluchtelingenggz, zowel in Nederland als in het buitenland.

Dat geldt in het bijzonder voor mijn voormalige collega's van Phoenix, het centrum voor vluchtelingen-ggz waar het merendeel van de studies werd uitgevoerd. Hanneke Bot, hun afdelingsbehandelaar, voorzag ieder artikel van het nodige commentaar voordat ik het indiende. Mijn dankbaarheid geldt ook de locatiedirectie en de Raad van Bestuur van ProPersona, die ruimte gaven voor het onderzoek en mijn huidige werkgever GGz Centraal, die me voor het schrijven van dit proefschrift liet aanhaken bij hun onderzoeksinstituut, Innova.

En natuurlijk, mijn gezin. Jullie zullen je wel hebben afgevraagd waar dat onderzoek nu eigenlijk over ging waar ik zo druk mee was. Maar het was misschien ook gewoon wel eens even fijn als jullie kritische oude vader boven op zolder zat te werken.

Kövi, A. (1989). De barakken van Hohensalza. Kok, Kampen. ISBN 90-242-4506-0 
En tot slot, mijn patiënten. Ik hoop van harte dat jullie inmiddels de veiligheid en de voorspoed hebben gevonden die jullie zochten. Maar ook dat iemand van jullie, of wellicht een van jullie kinderen, ooit nog eens opschrijft hoe het is om als vluchteling in een psychiatrisch ziekenhuis opgenomen te zijn. Misschien kunnen jullie doen wat Kövi uiteindelijk gedaan heeft en deze ervaringen verwerken in een roman. Een pijnlijk verhaal, vast en zeker, maar wel een dat een plek verdient in onze gezamenlijke geschiedenis. 


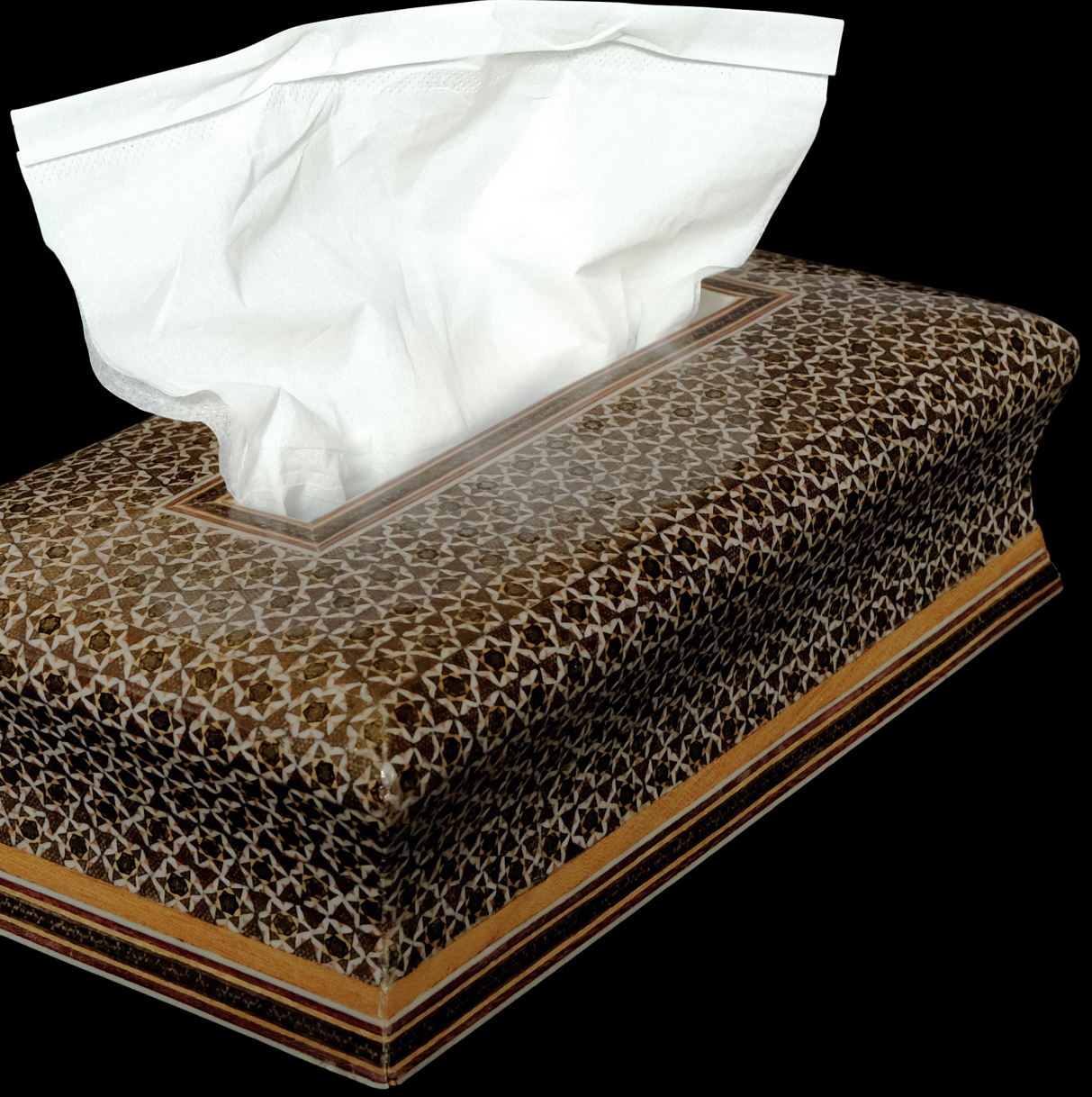


CURRICULUM VITAE 
Douwe van der Heide was born on May $1^{\text {st }} 1961$ in Ferwerderadeel in the Netherlands. He spent his high school years at the Nassau Veluwe College in Harderwijk, and did his general medical training at VU Amsterdam. After working as a doctor in tropical medicine in Zimbabwe, he trained in psychiatry at Veldwijk psychiatric hospital in Ermelo. From 2001 to 2012, he worked as a psychiatrist at Phoenix, a referral center for refugee mental health of ProPersona Mental Health in Wolfheze. Since 2012 he has worked at GGz Centraal Mental Health in Ermelo, currently as regional manager for the Veluwe and Veluwe Valley districts. He holds a master's degree in public health and works as a volunteer for several NGOs in international health and human rights. 
Douwe van der Heide werd geboren op 1 mei 1961 in Ferwerderadeel. Hij bracht zijn middelbare schooltijd door op het Nassau Veluwe College in Harderwijk en studeerde geneeskunde aan de Vrije Universiteit in Amsterdam. Hij werkte als tropenarts in Zimbabwe en deed daarna de opleiding tot psychiater in psychiatrisch ziekenhuis Veldwijk in Ermelo. Van 2001 tot 2012 werkte hij als psychiater in Phoenix, een afdeling voor asielzoekers en vluchtelingen van ProPersona in Wolfheze. Sindsdien werkt hij voor GGz Centraal, op dit moment als directeur behandelzaken voor de Veluwe en de Veluwe Vallei. Hij heeft een master in openbare gezondheidzorg en doet vrijwilligerswerk voor diverse organisaties op het gebied van internationale gezondheidzorg en mensenrechten. 


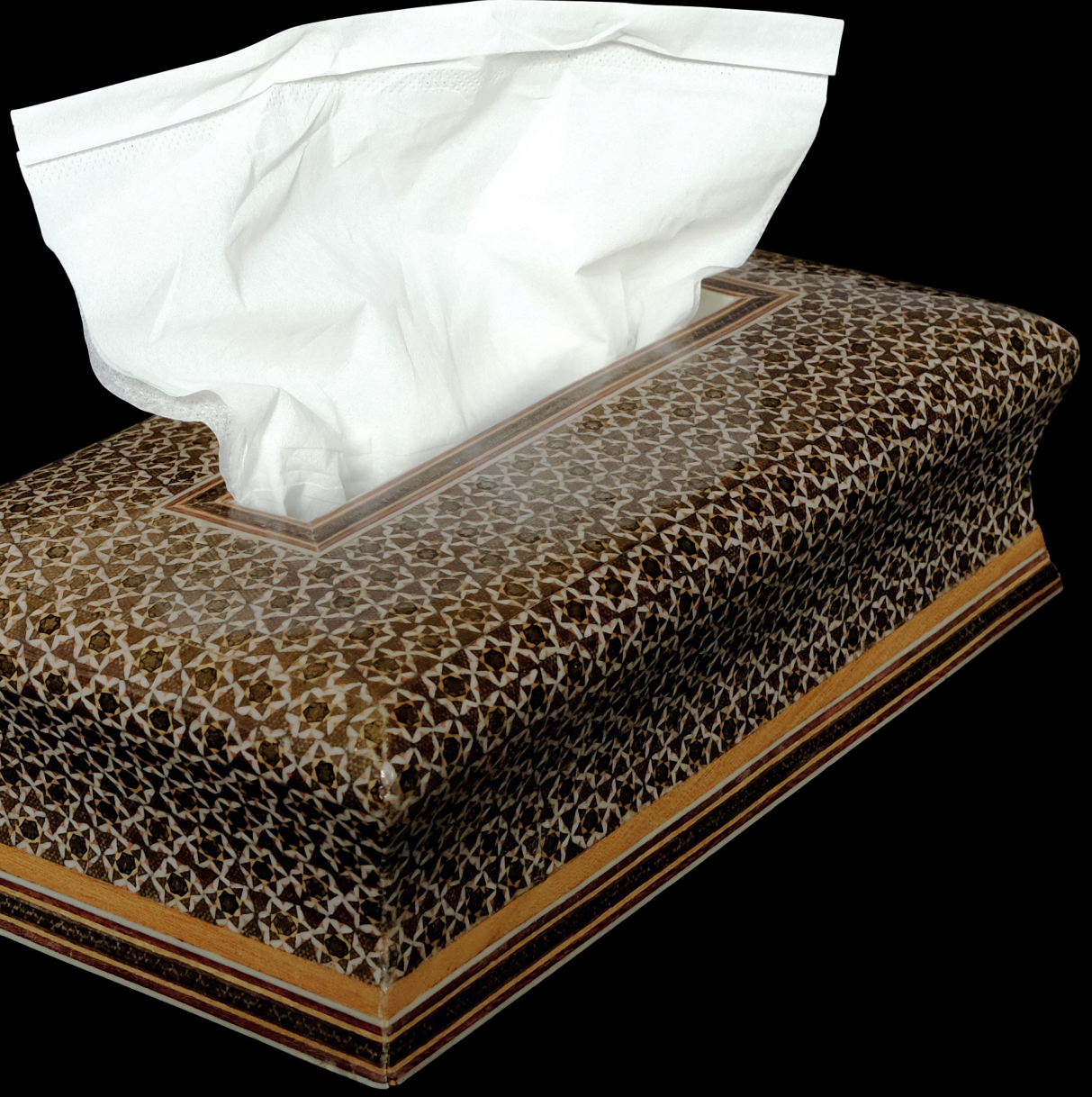




\section{LIST OF PUBLICATIONS}




\section{Articles and chapters}

Van der Heide, D., Boskovic, I., Van Harten, P, \& Merckelbach, H. (2020). Psychosis as a Confounder of Symptom Credibility Testing in a Transcultural Sample. Journal of Forensic Psychiatry \& Psychology, 31, 64-75. https://doi.org/10.1080/14789949.2 $\underline{019.1663899}$

Van der Heide, D., Boskovic, I., Van Harten, P., \& Merckelbach, H. (2020). Overlooking feigning behavior may result in potential harmful treatment interventions: Two case reports of undetected malingering. Journal of Forensic Sciences, 65(4), 1371-1375. https:// doi.org/10.1111/1556-4029.14320

Van der Heide, D., Merckelbach, H., \& Van Harten, P. (2018). Tranylcypromine en khat: Een potentieel fatale combinatie. Tijdschrift voor Psychiatrie, 60(8), 544-547. https:// www.tijdschriftvoorpsychiatrie.nl/assets/articles/60-2018-8-artikel-vanderheide.pdf Van der Heide, D., \& Merckelbach, H. (2017). Standard symptom inventories for asylum seekers in a psychiatric hospital: Limited utility due to poor symptom validity. Psychological Injury and Law, 10, 358-367. https://doi.org/10.1007/s12207-017-9302-x

Boskovic, I., Van Der Heide, D., Hope, L., Merckelbach, H., \& Jelicic, M. (2017). Plausibility judgments of atypical symptoms across cultures: An explorative study among Western and non-Western experts. Psychological Injury and Law, 10, 274-281. https://doi.org/10.1007/s12207-017-9294-6

Van der Heide, D., \& Merckelbach, H. (2016). Validity of symptom reports of asylum seekers in a psychiatric hospital: A descriptive study. International Journal of Law and Psychiatry, 49, 40-46. https://doi.org/10.1016/i.ijlp.2016.05.007

Van der Heide, D., \& Merckelbach, H. (2016). Het spiegelteken: een ernstig symptoom? Nou, nee... Tijdschrift Klinische Psychologie, 2, 121-130.

Bot, H., \& Van der Heide, D. (2010). Duivels dilemma: Waar is Leena beter af: Afrika of Nederland? Psy, 0, 14-17

Bot, H., \& Van der Heide, D. (2010). Drama op uitnodiging. Vluchtelingen met psychische problemen. Maandblad Geestelijke Volksgezondheid, 65, 849-857

Van Bekkum, D. \& Van der Heide, D. (2010). Forensische psychiatrie. In: De Jong \& Colijn (red.). Handboek culturele psychiatrie en psychotherapie (pp. 589-597). De Tijdstroom.

Schouten, K., \& Van der Heide, D. (2009). Traumatic memory in neurobiology and art therapy. In: Hampe, Martius, Ritschl, Von Spreti \& Stalder (Hg.). Kunstreiz. Neurobiologische 
Aspekte Künstlericher Therapien (pp. 525-531). Frank \& Timme.

Van der Heide, D., \& Bos, T. (2005). Samenwerking psychiater en geestelijk verzorger. In: Engelhard \& Goorts (red.). God zal voor mij zorgen. Religieuze coping van vluchtelingen met gezondheidsproblemen (pp. 101-107). Pharos.

Van der Heide, D., \& Braakman, M. (2005). Cultuursensitieve diagnostiek bij somatisatie. In: Eilander, Beers \& De Vos (red.). Verder kijken. Ontwikkelingen in de revalidatiepsychologie (pp. 243-254). Harcourt.

\section{Conference presentations}

Van der Heide, D., Van Harten, P., \& Merckelbach, H. (2021). Why you may not want to include persons seeking asylum for you intervention study. Paper presented at the 13th Annual Research Day of the School for Mental Health and Neuroscience. Maastricht.

Van der Heide, D. (2020). Overlooking feigning. Validity of clinical observation in the detection of feigning. Paper presented at the Zoom - Psychology \& Law Symposium. Online conference, Maastricht University.

Van der Heide, D. (2020 - uitgesteld). Tranylcypromine en khat: Een potentieel fatale combinatie. Lezing op het jaarlijkse congres van de Vereniging Voor Geneeskruiden Onderzoek. Garderen, Nederland.

Van der Heide, D. (2019). Why asylum seekers should not participate in intervention studies. Paper presented at the XXVI $\left.\right|^{\text {th }}$ International Conference on Law and Mental Health, Rome, Italy.

Van der Heide, D. (2019). Are symptom validity tests too difficult for asylum seekers? Paper presented at the $\mathrm{XXV} \mathrm{I}^{\text {th }}$ International Conference on Law and Mental Health, Rome, Italy.

Van der Heide, D. (2019). Are performance validity tests too difficult for asylum seekers? Poster op het Voorjaarscongres van de Nederlandse Vereniging voor Psychiatrie, Maastricht.

Van der Heide, D. (2018). Psychotic symptoms as a confounder of performance validity in asylum seekers. Poster op het Voorjaarscongres van de Nederlandse Vereniging voor Psychiatrie, Maastricht.

Van der Heide, D. (2018). Psychotic symptoms as a confounder of symptom validity in asylum seekers. Poster presented at the World Psychiatric Association Thematic Congress, Melbourne, Australia 
Van der Heide, D. (2017). Validity of symptom reports of asylum seekers in a psychiatric hospital: A descriptive study. Paper presented at the $X X X V^{\text {th }}$ International Conference on Law and Mental Health, Prague, Czech Republic.

Van der Heide, D. (2017). Are symptom validity tests too difficult for asylum seekers? Paper presented at the European Association of Psychology and Law Annual Conference. Mechelen, Belgium.

Van der Heide, D. (2016). Qat (Khat) as a cause of hypertensive crisis in a patient on tranylcypromine. Paper presented at the World Psychiatric Association International Congress, Cape Town, South Africa.

Van der Heide, D. (2015). Cross-cultural assessment of symptom validity. Paper presented at the 4th European Conference on Symptom Validity Assessment, Maastricht, the Netherlands.

Van der Heide, D. (2007). Dissociation induced by self-observation in asylum seekers and refugees with severe mental disorders. Paper presented at the $6^{\text {th }}$ World Congress on Stress, Vienna, Austria.

Van der Heide, D. (2006). Clinical use of the mirror sign. Paper presented at the $9^{\text {th }}$ International Conference on Philosophy, Psychiatry and Psychology, Leiden, the Netherlands.

Visser, H.G., Van der Heide, D.H. (2004). Underestimation of traumatic brain injury in refugees. Paper presented at the International Conference on Differences in Health and in Health Care Provision, Rotterdam, the Netherlands. 